\title{
FOREST AND SCRUB COMMUNITIES WITH GREEN ALDER (ALNUS VIRIDIS) IN SLOVENIA
}

\author{
Igor DAKSKOBLER ${ }^{1,2}$, Andrej ROZMAN ${ }^{2}$ \& Andrej SELIŠKAR ${ }^{3}$
}

\begin{abstract}
This paper provides phytosociological tables that describe scrub and forest communities with Alnus viridis in the Slovenian Alps. We described three new associations: Rhododendro hirsuti-Alnetum viridis (a green alder community on calcareous bedrock in the Eastern and Southeastern Alps), Huperzio selagi-Alnetum viridis (a green alder community in the silicate rocks under Mt. Komen in the eastern Savinja Alps) and Alno viridisSorbetum aucupariae (a successional stage of mountain ash and green alder on potential beech sites in the foothills of the southern Julian Alps; similar stages are known also elsewhere in the Alps), and presented additional three associations (Polysticho lonchitis-Fagetum, Rhodothamno-Laricetum and Rhododendro hirsuti-Pinetum mugo) whose stands comprise green alder.

Key words: phytosociology, synsystematics, Alnetum viridis, Rhododendro hirsuti-Alnetum viridis, Huperzio selagiAlnetum viridis, Alno viridis-Sorbetum aucupariae, the Julian Alps, the Karavanke Mountains, the Smrekovec Mountains.

\section{Izvleček}

V članku s fitocenološkimi tabelami opisujemo grmiščne in gozdne združbe, v katerih v slovenskih Alpah uspeva vrsta Alnus viridis. Opisali smo tri nove asociacije: Rhododendro hirsuti-Alnetum viridis (združba zelene jelše na karbonatni podlagi v vzhodnih in jugovzhodnih Alpah), Huperzio selagi-Alnetum viridis (združba zelene jelše v silikatnem skalovju pod goro Komen v vzhodnih Savinjskih Alpah) ter Alno viridis-Sorbetum aucupariae (sukcesijski stadij jerebike in zelene jelše na potencialno bukovih rastiščih v prigorju južnih Julijskih Alp, podobne stadije poznajo tudi drugod v Alpah) ter predstavili še tri druge asociacije (Polysticho lonchitis-Fagetum, Rhodothamno-Laricetum in Rhododendro hirsuti-Pinetum mugo), v čigar sestojih uspeva zelena jelša.

Ključne besede: fitocenologija, sinsistematika, Alnetum viridis, Rhododendro hirsuti-Alnetum viridis, Huperzio selagi-Alnetum viridis, Alno viridis-Sorbetum aucupariae, Julijske Alpe, Karavanke, Smrekovško pogorje.
\end{abstract}

\section{INTRODUCTION}

Alnus viridis [syn. Alnus alnobetula (Ehrh.) Hartig] is a species with Eurasian and North American distribution. Its nominate subspecies Alnus viridis subsp. viridis is a south-European montane taxon known across the Alpine Arc, in the Pyrenees, Apennines, Carpathians, the Dinaric and
Balkan Mountains (Ball 1993, Aeschimann et al. 2004: 230). It is a character species of the class Betulo carpaticae-Alnetea viridis that incorporates subalpine alder and willow scrub communities. Its communities in the Alps are well studied and are known also in other mountain ranges (e.g. in the Balkan Peninsula - Čolić et al. 1963, Stefanović \& Beus 1982, Stefanović 1986: 211, Tzonev

\footnotetext{
${ }^{1}$ Institute of Biology, Scientific Research Centre of the Slovenian Academy of Sciences and Arts, Regional unit Tolmin, Brunov drevored 13, SI-5220 Tolmin, Igor.Dakskobler@zrc-sazu.si

${ }^{2}$ Biotechnical Faculty of the University in Ljubljana, Department of Forestry and Renewable Forest Resources, Večna pot 83, 1000 Ljubljana, andrej.rozman@bf.uni-lj.si

${ }^{3}$ Grobeljska cesta 6 b, 1234 Mengeš, Slovenia, ase@siol.com
} 
et al. 2009, and in North America - Talbot et al. 2005). A synthetic overview of phytosociological studies of Alnus viridis communities in the Alps was published by Boscutti et al. (2013). In Slovenia, green alder is relatively common only in the Alpine and pre-Alpine phytogeographical regions, but there are individual localities in the $\mathrm{Di}$ naric, pre-Dinaric and sub-Pannonian phytogeographical region (Figure 1). Brus (2008: 33) mentions its occurrence on the Nanos plateau, which would be its southernmost locality in Slovenia. However, the original source of this information is unrealiable and was therefore not included in the distribution map. Green adler sites are mainly in the altimontane and subalpine belt, some also in the lower part of the alpine belt (the highest it was spotted was on Prestreljeniški podi in the Kanin Mountains, at the elevation of around $2250 \mathrm{~m}$ ). Sporadically, it occurs also in the lower montane belt. The lowest locality where it was spotted by the authors was at around $500 \mathrm{~m}$ a.s.l. at Dolenji Novaki in the Cerkljansko region. In literature there are reports of even lower-elevat- ed localities. Most of them are listed by Paulin (1915: 189) for the vicinity of Ljubljana: Šišenski hrib, $400 \mathrm{~m}$ a.s.l.; Velika Trata at Šntvid, $350 \mathrm{~m}$ a.s.l.; Golovec, $400 \mathrm{~m}$ a.s.l.; Hrušica and Bizovik under Golovec, around $350 \mathrm{~m}$ a.s.l.; above the right bank of the Sava at Laze (under Janče), $450 \mathrm{~m}$ a.s.l. (former occurrence of green alder on Rožnik was discussed also by Šercelj, 1996: 66, and by Fleischmann for Golovec, 1844: 136). Very low, at around $400 \mathrm{~m}$ a.s.l., is also the locality on Goričko (Križarka - Boreča) - Bakan (2006: 63). In Slovenia, green alder usually grows on fresh, often acid soil (eutric and dystric brown soils), on mountain hay meadows and pastures, forest edges and reaches its ecological optimum in moist, shady gullies where snowslides occur in the winter. Geological bedrock on its sites is usually mixed (limestone with addition of marlstone, claystone or chert) or silicate (marlstone, claystone, chert, igneous and metamorphic rocks). However, it frequently occurs also on pure limestone, dolomite limestone or dolomite, in places where the soil is moist enough and acid due to

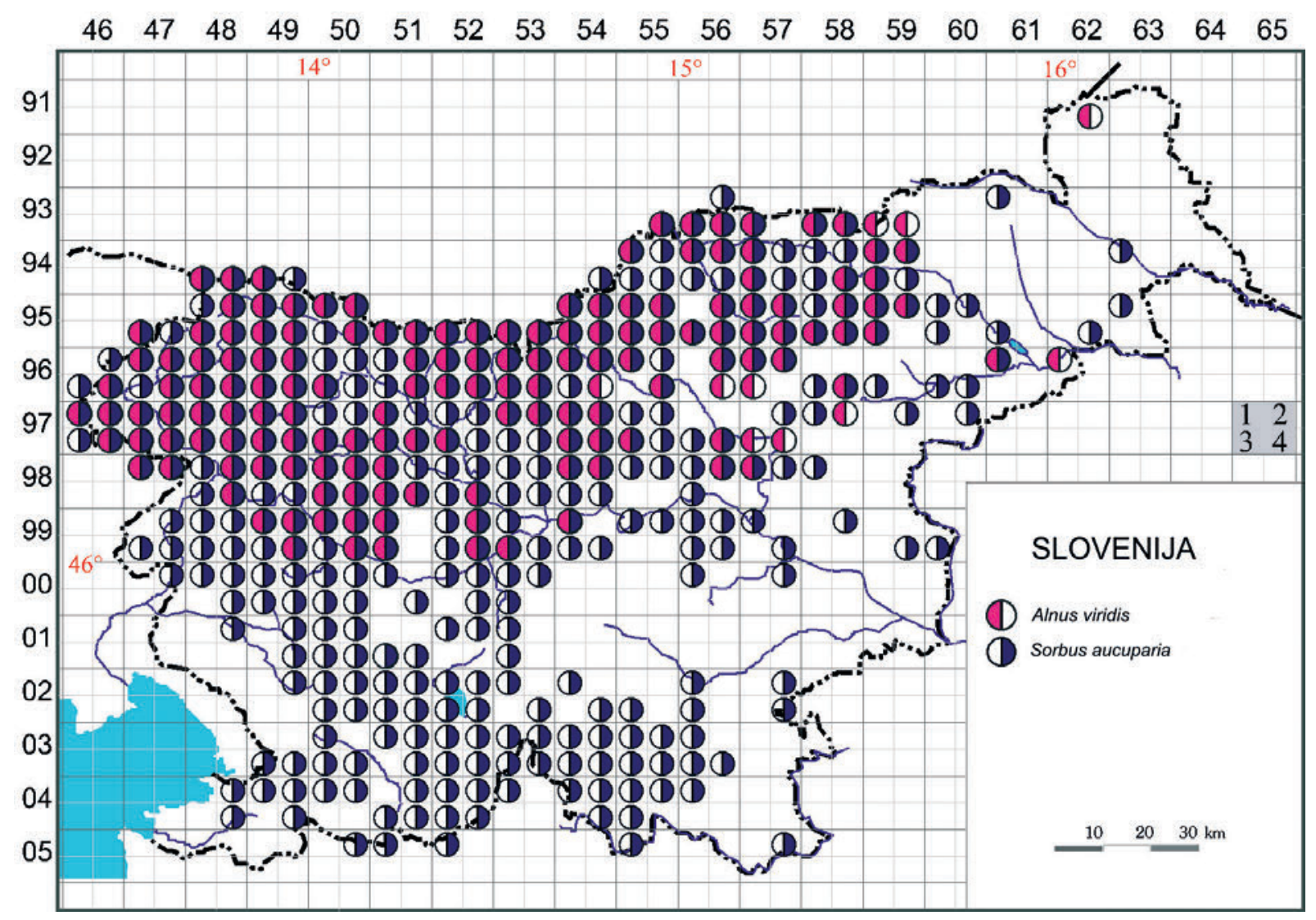

Figure 1: Distribution of Alnus viridis and Sorbus aucuparia in Slovenia Slika 1: Razširjenost vrst Alnus viridis in Sorbus aucuparia v Sloveniji 
slow decomposition of organic matter in surface horizons (moder rendzina). Green alder therefore requires sufficient light and high soil moisture, but has no special temperature requirements and is well-adapted to snow accumulation and resistant to snow mould. Sufficient and constant water supply is crucial for its growth and symbiotic fungi in the soil supply nitrogen from the air (Richard 1969, quoted after Mertz 2000: 374). As a heliophilous species it is not very common in forest communities. It occurs in communities characterised by natural, open tree layer; e.g., very rarely in acidophilous Scots pine stands of the association Vaccinio myrtilli-Pinetum sylvestris (Šercelj 1996: 66), scattered to frequent in eastern-Alpine larch stands (Rhodothamno-Laricetum) - Dakskobler (2006), scattered (constancy 1 or 2) also in several spruce communities (Adenostylo glabrae-Piceetum - Zupančič (1999), Avenello flexuosae-Piceetum - M. Wraber (1955: 15) and Asplenio-Piceetum) and in subalpine beech stands (Polysticho lonchitis-Fagetum). In successional stages it grows also on the sites of the syntaxa LuzuloFagetum abietetosum and Luzulo sylvaticae-Piceetum. It is relatively common in subalpine scrub communities, especially in Alpine dwarf pine scrub (Rhododendro hirsuti-Pinetum mugo) where it has constancy 2 - Zupančič et al. (2006), in the Carphathian birch community (Rhododendro hirsutiBetuletum carpaticae) - Dakskobler et al. (2012) and in subalpine willow communities (Salicetum waldsteinianae - (Zupančič \& Žagar (2001), Surina 2005) and Aceri-Salicetum appendiculatae). One of the first descriptions of green alder stands as a community in the territory of today's Slovenia was published by Paulin (1915: 188-190). When describing the new locality of Athyrium distentifolium $=$ A. alpestre in the Karavanke Mountains he listed all the species growing in the green alder community (formation). The locality of the reléve is under Lepi vrh above the Kočna Saddle in the Struška ridge, at the elevation of around $1680 \mathrm{~m}$. A phytosociological table of green alder stands (Alnetum viridis s. lat.) with four relevés from the Austrian side of the Karavanke was published by Aichinger (1933: 173-174), while Seljak (1974) and Piskernik (1982) published two phytosociological tables of this community in Slovenia. Seljak published six relevés from Mt. Porezen in his graduation thesis and Piskernik applied his own method in making nine relevés on Mt. Porezen and three under Mt. Olševa. According to our opinion Alnus viridis stands occupy a sig- nificant ecological niche and their protective role against erosion and snowslides is important also in the Southeastern Alps but they have not yet been sufficiently phytosociologically researched in Slovenia. By processing the material which we had obtained mainly in the phytosociological research of subalpine beech and larch forests in the Julian Alps, the Kamnik-Savinja Alps and the Karavanke Mountains, we described some of the communities where it occurs.

\section{METHODS}

Vegetation on the sites of Alnus viridis was researched applying the Central-European method (Braun-Blanquet 1964). We made a total of 44 relevés of scrub communities with dominant green alder. These relevés were combined with additional six relevés of this community from Seljak's graduation thesis (1974). These 50 relevés were entered into the FloVegSi database (Seliškar et al. 2003). Combined cover-abundance values were transformed into ordinal values (van der Maarel 1979). Numerical comparisons were made with the software package SYN-TAX (Podani 2001) and R (R Development Core Team 2012), using the package "vegan" (Oksanen et al. 2012). The relevés were compared using the following methods: "(Unweighted) average linkage" - UPGMA, "Incremental sum of squarres" - MISSQ and "Principal Coordinates Analysis" - PCoA. Wishart's similarity ratio was applied in this comparisons. For their suitable syntaxonomic classification a synthetic table was made in which we compared the communities from Slovenia with similar communities from other regions of the Alps in Austria, Italy and Switzerland. Our decision for such comparison was partly based on the fact that we did not have access to all analytic tables, while we did have at our disposal synthetically organised material for the entire territory of Austria. Even more decisive, however, was our conclusion that only the synthetic table provides the means to effectively present the floristic individuality of a syntaxon, which is always an abstract unit and therefore usually requires as many relevés of actual stands in nature as possible for an accurate description. As a rule, none of these stands serves as a typical representative of the described syntaxon, which is only a synthesis of all actual relevés. Although we compared columns, some of which differed 
considerably in the number of relevés, this did not significantly affect the results (e.g. the columns with four and seven relevés did not group together). The columns (syntaxa) in the synthetic table were compared applying the same methods as for the relevés in the analytic table. In order to obtain full information on the sites of green alder in Slovenia we used those relevés from the FloVegSi database that we made of larch, dwarf pine and subalpine beech stands that comprise also this species; these relevés were subsequently arranged into three separate tables (subalpine beech stands, larch stands, dwarf mountain-pine stands). Two pioneer communities with frequent green alder (Alno viridis-Sorbetum aucupariae and Rhododendro hirsuti-Betuletum carpaticae) were also included in the comparison.

A phytoindication analysis in the stands with green alder was carried out using ecological indicator values (Landolt et al. 2010). In the relevés we determined average conditions in terms of temperature $(\mathrm{T})$, continentality $(\mathrm{K})$, light conditions (L), moisture (M), soil reaction (R), nutrients $(\mathrm{N})$, humus content $(\mathrm{H})$ and aeration $(\mathrm{A})$. In our calculations we used van der Maarel's ordinal plant cover values as weight that was reduced by half in indicator values with a higher degree of variation (Landolt et al. 2010).

$W A_{\text {pop }}=\frac{\sum_{i=1}^{s}\left(F V_{i} \times \text { Abund }_{i} \times V R_{i}\right)}{\sum_{i=1}^{s}\left(\text { Abund }_{i} \times V R_{i}\right)}$

where $W A_{p o p}$ is the weighted average of indication values of plant species for an ecological factor on a relevé, $F V_{i}$ is the phytoindication value of the $i$ th species, Abund $_{i}$ is cover value, $V R_{i}$ is range of variation of phytoindication value $(I=1, I I=0.5)$ and $s$ the number of species in a relevé.

Relevés of the stands with green alder were compared using the Canonical Analysis of Principal Coordinates - CAP (Anderson \& Willis 2003) on the basis of Bray-Curtis dissimilarity index where weighted averages of Landolt phytoindication values $\left(W A_{p o p}\right)$ were used as constrained variables.

The nomenclature source for the names of vascular plants is the Mala flora Slovenije (Martinčič et al. 2007). Martinčič $(2003,2011)$ is the nomenclature source for the names of mosses and Suppan et al. (2000) are the nomenclature source for the names of lichenicolous fungi. The nomenclature sources for the names of syntaxa are Theuri- llat (2004) and Šilc \& Čarni (2012). The data on the geological bedrock follow Jurkovšek (1987 a, b) and Buser (2009); the source for the nomenclature of soil types is Urbančič et al. (2005). Ecological description of the Smrekovec Mountains follows Lovrenčak et al. (1998) and Martinčič (2008). Climate data (precipitation volume, mean temperature) were obtained on the website of the Environmental Agency of the Republic of Slovenia, Ministry of Agriculture and Environment (http://www.arso.gov.si/).

\section{RESULTS AND DISCUSSION}

\subsection{Conspectus of Determined AND DESCRIBED SYNTAXA}

Betulo carpaticae-Alnetea viridis Rejmánek in Huml et al. 1979

Alnetalia viridis Rübel ex Huml et al. 1979

Alnion viridis Schnyder 1930

Alnetum viridis Berger 1922 typicum Karner 2007

Rhododendro hirsuti-Alnetum viridis ass. nova hoc loco

Huperzio selagi-Alnetum viridis ass. nova hoc loco

Alno viridis-Sorbetum aucupariae ass. nova hoc loco

Alno viridis-Aceretum pseudoplatani nom. prov.

Querco-Fagetea Br.-Bl. \& Vlieg. 1937

Fagetalia sylvaticae Walas 1933

Aremonio-Fagion (Ht. 1938) Borhidi in Török, Podani \& Borhidi 1989

Polysticho lonchitis-Fagetum (Horvat 1938)

Marinček in Poldini et Nardini 1993

Vaccinio-Piceetea Br.-Bl. in Br.-Bl., Sissingh \& Vlieger 1939 (= Vaccinio-Piceetea Br.-Bl. 1939 emend. Zupančič (1976) 2000)

Piceetalia excelsae Pawłowski in Pawłowski \& al. 1928 (= Vaccino-Piceetalia Br.-Bl. 1939 em. Lund. 1967).

Pinion mugo Pawłowski 1928 (Erico-Pinion mugo Leibundgut 1948)

Rhodothamno-Laricetum (Zukrigl 1973) Willner \& Zukrigl 1999

Alno viridis-Laricetum deciduae nom. prov. Rhododendro hirsuti-Pinetum prostratae Zöttl 1951 (= Rhodothamno-Pinetum mugo Zupančič et Žagar in Zupančič 2013, sensu Zupančič 2013) 


\subsection{Description OF COMMUNities With DOMINANT ALNUS VIRIDIS}

Based on our comparisons (Figure 2) the collected relevés with dominant Alnus viridis in Slovenia were arranged into three columns and compared with green alder communities elsewhere in the Alps. Three types of green alder stands in Austria (Karner 2007a, b) were classified into the synthetic table (Table 4): Alnetum viridis typicum, Alnetum viridis aceretosum pseudoplatani nom. prov. and Alnetum viridis rhododendretosum ferruginei nom. prov., as well as a green alder community (Alnetum viridis s. lat.) on the Austrian side of the Karavanke Mountains (Aichinger 1933), stands of the association Alnetum viridis from Switzerland (Braun-Blanquet 1973) and stands of the association Rhododendro ferruginei-Alnetum viridis from different parts of the Alps (Boscutti et al. 2013). Thus we obtained a table with nine columns which we compared applying hierarchical classification and two-dimensional ordination (PCoA). The results (Figures 3 and 4) demonstrate that the syntaxa form three groups. Among the compared communities the green alder stands recorded in the Smrekovec Mountains in the Savinja Alps stands out the most. The other two of our communities are most similar to the stands of the subassociation Alnetum viridis aceretosum pseudoplatani and to green alder stands from the Austrian side of the Karavanke Mts. The third group comprises the syntaxa Alnetum viridis typicum, Alnetum viridis rhododendretosum ferruginei and Rhododendro ferruginei-Alnetum viridis.

The syntaxa from the first group could be classified into Karner's provisional subassociation Alnetum viridis aceretosum pseudoplatani. In addition to some character species of the association Alnetum viridis s. lat. (e.g. Viola biflora, Saxifraga rotundifolia and Adenostyles alliariae), all differential species of this subassociation are well represented in our stands. However, the dendrogram demonstrates that green alder stands on fresh limestone sites differ substantially from green alder stands on silicate bedrock. Although the analysis conducted by Boscutti et al. (2013) did not confirm this - they determined the occurrence of only two large groups (Alnetum viridis and Rhododendro ferruginei-Alnetum viridis) - our comparison demonstrates that the stands of the subassociation Alnetum viridis aceretosum pseudoplatani are less similar to the stands of the typical form Alnetum viridis typicum than are the stands of the association Rhododendro fer-

MISSQ, similarity ratio

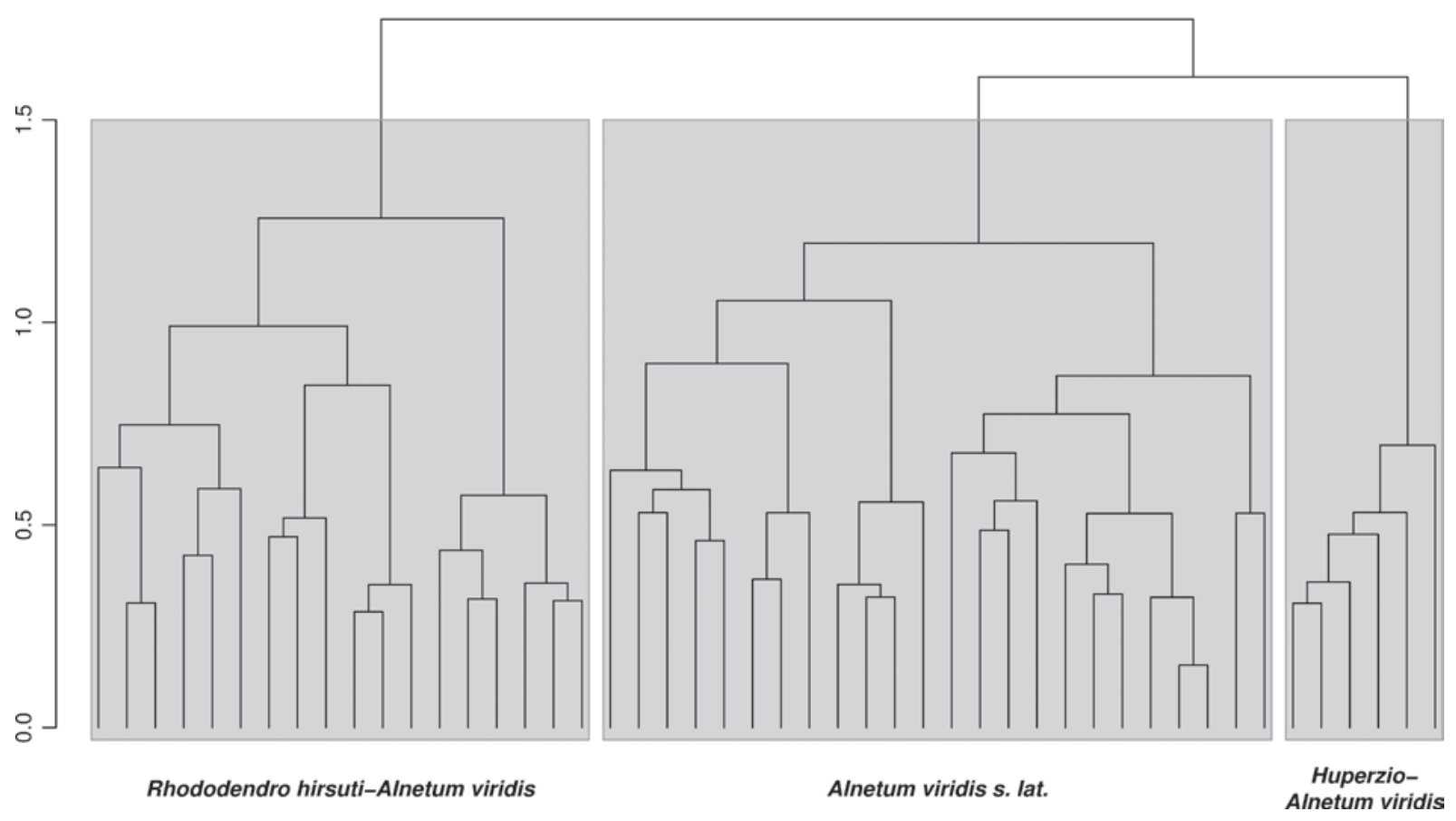

Figure 2: Dendrogram of relevés with dominant Alnus viridis in Slovenia (MISSQ, similarity ratio). Slika 2: Dendrogram popisov z dominantno zeleno jelšo v Sloveniji (MISSQ, similarity ratio). 
UPGMA - Similarity ratio

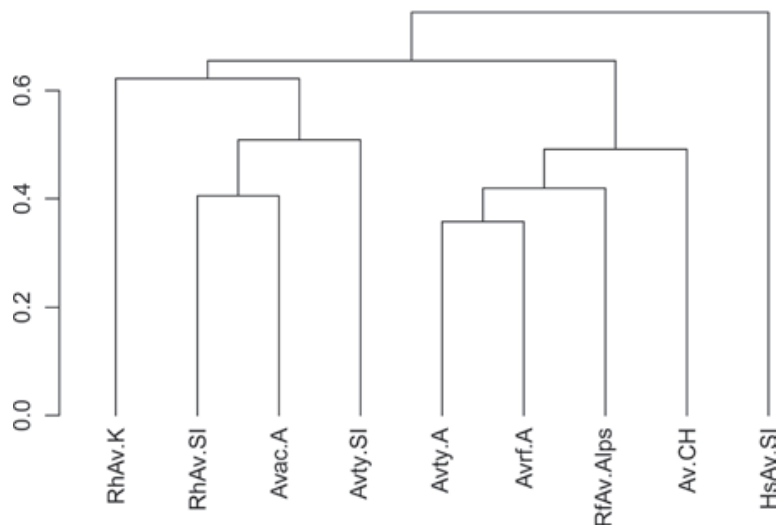

RhAv.SI Rhododendro hirsuti-Alnetum viridis, Slovenia

RhAv.K Rhododendro hirsuti-Alnetum viridis, the Karavanke Mts., Austria

Avty.SI Rhododendro hirsuti-Alnetum viridis / Alnetum viridis, Slovenia

Avac.A Alnetum viridis aceretosum pseudoplatani, Austria

Avty.A Alnetum viridis typicum, Austria

Av. $\mathrm{CH} \quad$ Alnetum viridis, Switzerland

Avrf.A Alnetum viridis rhododendretosum ferruginei, Austria

RfAv.Alps Rhododendro ferruginei-Alnetum viridis, the Alps

HsAv.SI Huperzio selagi-Alnetum viridis, Slovenia

Figure 3: Dendrogram of communities with dominant Alnus viridis in the Alps (UPGMA, similarity ratio).

Slika 3: Dendrogram združb $\mathrm{z}$ dominantno zeleno jelšo $\mathrm{v}$ Alpah (UPGMA, similarity ratio).

ruginei-Alnetum viridis. In our opinion, a higher syntaxonomical rank for green alder stands on silicate bedrock (Alnetum viridis rhododendretosum ferruginei $=$ Rhododendro ferruginei-Alnetum viridis) is justified, and based on our analysis we likewise confirm a higher syntaxonomical rank for green alder stands on calcareous (limestone and dolomite) bedrock. Our comparisons allow the possibility to consider the more or less primary green alder stands on predominantly calcareous bedrock in the Eastern and Southeastern Alps as an independent association Rhododendro hirsuti-Alnetum viridis ass. nova hoc loco and we have therefore increased their rank. Diagnostic (differential) species of the new association are Rhododendron hirsutum, Sorbus chamaemespilus, Polystichum lonchitis, Valeriana tripteris, Asplenium viride, Aconitum lycoctonum s. lat., Galeobdolon flavidum, Thalictrum aquilegiifolium, Salix waldsteiniana, Adenostyles glabra, Cystopteris montana, Acer pseudoplatanus and Primula elatior, which is a differential combination whose frequency ef-
Alnetum viridis - synoptic table

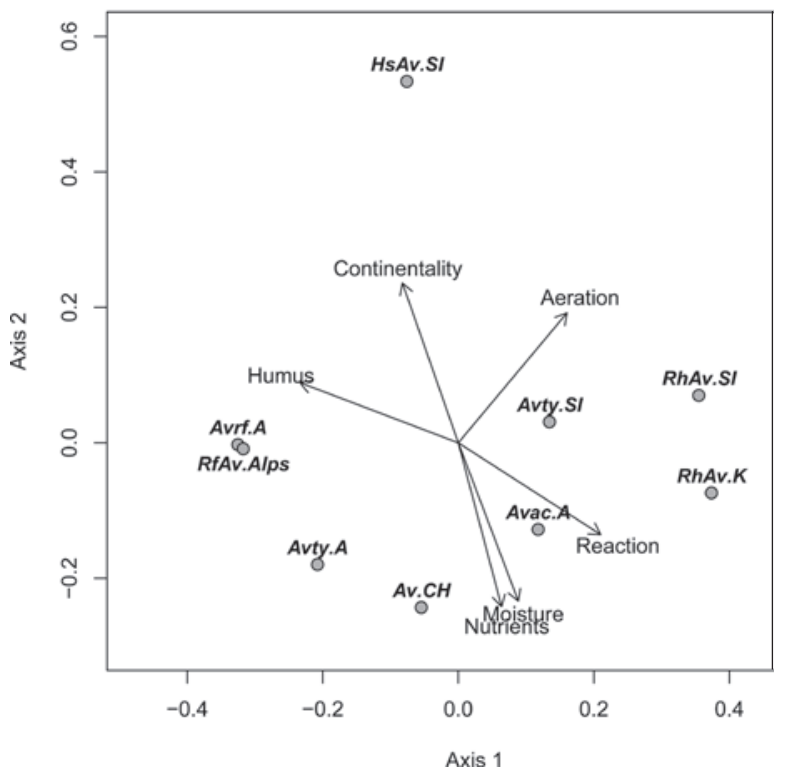

Figure 4: Two-dimensional scatter-diagram of communities with dominant Alnus viridis in the Alps (PCoA, similarity ratio). First two ordination axes explain $34,84 \%$ and $25,31 \%$ of total variation respectively. Arrows represent Landolt's indicator values as passive variables in the analysis.

Slika 4: Dvorazsežni ordinacijski diagram združb z dominantno zeleno jelšo $\mathrm{v}$ Alpah (PCoA, similarity ratio). Prvi dve ordinacijski osi pojasnita $34,84 \%$ oz. $25,31 \%$ skupne variabilnosti. Puščice predstavljajo Landoltove indikacijske vrednosti, kot pasivne spremenljivke $\mathrm{v}$ analizi.

ficiently differentiates these green alder stands from those on silicate bedrock (Alnetum viridis typicum, Rhododendro ferruginei-Alnetum viridis). Some character species of the alliance AremonioFagion (Cardamine enneaphyllos, C. trifolia, Cyclamen purpurascens, Knautia drymeia) that occasionally occur in these stands also have a certain diagnostic (differential) value (see also Table 5, columns 1-4). In central Bosnia, on Mt. Vranica, in the subalpine belt and on silicate and mixed bedrock (Paleozoic slates, phyllite, in places admixture of limestone), Stefanović \& Beus (1982) described the association Athyrio-Alnetum viridis which in places comprises also Rhododendron hirsutum (after which they named the subassociation Athyrio-Alnetum viridis rhododendretosum hirsuti). In some stands of this community there are also other diagnostic species of the association Rhododendro hirsuti-Alnetum viridis: Valeriana tripteris, Polystichum lonchitis, Asplenium viride, Primula elatior, Acer pseudoplatanus and Sorbus chamaemespilus. Nevertheless, the full floristic composition of 
the association Athyrio-Alnetum viridis is considerably different from the composition of the association Rhododendro hirsuti-Alnetum viridis and Sørensen's coefficient of floristic similarity [2c/ $(a+b)$, where $a$ is the number of species in the association Rhododendro hirsuti-Alnetum viridis, $\mathrm{b}$ is the number of species in the association AthyrioAlnetum viridis and $\mathrm{c}$ is the number of species, that are common to both syntaxa] is only about $20 \%-$ Sørensen (1948). The species that differentiate it from the association Rhododendro hirsuti-Alnetum viridis are Athyrium distentifolium (mainly due to its constancy and abundance), Hypericum alpinum (= H. richeri), Laserpitium marginatum $(=$ L. krapfii), Doronicum columnae and Salix silesiaca. The nomenclature type, holotypus, of the new association Rhododendro hirsuti-Alnetum viridis, is relevé No. 5 in Table 1. Relevés from the Southeastern Alps (the Julian Alps, the Karavanke Mountains) are slightly different from the relevés from other parts of the Eastern Alps, so they are treated as a special geographical variant with Homogyne sylvestris. The differential species of this geographical variant are also Festuca nitida, Paederota lutea and Lamium orvala.

Phytosociological Table 1 (see also column 2 in Table 4 and Figure 4), which contains the nomenclature type of the new association, comprises the green alder relevés that were made on steep shady slopes (the usual slope is $30^{\circ}$ to $45^{\circ}$ ), mainly in gullies, on limestone and dolomite bedrock or on their talus slopes. Limestone is very rarely admixed with claystone. The soil is mainly initial, moist, with a more or less thick layer of moder or raw humus, which is the consequence of slow decomposition of organic matter. The soil type is rendzina. The elevation of the relevés is between $1330 \mathrm{~m}$ and $1815 \mathrm{~m}$, the climate is montane, humid, with annual precipitation between $1800 \mathrm{~mm}$ (in the east) and $2500 \mathrm{~mm}$ (in the west) and mean annual temperature between $0{ }^{\circ} \mathrm{C}$ and $4^{\circ} \mathrm{C}$. Snow covers the ground for 150 to 200 days of the year. These relevés were partly made in the forest belt, mainly in the belt of subalpine larch forests from the association RhodothamnoLaricetum, in gullies with snow sliding every year, which prevents normal development of the forest. Green alder stands in these gullies are a long-term successional stage. Some of the relevés were made also above the timberline, at the elevation between $1750 \mathrm{~m}$ and $1815 \mathrm{~m}$, in places where environmental factors still allow scrub growth. In such cases green alder stands represent the final development stage of vegetation and due to their exposed positions progressive development towards subalpine larch or spruce forests is impossible. A relatively rich species composition of these green alder stands is dominated by species of subalpine scrub communities and tall herbs, as well as spruce forest species (see also Table 5, column 2). It comprises also some beech forest species (e.g. Daphne mezereum, Cardamine enneaphyllos, Paris quadrifolia and Mercurialis perennis). There are four distinct variants. The variant typica is represented by only one relevé that was made in Komar above the Zadnjica valley in Trenta, under the rock faces of Kanjavec. Relevés on steep shady slopes under Mt. Črna prst (the highest-lying recorded green alder stands) are classified into the variant with Rhodiola rosea and the green alder stands on talus slopes above the pasture Za Liscem and in the Stara Fužina Pasturelands (under Mizčna glava) into the variant with Soldanella alpina. The variant with Hepatica nobilis (differential species are also Saxifraga cuneifolia, Aconitum tauricum and Helleborus niger) characterises very steep dolomite gullies under Olševa above the Koprivna valley.

Table 2 comprises green alder stands whose common trait is that they usually grow as a pioneer stage on former agricultural land, pastures and hay meadows, still in the belt of beech, firbeech or spruce forest. Geological bedrock is most frequently mixed, limestone or dolomite with addition of marlstone, claystone and chert, or entirely silicate (claystone, igneous rocks). These relevés separated from the relevés of "primary" green alder stands of the association Rhododendro hirsuti-Alnetum viridis (Figure 2), but synthetic comparison (Figures 3 and 4) showed more similarity with them than with green alder stands from other Alpine regions. Phytogeographical factor clearly played a decisive role here. Nevertheless, a major part of these stands is not classified into the new association Rhododendro hirsuti-Alnetum viridis. The only exceptions are relevés 17 to 24 in Table 2 . These were made on northern peak slopes of Mt. Porezen, where the forest had been cleared for pastures a long time ago, and on the foothills of the southern Julian Alps (Kobla, Slatnik, Bizle above Rut). The elevation of these relevés is between $1320 \mathrm{~m}$ and $1600 \mathrm{~m}$ and the ecological conditions (i. e. steep slopes, predominant shady exposition, climate) are very similar to those listed in the description of the new association. Relevés 1 to 5 are 


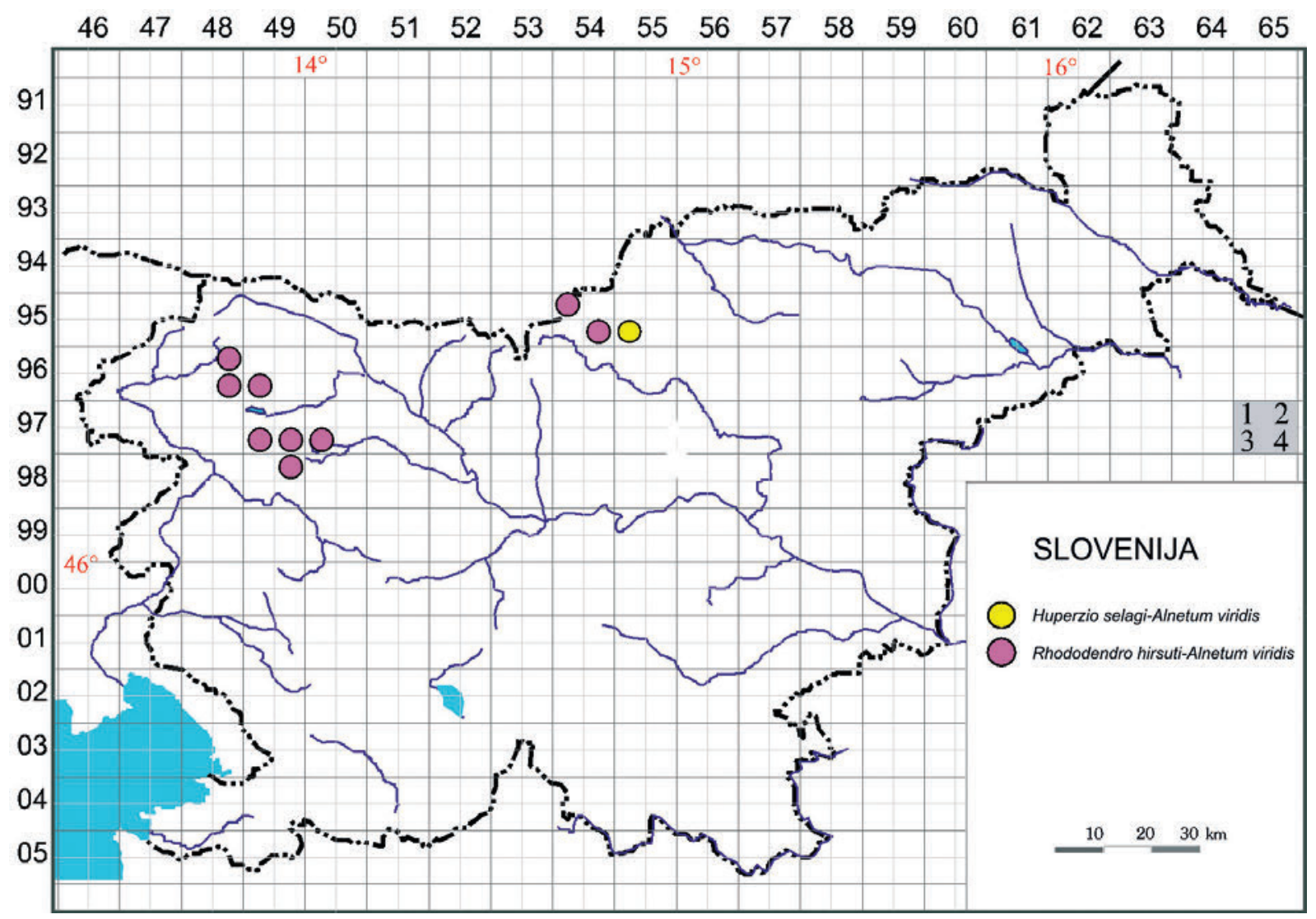

Figure 5: Approximate localities of stands of the associations Rhododendro hirsuti-Alnetum viridis and Huperzio selagi-Alnetum viridis in Slovenia.

Slika 5: Približna nahajališča sestojev asociacij Rhododendro hirsuti-Alnetum viridis in Huperzio selagi-Alnetum viridis $\mathrm{v}$ Sloveniji.

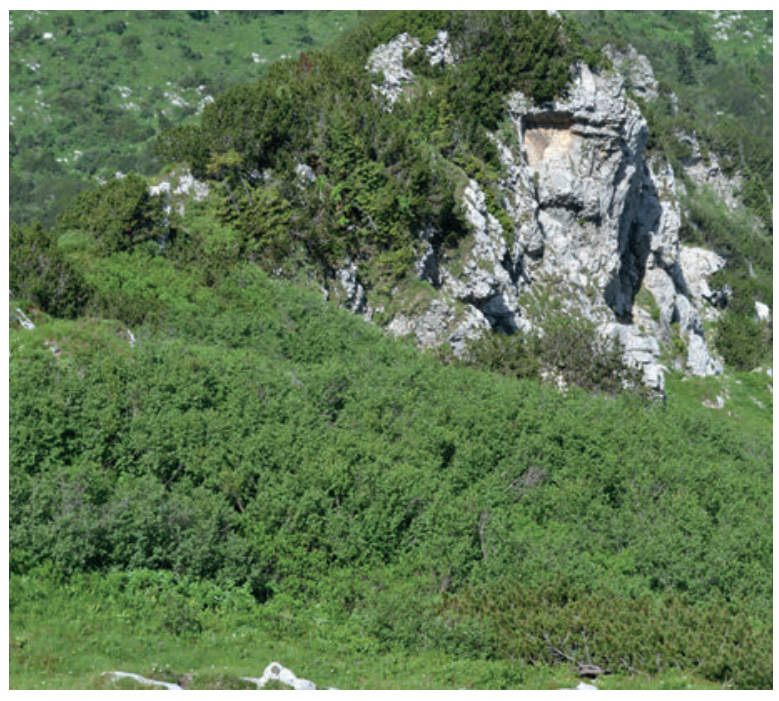

Scrub comunity with dominant Alnus viridis (Rhododendro hirsuti-Alnetum viridis), the northern slopes of Mt. Črna prst. Grmišče s prevladujočo zeleno jelšo (Rhododendro hirsutiAlnetum viridis) na severnih pobočjih Črne prsti. classified into the subassociation Alnetum viridis typicum. These are relevés from the western and eastern Karavanke Mts. and the Smrekovec Mts. They were made at the elevation ranging from $1370 \mathrm{~m}$ to $1720 \mathrm{~m}$ a.s.l., on shady aspects with lower slopes. The climate is montane, but less humid than in the Julian Alps. Character species of the association Alnetum viridis are well represented, which cannot be said for the differential species of the associations Rhododendro hirsuti-Alnetum viridis and Rhododendro ferruginei-Alnetum viridis. Of all the relevés compared, relevés No. 6 to 16 the most clearly stand out as pioneer stands on abandoned alpine pastures on potential beech sites. These relevés were made at the elevation between $1250 \mathrm{~m}$ and $1450 \mathrm{~m}$ on abandoned pastures under Kobilja glava and Jalovnik in the foothills of the Julian Alps; one relevé is from Otavnik near Porezen. These stands are relatively young and character species of the association Alnetum viridis only sporadically occur in their floristic 


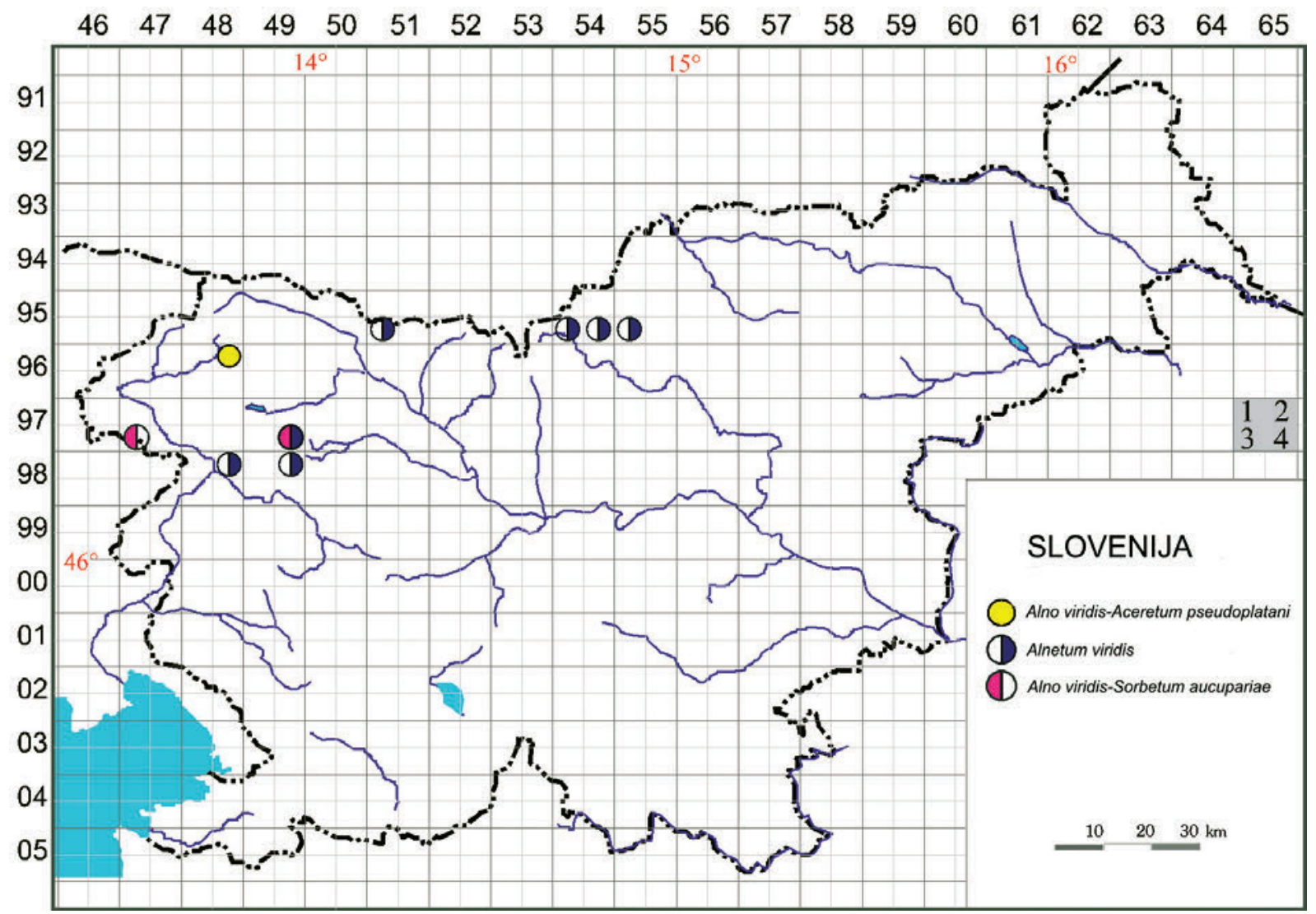

Figure 6: Approximate localities of stands of the associations Alnetum viridis s. lat., Alno viridis-Sorbetum aucupariae and Alno viridis-Aceretum pseudoplatani in Slovenia.

Slika 6: Približna nahajališča sestojev asociacij Alnetum viridis s. lat., Alno viridis-Sorbetum aucupariae and Alno viridis-Aceretum pseudoplatani v Sloveniji.

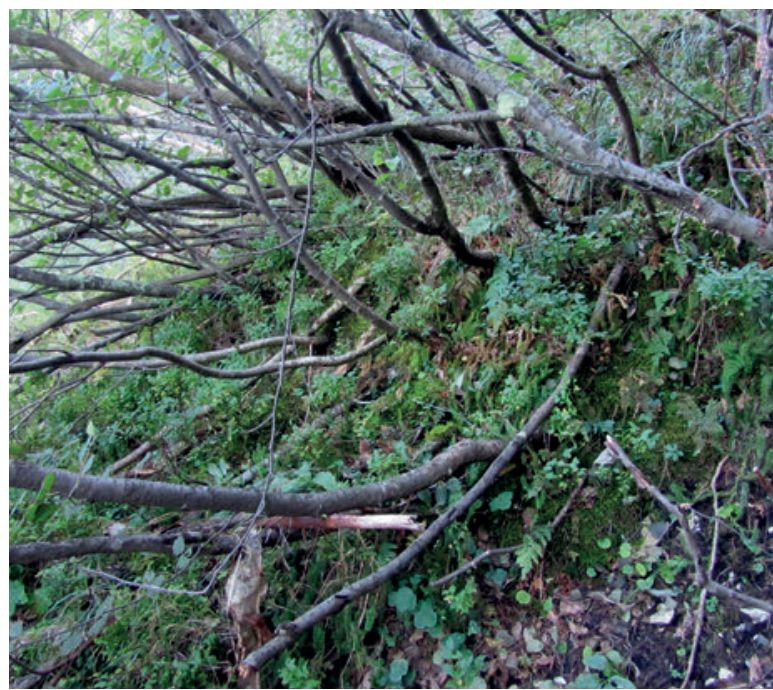

Stand of the association Rhododendro hirsuti-Alnetum viridis on the northern slopes of Mt. Olševa.

Sestoj asociacije Rhododendro hirsuti-Alnetum viridis na severnih pobočjih Olševe. composition. For now, these relevés are treated within the macroassociation Alnetum viridis s. lat., but without a detailed syntaxonomic definition.

Table 3 comprises six relevés that were made in the Smrekovec Mts. in the eastern Savinja Alps, on very steep shady slopes under Mt. Komen (Kamen) at the elevation between $1640 \mathrm{~m}$ and $1670 \mathrm{~m}$ (Figure 5). Geological bedrock is composed of igneous rocks with predominating andesite; the soil type is ranker or shallow dystric Cambisol. The climate is montane, with annual precipitation of around $1800 \mathrm{~mm}$ and mean annual temperature of $0^{\circ} \mathrm{C}$ to $2^{\circ} \mathrm{C}$. These relevés are different from all other relevés compared (compare Figures 2, 3 and 4). Their sites are quite extreme, steep rocks or gullies that cannot be populated by forest vegetation (in this case subalpine spruce forest from the association Luzulo sylvaticae-Piceetum). For now, green alder scrubs therefore remain the highest stage of vegetation development and a long-term successional stage. In gen- 


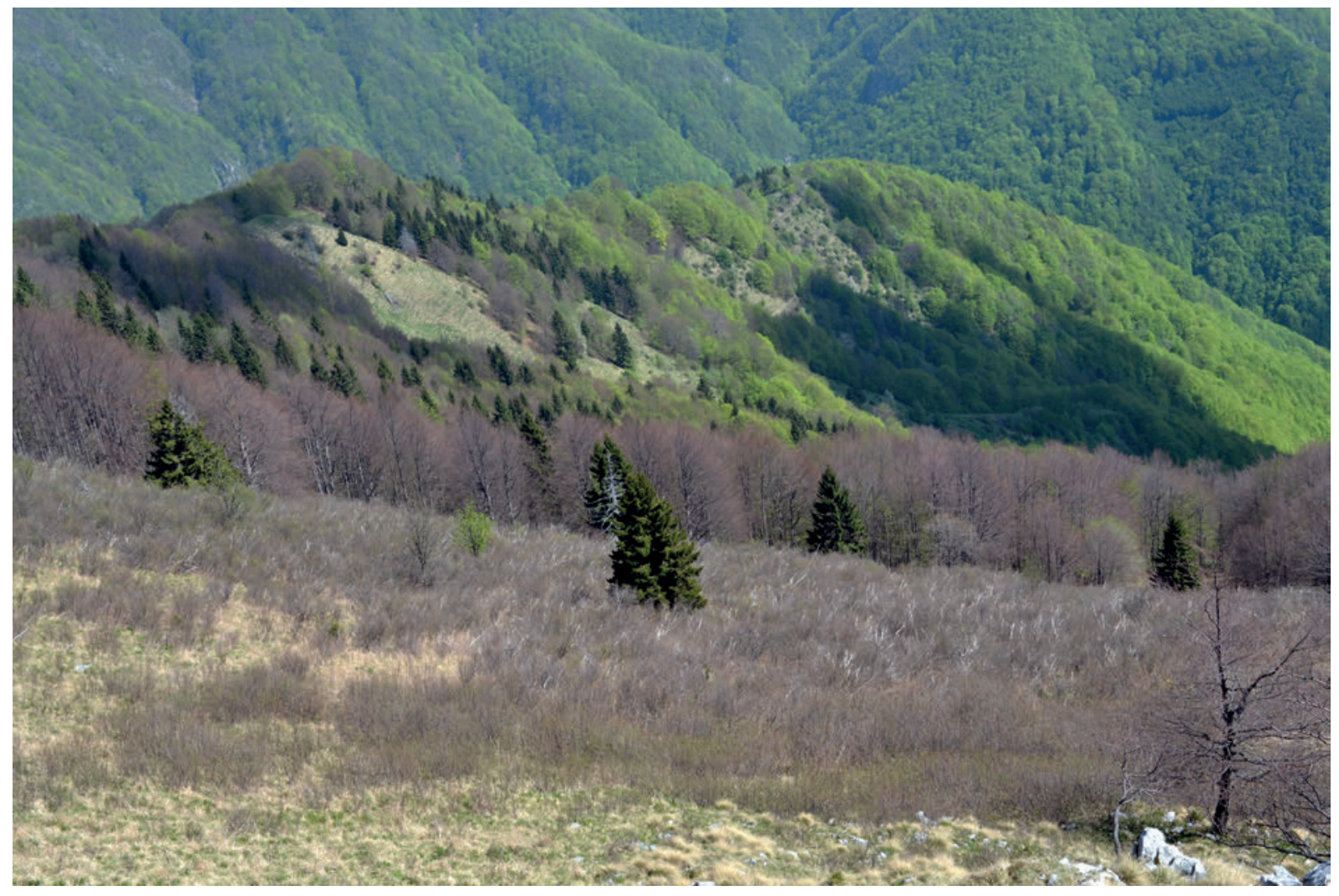

Pioneer green alder scrub community (Alnetum viridis s. lat.) on formerly pastures on potential sites of altimontane beech forests, northern slopes of Mt. Kobilja glava in the southern Julian Alps.

Pionirsko grmišče zelene jelše (Alnetum viridis s. lat.) na nekdanjih pašnikih na potencialnih rastiščih altimontanskega bukovega gozda na severnih pobočjih Kobilje glave v južnih Julijskih Alpah.

eral, fewer species grow in these stands than in the stands of previously described syntaxa. Absent are especially character species of subalpine scrubs and tall herbs from classes Betulo-Alnetea and Mulgedio-Aconitetea, which are also character species of the macroassociation Alnetum viridis s. lat. More common, in addition to the green alder, are sporadically only Viola biflora, Veratrum album subsp. album and Stellaria nemorum. Species with the highest constancy and medium cover belong to the class of spruce forests Vaccinio-Piceetea (see also column 9 in Table 5). They include Vaccinium myrtillus, V. vitis-idaea, Calamagrostis villosa, Picea abies, Avenella flexuosa, Phegopteris connectilis, Dryopteris dilatata, Luzula sylvatica, Lycopodium annotinum, Huperzia selago, Homogyne alpina and Thelypteris limbosperma. Diagnostic value have also some species characteristic for silicate rocks or acid subalpine-alpine grasslands and spring areas, such as Primula villosa, P. minima, Campanula alpina, Agrostis rupestris, Festuca varia and Saxifraga stellaris subsp. prolifera. These stands com- prise also some diagnostic species of the above mentioned or described associations, for example Rhododendron hirsutum, Valeriana tripteris, Vaccinium myrtillus, Rubus idaeus and Dryopteris dilatata; however, these species cannot be classified neither into the association Rhododendro hirsutiAlnetum viridis (due to the absence of most of its diagnostic species and tall herb species), nor into the association Rhododendro ferruginei-Alnetum viridis (into which they should be classified based on the ecological conditions and geological bedrock), because they do not comprise the species Rhododendron ferrugineum nor a number of diagnostic species of the classes Betulo-Alnetea and Mulgedio-Aconitetea. The absence of the latter species does not allow for the classification of these stands into the syntaxon Alnetum virdis typicum. Because of their obvious floristic uniqueness they are classified into the new association Huperzio selagi-Alnetum viridis ass. nova hoc loco. Its nomenclature type, holotypus, is relevé No. 1 in Table 3. Diagnostic species of the new asso- 
ciation are Vaccinium vitis-idaea, Lycopodium annotinum, Huperzia selago, Thelypteris limbosperma, Festuca guestfalica, F. varia, Primula minima, P. villosa and two mosses (the moss layer in this community is in fact relatively rich) - Rhytidiadelphus loreus and Sphagnum russowii. The listed species reflect the characteristics of subalpine sites on silicate rocks with distinctly acid soil. In addition to the typical variant we distinguish also the variant with Vaccinium gaultherioides (the differential species is also Empetrum hermaphroditum) in very steep andesite rocks. The stands of the association Huperzio-Alnetum viridis are yet another vegetation feature of the Smrekovec Mts., which are known for their unique flora and vegetation (Petkovšek 1945, T. Wraber in Lovrenčak et al. 1998, Martinčič 2008, Juvan et al. 2011).

\subsection{DESCRIPTION OF SOME FOREST COMMUNITIES WITH GREEN ALDER IN THE SHRUB LAYER}

\subsubsection{Alno viridis-Sorbetum aucupariae ass. nova}

Table 6 comprises ten relevés of pioneer forest stands whose tree layer is dominated by mountain ash (Sorbus aucuparia) and which developed in the belt of altimontane and subalpine beech forests (Ranunculo platanifolii-Fagetum, Polysticho lonchitis-Fagetum) in the southern Julian Alps and their foothills (Figure 6). Primary forest vegetation has been either cleared for hay meadows or pastures, or destroyed by snowslides (see relevé 4). Green alder was frequently the first to have established itself on steep, often stony or rocky shady slopes after agricultural land use was abandoned, but was replaced in the succession by the mountain ash that now builds the upper stand layer and reaches a tree height of about 10 metres and up to $30 \mathrm{~cm}$ in diameter at breast height. Progressive development into the beech forest is very long on these extreme sites, so it seems appropriate to treat these pioneer stands at the rank of association. Recently, phytosociologists have been incorporating similar pioneer forests on fresh sites in the montane belt whose tree layer is dominated by Salix caprea, Populus tremula, Betula pendula or Sorbus aucuparia into the alliance Sambuco-Salicion capreae (order Sambucetalia racemosae and class Rhamno-Prunetea) - Exner \& Willner (2007). In Slovenia, mountain ash (Sorbus

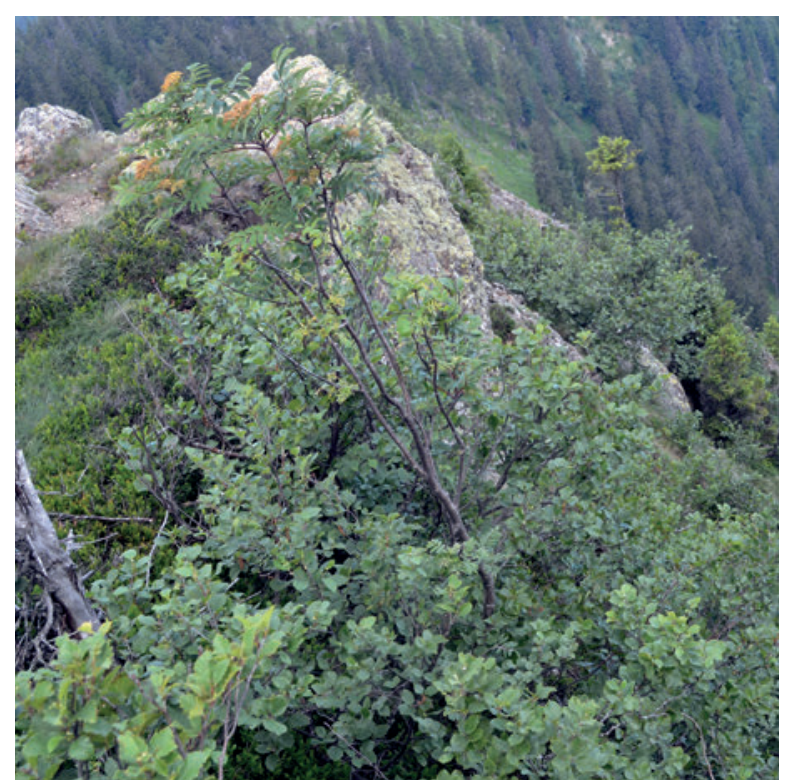

Stand of the association Huperzio selagi-Alnetum viridis on northern slopes of Mt. Komen (Kamen) in the Smrekovec Mountains.

Sestoj asociacije Huperzio selagi-Alnetum viridis na severnih pobočjih Komna (Kamna) v Smrekovškem pogorju.

aucuparia) is found mainly admixed in beech, firbeech, spruce and larch forests in submontane, montane and subalpine belt. It is distributed in most of the country (it is rare only in the Primorje region, in the Karst and Istria, as well as in southeastern Slovenia and the Prekmurje region) - Figure 1. Distribution of both subspecies (subsp. aucuparia and subsp. glabrata), has not been so well researched. According to the data in the FloVegSi database, the subspecies Sorbus aucuparia subsp. glabrata grows in the Alps (the Julian and the Kamnik-Savinja Alps, the Karavanke Mts., Pohorje) and on the Trnovski gozd plateau. Our findings have shown that, at least in the Julian Alps, this is a very rare subspecies and that even in the subalpine communities, larch stands, dwarf pine and green alder stands, the typical form, Sorbus aucuparia subsp. aucuparia, prevails. Only the typical subspecies was determined also in the case of the described pioneer stage. M. Wraber (1960) mentions mountain ash as an important tree species in two montane communities on Pohorje, in maple forests Sorbo aucupariae-Aceretum pseudoplatani and in spruce forests Sorbo aucupariae-Piceetum. Its pioneer stands are also likely to be found here. Mountain ash and green alder stands are known also elsewhere in the Alps. ETH 
Zürich students (Hari, Leisinger \& Zysset 1993) pointed to the occurrence of a similar mountain ash community in Switzerland in 1993, but did not back that up with further publication. Steiger (1994: 96, 2010: 129) in his work on Swiss forests mentions the association Alno viridis-Sorbetum aucupariae prov., which indicates it has not yet been described according to the rules of the Code of Phytocoenological Nomenclature (Weber et al. 2000). This article aims to compensate for that. Since we do not have access to the published relevés of this community elsewhere in the Alps, we selected as its nomenclature type, holotypus, a relevé from our table, i.e. relevé No. 6 in Table 6. Diagnostic species of the new association are Sorbus aucuparia, Salix appendiculata, Alnus viridis, Senecio cacaliaster and Veratrum album subsp. lobelianum. The new association is classified into the alliance Alnion viridis, order Alnetalia viridis and class Betulo-Alnetea. Such classification is justified with the domination of the species of this class and tall herbs from the class Mulgedio-Aconitetea. There is no sound basis that would justify classification of the new association into the alliance Sambuco-Salicion capreae and class Rhamno-Prunetea on the grounds of its floristic composition. These stands are not common in the Julian Alps and their foothills. So far, they have been recorded on the northern slopes of Matajur, on Sanek above Stržišče in the Bača Valley and under Mts. Kobla and Črna gora in Bohinj (Figure 6), at the elevation between $1300 \mathrm{~m}$ and $1600 \mathrm{~m}$, in montane and very humid climate, with mean annual precipitation of between $2200 \mathrm{~mm}$ and $2800 \mathrm{~mm}$. The new association is divided into two subassociations. The subassociation Alno viridis-Sorbetum aucupariae adenostyletosum glabrae subass. nova hoc loco characterises steep, shady, stony limestone sites with rendzina or shallow brown calcareous soil with frequent snowslides. The nomenclature type, holotypus, of this subassociation is the nomenclature type of the new association (relevé No. 6 in Table 6). Differential species of the subassociation are Adenostyles glabra, Asplenium viride, Cystopteris fragilis and Soldanella alpina. The subassociation Alno viridis-Sorbetum aucupariae luzuletosum sylvaticae subass. nova hoc loco characterises pioneer stages on former hay meadows on mixed geological bedrock, limestone, marlstone, claystone and chert on slightly acid (dystric) soil. Its nomenclature type, holotypus, is relevé No. 1 in Table 6 and its differential species are Luzula sylvatica and Sorbus chamaemespilus.

\subsubsection{Alno viridis-Aceretum pseudoplatani nom. prov.}

In Table 7 we publish the relevé made in the cirque Komar under Kanjavec, above Zadnjica in the Trenta at the elevation of around $1430 \mathrm{~m}$ (Figure 6). Here, on a steep prominence between two gorges with frequent snowslides, grows a low open sycamore maple forest of coppice and sabre growth, reaching a tree height of 10 metres and diameters at breast height of up to $30 \mathrm{~cm}$. The shrub layer that covers almost the same area as the tree layer is dominated by green alder (Alnus viridis) and large-leaved willow (Salix appendiculata). The herb layer comprises species of tall herbs, beech, spruce and basophilous pine forests. With its floristic composition this stand resembles above all the green alder forest from the association Rhododendro hirsuti-Alnetum viridis - in any respect more than it resembles montane sycamore maple communities from associations Lamio orvalae-Aceretum and Aconito paniculati-Fagetum (compare P. Košir 2005, Dakskobler 2007 and Zupančič 2012). We believe this is a long-term successional stage whose development into a higher stage - at this elevation in the Primorje part of the Julian Alps this means subalpine beech forest (Polysticho lonchitis-Fagetum) - is thwarted by natural conditions, a stony moist site and annual snowslides. As we must consider the highest stand layer of sycamore maple, this relevé is classified into, for the time being only provisional, new association Alno viridis-Aceretum pseudoplatani nom. prov. A valid description of this association will be possible if we can find similar stands elsewhere. For now, the provisional new association is classified into the alliance Alnion viridis and class Betulo-Alnetea viridis.

\subsubsection{Polysticho lonchitis-Fagetum}

Authors of recent phytosociological literature (Accetto 2002, Surina \& Rakaj 2007, Willner 2007, Marinček \& Čarni 2010, Zupančič 2012) have different views of this association; some of them do not see it as independent and classify it into more widely interpreted altimontane beech community of the eastern and southeastern Alps and the northern part of the Dinaric Mountains (Saxifrago rotundifolii-Fagetum Zukrigl 1989 or Ranunculo platanifolii-Fagetum Marinček et al. 1993). In our opinion, based on around 400 rel- 


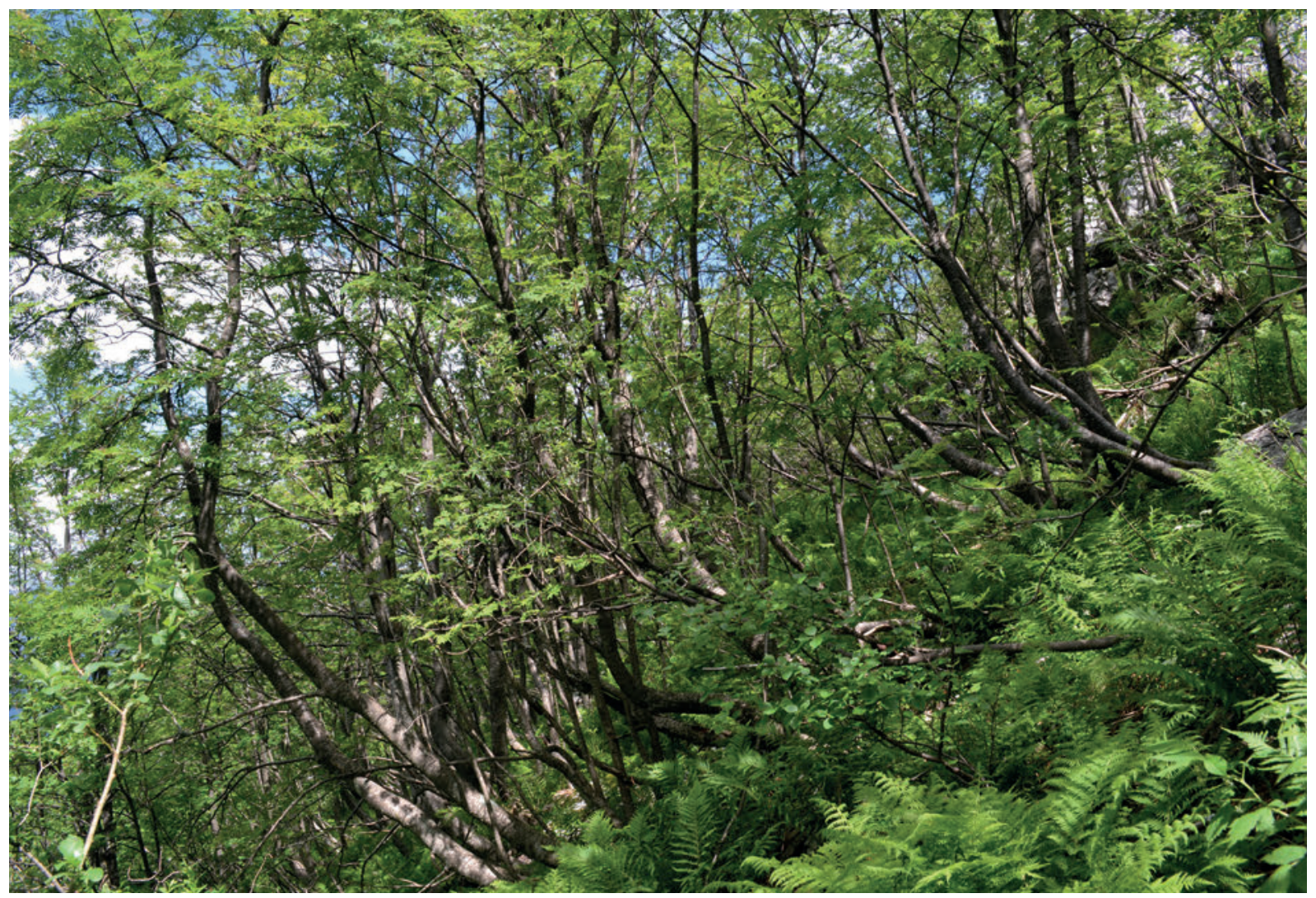

Stand of the association Alno viridis-Sorbetum aucupariae on northern slopes of Mt. Matajur.

Sestoj asociacije Alno viridis-Sorbetum aucupariae na severnih pobočjih Matajurja.

evés from the Julian Alps and the Trnovski gozd plateau, independence of this association is justified not only in terms of its sites and physiognomy - the elevation belt immediately along the existing timberline with low, usually clustered and coppice beech (polycormon tree form) - but also floristically, with a number of differential species, especially character species of subalpine scrub communities, tall herbs and grasslands. Such good differential species that rarely, or not at all, grow in other beech communities are Rhododendron hirsutum, Sorbus chamaemespilus, Lonicera caerulea, Salix appendiculata, Pinus mugo, Clematis alpina, Senecio cacaliaster, Aster bellidiastrum, Laserpitium peucedanoides, Festuca calva and Astrantia bavarica, in our case also Alnus vir$i d i s$ and others. While it is true that some of the above-mentioned species may be diagnostic also for some other beech associations such as Rhododendro hirsuti-Fagetum or Aconito paniculati-Fagetum, the ecological conditions and entire species composition in these cases are considerably different. Our material on the association Polysticho
lonchitis-Fagetum still needs to be processed, but this article publishes 27 relevés of beech forests at the timberline in the Julian Alps (Table 8), most of which (possibly excluding relevés No. 26 and 27) can undoubtedly be classified into this association. These beech stands comprise also green alder, which indicates the specifics of their natural structure. These stands occur on extreme sites with an open tree layer, which is mainly the consequence of natural factors - steep slopes, erosion, snowslides. In subalpine beech forests the green alder was recorded at the elevation of $1360 \mathrm{~m}$ to $1580 \mathrm{~m}$ a.s.l., geological bedrock is limestone, only rarely admixed with dolomite or marlstone, the soil is rendzina. Steep to very steep shady slopes prevail. Such beech stands were found in the southwestern foothills of the Julian Alps, under the ridge of Stol, in Resia, in the Krn Mts., in the ridges of Bavški Grintavec and Loška stena, under the Tolmin-Bohinj ridge and under Porezen (the stands under Porezen are floristically closer to the association Ranunculo platanifolii-Fagetum). 


\subsubsection{Rhodothamno-Laricetum}

Regardless of the prevailing calcareous bedrock, green alder is a relatively common species in the scrub layer of the eastern-Alpine larch community. This is due to mainly favourable light conditions under the open tree layer and to the fact that natural larch forests frequently grow on steep shady slopes with very moist soil where organic matter accumulates. From extensive material (more than 330 relevés) we selected 40 relevés where green alder's cover value in the shrub layer is 1 or more (Table 9). Such larch stands were recorded in the Julian Alps, the Kamnik-Savinja Alps and the Karavanke Mts. They were made at elevation of $1410 \mathrm{~m}$ to $1830 \mathrm{~m}$ a.s.l., geological bedrock is limestone or dolomite limestone, only exceptionally is limestone admixed with marlstone; soils are moder rendzinas. The aspect is almost always shady and the slope usually very steep. Relevé No. 40 stands out from the other relevés; it was made under Srednji vrh in the Karavanke Mts. where geological bedrock consists of claystone and the soil is brown, eutric. This relevé is not classified into the association Rhodothamno-Laricetum, but is temporarily treated as a syntaxon Alno viridisLaricetum deciduae nom. prov. Supposedly, this is a successional stage on former non-forest land where the larch forest gradually replaces the pioneer green alder scrub (Alnetum viridis). At the moment, other stands cannot be treated as a special subassociation Rhodothamno-Laricetum alnetosum viridis, even though green alder clearly characterises special, cold and moist sites. This is due to similar reasons as in dwarf mountain-pine (Pinus mugo). These two species are more or less abundant in larch forests and relevés do not usually group only based on their presence or absence, but also according to other factors. Green alder was nevertheless identified as a diagnostic species of lower-level units, similarly to Luzula nivea and Stellaria montana which may indicate two different variants of the studied association.

\subsubsection{Rhododendro hirsuti-Pinetum mugo (= Rho- dothamno-Pinetum mugo Zupančič et Žagar in Zupančič 2013)}

Green alder occurs relatively frequently also in $\mathrm{Al}-$ pine dwarf mountain-pine stands. In the territory of Slovenia this applies not only to relevés published by Zupančič et al. (2006), where in the ana- lytic table green alder demonstrates class of constancy 2 (frequency $32 \%$ ), but also to our mainly still unpublished relevés (about 80). Among them we identified only those where green alder has a cover value 1 or more (Table 10). These relevés were made in the Julian Alps at elevation of $1360 \mathrm{~m}$ to $1770 \mathrm{~m}$ a.s.l., on limestone and dolomite bedrock or on talus slopes, usually on very steep shady slopes with persistent snow cover. The soils are moder rendzinas. For now, this form of Alpine dwarf mountain-pine stands is treated as a special variant Rhododendro hirsuti-Pinetum mugo typicum Zupančič, Žagar \& Culiberg 2006 var. Alnus viridis = Rhodothamno-Pinetum mugo Zupančič et Žagar in Zupančič 2013 var. Alnus viridis (see also Zupančič 2013). It can be described as one of the most hygrophilous forms of Alpine dwarf mountain-pine stands in Slovenia. Here, the species Alnus viridis, Salix appendiculata, S. glabra and S. waldsteiniana indicate a certain similarity with subalpine willow communities (Aceri-Salicetum appendiculatae). A similar subalpine community in which green alder acts as a diagnostic species was described in the Julian Alps, i.e. the association Rhododendro hirsuti-Betuletum carpaticae (Dakskobler et al. 2012). Its sites are shady glacial cirques with annual snowslides that obstruct successional development into subalpine beech forest. The open tree layer is dominated by Carpathian birch (Betula pubescens subsp. carpatica), and the shrub layer by Pinus mugo, Rhododendron hirsutum and Alnus viridis.

\subsection{COMPARISON OF GOMMUNITIES WITH ALNUS VIRIDIS IN Slovenia ACGORDING TO LANDOLT'S INDICATOR VALUES}

Results of the phytoindication analysis in the stands with green alder that was carried out using ecological indicator values (Landolt et al. 2010) are presented in Figures 7 and 8.

Calculated temperature values $(\mathrm{T})$ indicate slightly more favourable temperature conditions in the stands of the syntaxa Alno viridis-Sorbetum aucupariae, Alnetum viridis typicum and Polysticho lonchitis-Fagetum in comparison with the stands of the syntaxa Rhododendro hirsuti-Pinetum mugo var. Alnus viridis and Rhodothamno-Laricetum. Continentality $(\mathrm{K})$ is the least pronounced in the stands of the subassociation Alnetum viridis typicum and the most pronounced in the stands with dominant conifers (larch and dwarf pine stands). Similarly, light conditions (L) are more favourable in conifer 


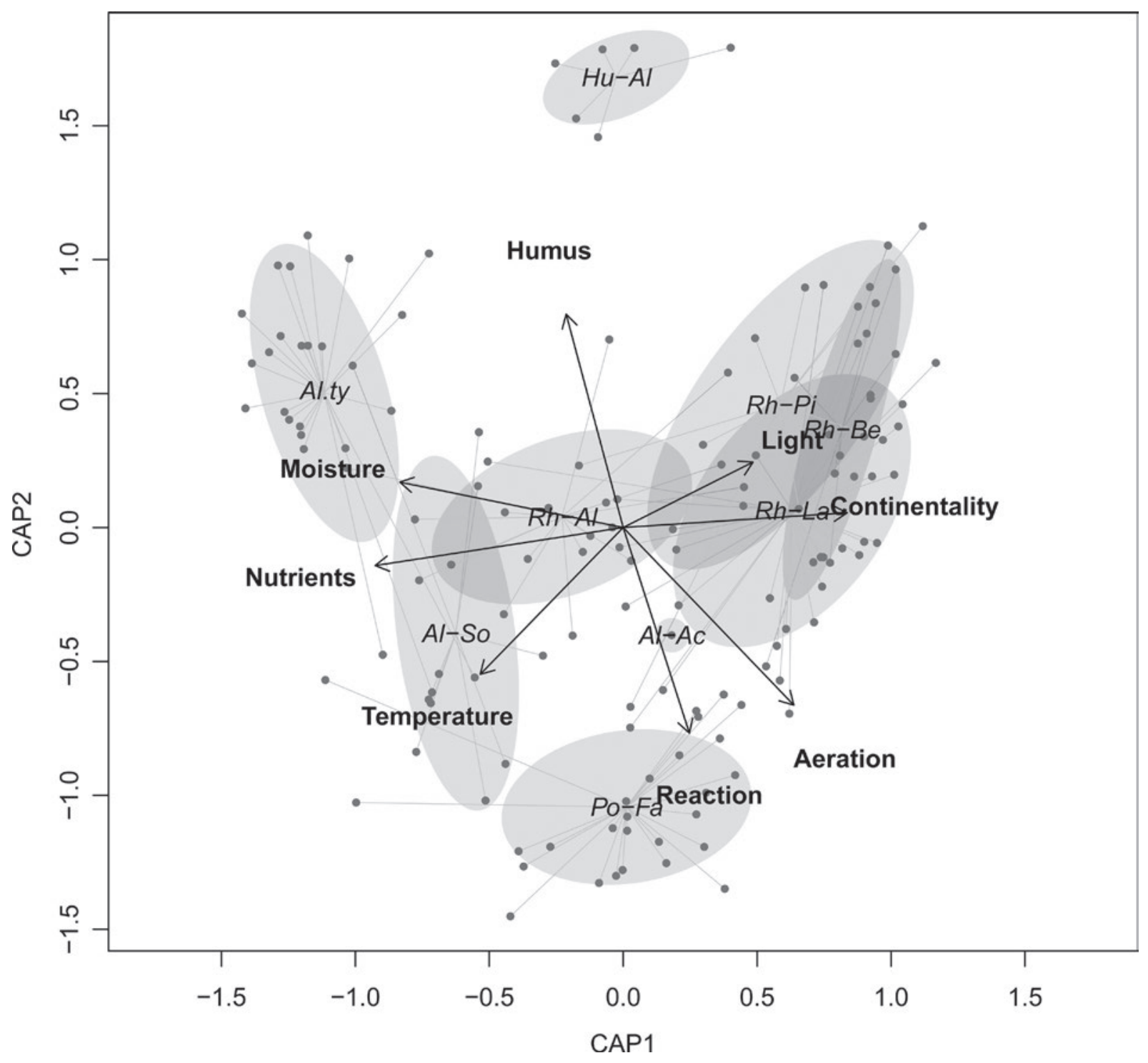

Al-Ac Alno viridis-Aceretum pseudoplatani

Al-So Alno viridis-Sorbetum acupariae

Alty. Alnetum viridis typicum

Hu-Al Huperzio selagi-Alnetum viridis

PoFa Polysticho lonchitis-Fagetum

RhAl Rhododendro hirsuti-Alnetum viridis

RhBe Rhododendro hirsuti-Betuletum carpaticae

Rh-La Rhodothamno-Laricetum

$\mathrm{Rh}-\mathrm{Pi}$ Rhododendro hirsuti-Pinetum mugo var. Alnus viridis

Figure 7: The ordination plot of the first and second CAP axes of vascular plant coverage in different syntaxa with Alnus viridis. All eight constrained axes explain $41,65 \%$ of total variation, first two explain $18,33 \%$ and $9,27 \%$ respectively. Arrows represent Landolt's indicator values.

Slika 7: Ordinacijski diagram prve in druge osi Kanonične analize glavnih koordinat (CAP) zastiranja praprotnic in semenk v različnih združbah z vrsto Alnus viridis. Kanonične osi (vseh osem skupaj) pojasnijo 41,65\% skupne variabilnosti, narisani prvi dve osi pojasnita 18,33\% oz. 9,29\% variabilnosti. Puščice predstavljajo Landoltove indikacijske vrednosti.

stands. Moisture (M) is the highest in the stands of the syntaxa Alnetum viridis typicum and Rhododendro hirsuti-Alnetum viridis where green alder dominates. Calculated soil reaction values $(\mathrm{R})$ indicate considerably higher soil acidity in the stands of the association Huperzio-Alnetum viridis that occurs on silicate rocks and the sites of the subassociation Alnetum viridis typicum also demonstrate higher acidity than other communities. The highest mean values for the soil reaction indicator (R) 

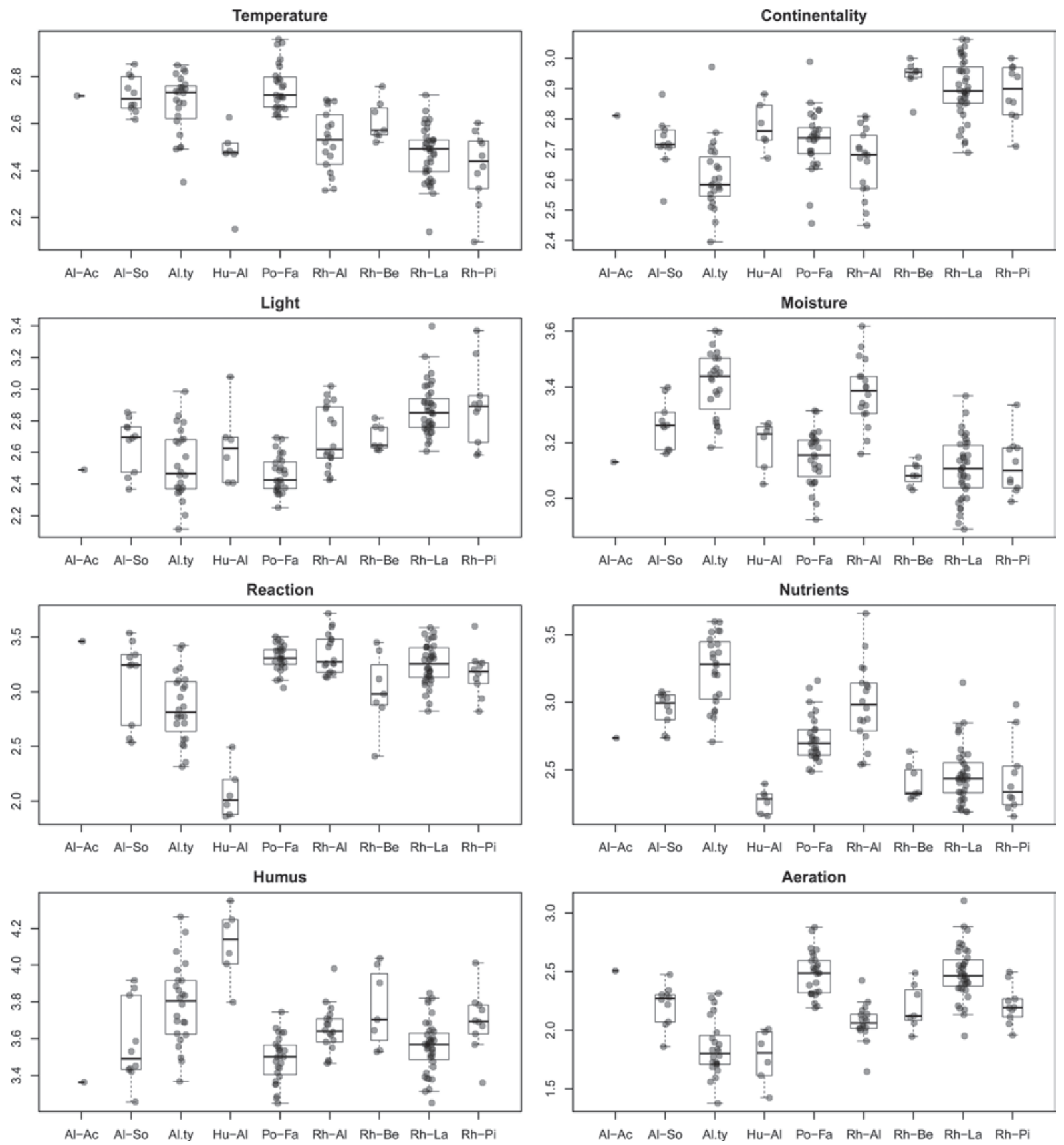

Figure 8: Comparison of ecological conditions in the communities with Alnus viridis, determined with Landolt's indicator values. Slika 8: Primerjava ekoloških razmer v združbah z vrsto Alnus viridis, ugotovljenih s pomočjo Landoltovih fitoindikacijskih vrednosti.

were calculated in the stands of the associations Polysticho-Fagetum and Rhododendro hirsuti-Alnetum viridis. Calculated nutrient values $(\mathrm{N})$ indicate better nutrient supply on the sites of the syntaxa Alnetum viridis typicum, Rhododendro hirsuti-Alnetum viridis and Alno viridis-Sorbetum aucupariae, while the sites of the association Huperzio-Alnetum vir$i d i s$ and larch and dwarf pine communities are the most nutrient-poor. Among the compared communities the sites of the syntaxa Huperzio-Alnetum viridis and Alnetum viridis typicum have the highest humus content in soils $(\mathrm{H})$ and the poorest soil aeration (A). Phytoindication demonstrates reverse conditions in terms of humus content and soil aeration in the stands of the associations Polysticho-Fagetum and Rhodothamno-Laricetum. 


\section{CONCLUSIONS}

Green alder (Alnus viridis subsp. viridis) is an important species of our montane forests. As a pioneer species it has established itself in the predominantly limestone Slovenian Alps mainly on steep shady slopes, in gullies and hollows where snowslides occur every year and the snow cover lingers long into the spring. On such sites it forms long-term successional stages and it is unlikely to be replaced by the surrounding forest growth (most often subalpine larch or spruce forest) that occurs on slopes that are less exposed to avalanches. Such, more or less primary green alder stands on calcareous bedrock in the Eastern and Southeastern Alps are classified into the new association Rhododendro hirsuti-Alnetum viridis. It is differentiated from other communities described in the Alps (Alnetum viridis s. str., Rhododendro ferruginei-Alnetum viridis) by a higher number of mainly calciphilous species such as Rhododendron hirsutum, Sorbus chamaemespilus, Polystichum lonchitis, Valeriana tripteris, Asplenium viride, Aconitum lycoctonum s. lat., Galeobdolon flavidum, Thalictrum aquilegiifolium, Salix waldsteiniana, Adenostyles glabra, Cystopteris montana, Homogyne sylvestris, Acer pseudoplatanus, Primula elatior and others. Floristically slightly different are the pioneer green alder stands on abandoned pastures and mountain pastures that were cleared mainly in the belt of altimontane and subalpine beech and very rarely also spruce forests from the associations Ranunculo platanifolii-Fagetum, Polysticho lonchitis-Fagetum, Luzulo-Fagetum abietetosum and Luzulo sylvaticaePiceetum. Such stands, if they grow on limestone bedrock and on stony sites, are partly still classified into the association Rhododendro hirsuti-Alnetum viridis (several stands on the northern slopes of Porezen), and others into the syntaxon Alnetum viridis typicum (pioneer alder stands on silicate bedrock in the Karavanke Mts.) or merely into the wider macroassociation Alnetum viridis s. lat. (younger successional stages in the southern foothills of the Julian Alps). Floristically distinct is the green alder community in the shady andesite rocks under the peak of Komen in the Smrekovec Mts. (eastern Kamnik-Savinja Alps) that is classified into the new association Huperzio selagi-Alnetum viridis and represents vegetation that is unique in the silicate Alps. Here, the stands on similar sites are classified into the association Rhododendro ferruginei-Alnetum viridis. This classification was not possible in our case as Rhododendron ferrugineum was not spotted under Komen (Kamen); likewise, some diagnostic species of the classes Betulo-Alnetea viridis and Mulgedio-Aconitetea do not grow in our stands. They are dominated by species of spruce forests from the class Vaccinio-Piceetea, including Vaccinium vitis-idaea, Huperzia selago, Lycopidum annotinum and Thelypteris limbosperma, some character species of silicate rocks or silicate subalpine grasslands (Primula villosa, P. minima) and somes mosses (Sphagnum russowii, Rhytidiadelphus loreus). In the Julian Alps we found stands where green alder was partly replaced in succession by mountain ash. Similar stands are known elsewhere in the Alps, but have not yet been validly described as the new association Alno viridisSorbetum aucupariae, which is what we did in this article. A stand with dominant sycamore maple and green alder was provisionally described as the new association Alno viridis-Aceretum pseudoplatani nom. prov. The phytosociological table presented also the subalpine beech community (Polysticho lonchitis-Fagetum) where green alder grows due to the natural open tree layer and the subalpine larch community (Rhodothamno-Laricetum) where green alder in the shrub layer has a cover value of 1 or more. The larch relevé with green alder on claystone and eutric brown soil under Srednji vrh in the Karavanke Mts. is treated as a successional stage Alno viridis-Laricetum deciduae nom. prov. This species can be similarly abundant in a special form of eastern-Alpine dwarf mountain-pine community (Rhododendro hirsuti-Pinetum mugo typicum Zupančič, Žagar \& Culiberg 2006 var. Alnus viridis = Rhodothamno-Pinetum mugo Zupančič et Žagar in Zupančič 2013 var. Alnus viridis) and in the community of dwarf mountain-pine, hairy alpenrose and Carpathian birch (Rhododendro hirsuti-Betuletum carpaticae).

Green alder stands and communities play a significant ecological and protective role in our Alps and their foothills. In last decades we have been observing their deterioration, mainly as a result of fungal diseases and pest damage. One of the agents frequently seen as responsible for the withering of green alder is Cryptodiaporthe oxystoma (Pisetta et al. 2012) that belongs to the fungal group Ascomycota, detected and determined in Slovenia (for example in the stands under Porezen and Kobilja glava) by G. Seljak and G. Podgornik (in litt.). For now, withering of alder has not been detected on a larger scale on other locations. Researchers attribute this alarming phenomenon to climate change and decreasing snow cover. 


\section{POVZETEK}

\section{Gozdne in grmiščne združbe $z$ zeleno jelšo (Alnus viridis) v Sloveniji}

Zelena jelša (Alnus viridis subsp. viridis) je pomembna vrsta naših gorskih gozdov. Kot pionirska vrsta se v pretežno apnenčastih slovenskih Alpah uveljavlja predvsem na strmih osojnih pobočjih, $\mathrm{v}$ žlebovih in kotanjah, kjer vsako leto polzijo snežni plazovi in se snežna odeja zadržuje dolgo v pomlad. Na takih rastiščih oblikuje dolgotrajne sukcesijske stadije, in je malo možnosti, da bi jo nadomestilo okoliško gozdno rastje (največkrat subalpinski macesnov ali smrekov gozd), ki uspeva na manj plazovitih pobočjih. Takšno bolj ali manj primarno zelenojelševje na karbonatni podlagi v vzhodnih in jugovzhodnih Alpah uvrščamo v novo asociacijo Rhododendro hirsuti-Alnetum viridis. Od drugih v Alpah opisanih združb (Alnetum viridis s. str., Rhododendro ferruginei-Alnetum viridis) jo razlikuje večje število večinoma kalcifilnih vrst, kot so Rhododendron hirsutum, Sorbus chamaemespilus, Polystichum lonchitis, Valeriana tripteris, Asplenium viride, Aconitum lycoctonum s. lat., Galeobdolon flavidum, Thalictrum aquilegiifolium, Salix waldsteiniana, Adenostyles glabra, Cystopteris montana, Homogyne sylvestris, Acer pseudoplatanus, Primula elatior in druge. Floristično nekoliko drugačni so pionirski sestoji zelene jelše na opuščenih pašnikih in planinah, ki so bili izkrčeni $\mathrm{v}$ glavnem $\mathrm{v}$ pasu altimontanskih in subalpinskih bukovih in zelo redko tudi smrekovih gozdov iz asociacij Ranunculo platanifolii-Fagetum, Polysticho lonchitis-Fagetum, Luzulo-Fagetum abietetosum, Luzulo sylvaticae-Piceetum. Takšne sestoje, če uspevajo na apnenčasti podlagi in na kamnitih rastiščih, deloma še uvrščamo $\mathrm{v}$ asociacijo Rhododendro hirsuti-Alnetum viridis (nekateri sestoji na severnih pobočjih Porezna, pod Koblo in Slatnikom), druge pa v sintakson Alnetum viridis typicum (pionirski zelenojelševi sestoji na silikatni podlagi v Karavankah) ali zgolj v široko zajeto makroasociacijo Alnetum viridis s. lat. (mlajši sukcesijski stadiji v južnem prigorju Julijskih Alp). Floristično zelo posebno je zelenojelševje $\mathrm{v}$ osojnem andezitnem skalovju pod vrhom Komna v Smrekovškem pogorju (vzhodne Kamniško-Savinjske Alpe), ki ga uvrščamo v novo asociacijo Huperzio selagi-Alnetum viridis in je vegetacijska posebnost $\mathrm{v}$ silikatnih Alpah. V njih sestoje na podobnih rastiščih namreč uvrščajo v asociacijo Rhododendro ferruginei-Alnetum viridis. Takšna uvrstitev $\mathrm{v}$ našem prim- eru ni bila mogoča, saj pod Komnom (Kamnom) vrste Rhododendron ferrugineum nismo opazili, prav tako v naših popisih ne uspevajo nekatere diagnostične vrste razredov Betulo-Alnetea viridis in Mulgedio-Aconitetea. V njih prevladujejo vrste smrekovih gozdov iz razreda Vaccinio-Piceetea, med njimi tudi vrste Vaccinium vitis-idaea, Huperzia selago, Lycopidum annotinum in Thelypteris limbosperma, nadalje nekatere značilnice silikatnega skalovja ali silikatnih subalpinskih travišč (Primula villosa, $P$. minima) ter nekateri mahovi (Sphagnum russowii, Rhytidiadelphus loreus). V Julijskih Alpah smo našli tudi sestoje, kjer je zeleno jelšo v sukcesiji deloma nadomestila jerebika. Podobne sestoje poznajo tudi drugod v Alpah, a do zdaj še niso bili veljavno opisani kot nova asociacija Alno viridis-Sorbetum aucupariae, zato smo to storili v tem članku. Sestoj s prevladujočima gorskim javorjem in zeleno jelšo pa uvrščamo v le provizorno opisano novo asociacijo Alno viridis-Aceretum pseudoplatani. $\mathrm{S}$ fitocenološko tabelo smo predstavili tudi subalpinsko bukovje (Polysticho lonchitis-Fagetum), v katerem zaradi naravnega vrzelastega drevesnega sklepa uspeva tudi zelena jelša, in subalpinsko macesnovje (Rhodothamno-Laricetum), v katerem ima zelena jelša $\mathrm{v}$ grmovni plasti oceno zastiranja 1 ali več. Popis macesnovja $\mathrm{z}$ zeleno jelšo na glinavcu in evtričnih rjavih tleh pod Srednjim vrhom v Karavankah vrednotimo kot sukcesijski stadij Alno viridis-Laricetum deciduae nom. prov. Podobno obilna je ta vrsta lahko v posebni obliki vzhodnoalpskega ruševja (Rhododendro hirsutiPinetum mugo typicum Zupančič, Žagar \& Culiberg 2006 var. Alnus viridis = Rhodothamno-Pinetum mugo Zupančič et Žagar in Zupančič 2013 var. Alnus viridis) in v združbi rušja, dlakavega sleča in karpatske breze (Rhododendro hirsuti-Betuletum carpaticae).

Sestoji in združbe zelene jelše imajo $\mathrm{v}$ naših Alpah s prigorjem važno ekološko in varovalno vlogo. V zadnjih desetletjih tudi v Sloveniji opažamo njihovo propadanje, predvsem kot posledica glivičnih bolezni in napadov insektov. Med povzročitelji sušenja zelene jelše se pogosto omenja vrsto Cryptodiaporthe oxystoma (Pisetta et al. 2012), glivo iz skupine zaprtotrosnic (Ascomycota), ki sta jo pri nas (na primer v sestojih pod Poreznom in Kobiljo glavo) opažala in določala G. Seljak in G. Podgornik (in litt.). Za zdaj razen na teh dveh gorah znakov jelšinega sušenja $\mathrm{v}$ večjem obsegu na drugih lokacijah še nismo opazili. Ta zaskrbljujoč pojav raziskovalci povezujejo $\mathrm{z}$ podnebnimi spremembami in manjšo količino snežne odeje. 


\section{ACKNOWLEDGEMENTS}

We are sincerely grateful to the first researcher of green alder stands on Porezen, mag. Gabrijel Seljak, whose relevés that were published in his graduation thesis were of valuable assistance to us (however, due to the radical changes in the vegetation under Porezen these relevés could not be repeated). Gabrijel Seljak and Gregor Podgornik pointed out the withering and deterioration of green alder stands and their causes. We would also like to thank Prof. Dr. Andrej Martinčič for the determination of moss species on the relevés from the Smrekovec Mts., which significantly contributed to the description of the new association. Sincere thanks to Dr. Branko Vreš for joining us in the field in the eastern Karavanke Mts. and Branko Zupan for coming with us to the Stara Fužina pasturelands. We are especially grateful to Prof. Dr. Livio Poldini who kindly provided, before publication, the article in which he and his colleagues gave a synthetic presentation of green alder communities in the entire region of the Alps; to Prof. Dr. Robert Brus for pointing out Paulin's article from 1915; to Prof. Dr. Jean-Paul Theurillat for his clarifications on the validity of the description of the association Alno viridis-Sorbetum aucupariae; to Prof. Dr. Jurij Diaci and Prof. Dr. Monika Frehner for the data on this community in Switzerland and to Prof. Dr. Sead Vojniković for the literature he sent on the green alder community in Bosnia and Herzegovina. Two anonymous reviewers helped us with valuable improvements and corrections. English translation by Andreja Šalamon Verbič.

\section{REFERENCES}

Accetto, M. 2002: Pragozdno rastlinje rezervata Krokar na Kočevskem. Gozdarski vestnik 60 (10): 419-443.

Aeschimann, D., Lauber, K., Moser, D. M. \& Theurillat, J.-P. 2004: Flora alpina. Bd. 1: Lycopodiaceae-Apiaceae. Haupt Verlag, Bern, Stuttgart, Wien, 1159 pp.

Aichinger, E. 1933: Vegetationskunde der Karawanken. Gustav Fischer, Jena, 329 pp.

Anderson, M. J. \& Willis, T. J. 2003: Canonical analysis of principal coordinates: a useful method of constrained ordination for ecology. Ecology 84 (2): 511-525.
Bakan, B. 2006: Slikovni pregled višjih rastlin Prekmurja. Prispevek k poznavanju flore Prekmurja. Razvojni center Lendava, 245 pp.

Ball, P. W. 1993: Alnus Miller. In: Tutin, T. G., Heywood, V. H., Burges, N. A., Valentine, D. H., Walters, S. M. \& Webb, D. A. (eds.): Flora Europaea, Volume 1: Lycopodiaceae to Platanaceae (ed. 2), Cambridge, pp. 69-70.

Boscutti, F., Poldini, L. \& Buccheri, M. 2013: Green alder communities in the Alps. Phytosociological variability and ecological features. Plant Biosystems 147 (in print). DOI: 10.1080/11263504.2013.809813

Braun-Blanquet, J. 1964: Pflanzensoziologie. Grundzüge der Vegetationskunde. 3. Auflage. Springer, Wien - New York, 865 pp.

Braun-Blanquet, J. 1973: Zur Kenntnis der Vegetation alpiner Lawinenbahnen. Mitteilungen der Floristisch-soziologischen Arbeitsgemeinschaft N. F. Heft 15/16: 146-152, TodenmannGöttingen.

Brus, R. 2008: Sto grmovnih vrst na Slovenskem. Tehniška založba Slovenije, Ljubljana, 215 pp.

Buser, S. 2009: Geološka karta Slovenije $1: 250.000$. Geological map of Slovenia 1.250,000. Geološki zavod Slovenije, Ljubljana.

Čolić, D., Mišić, V. \& Popović, M. 1963: Fitocenološka analiza visokoplaninske zajednice šleske vrbe i planinske jove (Saliceto-Alnetum viridis ass. nova) na Staroj planini. Zbornik radova, Biološki institut NR Srbije 6 (5): 3-43.

Dakskobler, I. 2006: Asociacija RhodothamnoLaricetum (Zukrigl 1973) Willner \& Zukrigl 1999 v Julijskih Alpah. The Association Rhodothamno-Laricetum (Zukrigl 1973) Willner \& Zukrigl 1999 in the Julian Alps. Razprave 4. razreda SAZU 47-1: 117-192.

Dakskobler, I. 2007: Gozdovi plemenitih listavcev v Posočju. Forest of valuable broad-leaved tree species in the Soča valley (western Slovenia). Scopolia 60: 1-287.

Dakskobler, I., Rozman, A. \& Franz, W. R. 2012: Betula pubescens Ehrh. subsp. carpatica (Willd.) Ascherson \& Graebner, a new taxon in the flora of the Julian Alps and Slovenia and its new association Rhododendro hirsuti-Betuletum carpaticae ass. nov. Folia biologica et geologica $53(1-2): 5-23$.

Exner, A. \& Willner, W. 2007: Rhamno-Prunetea Rivas Goday \& Borja Carbonell ex Tx 1962. In: Willner, W. \& Grabherr, G. (eds.): Die Wälder und Gebüsche Österreichs. Ein Bestimmungswerk mit Tabellen. 1. Textband. 
Spektrum Akademischer Verlag in Elsevier, Heidelberg, pp. 62-83.

Fleischmann, A. 1844: Übersicht der Flora Krain's. Ann. Landwirth.-Ges. Krain 6: 103246 Ljubljana.

Hari - Leisinger-Zysset, 1993: Der Vogelbeerwald an der Waldgrenze im Gebiet Monte LemaMonte Gradiccioli-Monte Tamaro, Malcantone TI. Studienarbeit Abt. Umweltnaturwissenschaften ETH, Zürich.

Jurkovšek, B. 1987 a: Tolmač listov Beljak in Ponteba. Osnovna geološka karta SFRJ 1:100 000. Zvezni geološki zavod, Beograd, 58 pp.

Jurkovšek, B. 1987 b: Osnovna geološka karta SFRJ. Beljak in Ponteba 1: 100 000. Zvezni geološki zavod, Beograd.

Juvan, N., Čarni, A. \& Jogan, N. 2011: Chasmophytic vegetation of silicate rocks on the southern outcrops of the Alps in Slovenia. Wulfenia 18: 133-156.

Karner, P. 2007 a: Betulo-Alnetea viridis prov. In: Willner, W. \& Grabherr, G. (eds.): Die Wälder und Gebüsche Österreichs. Ein Bestimmungswerk mit Tabellen. 1. Textband. Spektrum Akademischer Verlag in Elsevier, Heidelberg, pp. 83-88.

Karner, P. 2007 b: Betulo-Alnetea viridis prov. In: Willner, W. \& Grabherr, G. (eds.): Die Wälder und Gebüsche Österreichs. Ein Bestimmungswerk mit Tabellen. 2. Tebellenband. Spektrum Akademischer Verlag in Elsevier, Heidelberg, pp. 47-52.

Košir, P. 2005: Noble hardwood forests of the altimontane belt (Lamio orvalae-Aceretum pseudoplatani P. Košir et Marinček 1999) in Slovenia (western part of the Illyrian floral province). Natura Croatica 14 (2): 59-86.

Landolt, E., Bäumler, B. Erhardt, A., Hegg, O., Klötzli, F., Lämmler, W., Nobis, M., RudmannMaurer, K., Schweingruber, F. H., Theurillat, J.-P., Urmi, E., Vust, M. \& Wohlgemuth, T. 2010: Flora indicativa. 2. Auflage. Haupt Verlag, Bern-Stuttgart-Wien, 323 pp.

Lovrenčak, F., Strmole, D., Wraber, T. Kolar, I. \& Ževart, M. 1998: Smrekovško pogorje. In: Voglar, D. (ed.): Enciklopedija Slovenije 12, Mladinska knjiga, Ljubljana: pp. 91.

Maarel, van der E. 1979: Transformation of coverabundance values in phytosociology and its effects on community similarity. Vegetatio 39 (2): 97-114.

Marinček, L. \& Čarni, A. 2010: Altimontanski bukovi gozdovi podzveze Saxifrago-Fagenion (Aremonio-Fagion). Scopolia 69: 1-107.
Martinčič, A. 2003: Seznam listnatih mahov (Bryopsida) Slovenije. Hacquetia 2 (1): 91-166.

Martinčič, A. 2008: Mahovna flora Smrekovškega pogorja (Kamniško-Savinjske Alpe, Slovenija). Hacquetia 7 (1): 33-46.

Martinčič, A. 2011: Annotated Checklist of Slovenian Liverworts (Marchanthiophyta) and Hornworts (Anthocerotophyta). Scopolia 72: 1-38.

Martinčič, A., Wraber, T., Jogan, N., Podobnik, A., Turk, B., Vreš, B., Ravnik, V., Frajman, B., Strgulc Krajšek, S., Trčak, B., Bačič, T., Fischer, M. A., Eler, K. \& Surina, B. 2007: Mala flora Slovenije. Ključ za določanje praprotnic in semenk. Četrta, dopolnjena in spremenjena izdaja. Tehniška založba Slovenije, Ljubljana, $967 \mathrm{pp}$.

Mertz, P. 2000: Pflanzengesellschaften Mitteleuropas und der Alpen. Erkennen-Bestimmen. -Bewerten. Ecomed, Landsberg/Lech, 511 pp.

Oksanen, J., Blanchet, F. G., Kindt, R., Legendre, P., Minchin, P. R., O'Hara, R. B., Simpson, G. L., Solymos, P., Stevens, M. H. \& Wagner, H. 2012: vegan: Community Ecology Package. R package version 2.0-4. http://CRAN.R-project. org/package=vegan

Paulin, A. 1915: Über einige für Krain neue oder seltene Pflanzen und die Formationen ihrer Standorte I. Carniola 6: 117-125, 186-209.

Petkovšek, V. 1945: Na savinjskih ognjenikih. Planinski zbornik 1945. Planinsko društvo Slovenije, Ljubljana: 194-203.

Pisetta, M., Montecchio, L., Longa, C. M. O., Salvadori, C., Zottele, F. \& Maresi, G. 2012: Green alder decline in the Italian Alps. Forest Ecology and Management 281: 75-83.

Piskernik, M., 1982: Bioekološka in sestojna predstavitev mikroreliefnih gozdnih združb slovenskega ozemlja. Fitocenološke razpredelnice. Inštitut za gozdno in lesno gospodarstvo. Strokovna in znanstvena dela 75: 1-149.

Podani, J., 2001: SYN-TAX 2000. Computer Programs for Data Analysis in Ecology and Systematics. User's Manual, Budapest, 53 pp.

R Development Core Team 2012: R: A language and environment for statistical computing. $\mathrm{R}$ Foundation for Statistical Computing, Vienna, Austria. ISBN 3-900051-07-0, URL http:// www.R-project.org/.

Seliškar, T., Vreš, B. \& Seliškar, A. 2003: FloVegSi 2.0. Fauna, Flora, Vegetation and Paleovegetation of Slovenia. Computer programme for arranging and analysis of biological data. Biološki inštitut ZRC SAZU, Ljubljana. 
Seljak, G. 1974: Travniška vegetacija Porezna. Diplomsko delo. Univerza v Ljubljani, Biotehniška fakulteta, Oddelek za agronomijo, Ljubljana, 103 pp.

Sørensen, Th. 1948: A method of establishing groups of equal amplitude in plant sociology based on similarity of species content. Det Kongelige Danske Videnskaberns Selskab, Biologiske Skrifter (København) 5 (4): 1-34.

Stefanović, V. 1986: Fitocenologija sa pregledom šumskih fitocenoza Jugoslavije. 2. izdanje. Svijetlost, Sarajevo, 269 pp.

Stefanović, V. \& Beus, V. 1982: Die Gesellschaft der Grünerle (Athyrio-Alnetum viridis ass. nova) auf dem Gebirge Vranica in Zentralbosnien. Glasnik Zemaljskog muzeja Bosne i Hercegovine u Sarajevu. Prirodne nauke. Nova serija, Sveska 19-20: 55-64.

Steiger, P., 1994: Wälder der Schweiz. Von Lindengrün zum Lärchengold. Vielfalt der Waldbilder und Waldgesellschaften in der Schweiz. Thun - Ott, 361 pp.

Steiger, P. 2010: Wälder der Schweiz. Von Lindengrün zum Lärchengold. Vielfalt der Waldbilder und Waldgesellschaften in der Schweiz. 4. Auflage. Ott Verlag Bern.

Suppan, U., Prügger, J. \& Mayrhofer, H. 2000: Catalogue of the lichenized and lichenicolous fungi of Slovenia. Bibliotheca Lichenologica 76: $1-215$.

Surina, B. 2005: Subalpinska in alpinska vegetacija Krnskega pogorja v Julijskih Alpah. Scopolia (Ljubljana) 57: 1-122.

Surina, B. \& Rakaj, M. 2007: Subalpine beech forests with Hairy Alpenrose (Polysticho lonchitis-Fagetum rhododendretosum hirsuti subass. nova) on Mt. Snežnik (Liburnian Karst, Dinaric Mts). Hacquetia 6 (2): 195-208.

Šercelj, A. 1996: The origins and development of forests in Slovenia. Slovenska akademija znanosti in umetnosti. Razred za naravoslovne vede, Dela (Opera) 35: 1-142, Ljubljana.

Šilc, U. \& Čarni, A. 2012: Conspectus of vegetation syntaxa in Slovenia. Hacquetia 11 (1): 113-164.

Urbančič, M., Simončič, P., Prus, T. \& Kutnar, L. 2005: Atlas gozdnih tal. Zveza gozdarskih društev Slovenije, Gozdarski vestnik \& Gozdarski inštitut Slovenije, Ljubljana. 100 pp.

Weber, H. E., Moravec, J. \& Theurillat, J. P. 2000: International Code of Phytosociological Nomenclature. 3rd. Edition. Journal of Vegetation Science 11 (5): 739-766.
Willner, W. 2007: Fagion sylvaticae Luquet 1926. In: Willner, W. \& G. Grabherr (eds.): Die Wälder und Gebüsche Österreichs. Ein Bestimmungswerk mit Tabellen. 1. Textband. Spektrum Akademischer Verlag in Elsevier, Heidelberg, pp. 144-164.

Wraber, M. 1960: Fitocenološka razčlenitev gozdne vegetacije v Sloveniji. Zbornik ob 150. letnici botaničnega vrta v Ljubljani, Ljubljana, pp. 49-94.

Talbot, S. S., Talbot, S. L. \& Daniëls, F. J. A. 2005: Comparative phytosociological investigation of subalpine alder thickets in southwestern Alaska and the North Pacific. Phytocoenologia 35 (4): 727-759.

Theurillat, J.-P. 2004: Pflanzensoziologisches System. In: Aeschimann, D., K. Lauber, D. M. Moser \& J.-P. Theurillat: Flora alpina 3: Register. Haupt Verlag, Bern, Stuttgart, Wien, pp. 301-313.

Tzonev, R, Dimitrov, M. \& Roussakova, V. 2009: Syntaxa according to the Braun-Blanquet approach in Bulgaria. Phytologia Balcanica 15 (2): 209-233.

Zupančič, M. 1999: Smrekovi gozdovi Slovenije (Spruce forests in Slovenia). Dela 4. raz. SAZU 36, Ljubljana, 212 s. + tabele.

Zupančič, M. 2012: Syntaxonomic problems of altimontane beech forests of the alliance Aremonio-Fagion in Slovenia. Folia biologica et geologica 53 (1-2): 83-127.

Zupančič, M. 2013: New considerations on southeast-Alpine and Dinaric-central Balkan dwarf pine. Hrvatska misao 17 (1) / 13 (61) nova serija sv. 46: 156-172.

Zupančič, M. \& Žagar, V. 2001: Asociacija Salicetum waldsteinianae Beger $1922 \mathrm{v}$ jugovzhodnih apneniških Alpah (Slovenija). Razprave 4. razreda SAZU (Ljubljana) 42-2: 275-310.

Zupančič, M., Žagar, V., Culiberg, M. 2006: Slovene Pinus mugo scrub in comparison with $\mathrm{Eu}-$ ropean Pinus mugo scrub (Rhodothamno-Rhododendretum hirsuti var. geogr. Paederota lutea). Slovenska akademija znanosti in umetnosti, razred za naravoslovne vede, Dela 40: 1-112 pp. + dve fitocenološki tabeli (two phytosociological tables), Ljubljana.

Received 21. 3. 2013

Revision received 13. 9. 2013

Accepted 17. 9. 2013 


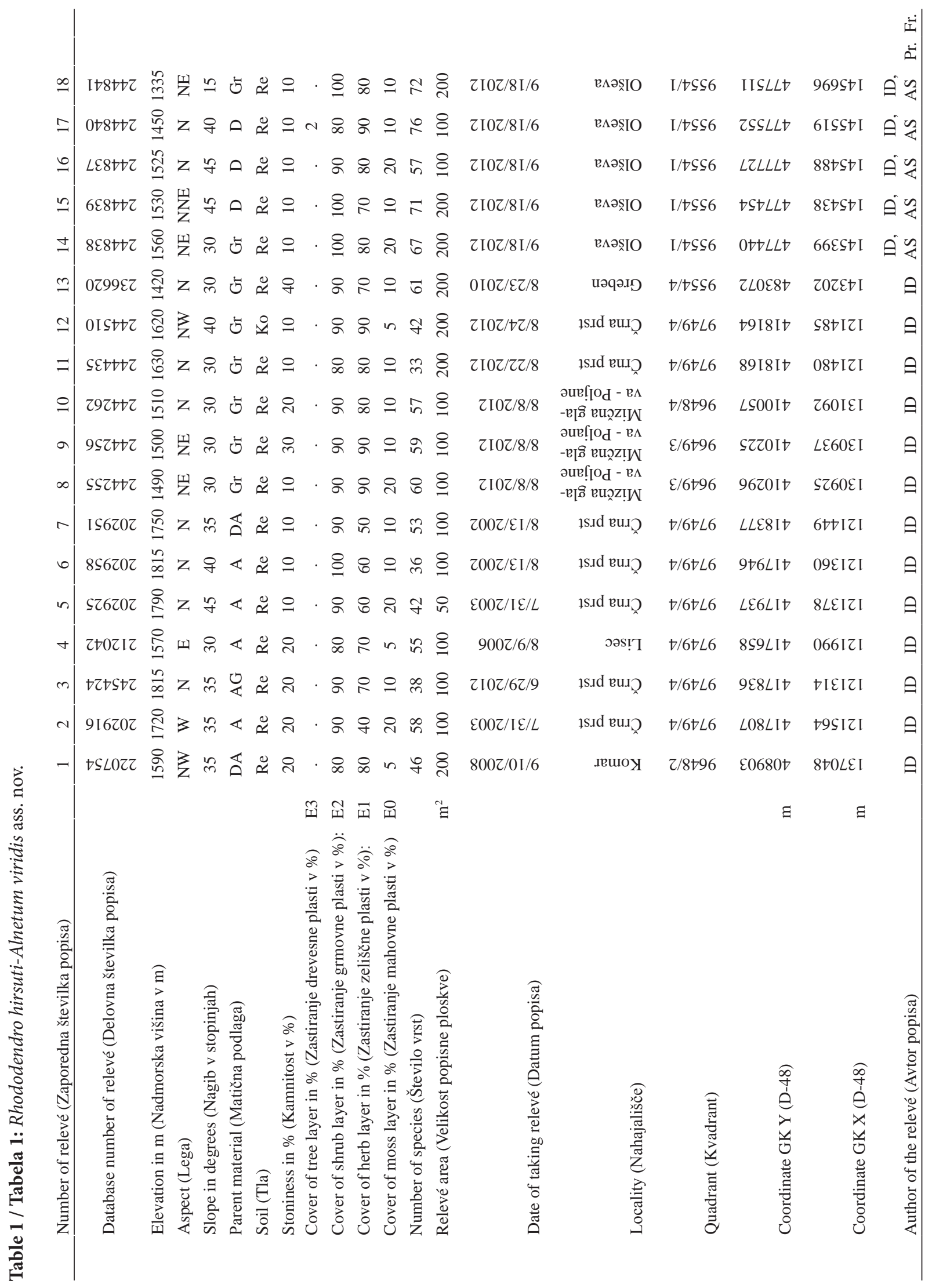




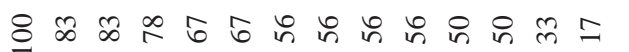

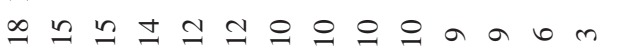
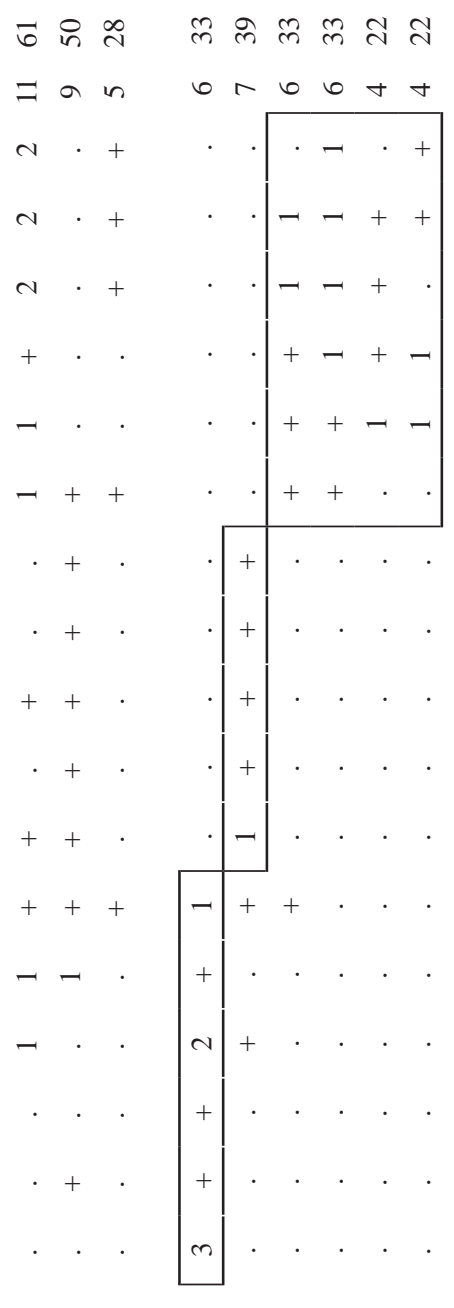

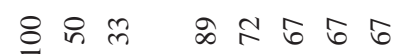
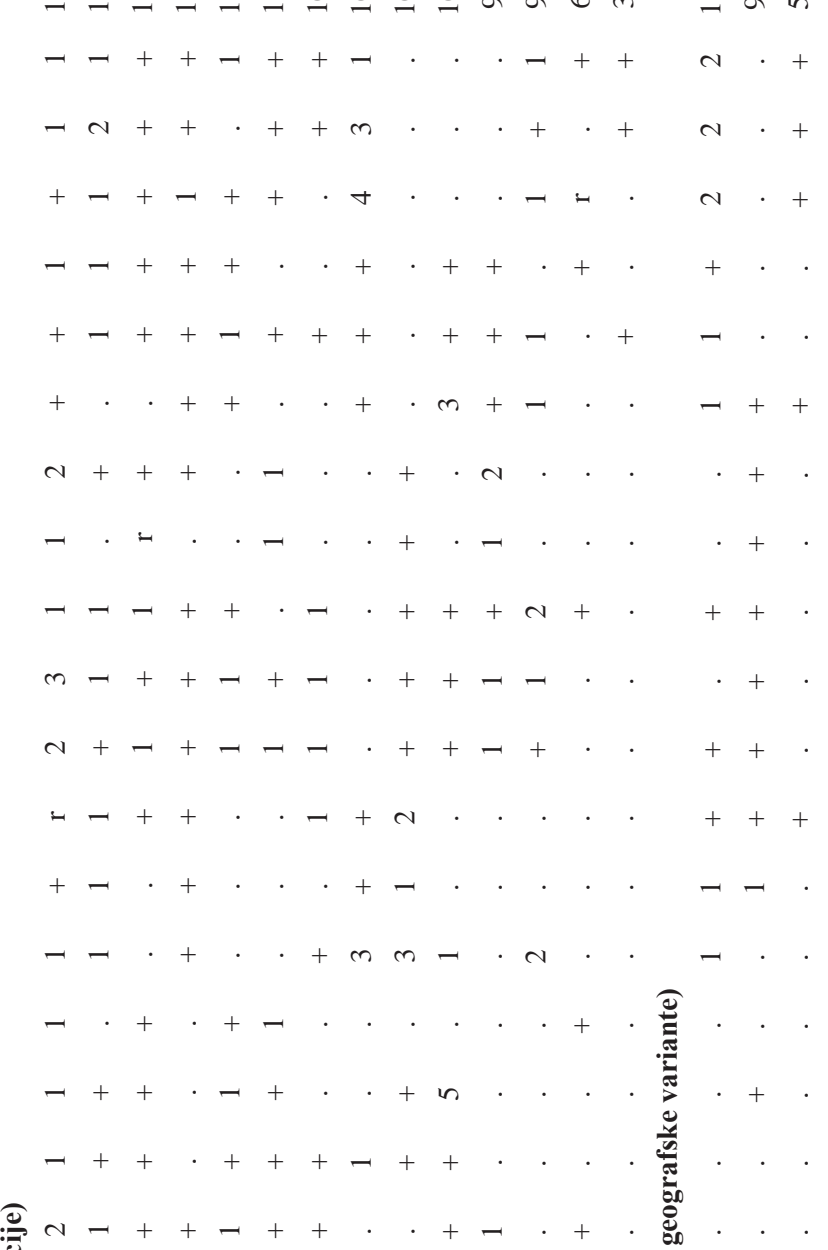

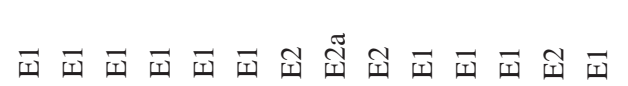

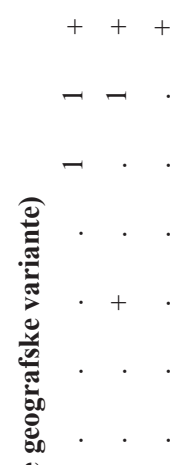

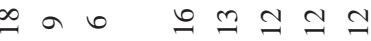

$\neg \sim \cdot++r$

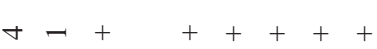

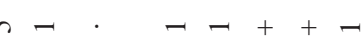

เ $\neg . \quad-\neg-7$

เ

n. $.46-4$

เ . . $\rightarrow-1$

$\neg+. \rightarrow \sim \sim+$

$\omega+\cdot-\pi \cdot n$

เ $\cdot \pi-+2$

เ $\cdot$ N

เ. N . .

$+\cdot \cdot$

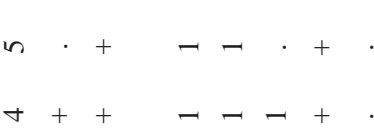

$-7-$

을되되되되되도

तิ तิ

되되되되 되

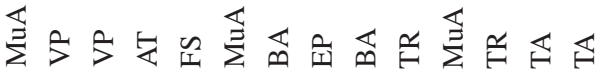

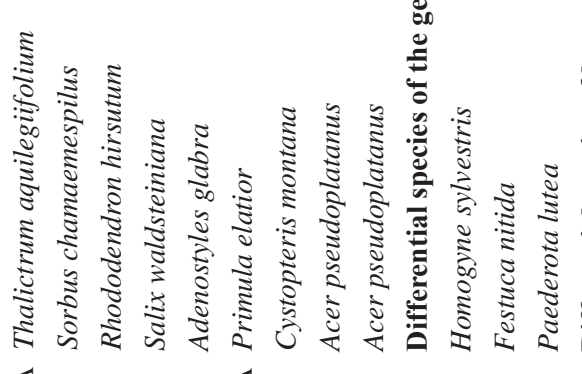

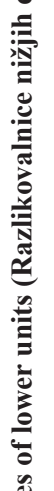

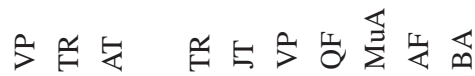

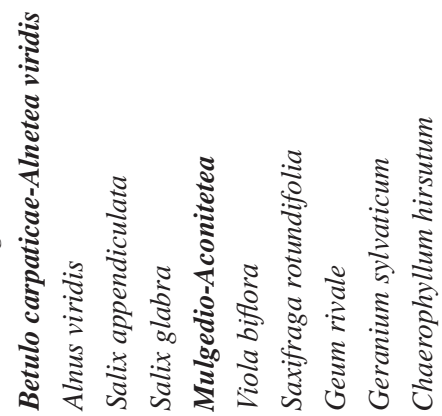

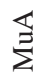




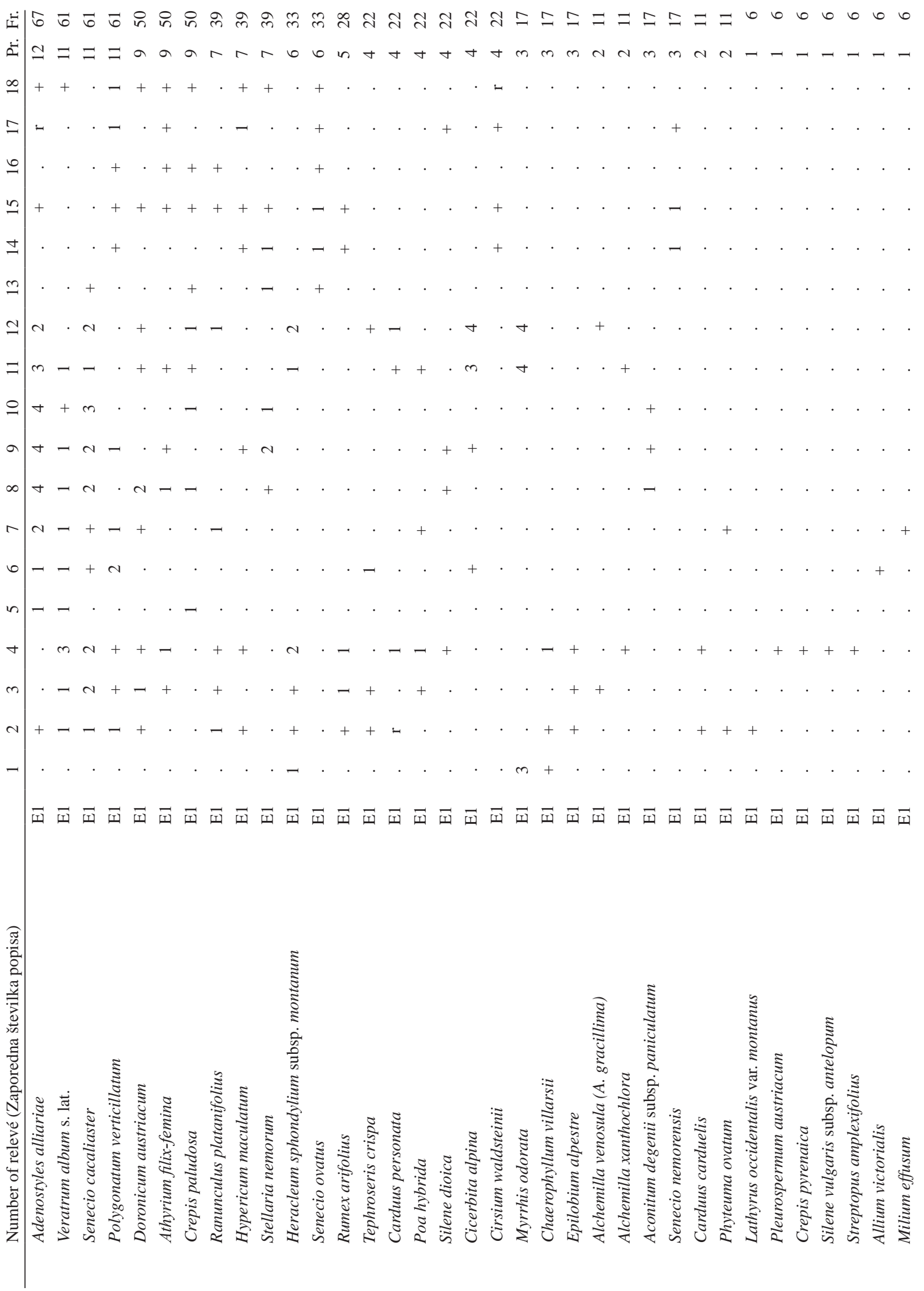


0 0 0 ต

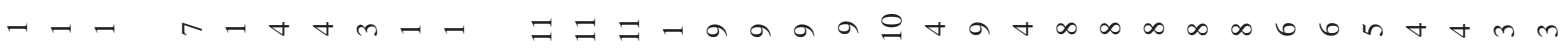

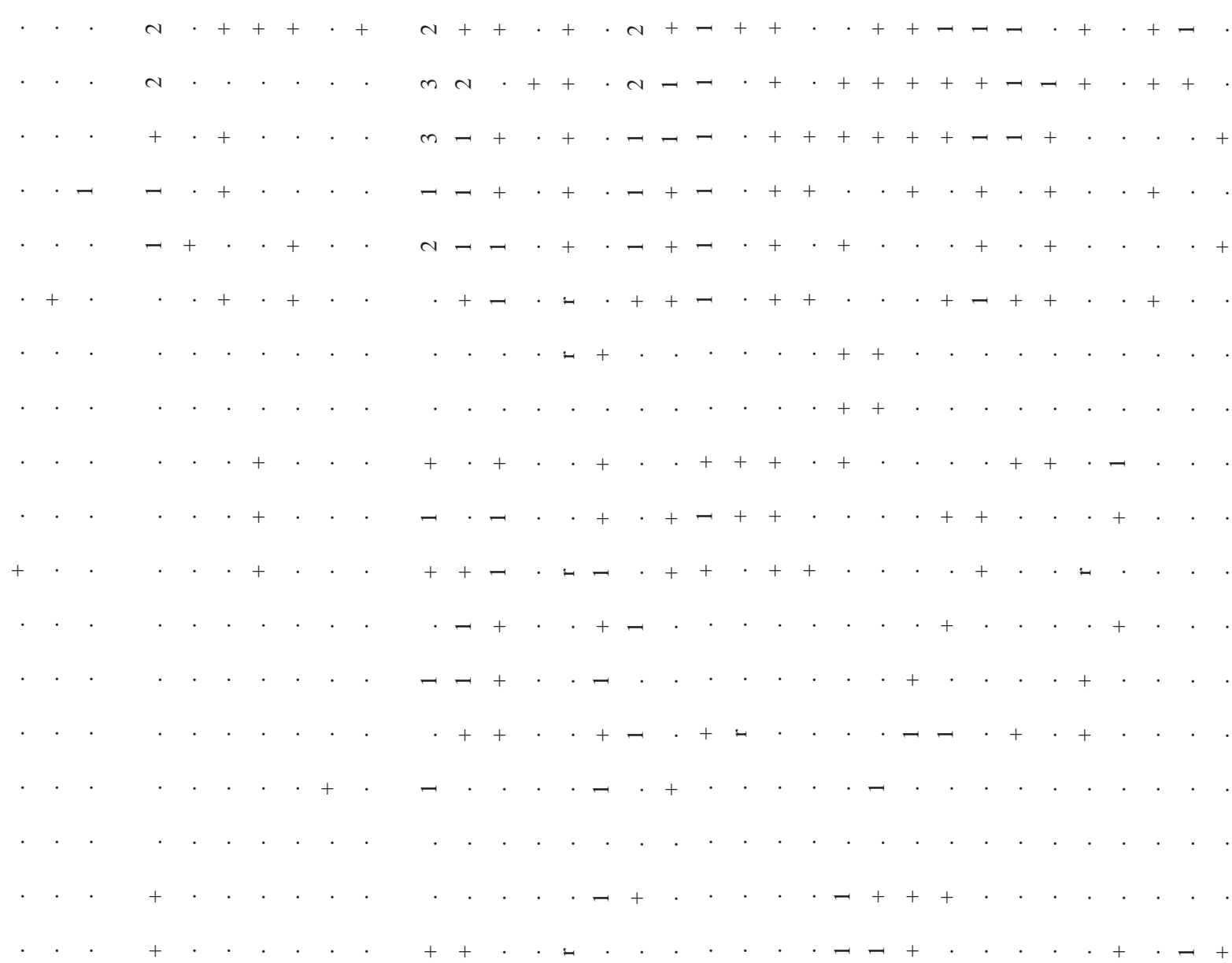

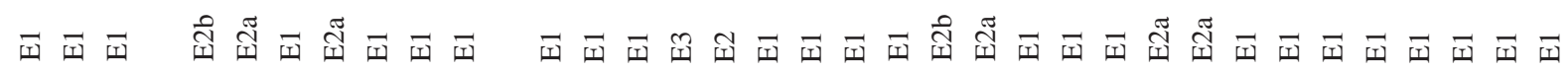

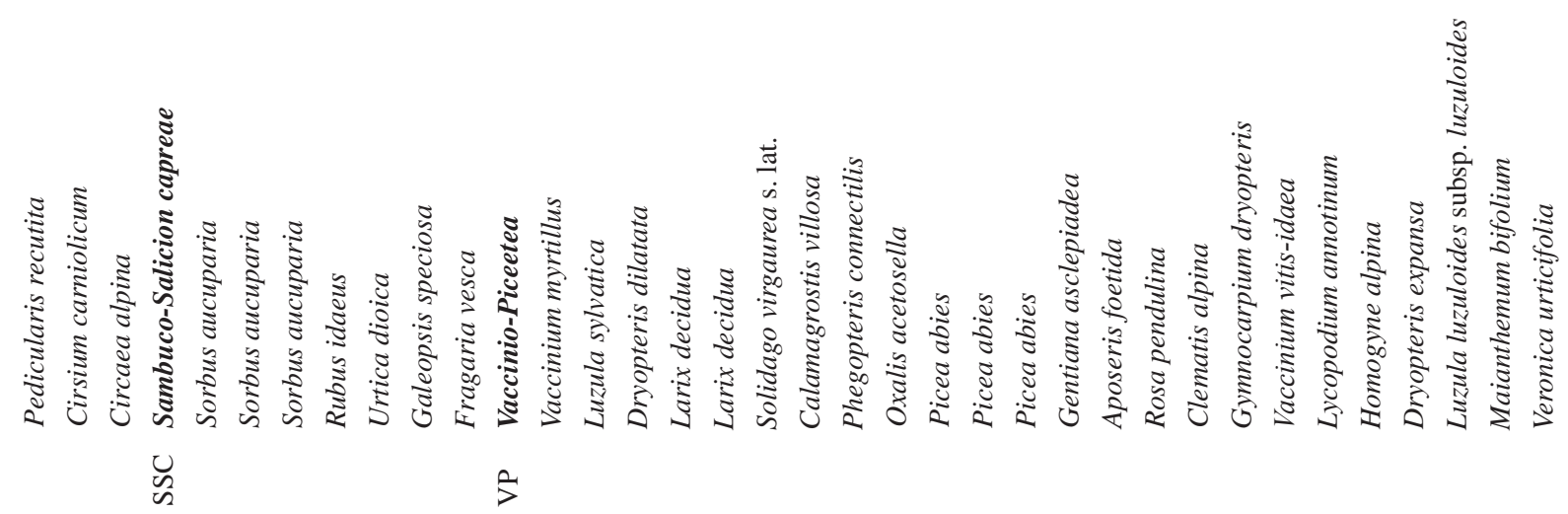




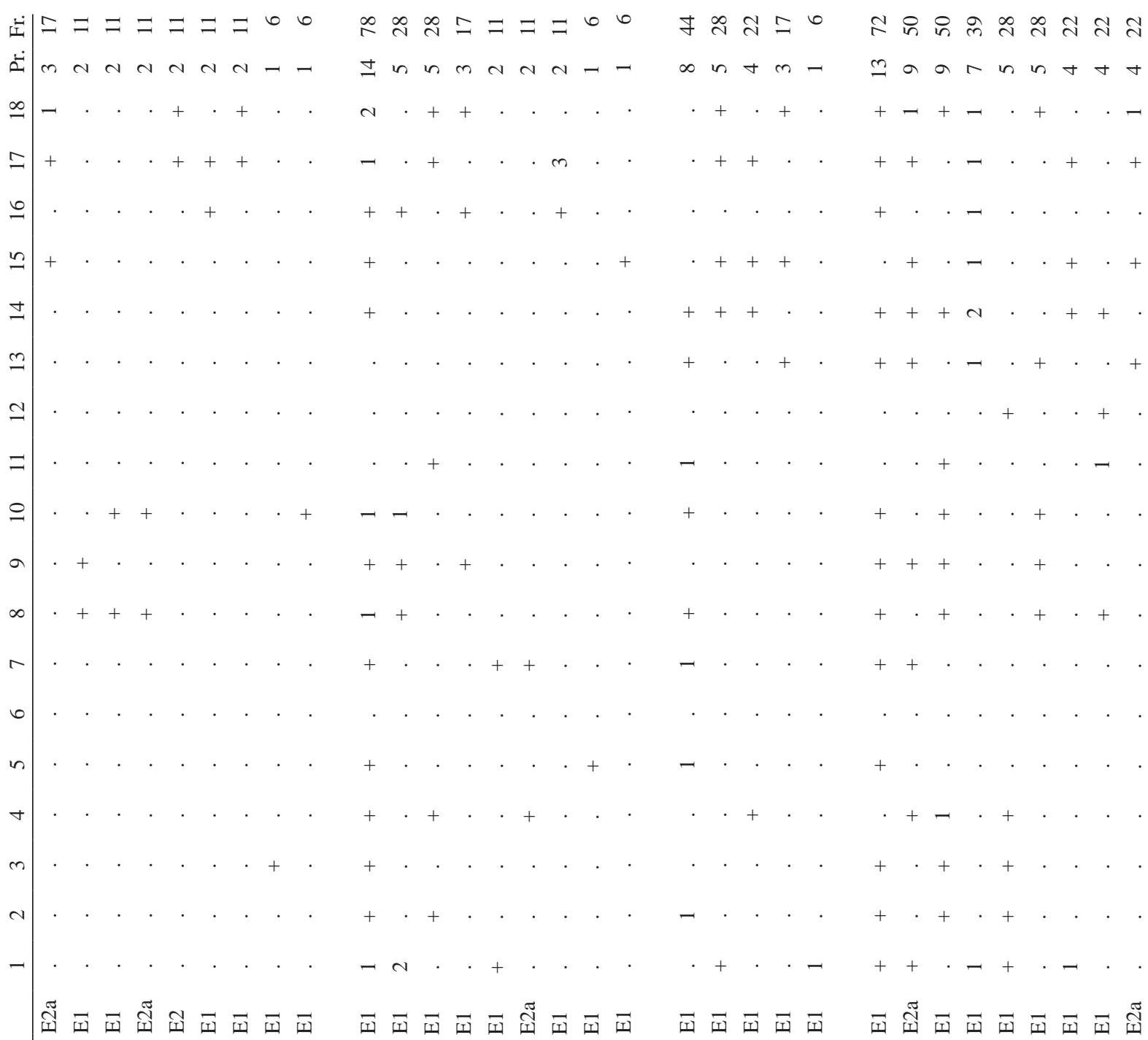




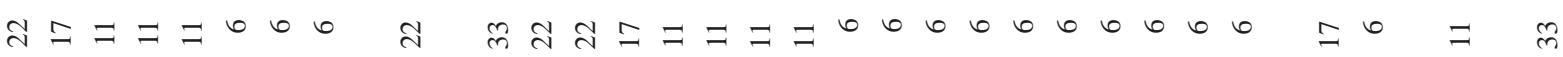

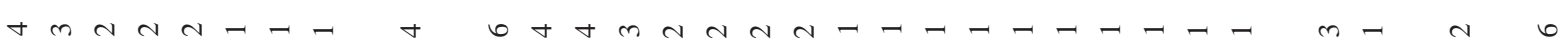

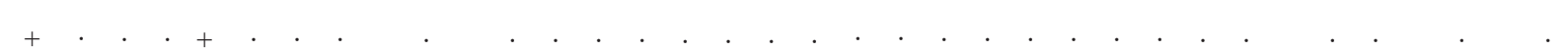

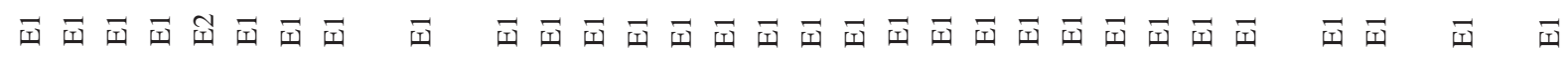

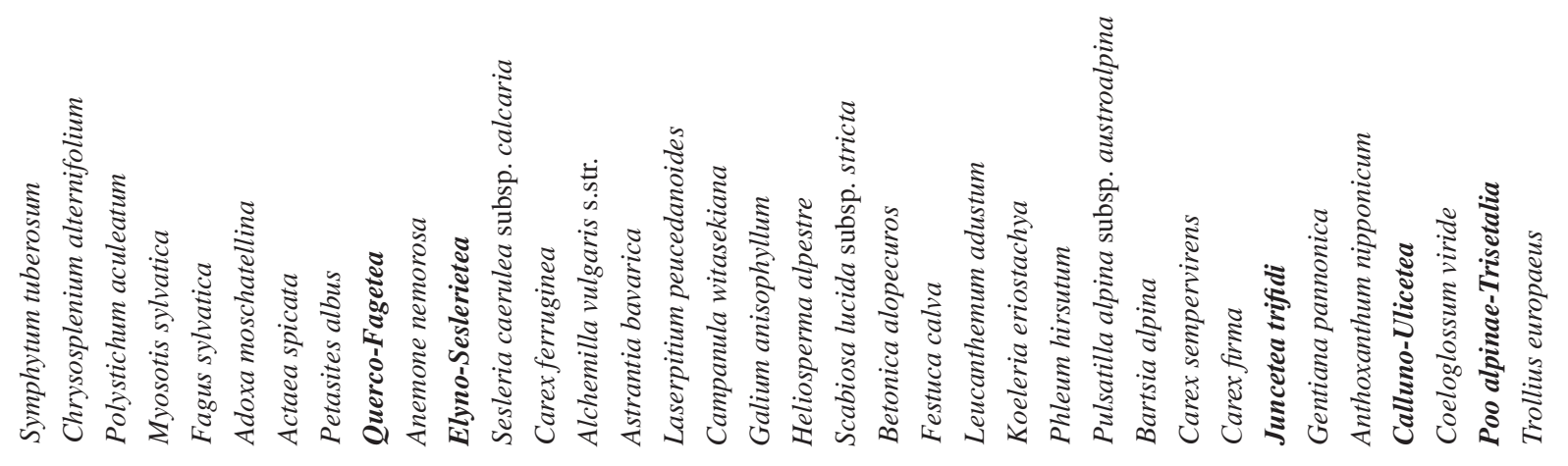

岂 되

占包嵒 


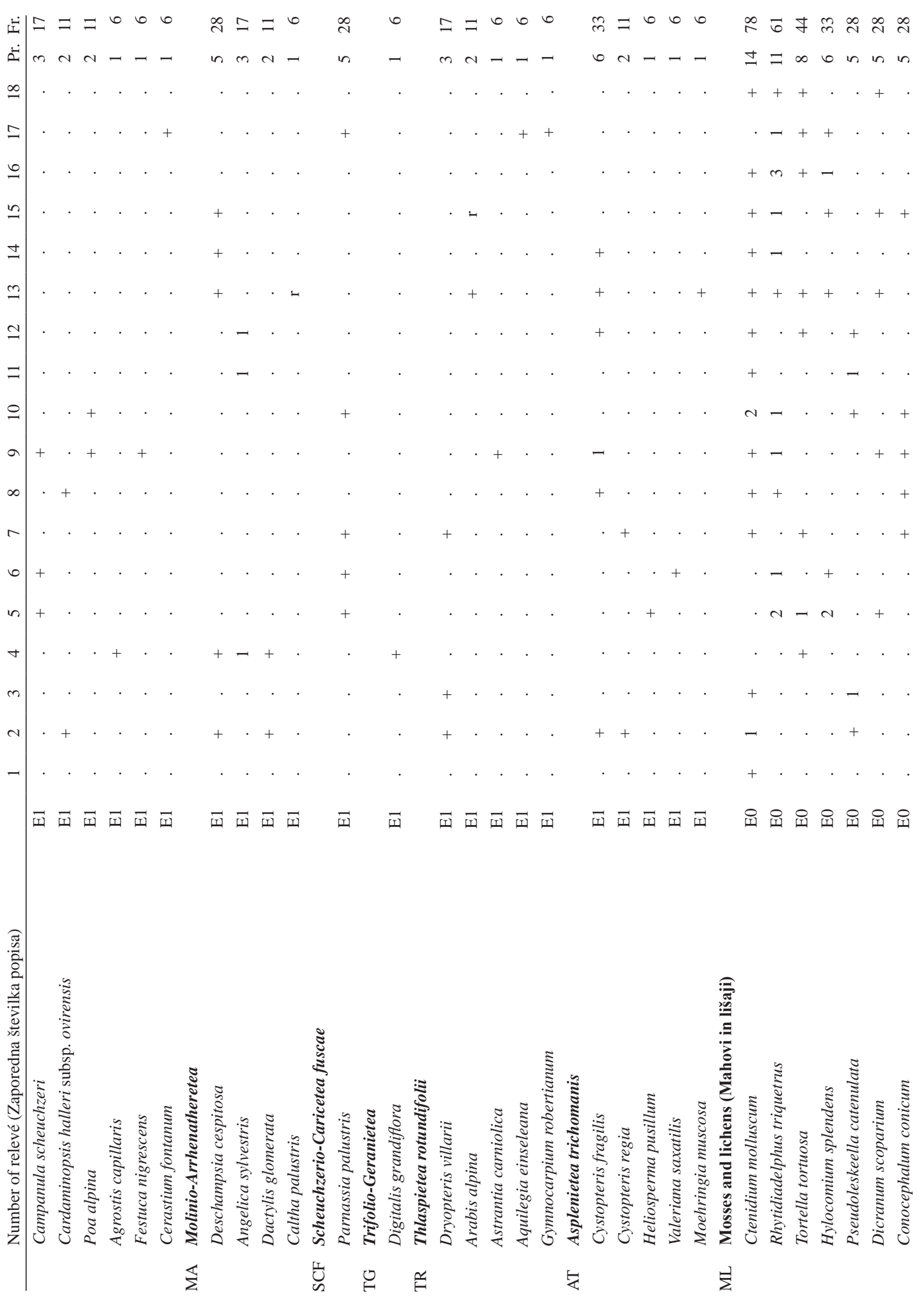


สิ

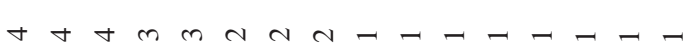

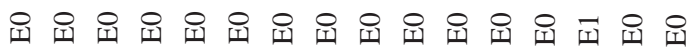

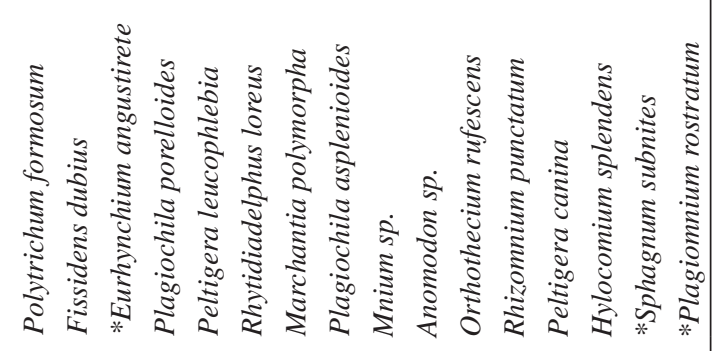

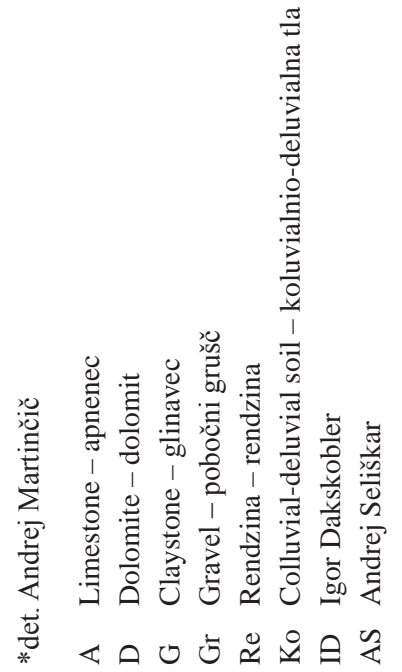




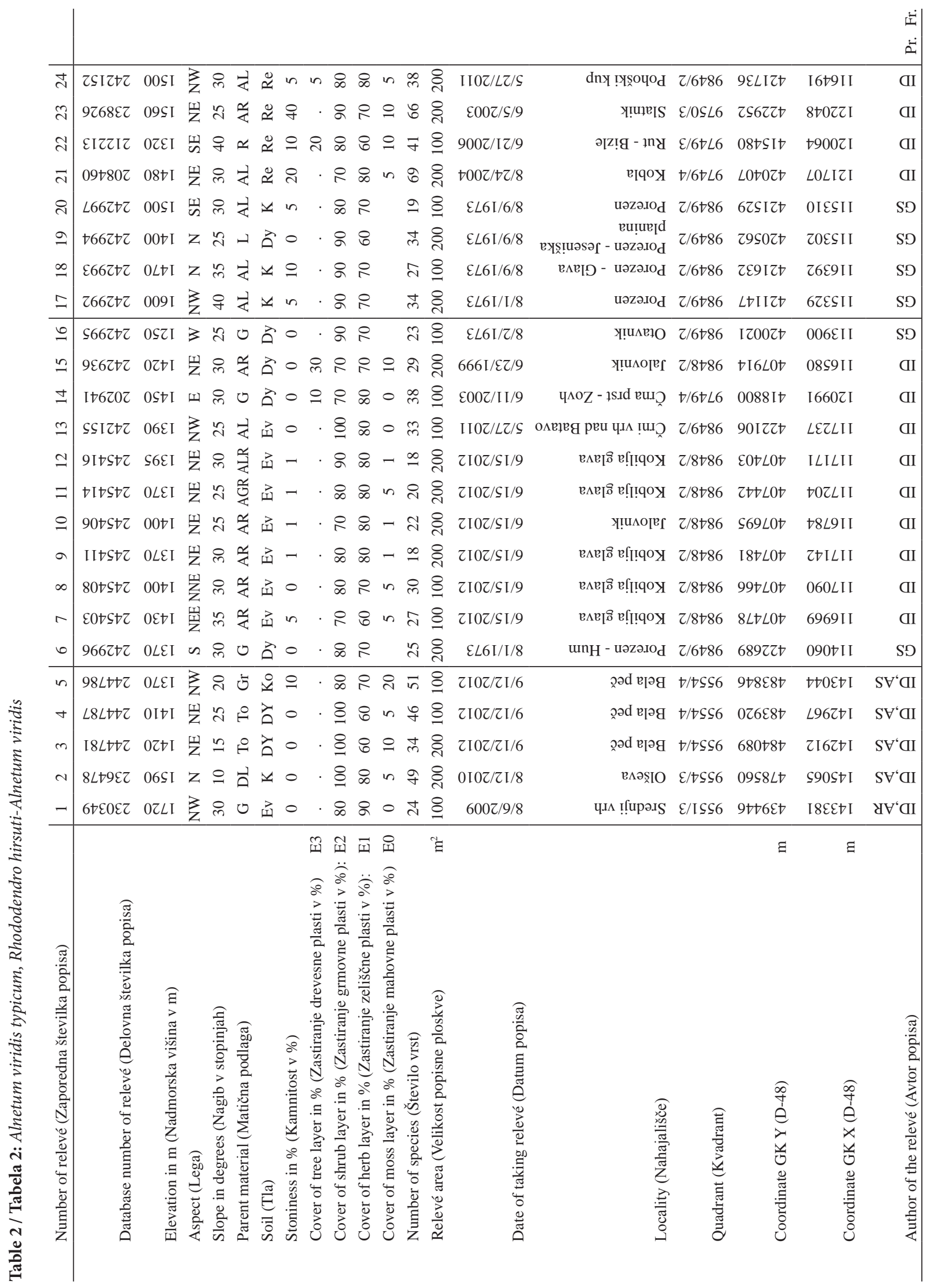


అ $m 0+\wedge 00$ ก

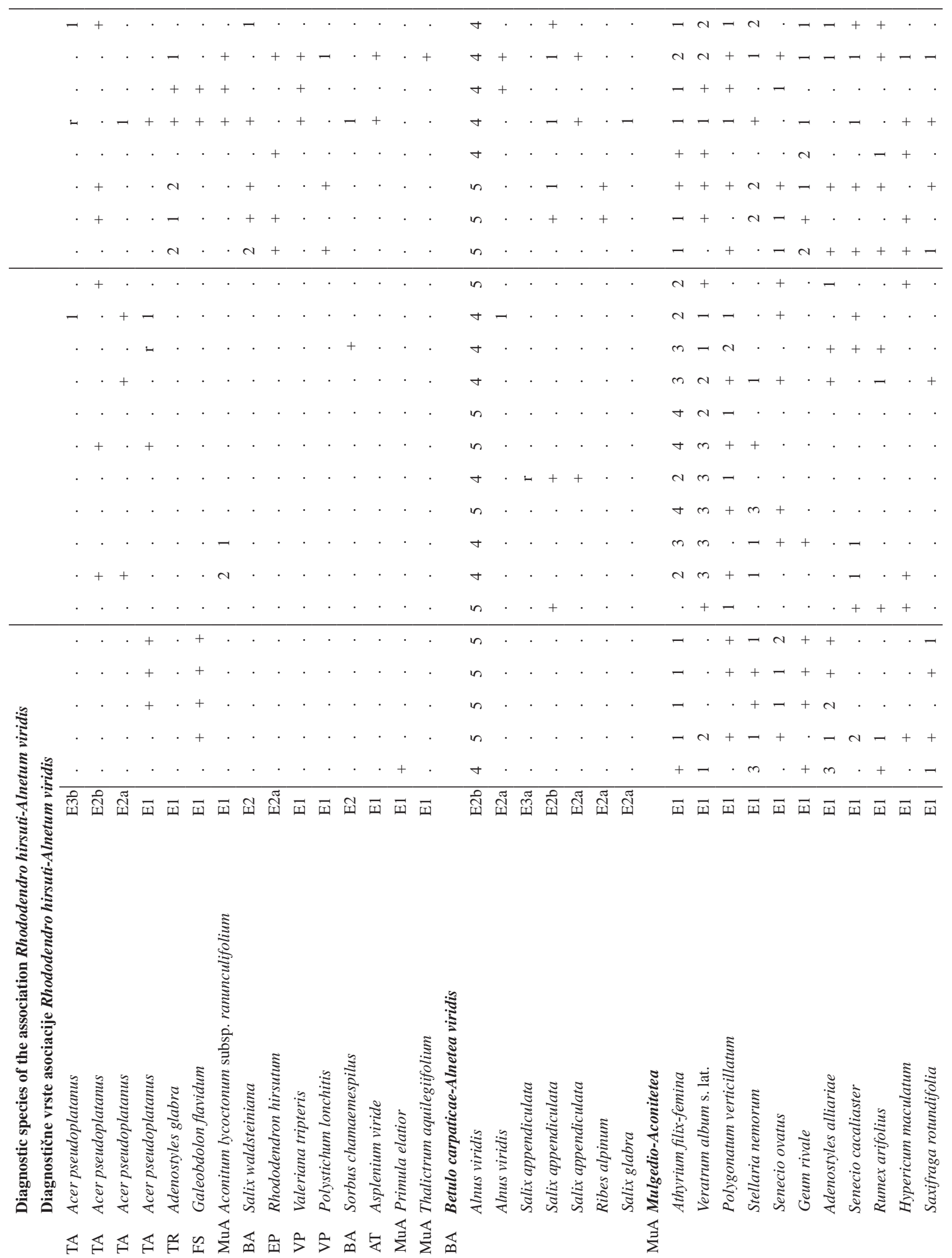




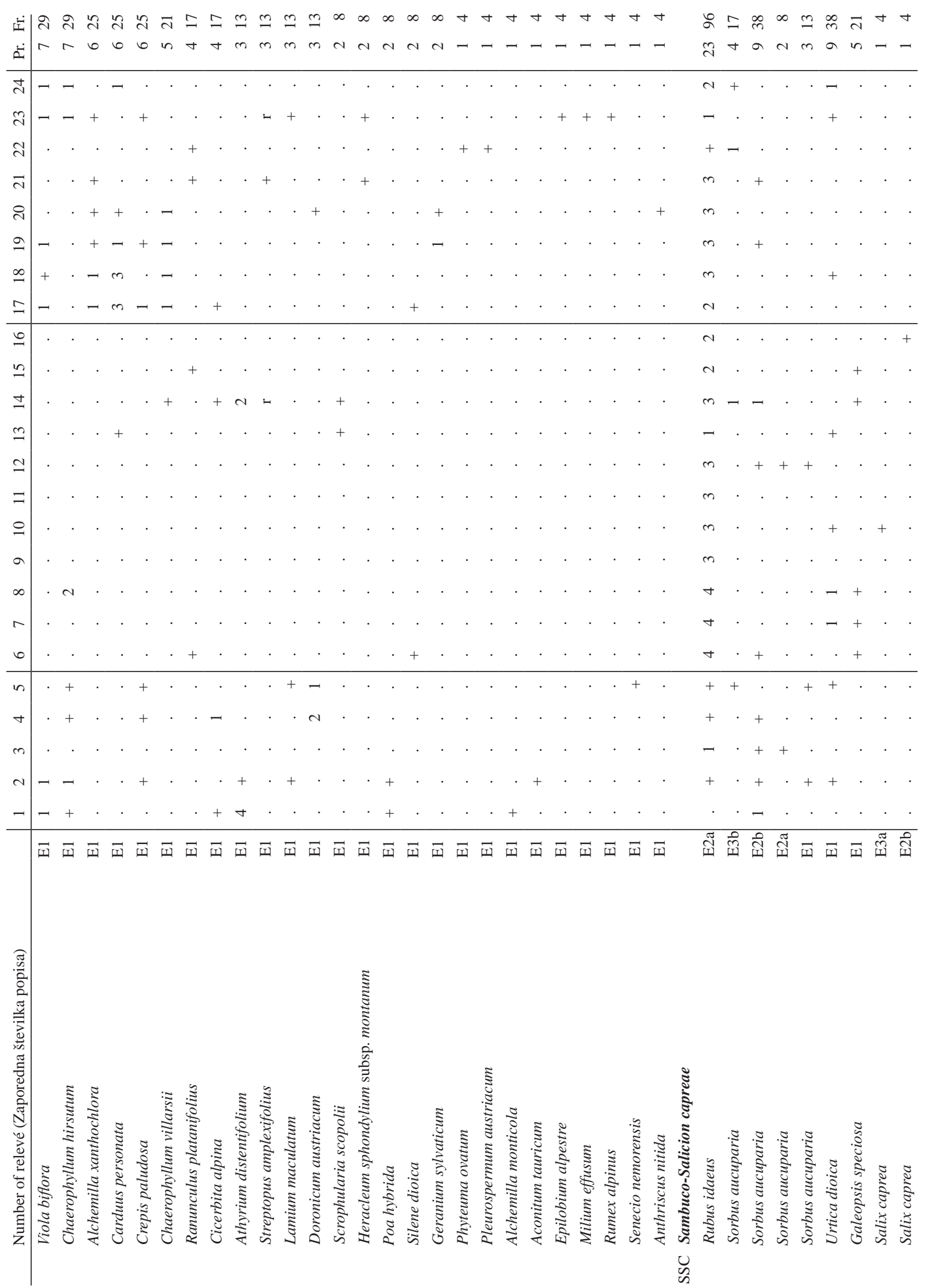


$\infty$ న

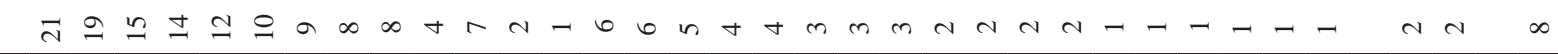
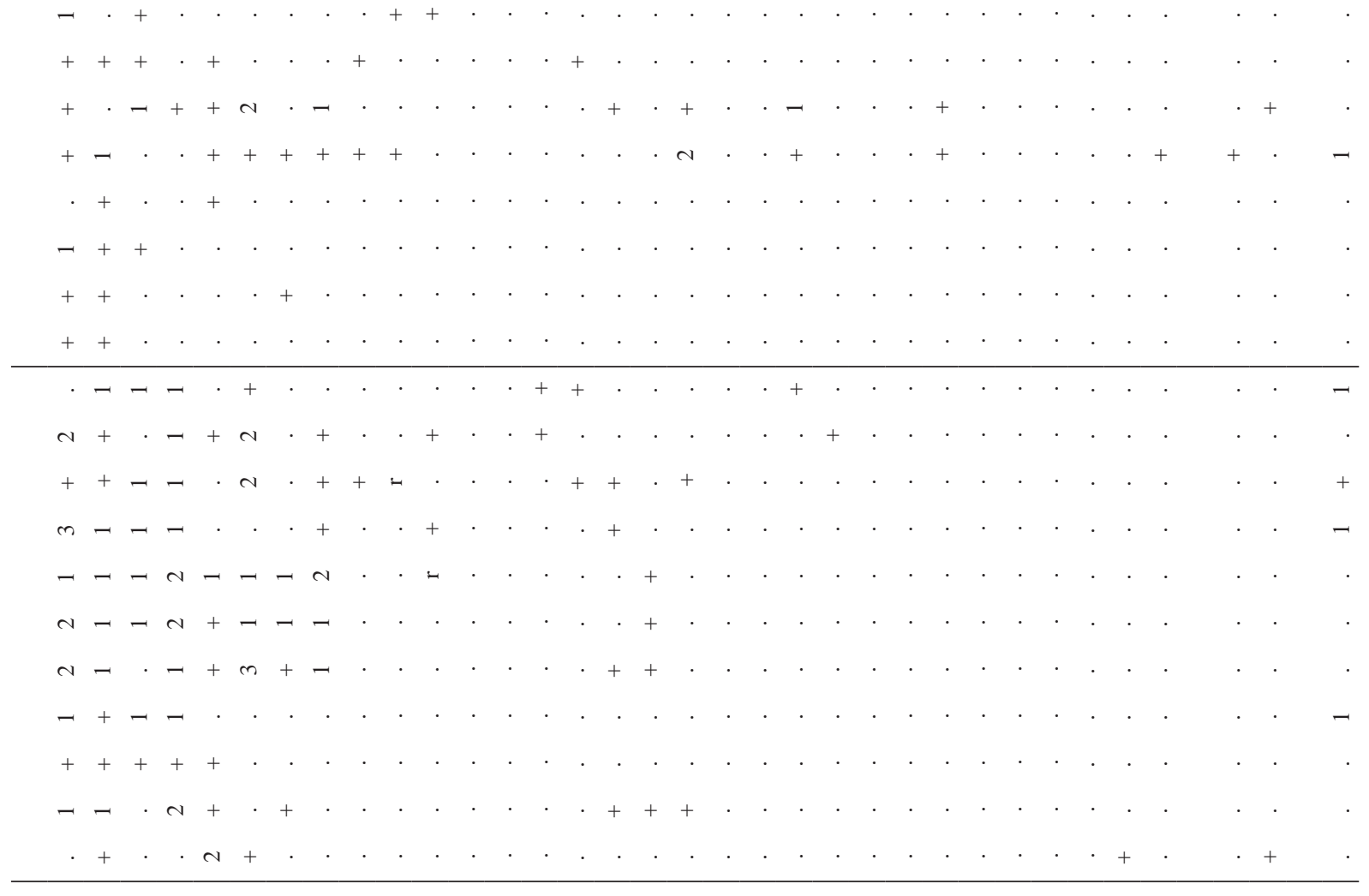

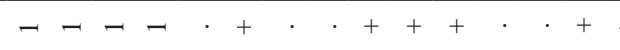

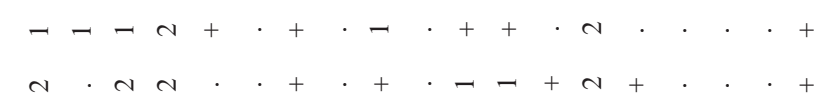

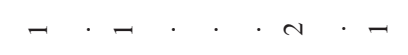

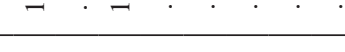

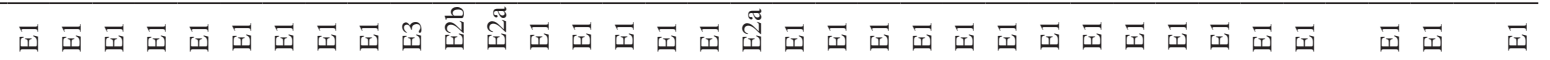

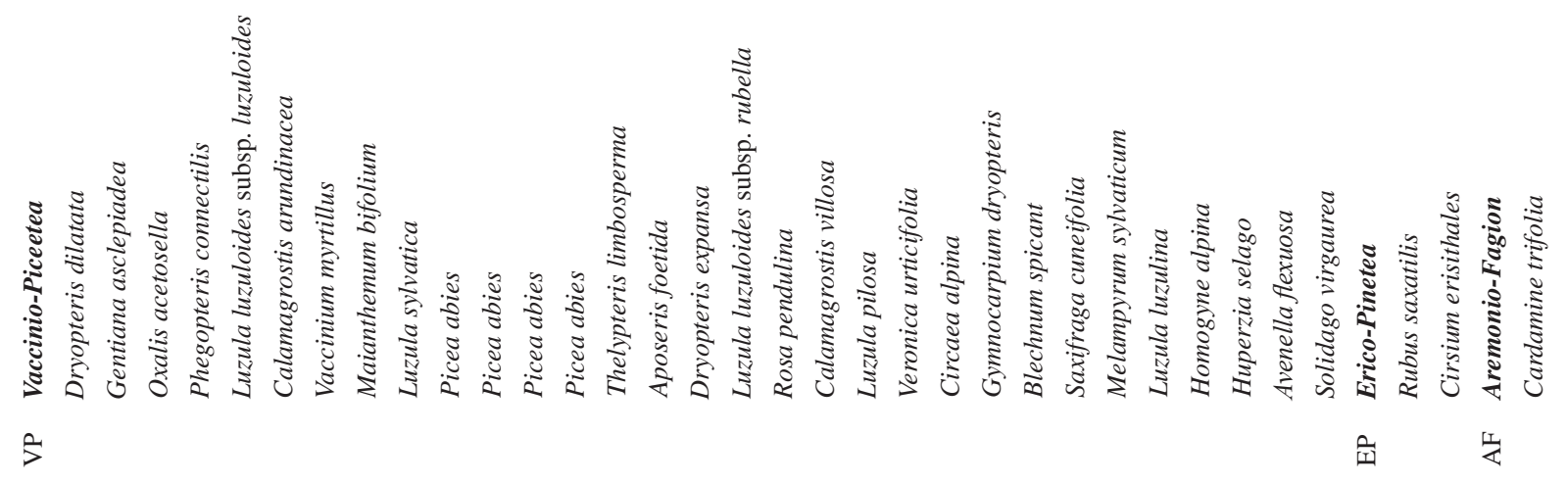




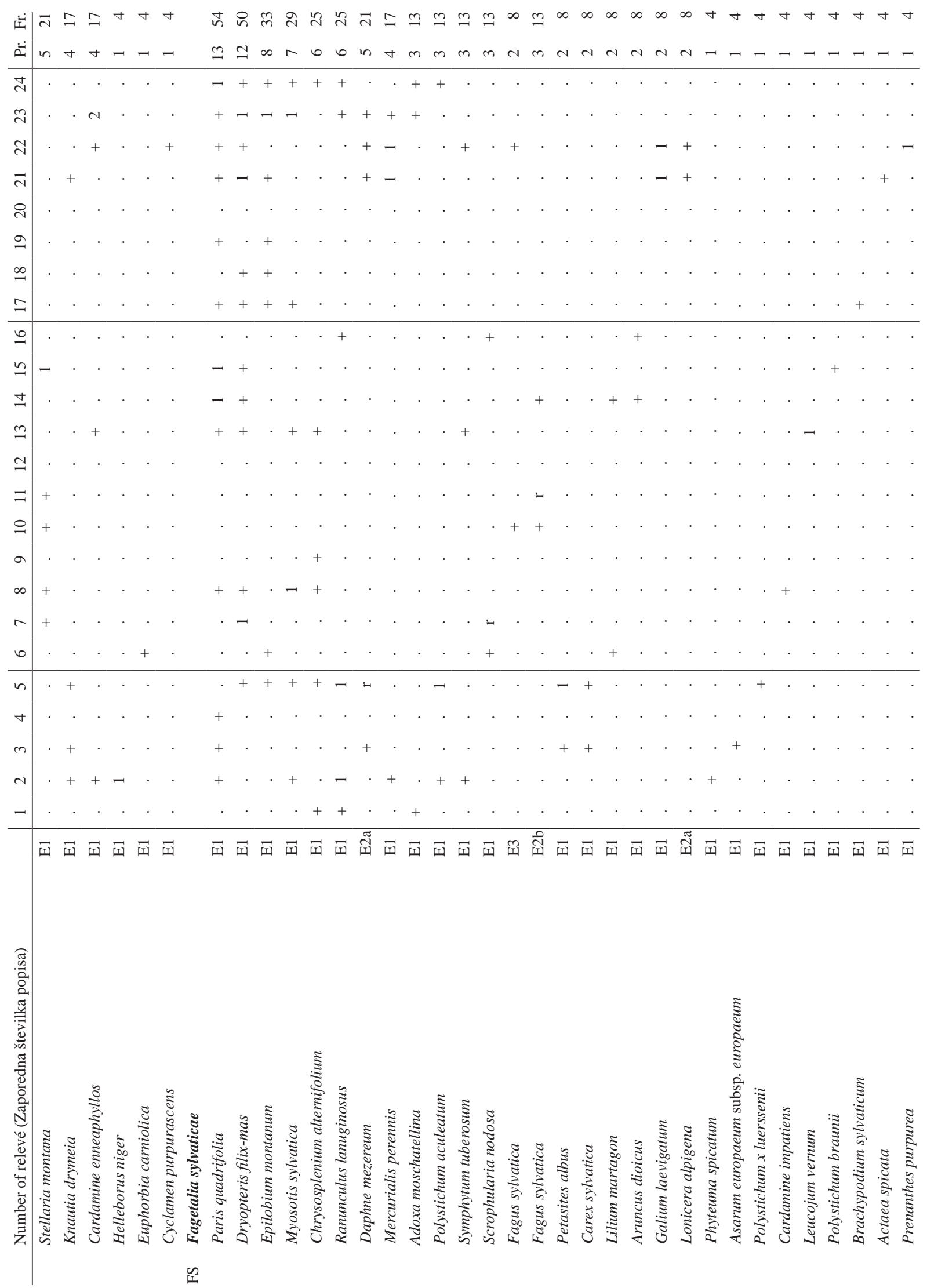



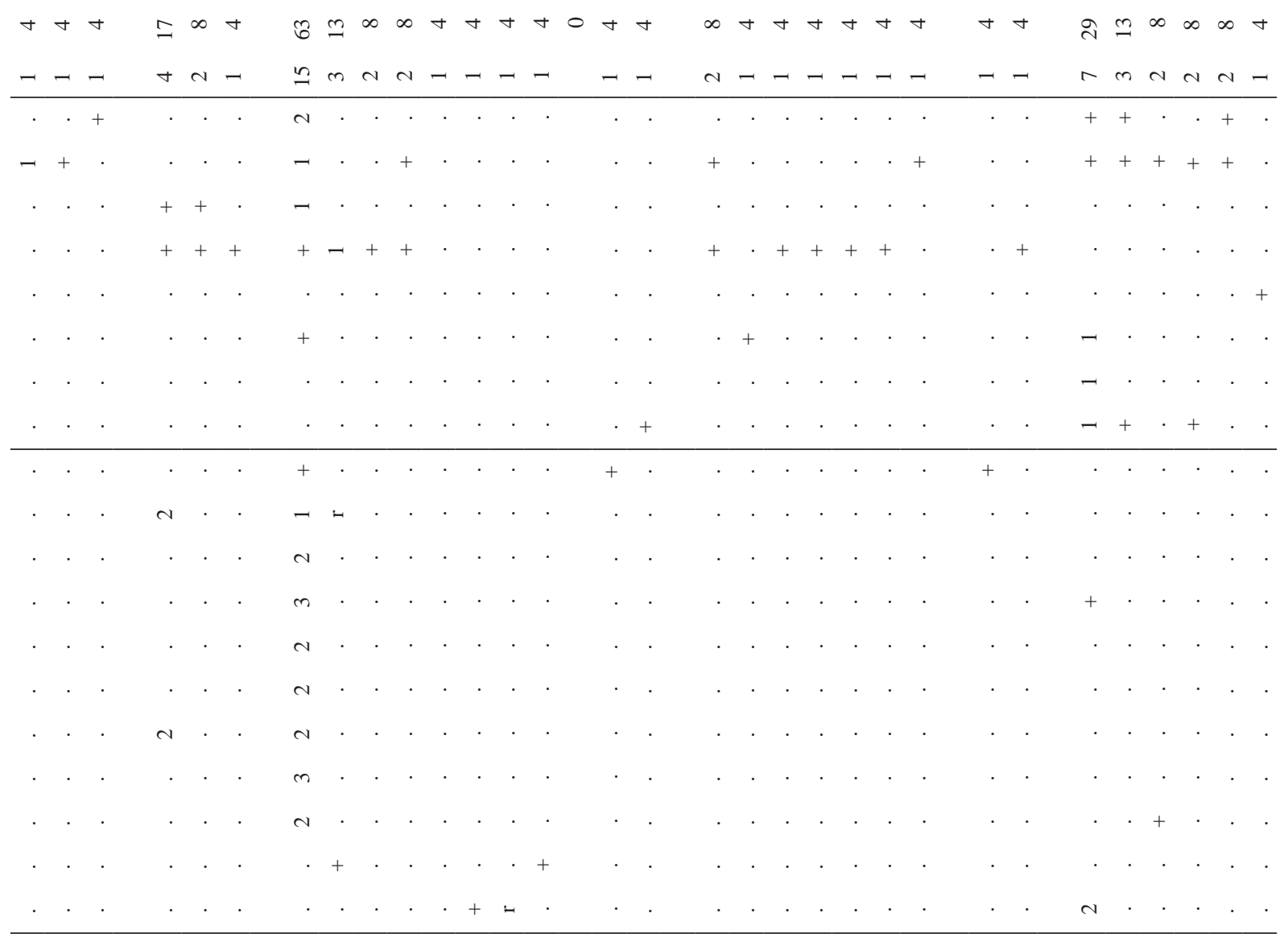

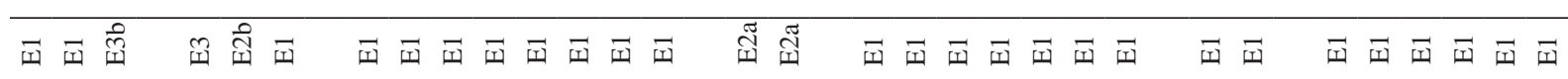

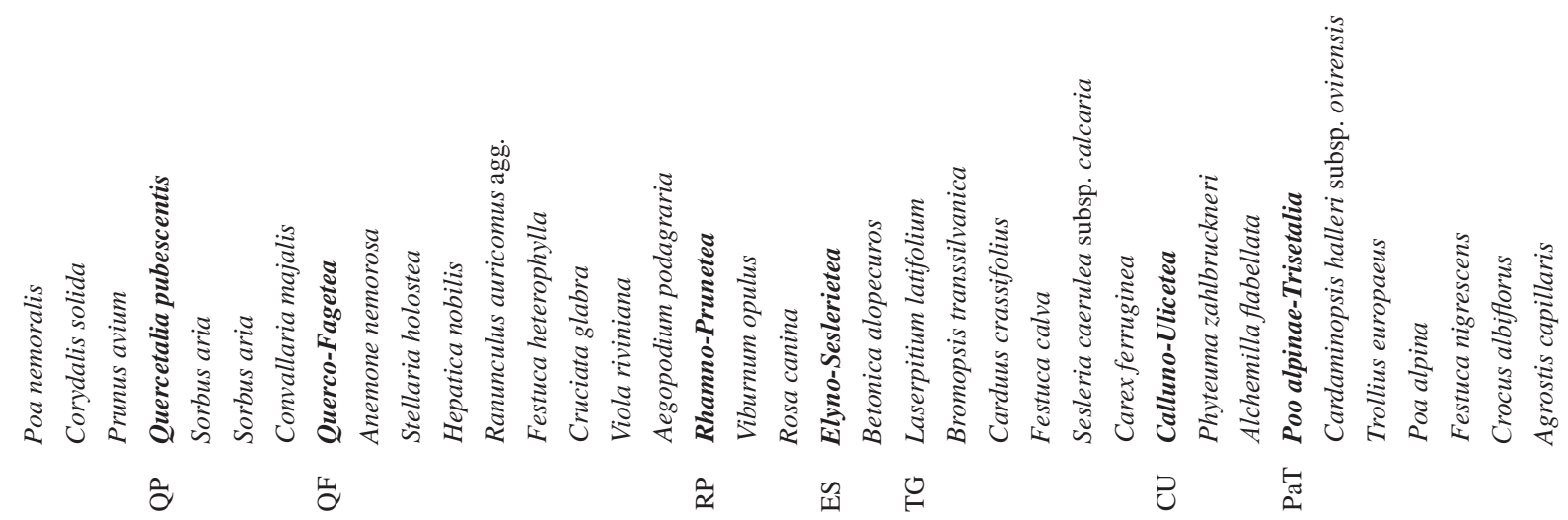




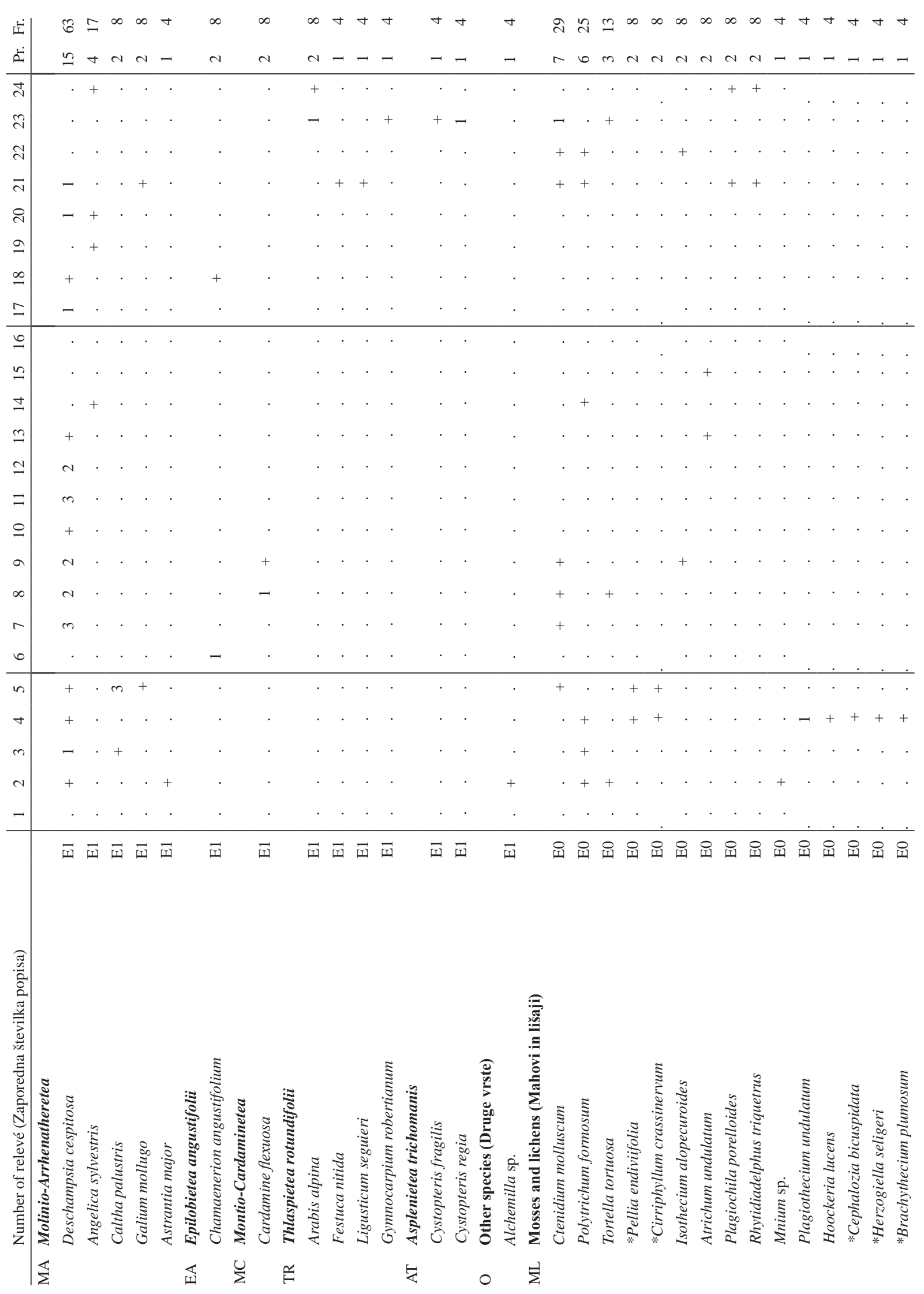




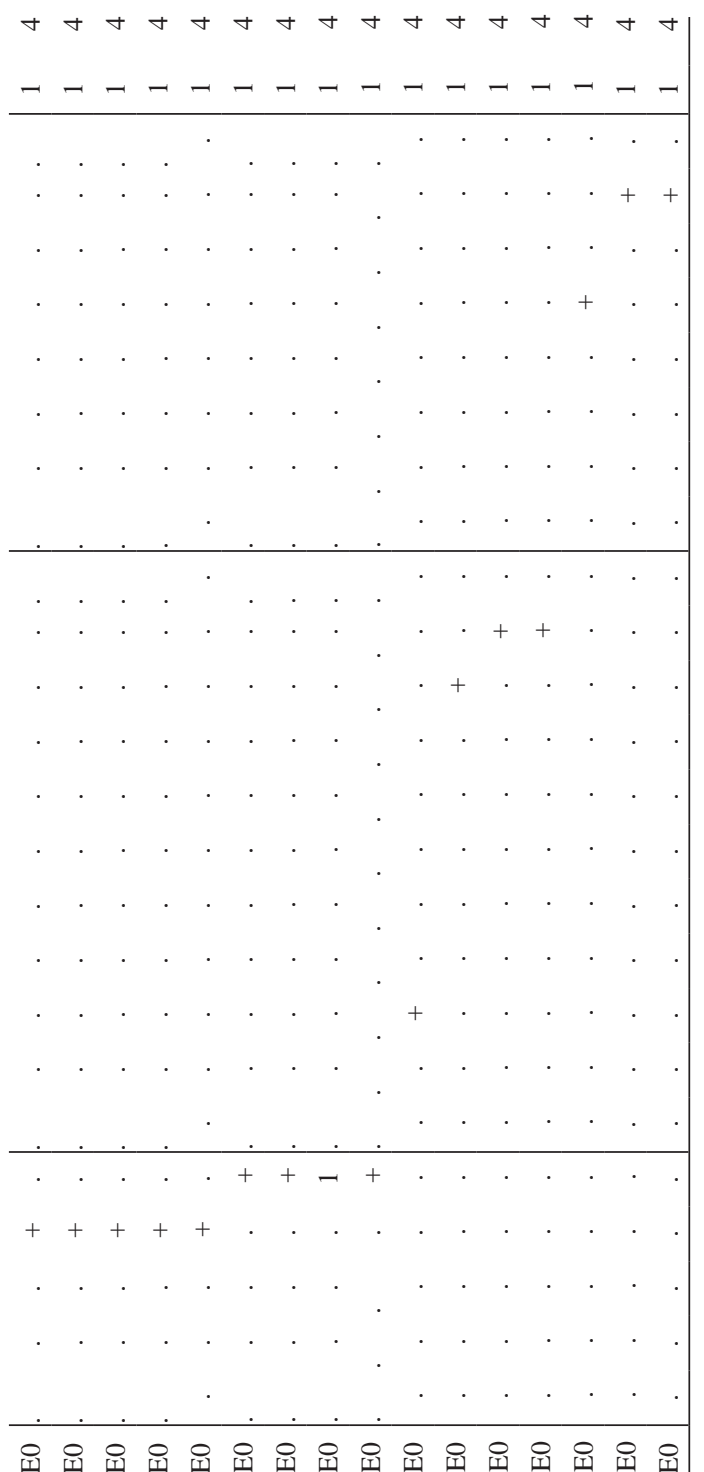

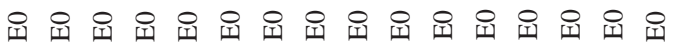

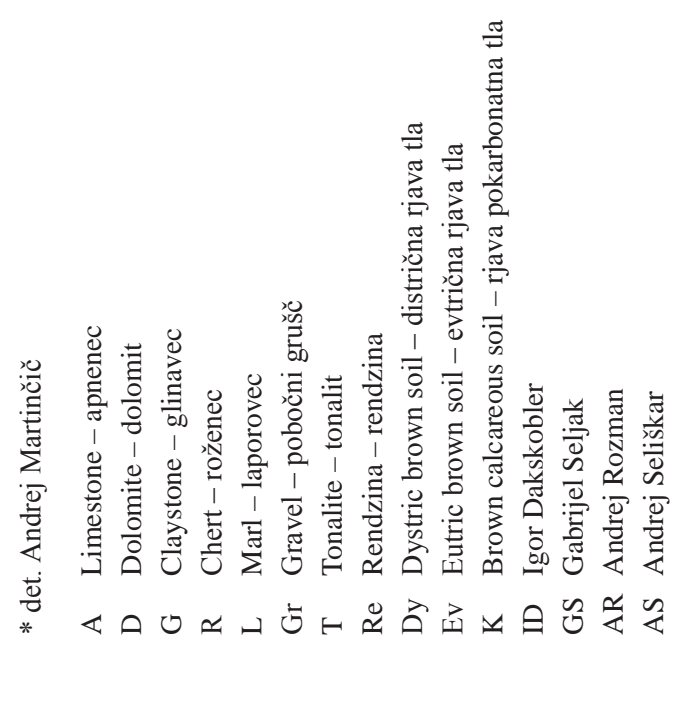


Table 3 / Tabela 3: Huperzio selagi-Alnetum viridis ass. nov.

\begin{tabular}{|c|c|c|c|c|c|c|c|c|c|c|}
\hline & Number of relevé (Zaporedna štev. popisa) & & 1 & 2 & 3 & 4 & 5 & 6 & & \\
\hline & $\begin{array}{l}\text { Database number of relevé } \\
\text { (Delovna številka popisa) }\end{array}$ & & 244808 & 244812 & 244809 & 244811 & 244810 & 244813 & & \\
\hline & Elevation in m (Nadmorska višina v m) & & 1640 & 1650 & 1645 & 1640 & 1650 & 1670 & & \\
\hline & Aspect (Lega) & & $\mathrm{N}$ & $\mathrm{NE}$ & NW & $\mathrm{N}$ & $\mathrm{NE}$ & $\mathrm{NE}$ & & \\
\hline & Slope in degrees (Nagib v stopinjah) & & 35 & 40 & 35 & 45 & 35 & 50 & & \\
\hline & Parent material (Matična podlaga) & & AN & $\mathrm{AN}$ & AN & $\mathrm{AN}$ & $\mathrm{AN}$ & $\mathrm{AN}$ & & \\
\hline & Soil (Tla) & & Dy & $\mathrm{Ra}$ & Dy & $\mathrm{Ra}$ & Dy & $\mathrm{Ra}$ & & \\
\hline & Stoniness in \% (Kamnitost v \%) & & 5 & 5 & 5 & 40 & 5 & 40 & & \\
\hline & $\begin{array}{l}\text { Cover of shrub layer in \% } \\
\text { (Zastiranje grmovne plasti v \%): }\end{array}$ & E2 & 80 & 90 & 80 & 80 & 80 & 70 & & \\
\hline & $\begin{array}{l}\text { Cover of herb layer in \% } \\
\text { (Zastiranje zeliščne plasti v \%): }\end{array}$ & E1 & 80 & 90 & 80 & 80 & 80 & 70 & & \\
\hline & $\begin{array}{l}\text { Cover of moss layer in \% } \\
\text { (Zastiranje mahovne plasti v \%) }\end{array}$ & E0 & 30 & 20 & 30 & 10 & 10 & 10 & & \\
\hline & Number of species (Število vrst) & & 42 & 33 & 32 & 51 & 32 & 36 & & \\
\hline & Relevé area (Velikost popisne ploskve) & $\mathrm{m}^{2}$ & 100 & 100 & 100 & 100 & 100 & 100 & & \\
\hline & $\begin{array}{l}\text { Date of taking relevé } \\
\text { (Datum popisa) }\end{array}$ & & $\begin{array}{l}9 / 11 / \\
2012\end{array}$ & $\begin{array}{l}9 / 11 / \\
2012\end{array}$ & $\begin{array}{l}9 / 11 / \\
2012\end{array}$ & $\begin{array}{l}9 / 11 / \\
2012\end{array}$ & $\begin{array}{l}9 / 11 / \\
2012\end{array}$ & $\begin{array}{l}9 / 11 / \\
2012\end{array}$ & & \\
\hline & Locality (Nahajališče) & & Komen & Komen & Komen & Komen & Komen & Komen & & \\
\hline & Quadrant (Kvadrant) & & $9555 / 3$ & $9555 / 3$ & $9555 / 3$ & $9555 / 3$ & $9555 / 3$ & $9555 / 3$ & & \\
\hline & Coordinate GK Y (D-48) & $\mathrm{m}$ & 488470 & 488621 & 488904 & 488728 & 488831 & 488544 & & \\
\hline & Coordinate GK X (D-48) & $\mathrm{m}$ & 141639 & 141563 & 141655 & 141557 & 141478 & 141608 & & \\
\hline & Author of the relevé (Avtor popisa) & & ID, AS & ID, AS & ID, AS & ID, AS & ID, AS & ID, AS & Pr. & Fr. \\
\hline & \multicolumn{10}{|c|}{ Diagnostic species of the association (Diagnostične vrste asociacije) } \\
\hline VP & Vaccinium vitis-idaea & E1 & + & + & + & + & + & 2 & & 100 \\
\hline ML & *Sphagnum russowii & E0 & 2 & 1 & 2 & + & . & + & 5 & \\
\hline VP & Lycopodium annotinum & E1 & 1 & 1 & 1 & 1 & . & + & 5 & 83 \\
\hline VP & Huperzia selago & E1 & + & + & . & + & + & 1 & 5 & 83 \\
\hline VP & Rhytidiadelphus loreus & E0 & 1 & + & 1 & 1 & . & . & 4 & 66 \\
\hline JT & Festuca varia & E1 & + & + & . & . & + & + & 4 & 67 \\
\hline QF & Festuca guestfalica & E1 & + & . & . & 1 & + & 2 & 4 & 67 \\
\hline VP & Thelypteris limbosperma & E1 & + & 1 & 1 & . & . & . & 3 & 50 \\
\hline $\mathrm{AV}$ & Primula villosa & E1 & $\mathrm{r}$ & . & . & . & $\mathrm{r}$ & . & 2 & 33 \\
\hline JT & Primula minima & E1 & $\mathrm{r}$ & . & . & . & . & 3 & 2 & 33 \\
\hline BA & Betulo carpaticae-Alnetea viridis & & & & & & & & & \\
\hline & Alnus viridis & $\mathrm{E} 2 \mathrm{~b}$ & 4 & 5 & 5 & 5 & 5 & 4 & 6 & 100 \\
\hline & Alnus viridis & E2a & + & . & . & . & . & 1 & 2 & 33 \\
\hline & Alnus viridis & E1 & . & . & . & . & . & + & 1 & 17 \\
\hline \multicolumn{11}{|c|}{ MuA Mulgedio-Aconitetea } \\
\hline & Athyrium filix-femina & E1 & + & + & . & + & + & . & 4 & 67 \\
\hline & Veratrum album subsp. album & E1 & + & & + & $\mathrm{r}$ & & & 3 & 50 \\
\hline & Viola biflora & E1 & + & . & . & 2 & . & + & 3 & 50 \\
\hline & Stellaria nemorum & E1 & . & . & + & 1 & . & . & 2 & 33 \\
\hline & Polygonatum verticillatum & E1 & . & + & . & . & . & . & 1 & 17 \\
\hline & Saxifraga rotundifolia & E1 & . & . & . & + & . & . & 1 & 17 \\
\hline & Adenostyles alliariae & E1 & . & . & . & r & . & . & 1 & 17 \\
\hline \multirow[t]{4}{*}{ SSC } & Sambuco-Salicion capreae & & & & & & & & & \\
\hline & Sorbus aucuparia & E3a & . & . & . & . & + & . & 1 & 17 \\
\hline & Sorbus aucuparia & $\mathrm{E} 2 \mathrm{~b}$ & + & 1 & + & + & . & + & 5 & 83 \\
\hline & Sorbus aucuparia & E2a & . & + & . & + & + & . & 3 & 50 \\
\hline
\end{tabular}




\begin{tabular}{|c|c|c|c|c|c|c|c|c|c|c|}
\hline & Number of relevé (Zaporedna štev. popisa) & & 1 & 2 & 3 & 4 & 5 & 6 & Pr. & Fr. \\
\hline & Sorbus aucuparia & E1 & + & . & . & + & . & + & 3 & 50 \\
\hline & Rubus idaeus & $\mathrm{E} 2 \mathrm{a}$ & . & + & . & + & 1 & . & 3 & 50 \\
\hline \multirow[t]{23}{*}{ VP } & Vacinio-Piceetea & & & & & & & & & \\
\hline & Vaccinium myrtillus & E1 & 4 & 3 & 2 & 1 & 3 & 3 & 6 & 100 \\
\hline & Calamagrostis villosa & E1 & 4 & 4 & 4 & + & 3 & + & 6 & 100 \\
\hline & Avenella flexuosa & E1 & + & 1 & 2 & + & + & + & 6 & 100 \\
\hline & Phegopteris connectilis & E1 & 2 & 2 & 2 & 1 & 1 & . & 5 & 83 \\
\hline & Picea abies & E3a & + & . & . & . & + & . & 2 & 33 \\
\hline & Picea abies & E2 & 1 & + & + & + & + & 1 & 6 & 100 \\
\hline & Picea abies & E1 & + & + & + & . & . & . & 3 & 50 \\
\hline & Dryopteris dilatata & E1 & 2 & 3 & 3 & 3 & 1 & . & 5 & 83 \\
\hline & Oxalis acetosella & E1 & 1 & 1 & 2 & 2 & + & . & 5 & 83 \\
\hline & Luzula sylvatica & E1 & 1 & + & 4 & + & . & . & 4 & 67 \\
\hline & Homogyne alpina & E1 & + & + & 1 & + & . & . & 4 & 67 \\
\hline & Solidago virgaurea & E1 & . & + & . & + & . & + & 3 & 50 \\
\hline & Luzula luzuloides subsp. luzuloides & E1 & . & . & + & + & 1 & . & 3 & 50 \\
\hline & Dryopteris expansa & E1 & + & + & & & & & 2 & 33 \\
\hline & Calamagrostis arundinacea & E1 & + & . & . & . & 1 & . & 2 & 33 \\
\hline & Gentiana asclepiadea & E1 & . & 1 & 1 & . & . & . & 2 & 33 \\
\hline & Valeriana tripteris & E1 & . & . & . & + & . & + & 2 & 33 \\
\hline & Clematis alpina & $\mathrm{E} 2 \mathrm{a}$ & . & . & . & . & r & + & 2 & 33 \\
\hline & Blechnum spicant & E1 & + & . & . & . & . & . & 1 & 17 \\
\hline & Abies alba & E1 & $\mathrm{r}$ & . & . & . & . & . & 1 & 17 \\
\hline & Larix decidua & $\mathrm{E} 2 \mathrm{a}$ & . & . & . & . & . & $\mathrm{r}$ & 1 & 17 \\
\hline & Larix decidua & E1 & . & . & . & . & . & $\mathrm{r}$ & 1 & 17 \\
\hline \multirow[t]{4}{*}{ EP } & Erico-Pinetea & & & & & & & & & \\
\hline & Rhododendron hirsutum & E2a & + & . & . & + & $r$ & 1 & 4 & 67 \\
\hline & Pinus mugo & E2 & . & + & . & . & + & + & 3 & 50 \\
\hline & Erica carnea & E1 & . & . & . & . & + & + & 2 & 33 \\
\hline \multirow[t]{3}{*}{ FS } & Fagetalia sylvaticae & & & & & & & & & \\
\hline & Dryopteris affinis & E1 & . & . & . & + & + & . & 2 & 33 \\
\hline & Lilium martagon & E1 & . & . & . & . & . & + & 1 & 17 \\
\hline \multirow[t]{4}{*}{ QF } & Querco-Fagetea & & & & & & & & & \\
\hline & Anemone nemorosa & E1 & 1 & + & + & + & & + & 5 & 83 \\
\hline & Melampyrum pratense & E1 & $\mathrm{r}$ & . & . & . & + & . & 2 & 33 \\
\hline & Carex brizoides & E1 & . & . & . & . & + & . & 1 & 17 \\
\hline \multirow[t]{3}{*}{ ES } & Elyno-Seslerietea & & & & & & & & & \\
\hline & Gentianella anisodonta & E1 & . & . & . & . & . & + & 1 & 17 \\
\hline & Aster bellidiastrum & E1 & . & . & . & . & . & $\mathrm{r}$ & 1 & 17 \\
\hline \multirow[t]{3}{*}{ LV } & Loiseleurio-Vaccinietea & & & & & & & & & \\
\hline & Vaccinium gaultherioides & E1 & . & . & . & . & . & 3 & 1 & 17 \\
\hline & Empetrum hermaphroditum & E1 & . & . & . & . & . & + & 1 & 17 \\
\hline \multirow[t]{5}{*}{$\mathrm{CU}$} & Calluno-Ulicetea & & & & & & & & & \\
\hline & Campanula barbata & E1 & r & . & . & $\mathrm{r}$ & . & $\mathrm{r}$ & 3 & 50 \\
\hline & Calluna vulgaris & E1 & . & . & . & . & + & + & 2 & 33 \\
\hline & Carex pilulifera & E1 & . & . & . & . & . & + & 1 & 17 \\
\hline & Festuca filiformis & E1 & . & . & . & . & . & + & 1 & 17 \\
\hline \multirow[t]{2}{*}{ EA } & Epilobietea angustifolii & & & & & & & & & \\
\hline & Chamaenerion angustifolium & E1 & . & . & . & . & + & . & 1 & 17 \\
\hline \multirow[t]{4}{*}{ AT } & Asplenietea trichomanis & & & & & & & & & \\
\hline & Cystopteris fragilis & E1 & . & . & . & + & . & . & 1 & 17 \\
\hline & Paederota lutea & E1 & . & . & . & + & . & . & 1 & 17 \\
\hline & Hieracium schmidtii & E1 & . & . & . & . & . & $\mathrm{r}$ & 1 & 17 \\
\hline
\end{tabular}


Number of relevé (Zaporedna štev. popisa)

1
2 3 4 5 6 Pr. Fr.

JT Juncetea trifidi

Agrostis rupestris

Pulsatilla alpina subsp. alba

E1

E1

Campanula alpina

MA Molinio-Arrhenatheretea

Deschampsia cespitosa

MC Montio-Cardaminetea

Saxifraga stellaris subsp. prolifera

Cardamine amara

TR Thlaspietea rotundifolii

Campanula cochleariifolia

Hieracium bifidum

Cardamine resedifolia

ML Moses and lichens (Mahovi in lišaji)

Polytrichum formosum

Dicranum scoparium

Rhytidiadelphus triquetrus

*Diplophyllum albicans

*Pogonatum unigerum

Tortella tortuosa

*Sphagnum subnitens

Cetraria islandica

Hylocomium splendens

*Grimmia sp.

*Cephalozia lacinulata

*Calypogeia azurea

*Nardia scalaris

*Amphidium mougeotii

Plagiothecium undulatum

*Hypnum cupressiforme var. cupressiforme

*Calypogeia integristipula

*Tortella densa

*Brachythecium plumosum

*Polytrichum juniperinum

* Racomitrium lanuginosum

*Polytrichum alpinum

*Rhizomnium punctatum

*Brachytehecium rutabulum

Rhizocarpon geographicum
E1

E1

E1

E1

E1

E1

E1

E0

E0

E0

E0

E0

E0

E0

E0

E0

E0

E0

E0

E0

E0

E0

E0

E0

E0

E0

E0.

E0

E0

E0

E0

E0
233

$1 \quad 17$

$1 \quad 17$

350

233

117

$2 \quad 33$

$1 \quad 17$

117

583

583

$4 \quad 67$

350

350

350

233

233

233

117

$1 \quad 17$

$1 \quad 17$

$1 \quad 17$

$1 \quad 17$

117

117

$1 \quad 17$

117

117

117

117

117

117

117

$1 \quad 17$

* det. Andrej Martinčič

AN Andesite - andezit

Ra Ranker - ranker

Dy Dystric brown soil - distrična rjava tla

ID Igor Dakskobler

AS Andrej Seliškar 
Table 4: Synoptic table of the macroassociation Alnetum viridis s. lat.

Tabela 4: Sintezna tabela makroasociacije Alnetum viridis s. lat.

\begin{tabular}{|c|c|c|c|c|c|c|c|c|c|}
\hline Successive number (Zaporedna številka) & 1 & 2 & 3 & 4 & 5 & 6 & 7 & 8 & 9 \\
\hline Number of relevés (Število popisov) & 4 & 18 & 54 & 24 & 107 & 23 & 24 & 53 & 6 \\
\hline Sign for the syntaxa (Oznaka sintaksonov) & $\begin{array}{l}\frac{y}{1} \\
\frac{Z}{2}\end{array}$ & 点 & $\begin{array}{l}\mathbb{1} \\
\stackrel{\pi}{\pi} \\
\stackrel{2}{<}\end{array}$ & $\sum_{i}^{\infty}$ & 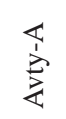 & 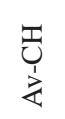 & $\underset{4}{4}$ & 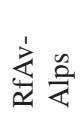 & 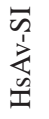 \\
\hline Author (Avtor) & Aich & ID & Kar & ID & Kar & $\mathrm{BB}$ & Kar & Po & ID \\
\hline
\end{tabular}

Diagnostic species of the association Rhododendro hirsuti-Alnetum viridis

Diagnostične vrste asociacije Rhododendro hirsuti-Alnetum viridis

\begin{tabular}{lll|cccc|} 
MuA Primula elatior & E1 & 75 & 50 & 35 & 4 \\
VP Homogyne sylvestris & E1 & 50 & 61 &. &. \\
TA Acer pseudoplatanus & E2 & 50 & 33 & 37 & 38 \\
TA Acer pseudoplatanus & E1 &. & 17 &. & 29 \\
MuA Aconitum lycoctonum s. lat. & E1 & 25 & 100 & 43 & 21 \\
VP Polystichum lonchitis & E1 & 25 & 83 & 50 & 13 \\
EP Rhododendron hirsutum & E2a & 25 & 56 & 39 & 17 \\
VP Valeriana tripteris & E1 &. & 83 & 37 & 13 \\
AT Asplenium viride & E1 & $\cdot$ & 78 & 32 & 8 \\
FS Galeobdolon flavidum (G. luteum agg.) & E1 & $\cdot$ & 67 & 44 & 25 \\
MuA Thalictrum aquilegiifolium & E1 & $\cdot$ & 67 & 33 & 4 \\
BA Salix waldsteiniana & E2 & $\cdot$ & 56 & 13 & 21 \\
BA Sorbus chamaemespilus & E2 & $\cdot$ & 56 & 13 & 8 \\
TR Adenostyles glabra & E1 & $\cdot$ & 56 & 11 & 25 \\
TR Cystopteris montana & E1 &. & 50 &. &. \\
\hline
\end{tabular}

Diagnostic species of the association Rhododendro ferruginei-Alnetum viridis

Diagnostične vrste asociacije Rhododendro ferruginei-Alnetum viridis

\begin{tabular}{|c|c|c|c|c|c|c|c|c|c|c|c|}
\hline VP & Dryopteris dilatata & E1 & 100 & 61 & 44 & 88 & 40 & 65 & 54 & 81 & 83 \\
\hline VP & Vaccinium myrtillus & E1 & 75 & 61 & 59 & 38 & 40 & 39 & 92 & 72 & 100 \\
\hline SSC & Rubus idaeus & E2a & 50 & 22 & 46 & 96 & 48 & 26 & 21 & 64 & 50 \\
\hline VP & Rhododendron ferrugineum & E1 & . & . & 2 & . & 22 & 22 & 92 & 79 & . \\
\hline JT & Potentilla aurea & E1 & . & . & 13 & . & 15 & 17 & 67 & . & . \\
\hline \multirow[t]{3}{*}{ JT } & Anthoxanthum odoratum agg. & E1 & . & 6 & 11 & . & 12 & . & 54 & 17 & . \\
\hline & \multicolumn{11}{|c|}{ Diagnostic species of the association Huperzio-Alnetum viridis } \\
\hline & \multicolumn{11}{|c|}{ Diagnostične vrste asociacije Huperzio-Alnetum viridis } \\
\hline VP & Vaccinium vitis-idaea & E1 & . & 33 & 11 & . & 7 & 17 & 42 & 9 & 100 \\
\hline VP & Huperzia selago & E1 & . & 11 & . & 4 & . & 4 & . & 2 & 83 \\
\hline VP & Lycopodium annotinum & E1 & . & 33 & 13 & . & . & . & 13 & . & 83 \\
\hline VP & Sphagnum russowii & E0 & . & . & . & . & . & . & . & . & 83 \\
\hline VP & Rhytidiadelphus loreus & E0 & . & 11 & . & . & . & . & . & . & 67 \\
\hline VP & Thelypteris limbosperma & E1 & . & . & 11 & 25 & 18 & . & 8 & 8 & 50 \\
\hline JT & Festuca varia & E1 & . & . & . & . & . & . & . & 2 & 67 \\
\hline QF & Festuca guestfalica & E1 & . & . & . & . & . & . & . & . & 67 \\
\hline JT & Primula minima & E1 & . & . & . & . & . & . & . & . & 33 \\
\hline $\mathrm{AV}$ & Primula villosa & E1 & . & . & . & . & . & . & . & . & 33 \\
\hline \multirow[t]{6}{*}{ BA } & Betulo carpaticae-Alnetea vir & & & & & & & & & & \\
\hline & Alnus viridis & $\mathrm{E} 2 \mathrm{~b}$ & 100 & 100 & 100 & 100 & 100 & 100 & 100 & 100 & 100 \\
\hline & Ribes alpinum & E2a & 50 & . & . & 8 & . & . & . & . & . \\
\hline & Salix appendiculata & $\mathrm{E} 2 \mathrm{~b}$ & 25 & 50 & 28 & 29 & 18 & 22 & 4 & 8 & . \\
\hline & Salix hastata & E2 & 25 & . & 6 & . & 7 & . & . & . & . \\
\hline & Ribes petraeum & E1 & . & . & 2 & . & 1 & 22 & 4 & . & . \\
\hline \multicolumn{12}{|c|}{ MuA Mulgedio-Aconitetea } \\
\hline & Viola biflora & E1 & 100 & 89 & 89 & 29 & 66 & 87 & 50 & 28 & 50 \\
\hline & Saxifraga rotundifolia & E1 & 100 & 72 & 78 & 38 & 56 & 65 & 25 & 15 & 17 \\
\hline
\end{tabular}


Successive number (Zaporedna številka)

Chaerophyllum hirsutum

Adenostyles alliariae

Stellaria nemorum s.str.

Cicerbita alpina

Silene dioica

Aconitum degenii subsp. paniculatum

Senecio nemorensis

Lamium maculatum

Polygonatum verticillatum

Doronicum austriacum

Streptopus amplexifolius

Cirsium carniolicum

Rumex arifolius

Geranium sylvaticum

Veratrum album s. lat.

Ranunculus platanifolius

Hypericum maculatum

Senecio ovatus

Carduus personata

Lathyrus occidentalis var. montanus

Pleurospermum austriacum

Allium victorialis

Milium effusum

Athyrium distentifolium

Agropyron caninum

Geum rivale

Thalictrum aquilegiifolium

Senecio cacaliaster

Athyrium filix-femina

Crepis paludosa

Heracleum sphondylium subsp. montanum (inc. H. pollinianum)

Salix glabra

Tephroseris crispa

Poa hybrida (inc. P.chaixii)

Aconitum tauricum (inc. A. napellus agg.)

Cirsium waldsteinii

Myrrhis odorata

Chaerophyllum villarsii

Epilobium alpestre

Phyteuma ovatum

Alchemilla xanthochlora

Alchemilla venosula (A. gracillima)

Carduus carduelis

Crepis pyrenaica

Silene vulgaris subsp. antelopum

Pedicularis recutita

Circaea alpina

Knautia dipsacifolia

Peucedanum ostruthium

Centaurea montana

Schrophularia scopolii

Alchemilla monticola

Rumex alpinus

$\begin{array}{lccccccccc} & 1 & 2 & 3 & 4 & 5 & 6 & 7 & 8 & 9 \\ \text { E1 } & 100 & 67 & 70 & 29 & 36 & 52 & 13 & 8 & . \\ \text { E1 } & 100 & 67 & 70 & 50 & 56 & 96 & 33 & 25 & 17 \\ \text { E1 } & 100 & 39 & 48 & 63 & 57 & 74 & 21 & 34 & 33 \\ \text { E1 } & 100 & 22 & 43 & 17 & 20 & 74 & . & 4 & . \\ \text { E1 } & 100 & 22 & 26 & 8 & 8 & 26 & . & . & . \\ \text { E1 } & 100 & 17 & 15 & . & . & 35 & . & . & . \\ \text { E1 } & 100 & 17 & . & 4 & . & 35 & . & 40 & . \\ \text { E1 } & 100 & . & . & 13 & . & . & . & . & . \\ \text { E1 } & 75 & 61 & 33 & 75 & 7 & 9 & 17 & 8 & 17 \\ \text { E1 } & 75 & 50 & 17 & 13 & 14 & . & 8 & 15 & . \\ \text { E1 } & 75 & 6 & . & 13 & . & 13 & . & 2 & . \\ \text { E1 } & 75 & 6 & . & . & . & . & . & . & . \\ \text { E1 } & 50 & 28 & 28 & 42 & 56 & 65 & 46 & 30 & . \\ \text { E1 } & 25 & 67 & 65 & 8 & 50 & 61 & 4 & 6 & . \\ \text { E1 } & 25 & 61 & 61 & 83 & 62 & 65 & 71 & 36 & 50 \\ \text { E1 } & 25 & 39 & 13 & 17 & 13 & 39 & 8 & 8 & . \\ \text { E1 } & 25 & 39 & 59 & 38 & 32 & 13 & 29 & 4 & . \\ \text { E1 } & 25 & 33 & 67 & 58 & 51 & . & 63 & . & . \\ \text { E1 } & 25 & 22 & 13 & 25 & 3 & 9 & 4 & . & . \\ \text { E1 } & 25 & 6 & . & . & . & . & . & . & . \\ \text { E1 } & 25 & 6 & . & 4 & . & . & . & . & . \\ \text { E1 } & 25 & 6 & 2 & . & 1 & . & . & 2 & . \\ \text { E1 } & 25 & 6 & 6 & 4 & 5 & 22 & . & 4 & . \\ \text { E1 } & 25 & . & 28 & 13 & 67 & 26 & 42 & 45 & . \\ \text { E1 } & 25 & . & . & . & . & 4 & . & 2 & . \\ \text { E1 } & . & 67 & 43 & 50 & 6 & 17 & 4 & 8 & . \\ \text { E1 } & . & 67 & 33 & 4 & 3 & 4 & . & 2 & . \\ \text { E1 } & . & 61 & 4 & 46 & . & . & . & . & . \\ \text { E1 } & . & 50 & 48 & 96 & 12 & 9 & 29 & 43 & 67 \\ \text { E1 } & . & 50 & 22 & 25 & 6 & 13 & 4 & . & . \\ & & & & & & & & & . \\ \end{array}$

\begin{tabular}{|c|c|c|c|c|c|c|c|c|}
\hline E1 & . & 33 & 17 & 8 & 5 & 4 & . & 6 \\
\hline E2a & . & 33 & 6 & 4 & . & . & . & . \\
\hline E1 & . & 22 & 4 & . & . & . & . & . \\
\hline E1 & . & 22 & 19 & 8 & 1 & 13 & . & 2 \\
\hline E1 & . & 22 & 32 & 4 & 30 & 52 & 38 & 4 \\
\hline E1 & . & 22 & . & . & . & . & . & . \\
\hline E1 & . & 17 & . & . & . & . & . & . \\
\hline E1 & . & 17 & 11 & 21 & 14 & . & 8 & . \\
\hline E1 & . & 17 & 22 & 4 & 10 & 35 & 4 & . \\
\hline E1 & . & 11 & . & 4 & . & 26 & . & 2 \\
\hline E1 & . & 11 & . & 25 & . & . & . & . \\
\hline E1 & . & 11 & . & . & . & . & . & . \\
\hline E1 & . & 11 & . & . & . & . & . & . \\
\hline E1 & . & 6 & 4 & . & 1 & . & . & . \\
\hline E1 & . & 6 & 15 & . & 19 & 4 & . & 17 \\
\hline E1 & . & 6 & . & . & . & 13 & 38 & . \\
\hline E1 & . & 6 & . & . & . & . & . & . \\
\hline E1 & . & . & 33 & . & 7 & 9 & 4 & . \\
\hline E1 & . & . & 24 & . & 63 & 87 & 58 & 28 \\
\hline E1 & . & . & 6 & . & . & . & . & . \\
\hline E1 & . & . & . & 8 & . & . & . & . \\
\hline E1 & . & . & . & 4 & . & . & . & . \\
\hline E1 & . & . & . & 4 & . & . & . & 6 \\
\hline
\end{tabular}




\begin{tabular}{|c|c|c|c|c|c|c|c|c|c|c|c|}
\hline & Successive number (Zaporedna številka) & & 1 & 2 & 3 & 4 & 5 & 6 & 7 & 8 & 9 \\
\hline & Anthriscus nitidus & E1 & . & . & . & 4 & . & . & . & . & . \\
\hline & Cirsium helenioides & E1 & . & . & . & . & 3 & 4 & 4 & . & . \\
\hline & Achillea macrophylla & E1 & . & . & . & . & . & 70 & . & 4 & . \\
\hline & Cerinthe glabra & E1 & . & . & . & . & . & 4 & . & . & . \\
\hline & Chaerophyllum aureum & E1 & . & . & . & . & . & 4 & . & . & . \\
\hline & Senecio alpinus & E1 & . & . & . & . & . & 4 & . & . & . \\
\hline & Cirsium spinosissimum & E1 & . & . & . & . & . & . & . & 8 & . \\
\hline & Salix helvetica & E1 & . & . & . & . & . & . & . & 6 & . \\
\hline \multirow[t]{10}{*}{ SSC } & Sambuco-Salicion capreae & & & & & & & & & & \\
\hline & Sorbus aucuparia & $\mathrm{E} 2 \mathrm{~b}$ & 75 & 39 & 56 & 50 & 28 & 39 & 33 & 34 & 100 \\
\hline & Sorbus aucuparia & E2a & . & 6 & . & 8 & . & . & . & . & 50 \\
\hline & Sorbus aucuparia & E1 & . & 22 & . & 13 & . & . & . & . & 50 \\
\hline & Urtica dioica & E1 & 25 & 17 & 15 & 38 & 20 & 17 & . & 6 & . \\
\hline & Fragaria vesca & E1 & . & 6 & 19 & . & 13 & 4 & 4 & 4 & . \\
\hline & Galeopsis speciosa & E1 & . & 6 & . & 21 & . & . & . & 13 & . \\
\hline & Sambucus racemosa & E1 & . & . & 7 & . & 1 & . & . & . & . \\
\hline & Salix caprea & E3a & . & . & . & 4 & . & . & . & . & . \\
\hline & Salix caprea & $\mathrm{E} 2 \mathrm{~b}$ & . & . & . & 4 & . & . & . & . & . \\
\hline RP & Viburnum opulus & E2a & . & . & . & 4 & . & . & . & . & . \\
\hline $\mathrm{RP}$ & Rosa canina & $\mathrm{E} 2 \mathrm{a}$ & . & . & . & 4 & . & . & . & . & . \\
\hline \multirow[t]{35}{*}{ VP } & Vaccinio-Piceetea & & & & & & & & & & \\
\hline & Calamagrostis villosa & E1 & 100 & 50 & 19 & 13 & 43 & 52 & 75 & 38 & 100 \\
\hline & Luzula sylvatica & E1 & 75 & 61 & 46 & 33 & 22 & 22 & 4 & 21 & 67 \\
\hline & Aposeris foetida & E1 & 50 & 44 & 37 & 25 & 13 & . & . & . & . \\
\hline & Homogyne alpina & E1 & 50 & 28 & 41 & 4 & 33 & 43 & 79 & 38 & 67 \\
\hline & Lonicera nigra & $\mathrm{E} 2 \mathrm{a}$ & 50 & 17 & 13 & . & 1 & 13 & . & . & . \\
\hline & Solidago virgaurea s. lat. & E1 & 25 & 50 & 43 & 4 & 59 & 52 & 63 & 28 & 50 \\
\hline & Phegopteris connectilis & E1 & 25 & 50 & 15 & 58 & 10 & 4 & 13 & 47 & 83 \\
\hline & Picea abies & E3 & . & . & . & 17 & . & . & . & . & . \\
\hline & Picea abies & E2b & 25 & 56 & 35 & 29 & 26 & 9 & 46 & 11 & 100 \\
\hline & Picea abies & E1 & . & 22 & . & 4 & . & . & . & . & 50 \\
\hline & Gentiana asclepiadea & E1 & 25 & 44 & 24 & 79 & 4 & . & 4 & . & 33 \\
\hline & Clematis alpina & E2a & 25 & 44 & 11 & . & 1 & 4 & . & 2 & 33 \\
\hline & Luzula luzuloides subsp. luzuloides & E1 & 25 & 22 & 6 & 50 & 25 & . & 13 & 21 & 50 \\
\hline & Veronica urticifolia & E1 & 25 & 17 & 17 & 13 & 4 & 9 & . & 4 & . \\
\hline & Larix decidua & E3 & . & 6 & . & . & . & . & . & . & . \\
\hline & Larix decidua & E2 & . & 50 & 30 & . & 9 & . & 25 & 11 & 17 \\
\hline & Oxalis acetosella & E1 & . & 56 & 54 & 63 & 42 & 56 & 33 & 43 & 83 \\
\hline & Rosa pendulina & $\mathrm{E} 2 \mathrm{a}$ & . & 44 & 11 & 17 & 3 & 9 & . & 2 & . \\
\hline & Gymnocarpium dryopteris & E1 & . & 44 & 11 & 8 & 13 & . & 4 & 11 & . \\
\hline & Saxifraga cuneifolia & E1 & . & 33 & . & 8 & . & . & . & 2 & . \\
\hline & Dryopteris expansa & E1 & . & 22 & . & 21 & . & . & . & . & 33 \\
\hline & Maianthemum bifolium & E1 & . & 17 & 4 & 33 & 2 & . & 8 & 13 & . \\
\hline & Calamagrostis arundinacea & E1 & . & 11 & . & 42 & . & . & . & 2 & 33 \\
\hline & Lonicera caerulea & $\mathrm{E} 2 \mathrm{a}$ & . & 11 & 11 & . & 4 & 39 & 13 & 9 & . \\
\hline & Abies alba & E2 & . & 11 & . & . & . & . & . & 2 & . \\
\hline & Abies alba & E1 & . & 11 & . & . & . & . & . & . & 17 \\
\hline & Pyrola rotundifolia & E1 & . & 11 & . & . & . & . & . & . & $\cdot$ \\
\hline & Luzula luzuloides subsp. rubella & E1 & . & 6 & . & 17 & . & . & . & . & . \\
\hline & Luzula luzulina & E1 & . & 6 & . & 4 & . & . & . & . & . \\
\hline & Hieracium murorum & E1 & . & . & 17 & . & 13 & 4 & 21 & 9 & . \\
\hline & Avenella flexuosa & E1 & . & . & 9 & 4 & 32 & . & 71 & 42 & 100 \\
\hline & Pinus cembra & E2 & . & . & 6 & . & 3 & . & 29 & 6 & . \\
\hline & Luzula pilosa & E1 & . & . & . & 13 & . & . & . & 2 & . \\
\hline & Circaea alpina & E1 & . & . & . & 8 & . & . & . & . & . \\
\hline
\end{tabular}




\begin{tabular}{|c|c|c|c|c|c|c|c|c|c|c|c|}
\hline & Successive number (Zaporedna številka) & & 1 & 2 & 3 & 4 & 5 & 6 & 7 & 8 & 9 \\
\hline & Blechnum spicant & E1 & . & . & . & 8 & . & . & . & 2 & 17 \\
\hline & Melampyrum sylvaticum & E1 & . & . & . & 4 & . & . & . & . & . \\
\hline & Linnaea borealis & E1 & . & . & . & . & . & 4 & . & . & . \\
\hline & Festuca flavescens & E1 & . & . & . & . & . & . & . & 15 & . \\
\hline \multirow[t]{14}{*}{ EP } & Erico-Pinetea & & & & & & & & & & \\
\hline & Aquilegia nigricans & E1 & 25 & 11 & . & . & . & . & . & . & . \\
\hline & Pinus mugo & E2a & 25 & 11 & 17 & . & 9 & . & . & 4 & 50 \\
\hline & Rubus saxatilis & E1 & . & 78 & 20 & 8 & 2 & 4 & . & 2 & . \\
\hline & Calamagrostis varia & E1 & . & 28 & 13 & . & 1 & . & . & 4 & . \\
\hline & Cirsium erisithales & E1 & . & 28 & . & 8 & . & . & . & 2 & . \\
\hline & Carex ornithopoda & E1 & . & 17 & . & . & . & . & . & . & . \\
\hline & Erica carnea & E1 & . & 11 & . & . & . & . & . & . & 33 \\
\hline & Rhodothamnus chamaecistus & E1 & . & 6 & . & . & . & . & . & . & . \\
\hline & Galium austriacum & E1 & . & 6 & . & . & . & . & . & . & . \\
\hline & Juniperus alpina & E1 & . & . & 6 & . & 3 & . & 25 & 8 & . \\
\hline & Aquilegia attrata & E1 & . & . & 6 & . & . & . & . & . & . \\
\hline & Molinia caerulea agg. & E1 & . & . & . & . & 4 & . & 4 & 2 & . \\
\hline & Aquilegia alpina & E1 & . & . & . & . & . & 4 & . & . & . \\
\hline \multirow[t]{10}{*}{$\mathrm{AF}$} & Aremonio-Fagion & & & & & & & & & & \\
\hline & Lamium orvala & E1 & 50 & . & . & . & . & . & . & . & . \\
\hline & Cardamine enneaphyllos & E1 & 25 & 44 & 26 & 17 & . & . & . & . & . \\
\hline & Cyclamen purpurascens & E1 & 25 & 28 & . & 4 & . & . & . & . & . \\
\hline & Helleborus niger & E1 & 25 & 22 & . & 4 & . & . & . & . & . \\
\hline & Knautia drymeia & E1 & 25 & 22 & . & 17 & . & . & . & . & . \\
\hline & Cardamine trifolia & E1 & . & 17 & . & 33 & . & . & . & 2 & . \\
\hline & Anemone trifolia & E1 & . & 6 & . & . & . & . & . & . & . \\
\hline & Stellaria montana & E1 & . & . & . & 21 & . & . & . & . & . \\
\hline & Euphorbia carniolica & E1 & . & . & . & 4 & . & . & . & . & . \\
\hline \multirow[t]{26}{*}{ FS } & Fagetalia sylvaticae & & & & & & & & & & \\
\hline & Daphne mezereum & E2a & 100 & 50 & 28 & 21 & . & 4 & 4 & 2 & . \\
\hline & Paris quadrifolia & E1 & 100 & 72 & 43 & 54 & 5 & 17 & . & . & . \\
\hline & Dryopteris filix-mas & E1 & 100 & 50 & 41 & 50 & 28 & 9 & 13 & . & . \\
\hline & Mercurialis perennis & E1 & 100 & 39 & 17 & 17 & . & . & . & . & . \\
\hline & Ranunculus lanuginosus & E1 & 100 & 22 & . & 25 & . & . & . & . & . \\
\hline & Lilium martagon & E1 & 75 & 28 & 9 & 8 & 3 & . & . & . & 17 \\
\hline & Symphytum tuberosum & E1 & 75 & 22 & . & 13 & . & . & . & . & . \\
\hline & Chrysosplenium alternifolium & E1 & 75 & 17 & 30 & 25 & 9 & 13 & 4 & . & . \\
\hline & Polystichum aculeatum & E1 & 75 & 11 & . & 13 & . & . & . & . & . \\
\hline & Myosotis sylvatica & E1 & 75 & 11 & 33 & 29 & 21 & 56 & 17 & 8 & . \\
\hline & Poa nemoralis & E1 & 50 & 28 & 17 & 4 & 10 & 48 & . & 9 & . \\
\hline & Lonicera alpigena & E2a & 50 & 22 & 15 & 8 & 1 & 13 & . & 2 & . \\
\hline & Epilobium montanum & E1 & 50 & . & 19 & 33 & 25 & 4 & . & 4 & . \\
\hline & Melica nutans & E1 & 25 & 22 & 7 & . & . & . & . & 4 & . \\
\hline & Adoxa moschatellina & E1 & 25 & 6 & . & 13 & . & . & . & . & . \\
\hline & Actaea spicata & E1 & 25 & 6 & 7 & 4 & 1 & . & . & . & . \\
\hline & Galeobdolon flavidum & E1 & . & 67 & 44 & 25 & 3 & . & . & 4 & . \\
\hline & Fagus sylvatica & E2 & . & 11 & 2 & 13 & 1 & . & . & . & . \\
\hline & Petasites albus & E1 & . & 6 & 6 & 8 & 17 & . & . & . & . \\
\hline & Lysimachia nemorum & E1 & . & . & 26 & . & 19 & . & . & . & . \\
\hline & Phyteuma spicatum & E1 & . & . & 20 & 4 & 6 & . & . & . & . \\
\hline & Prenanthes purpurea & E1 & . & . & 17 & 4 & 8 & . & . & 6 & . \\
\hline & Aruncus dioicus & E1 & . & . & 4 & 8 & 1 & . & . & . & . \\
\hline & Scrophularia nodosa & E1 & . & . & . & 13 & . & . & . & 2 & . \\
\hline & Galium laevigatum & E1 & . & . & . & 8 & . & . & . & . & . \\
\hline
\end{tabular}


Successive number (Zaporedna številka)

\begin{tabular}{|c|c|c|c|c|c|c|c|c|c|c|c|}
\hline & Successive number (Zaporedna številka) & & 1 & 2 & 3 & 4 & 5 & 6 & 7 & 8 & 9 \\
\hline & Carex sylvatica & E1 & . & . & . & 8 & . & . & . & . & . \\
\hline & Cardamine impatiens & E1 & . & . & . & 4 & . & . & . & . & . \\
\hline & Corydalis solida & E1 & . & . & . & 4 & . & . & . & . & . \\
\hline & Prunus avium & E3 & . & . & . & 4 & . & . & . & . & . \\
\hline & Leucojum vernum & E1 & . & . & . & 4 & . & . & . & . & . \\
\hline & Asarum europaeum & E1 & . & . & . & 4 & . & . & . & . & . \\
\hline & Polystichum braunii & E1 & . & . & . & 4 & 1 & . & . & . & . \\
\hline & Polystichum x luerssenii & E1 & . & . & . & 4 & . & . & . & . & . \\
\hline & Brachypodium sylvaticum & E1 & . & . & . & 4 & . & . & . & . & . \\
\hline & Impatiens noli-tangere & E1 & . & . & . & . & 2 & . & . & . & . \\
\hline & Dryopteris affinis & E1 & . & . & . & . & . & 4 & . & . & 33 \\
\hline & Luzula nivea & E1 & . & . & . & . & . & 9 & . & 6 & . \\
\hline & Equisetum sylvaticum & E1 & . & . & . & . & . & 4 & . & . & . \\
\hline & Geranium robertianum & E1 & . & . & . & . & . & . & . & 2 & . \\
\hline & Petasites albus & E1 & . & . & . & . & . & . & . & 2 & . \\
\hline \multirow[t]{5}{*}{ QP } & Quercetalia pubescentis & & & & & & & & & & \\
\hline & Sorbus aria & E3 & . & . & . & 17 & . & . & . & . & . \\
\hline & Sorbus aria & E2 & . & . & . & 8 & . & . & . & . & . \\
\hline & Convallaria majalis & E1 & . & . & . & 4 & . & . & . & 2 & . \\
\hline & Hypericum montanum & E1 & . & . & . & . & . & . & . & 4 & . \\
\hline \multirow[t]{17}{*}{$\mathrm{QF}$} & Querco-Fagetea & & & & & & & & & & \\
\hline & Anemone nemorosa & E1 & 25 & 22 & . & 63 & . & . & . & . & 83 \\
\hline & Hepatica nobilis & E1 & . & 33 & . & . & . & . & . & . & . \\
\hline & Alnus incana & E1 & . & . & 2 & . & . & . & . & . & . \\
\hline & Rubus caesius & E1 & . & . & 75 & . & . & . & . & . & . \\
\hline & Stellaria holostea & E1 & . & . & . & 13 & . & . & . & . & . \\
\hline & Hepatica nobilis & E1 & . & . & . & 8 & . & . & . & . & . \\
\hline & Ranunculus auricomus agg. & E1 & . & . & . & 8 & . & . & . & . & . \\
\hline & Aegopodium podagraria & E1 & . & . & . & 4 & . & . & . & . & . \\
\hline & Festuca heterophylla & E1 & . & . & . & 4 & . & . & . & 2 & . \\
\hline & Cruciata glabra & E1 & . & . & . & 4 & . & . & . & . & . \\
\hline & Viola riviniana & E1 & . & . & . & 4 & . & . & . & . & . \\
\hline & Listera ovata & E1 & . & . & . & . & 1 & . & . & . & . \\
\hline & Orchis mascula & E1 & . & . & . & . & . & 4 & . & . & . \\
\hline & Dactylorhiza fuchsii & E1 & . & . & . & . & . & . & . & 2 & . \\
\hline & Melampyrum pratense & E1 & . & . & . & . & . & . & . & . & 33 \\
\hline & Carex brizoides & E1 & . & . & . & . & . & . & . & . & 17 \\
\hline \multirow[t]{4}{*}{ TG } & Trifolio-Geranietea & & & & & & & & & & \\
\hline & Digitalis grandiflora & E1 & 50 & 6 & . & . & . & . & . & . & . \\
\hline & Laserpitium latifolium & E1 & . & . & . & 4 & . & . & . & . & . \\
\hline & Hypericum perforatum & E1 & . & . & . & . & . & . & . & 4 & . \\
\hline \multirow[t]{13}{*}{ ES } & Elyno-Seslerietea & & & & & & & & & & \\
\hline & Sesleria caerulea subsp. calcaria & E1 & . & 33 & 6 & 4 & . & . & . & . & . \\
\hline & Carex ferruginea & E1 & . & 22 & 24 & 4 & 8 & 9 & 8 & . & . \\
\hline & Alchemilla vulgaris s.str. & E1 & . & 22 & . & . & . & 22 & . & 8 & . \\
\hline & Astrantia bavarica & E1 & . & 17 & . & . & . & . & . & . & . \\
\hline & Laserpitium peucedanoides & E1 & . & 11 & . & . & . & . & . & . & . \\
\hline & Campanula witasekiana & E1 & . & 11 & . & . & . & . & . & . & . \\
\hline & Galium anisophyllum & E1 & . & 11 & 4 & . & . & . & 4 & 4 & . \\
\hline & Heliosperma alpestre & E1 & . & 11 & . & . & . & . & . & . & . \\
\hline & Scabiosa lucida subsp. stricta & E1 & . & 6 & 6 & . & 1 & . & . & . & . \\
\hline & Betonica alopecuros & E1 & . & 6 & 4 & 8 & . & . & . & . & . \\
\hline & Festuca calva & E1 & . & 6 & . & 4 & . & . & . & . & . \\
\hline & Leucanthemum adustum & E1 & . & 6 & . & . & . & 4 & . & . & . \\
\hline
\end{tabular}


Successive number (Zaporedna številka)

Koeleria eriostachya

Phleum hirsutum

Pulsatilla alpina subsp. austroalpina

Bartsia alpina

Carex sempervirens

Carex firma

Ranunculus montanus agg.

Aster bellidiastrum

Cardus defloratus

Polygonum viviparum

Phyteuma orbiculare

Luzula glabrata

Thesium alpinum

Heracleum austriacum

Acinos alpinus

Ligusticum mutellina

Thymus parecox

Bromopsis transsilvanica

Carduus crassifolius

Alchemilla alpina

Alchemilla fissa

Festuca pulchella

Myosotis alpestris

Gentiana lutea

Gentianella anisodonta

$\begin{array}{ll} & 1 \\ \text { E1 } & \cdot \\ \text { E1 } & \cdot \\ \text { E1 } & \cdot \\ \text { E1 } & \cdot \\ \text { E1 } & \cdot \\ \text { E1 } & \cdot \\ \text { E1 } & \cdot \\ \text { E1 } & \cdot \\ \text { E1 } & \cdot \\ \text { E1 } & \cdot \\ \text { E1 } & \cdot \\ \text { E1 } & \cdot \\ \text { E1 } & \cdot \\ \text { E1 } & \cdot \\ \text { E1 } & \cdot \\ \text { E1 } & \cdot \\ \text { E1 } & \cdot \\ \text { E1 } & \cdot \\ \text { E1 } & \cdot \\ \text { E1 } & \cdot \\ \text { E1 } & \cdot \\ \text { E1 } & \cdot \\ \text { E1 } & \cdot \\ \text { E1 } & \cdot \\ \text { E1 } & \cdot \\ \end{array}$

CU Calluno-Ulicetea

Coeloglossum viride

Potentilla erecta

Luzula multiflora

Geum montanum

Phleum alpinum agg.

Galium pumilum

Nardus stricta

Phyteuma zahlbruckneri

Alchemilla flabellata

Calluna vulgaris

Gentiana purpurea

Phyteuma betonicifolium

Carex pilulifera

Arnica montana

Campanula barbata

Omalotheca sylvatica

Festuca filiformis

E1

E1

E1

E1

E1

E1

E1

E1

E1

E1

E1

E1

E1

E1

E1

E1

E1

JT Juncetea trifidi

Soldanella alpina

Gentiana pannonica

Agrostis schraderiana (A. agrostiflora)

E1

E1

Gentiana punctata

Agrostis rupestris

Juncus trifidus

Leontodon helveticus

Soldanella pusilla

Avenula vesicolor

Luzula alpinopilosa

\begin{tabular}{|c|c|c|c|c|c|c|c|}
\hline 2 & 3 & 4 & 5 & 6 & 7 & 8 & 9 \\
\hline 6 & . & . & . & . & . & . & . \\
\hline 6 & . & . & . & . & . & . & . \\
\hline 6 & . & . & . & . & . & . & . \\
\hline 6 & . & . & . & . & . & . & . \\
\hline 6 & . & . & . & . & . & . & . \\
\hline 6 & . & . & . & . & . & . & . \\
\hline . & 24 & . & 7 & 4 & 4 & 2 & . \\
\hline . & 20 & . & 7 & 4 & 4 & . & 17 \\
\hline . & 7 & . & 3 & . & . & . & . \\
\hline . & 6 & . & 1 & . & 4 & 4 & . \\
\hline . & 4 & . & . & . & . & . & . \\
\hline . & 4 & . & . & . & . & . & . \\
\hline . & 4 & . & 1 & . & 4 & . & . \\
\hline . & 2 & . & . & . & . & . & . \\
\hline . & 2 & . & . & . & . & . & . \\
\hline . & 2 & . & 8 & 17 & 8 & 6 & . \\
\hline . & 2 & . & . & . & 13 & 2 & . \\
\hline . & . & 4 & . & . & . & $\cdot$ & . \\
\hline . & . & 4 & . & $\cdot$ & $\cdot$ & . & $\cdot$ \\
\hline . & . & . & . & 4 & . & 4 & . \\
\hline$\cdot$ & . & $\cdot$ & . & 4 & . & $\cdot$ & . \\
\hline$\cdot$ & $\cdot$ & $\cdot$ & . & 9 & . & . & . \\
\hline$\cdot$ & $\cdot$ & $\cdot$ & $\cdot$ & 4 & . & $\cdot$ & $\cdot$ \\
\hline . & $\cdot$ & $\cdot$ & $\cdot$ & $\cdot$ & $\cdot$ & 2 & . \\
\hline & ${ }^{\circ}$ & $\cdot$ & $\cdot$ & $\cdot$ & $\cdot$ & . & 17 \\
\hline
\end{tabular}

\begin{tabular}{|c|c|c|c|c|c|c|c|c|}
\hline - & 11 & . & . & . & . & . & . & . \\
\hline . & . & 19 & . & 15 & 4 & 17 & 11 & . \\
\hline . & . & 9 & . & . & . & 21 & . & . \\
\hline . & . & 9 & . & 8 & . & 29 & 13 & . \\
\hline . & . & 9 & . & 7 & 13 & 21 & 11 & . \\
\hline . & . & 6 & . & 2 & . & 4 & . & . \\
\hline . & . & 6 & . & 3 & . & 25 & 2 & . \\
\hline . & . & . & 4 & . & . & . & 2 & . \\
\hline . & . & . & 4 & . & . & . & . & . \\
\hline . & . & . & . & 3 & . & 21 & . & 33 \\
\hline . & . & . & . & . & 13 & . & 6 & . \\
\hline . & . & . & . & . & 4 & . & . & . \\
\hline . & . & . & . & . & . & . & 2 & 17 \\
\hline . & . & . & . & . & . & . & 4 & . \\
\hline . & . & . & . & . & . & . & 2 & 50 \\
\hline . & . & . & . & . & . & . & 4 & . \\
\hline . & . & . & . & . & . & . & . & 17 \\
\hline
\end{tabular}




\begin{tabular}{|c|c|c|c|c|c|c|c|c|c|c|c|}
\hline & Successive number (Zaporedna številka) & & 1 & 2 & 3 & 4 & 5 & 6 & 7 & 8 & 9 \\
\hline & Erigeron alpinus & E1 & . & . & . & . & . & . & . & 2 & . \\
\hline & Campanula alpina & E1 & . & . & . & . & . & . & . & . & 17 \\
\hline & Pulsatilla alpina subsp. alba & E1 & . & . & . & . & . & . & . & . & 17 \\
\hline \multirow[t]{5}{*}{ LV } & Loiseleurio-Vaccinietea & & & & & & & & & & \\
\hline & Astrantia minor & E1 & . & . & . & . & . & 4 & . & 13 & . \\
\hline & Vaccinium uliginosum & E1 & . & . & . & . & . & . & . & 13 & . \\
\hline & Vaccinium gaultherioides & E1 & . & . & . & . & . & . & . & . & 17 \\
\hline & Empetrum hermaphroditum & E1 & . & . & . & . & . & . & . & . & 17 \\
\hline \multirow[t]{12}{*}{$\mathrm{PaT}$} & Poo alpinae-Trisetalia & & & & & & & & & & \\
\hline & Agrostis capillaris & E1 & 4 & 6 & . & . & . & . & . & 8 & . \\
\hline & Trollius europaeus & E1 & . & 33 & 7 & 13 & 1 & 13 & . & 2 & . \\
\hline & Campanula scheuchzeri & E1 & . & 17 & 39 & . & 14 & . & 29 & 9 & . \\
\hline & Cardaminopsis halleri s. lat. & E1 & . & 11 & . & 29 & . & . & . & 2 & . \\
\hline & Poa alpina & E1 & . & 11 & 20 & 8 & 10 & 4 & 17 & 2 & . \\
\hline & Festuca nigrescens / Festuca rubra agg. & E1 & . & 6 & 9 & 8 & 6 & . & 33 & 15 & . \\
\hline & Cerastium fontanum & E1 & . & 6 & . & . & . & . & . & . & . \\
\hline & Astrantia major & E1 & . & . & 13 & . & . & . & . & . & . \\
\hline & Ranunculus nemorosus & E1 & . & . & 19 & . & 10 & . & 4 & . & . \\
\hline & Crocus albiflorus & E1 & . & . & . & 8 & . & . & . & . & . \\
\hline & Polygonum bistorta & E1 & . & . & . & . & . & 9 & . & . & . \\
\hline \multirow[t]{20}{*}{ MA } & Molinio-Arrhenatheretea & & & & & & & & & & \\
\hline & Deschampsia cespitosa & E1 & . & 28 & 46 & 63 & 45 & 35 & 63 & 28 & 50 \\
\hline & Angelica sylvestris & E1 & . & 17 & 2 & 17 & 5 & 9 & 4 & . & . \\
\hline & Dactylis glomerata & E1 & . & 11 & 13 & . & 6 & 4 & . & . & . \\
\hline & Caltha palustris & E1 & . & 6 & 9 & 8 & 6 & 9 & 17 & . & . \\
\hline & Prunella vulgaris & E1 & . & . & 25 & . & . & . & . & . & . \\
\hline & Veronica chamaedrys & E1 & . & . & 11 & . & 13 & . & . & 2 & . \\
\hline & Lotus corniculatus & E1 & . & . & 9 & . & 4 & . & 4 & . & . \\
\hline & Agrostis stolonifera & E1 & . & . & 7 & . & 21 & . & 4 & 2 & . \\
\hline & Leontodon hispidus & E1 & . & . & 7 & . & 2 & . & 17 & . & . \\
\hline & Dactylorhiza maculata & E1 & . & . & 6 & . & 3 & . & 4 & . & . \\
\hline & Ranunculus repens & E1 & . & . & 6 & . & 17 & . & 8 & . & . \\
\hline & Valeriana officinalis & E1 & . & . & 4 & . & 3 & . & . & . & . \\
\hline & Trifolium pratense & E1 & . & . & 4 & . & 6 & . & 8 & . & . \\
\hline & Achillea millefolium & E1 & . & . & 2 & . & 13 & . & 4 & 4 & . \\
\hline & Galium mollugo & E1 & . & . & . & 8 & . & . & . & . & . \\
\hline & Astrantia major & E1 & . & . & . & 4 & . & . & . & . & . \\
\hline & Agrostis gigantea (A. alba) & E1 & . & . & . & . & . & 4 & . & . & . \\
\hline & Ranunculus aconitifolius & E1 & . & . & . & . & . & . & . & 8 & . \\
\hline & Cirsium eriophorum & E1 & . & . & . & . & . & . & . & 2 & . \\
\hline \multirow[t]{3}{*}{ SCf } & Scheuchzerio-Caricetea fuscae & & & & & & & & & & \\
\hline & Parnassia palustris & E1 & . & 28 & 20 & . & 5 & . & . & . & . \\
\hline & Tofieldia calyculata & E1 & . & . & 4 & . & 1 & . & . & . & . \\
\hline \multirow[t]{2}{*}{ FB } & Festuco-Brometea & & & & & & & & & & \\
\hline & Briza media & E1 & . & . & 4 & . & 1 & . & . & . & . \\
\hline \multirow[t]{4}{*}{ EA } & Epilobietea angustifolii & & & & & & & & & & \\
\hline & Tussilago farfara & E1 & . & . & 7 & . & 12 & 13 & 17 & . & . \\
\hline & Chamaenerion angustifolium & E1 & . & . & . & 8 & . & 4 & . & 8 & 17 \\
\hline & Calamagrostis epigejos & E1 & . & . & . & . & . & . & . & 2 & . \\
\hline \multirow[t]{5}{*}{ TR } & Thlaspietea rotundifolii & & & & & & & & & & \\
\hline & Valeriana montana & E1 & 75 & . & 22 & . & 4 & . & . & . & . \\
\hline & Festuca nitida & E1 & . & 50 & . & 4 & . & . & . & . & . \\
\hline & Rhodiola rosea & E1 & . & 33 & . & . & . & . & . & 8 & . \\
\hline & Dryopteris villarii & E1 & . & 17 & . & . & . & . & . & . & . \\
\hline
\end{tabular}


Successive number (Zaporedna številka)

Arabis alpina

Astrantia carniolica

Aquilegia einseleana

Gymnocarpium robertianum

Biscutela laevigata

Rumex scutatus

Ligusticum segueri

Geranium macrorrhizum

Cardamine resedifolia

Campanula cochleariifolia

Hieracium bifidum

AT Asplenietea trichomanis

Cystopteris fragilis

Paederota lutea

Cystopteris regia

Heliosperma pusillum

Valeriana saxatilis

Moehringia muscosa

Asplenium ruta-muraria

Hieracium schmidtii

MC Montio-Cardaminetea

$\begin{array}{ll} & 1 \\ \text { E1 } & \cdot \\ \text { E1 } & \cdot \\ \text { E1 } & \cdot \\ \text { E1 } & \cdot \\ \text { E1 } & \cdot \\ \text { E1 } & \cdot \\ \text { E1 } & \cdot \\ \text { E1 } & \cdot \\ \text { E1 } & \cdot \\ \text { E1 } & \cdot \\ \text { E1 } & \cdot\end{array}$

$\begin{array}{cccccccc}2 & 3 & 4 & 5 & 6 & 7 & 8 & 9 \\ 11 & 17 & 8 & 4 & 4 & . & . & .\end{array}$

\begin{tabular}{|c|c|}
\hline E1 & 33 \\
\hline E1 & 28 \\
\hline E1 & 11 \\
\hline E1 & 6 \\
\hline E1 & 6 \\
\hline E1 & 6 \\
\hline
\end{tabular}

Saxifraga stellaris s. lat.

Cardamine flexuosa

Cardamine amara

Other species (Druge vrste)

Alchemilla sp.

Salix eleagnos

Mentha longifolia

Betula pubescens

Betula pendula

Galeopsis tetrahit

Petasites hybridus

Salix myrsinifolia

E1

E1

E1

E1

E1

E1

E2

E2

E1

E1

E2

ML Mosses and lichens (Mahovi in lišaji)

Ctenidium molluscum

Rhytidiadelphus triquetrus

Tortella tortuosa

Hylocomium splendens

Pseudoleskeella catenulata

Dicranum scoparium

Conocephalum conicum

Polytrichum formosum

Fissidens dubius

Eurhynchium angustirete

Peltigera leucophlebia

Plagiochila porelloides

Marchantia polymorpha

Plagiochila asplenioides

Rhizomnium punctatum

Mnium sp.

Anomodon sp.

Orthothecium rufescens

Peltigera canina

Hylocomium splendens

$\begin{array}{lccccccccc}\text { E0 } & . & 78 & 19 & 29 & 1 & . & 4 & . & . \\ \text { E0 } & . & 61 & 15 & 8 & 6 & . & 13 & . & 67 \\ \text { E0 } & . & 44 & . & 13 & . & . & . & . & 50 \\ \text { E0 } & . & 33 & 9 & . & . & . & . & . & . \\ \text { E0 } & . & 28 & . & . & . & . & . & . & . \\ \text { E0 } & . & 28 & 17 & . & 12 & 4 & 13 & . & 83 \\ \text { E0 } & . & 28 & . & . & . & 9 & . & . & . \\ \text { E0 } & . & 22 & 15 & 25 & 16 & 4 & 25 & . & 83 \\ \text { E0 } & . & 22 & . & 4 & . & . & . & . & . \\ \text { E0 } & . & 22 & . & . & . & . & . & . & . \\ \text { E0 } & . & 16,7 & . & . & . & . & . & . & . \\ \text { E0 } & . & 17 & . & 8 & . & . & . & . & . \\ \text { E0 } & . & 11 & . & 4 & . & 9 & . & . & . \\ \text { E0 } & . & 11 & 9 & 4 & 8 & 9 & . & . & . \\ \text { E0 } & . & 6 & 7 & . & 23 & 9 & 4 & . & 17 \\ \text { E0 } & . & 6 & . & 4 & . & 34 & . & . & . \\ \text { E0 } & . & 6 & . & . & . & . & . & . & . \\ \text { E0 } & . & 6 & . & . & . & . & . & . & . \\ \text { E0 } & . & 6 & . & 4 & . & . & . & . & . \\ \text { E0 } & . & 6 & 9 & . & 8 & . & 21 & . & 33\end{array}$ 
Successive number (Zaporedna številka)

Sphagnum subnites

Plagiomnium rostratum

Drepanocladus uncinatus

Pleurozium schrberi

Atrichum undulatum

Isothecium alopecuroides

Plagiothecium undulatum

Brachythecium plumosum

Homalothecium lutescens

Pellia endiviifolia

Cirriphyllum crassinervum

Conocephalum conicum

Hookeria lucens

Fissidens taxifolius

Atrichum angustatum

Cephalozia bicuspidata

Herzogiella seligeri

Plagithecium nemorale

Calypogea azurea

Chiloscyphus polyanthos

Riccardia palmata

Campylium stellatum

Polytrichum commune

Climacium dendroides

Polytrichum alpinum

Brachythecium selebrosum

Plagiothecium denticulatum

Brachythecium reflaxum

Brachythecium starkei

Diplophyllum albicans

Pogonatum unigerum

Polytrichum juniperinum

Dicranum polysetum

Cetraria islandica

Rhizocarpon geographicum

Cephalozia lacinulata

Calypogeia azurea

Nardia scalaris

Amphidium mougeotii

Hypnum cupressiforme var. cupressiforme

Calypogeia integristipula

Tortella densa

Racomitrium lanuginosum

Brachytehecium rutabulum

\begin{tabular}{|c|c|c|c|c|c|c|c|c|c|}
\hline & 1 & 2 & 3 & 4 & 5 & 6 & 7 & 8 & 9 \\
\hline E0 & . & 6 & . & . & . & . & . & . & 33 \\
\hline E0 & . & 6 & . & 4 & . & . & . & . & . \\
\hline E0 & . & . & 7 & . & 7 & . & . & . & . \\
\hline E0 & . & . & 6 & . & 4 & 4 & 21 & . & . \\
\hline E0 & . & . & . & 8 & . & . & . & . & . \\
\hline E0 & . & . & . & 8 & . & . & . & . & . \\
\hline E0 & . & . & . & 4 & . & . & . & . & 17 \\
\hline E0 & . & . & . & 4 & . & . & . & . & 17 \\
\hline E0 & . & . & . & 4 & . & . & . & . & . \\
\hline E0 & . & . & . & 8 & . & . & . & . & . \\
\hline E0 & . & . & . & 8 & . & . & . & . & . \\
\hline E0 & . & . & . & 4 & . & . & . & . & . \\
\hline E0 & . & . & . & 4 & . & . & . & . & . \\
\hline E0 & . & . & . & 4 & . & . & . & . & . \\
\hline E0 & . & . & . & 4 & . & . & . & . & . \\
\hline E0 & . & . & . & 4 & . & . & . & . & . \\
\hline E0 & . & . & . & 4 & . & . & . & . & . \\
\hline E0 & . & . & . & 4 & . & . & . & . & . \\
\hline E0 & . & . & . & 4 & . & . & . & . & . \\
\hline E0 & . & . & . & 4 & . & . & . & . & . \\
\hline E0 & . & . & . & 4 & . & . & . & . & . \\
\hline E0 & . & . & . & 4 & . & . & . & . & . \\
\hline E0 & . & . & . & . & 10 & . & 25 & . & . \\
\hline E0 & . & . & . & . & 1 & . & . & . & . \\
\hline E0 & . & . & . & . & . & 17 & . & . & 17 \\
\hline E0 & . & . & . & . & . & 17 & . & . & . \\
\hline E0 & . & . & . & . & . & 17 & . & . & . \\
\hline E0 & . & . & . & . & . & 13 & . & . & . \\
\hline E0 & . & . & . & . & . & 9 & . & . & . \\
\hline E0 & . & . & . & . & . & . & . & . & 50 \\
\hline E0 & . & . & . & . & . & . & . & . & 50 \\
\hline E0 & . & . & . & . & . & . & . & . & 17 \\
\hline E0 & . & . & . & . & . & . & . & . & 33 \\
\hline E0 & . & . & . & . & . & . & . & . & 33 \\
\hline E0 & . & . & . & . & . & . & . & . & 17 \\
\hline E0 & . & . & . & . & . & . & . & . & 17 \\
\hline E0 & . & . & . & . & . & . & . & . & 17 \\
\hline E0 & . & . & . & . & . & . & . & . & 17 \\
\hline E0 & . & . & . & . & . & . & . & . & 17 \\
\hline E0 & . & . & . & . & . & . & . & . & 17 \\
\hline E0 & . & . & . & . & . & . & . & . & 17 \\
\hline E0 & . & . & . & . & . & . & . & . & 17 \\
\hline E0 & . & . & . & . & . & . & . & . & 17 \\
\hline E0 & . & . & . & . & . & . & . & . & 17 \\
\hline
\end{tabular}

1 Rhododendro hirsuti-Alnetum viridis ass. nova hoc loco, Aichinger (1933), the Karavanke range, Austria

2 Rhododendro hirsuti-Alnetum viridis ass. nova, hoc loco, the Julian Alps, the Karavanke range, Slovenia, limestone, dolomite

3 Rhododendro hirsuti-Alnetum viridis ass. nova hoc loco = Alnetum viridis aceretosum pseudoplatani prov., Karner (2007), Austria

4 Rhododendro hirsuti-Alnetum viridis, Alnetum viridis s. lat. Slovenia

5 Alnetum viridis typicum, Karner (2007), Austria

6 Alnetum viridis, Braun-Blanquet (1973), Switzerland

7 Alnetum viridis rhododendretosum ferruginei prov., Karner (2007), Austria

8 Rhododendro ferruginei-Alnetum viridis Boscutti, Poldini \& Buccheri 2013, Alps

9 Huperzio selagi-Alnetum viridis ass. nova hoc loco, Kamen, the Savinja Alps, Slovenia 
Table 5: Phytosociological groups in the communities with dominant Alnus viridis in the Alps (relative frequencies)

Tabela 5: Fitocenološke skupine v združbah s prevladujočo vrsto Alnus viridis v Alpah (relativne frekvence)

Successive number (Zaporedna številka)

Number of relevés (Število popisov)

Sign for the syntaxa (Oznaka sintaksonov)

Betulo-Alnetea viridis

Mulgedio-Aconitetea

Sambuco-Salicion capreae

Vaccinio-Piceetea

Erico-Pinetea

Aremonio-Fagion

Fagetalia sylvaticae

Quercetalia pubescentis, Querco-Fagetea

Elyno-Seslerietea

Calluno-Ulicetea

Juncetea trifidi, Loiseleurio-Vaccinietea

Poo alpinae-Trisetalia

Molinio-Arrhenatheretea

Trifolio-Geranietea, Epilobietea angustifolii

Thlaspietea rotundifolii

Asplenietea trichomanis

Montio-Cardaminetea, Scheuchzerio-Caricetea

Other species (Druge vrste)

Mosses and lichens (Mahovi in lišaji)

$\begin{array}{ccccccccc}1 & 2 & 3 & 4 & 5 & 6 & 7 & 8 & 9 \\ 4 & 18 & 54 & 24 & 107 & 23 & 24 & 53 & 6\end{array}$
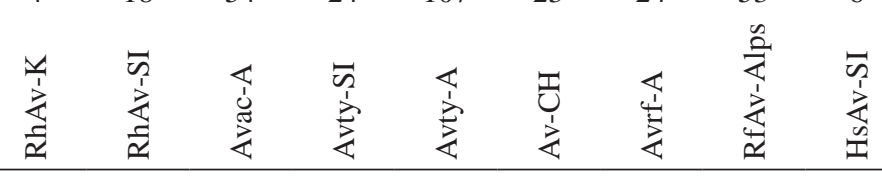

$\begin{array}{lllllllll}40 & 29 & 34 & 29 & 37 & \mathbf{4 8} & 25 & 26 & 6.1\end{array}$

$\begin{array}{lllllllll}3.4 & 2.1 & 3.8 & \mathbf{6 . 8} & 4.8 & 3.2 & 2.4 & \mathbf{7} & \mathbf{6 . 1}\end{array}$

$\begin{array}{lllllllll}17 & 23 & 18 & 22 & 22 & 19 & 33 & 37 & 39\end{array}$

$\begin{array}{lllllllll}1.7 & 4.4 & 2.7 & 1 & 0.8 & 0.3 & 1.2 & 1.3 & 3.7\end{array}$

$\begin{array}{lllllllll}3.4 & 2.5 & 0.7 & 2.9 & 0 & 0 & 0 & 0.1 & 0\end{array}$

$\begin{array}{lllllllll}26 & 11 & 12 & 16 & 7.4 & \mathbf{6 . 9} & 1.5 & 3.2 & 1.2\end{array}$

$\begin{array}{lllllllll}0.6 & 1 & 2 & \mathbf{4} & 0 & 0.1 & 0 & 0.6 & \mathbf{4 . 9}\end{array}$

$\begin{array}{lllllllll}0 & 3.4 & 3.2 & 0.8 & 1.6 & 3 & 2 & 1.9 & 0.8\end{array}$

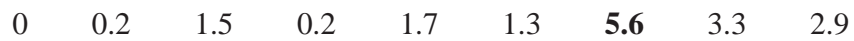

$\begin{array}{lllllllll}0 & 1.1 & 2.0 & 0 & 1.6 & 1.5 & \mathbf{7 . 0} & \mathbf{5 . 8} & \mathbf{4 . 9}\end{array}$

$\begin{array}{lllllllll}0.1 & 1.6 & 2.8 & 1.9 & 1.8 & 1 & 3.4 & 2.2 & 0\end{array}$

$\begin{array}{lllllllll}0 & 1.1 & 4 & 2.9 & \mathbf{6 . 3} & 2.3 & 5.4 & 2.7 & 0\end{array}$

$\begin{array}{lllllllll}1.1 & 0.1 & 0.2 & 0.4 & 0.5 & 0.6 & 0.7 & 0.8 & 0.4\end{array}$

$\begin{array}{lllllllll}1.7 & 4.1 & 1.8 & 1.3 & 0.5 & 0.6 & 0.2 & 0.9 & 2\end{array}$

$\begin{array}{lllllllll}0 & 2.9 & 0.8 & 0.5 & 0 & 0.3 & 0 & 0.2 & 1.2\end{array}$

$\begin{array}{lllllllll}0 & 0.5 & 0.7 & 0.1 & 1.9 & 0.5 & 1.4 & 0.2 & 1.2\end{array}$

$\begin{array}{lllllllll}0 & 0 & 1.6 & 0.4 & 2.2 & 1.1 & 1.5 & 0.3 & 1.6\end{array}$

$\begin{array}{rrrrrrrrr}0 & 8.3 & 3 & 5.6 & 4.2 & 5.8 & 5.1 & 0 & \mathbf{2 0} \\ 100 & 100 & 100 & 100 & 100 & 100 & 100 & 100 & 100\end{array}$


Table 6: Alno viridis-Sorbetum aucupariae ass. nov.

Tabela 6: Alno viridis-Sorbetum aucupariae ass. nov.

\begin{tabular}{|c|c|c|c|c|c|c|c|c|c|c|c|c|}
\hline Number of relevé (Zaporedna številka popisa) & & 1 & 2 & 3 & 4 & 5 & 6 & 7 & 8 & 9 & 10 & \\
\hline Database number of relevé (Delovna številka popisa) & & 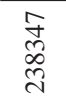 & 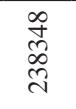 & 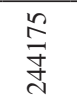 & $\begin{array}{l}\stackrel{J}{N} \\
\text { 岁 } \\
\text { ন }\end{array}$ & $\begin{array}{l}\infty \\
\stackrel{9}{\sim} \\
\stackrel{W}{\sim}\end{array}$ & $\begin{array}{l}\text { 竎 } \\
\text { 岁 } \\
\text { V }\end{array}$ & $\begin{array}{l}\text { 寺 } \\
\stackrel{\mathfrak{f}}{\sim}\end{array}$ & $\begin{array}{l}\stackrel{9}{9} \\
\stackrel{\text { ஸे }}{\sim}\end{array}$ & 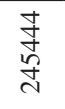 & 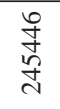 & \\
\hline Elevation in m (Nadmorska višina v m) & & $\begin{array}{l}\stackrel{\text { }}{-} \\
-\end{array}$ & $\underset{\sim}{\stackrel{+}{+}}$ & $\begin{array}{l}\infty \\
\stackrel{o}{\beth}\end{array}$ & $\underset{\sim}{\stackrel{\overbrace{}}{ত}}$ & $\stackrel{\text { 孚 }}{二}$ & $\begin{array}{l}\text { กิ } \\
\text { 금 }\end{array}$ & $\begin{array}{l}\text { लू } \\
\text { [n }\end{array}$ & 오 & 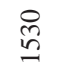 & $\begin{array}{l}\stackrel{8}{\circ} \\
\stackrel{\leftrightarrow}{n}\end{array}$ & \\
\hline Aspect (Lega) & & NE & NE & $\mathrm{NE}$ & $\mathrm{N}$ & NE & $\mathrm{N}$ & $\mathrm{N}$ & $\mathrm{N}$ & $\mathrm{N}$ & $\mathrm{N}$ & \\
\hline Slope in degrees (Nagib v stopinjah) & & 30 & 30 & 10 & 20 & 30 & 30 & 25 & 25 & 30 & 30 & \\
\hline Parent material (Matična podlaga) & & $\mathrm{R}$ & ALR & AGR & GR & AL & $\mathrm{AL}$ & $\mathrm{AL}$ & $\mathrm{AL}$ & $\mathrm{AL}$ & $\mathrm{AL}$ & \\
\hline Soil (Tla) & & Dy & Dy & Dy & Re & $\mathrm{K}$ & $\operatorname{Re}$ & $\operatorname{Re}$ & $\mathrm{K}$ & $\operatorname{Re}$ & $\mathrm{K}$ & \\
\hline Stoniness in \% (Kamnitost v \%) & & 0 & 0 & 10 & 20 & 30 & 35 & 40 & 25 & 30 & 20 & \\
\hline Cover of tree layer in \% (Zastiranje drevesne plasti v \%) & E3 & 80 & 70 & 100 & 80 & 70 & 70 & 70 & 70 & 80 & 70 & \\
\hline Cover of shrub layer in \% (Zastiranje grmovne plasti v \%): & E2 & 30 & 30 & 20 & 50 & 40 & 50 & 50 & 40 & 40 & 40 & \\
\hline Cover of herb layer in \% (Zastiranje zeliščne plasti v \%): & E1 & 90 & 90 & 70 & 70 & 80 & 90 & 90 & 80 & 90 & 90 & \\
\hline Cover of moss layer in \% (Zastiranje mahovne plasti v \%) & E0 & 5 & 5 & 5 & 20 & 5 & 5 & 5 & 5 & 5 & 5 & \\
\hline Number of species (Število vrst) & & 39 & 33 & 25 & 84 & 81 & 64 & 62 & 72 & 41 & 47 & \\
\hline Relevé area (Velikost popisne ploskve) & $\mathrm{m}^{2}$ & 400 & 200 & 100 & 200 & 200 & 200 & 200 & 200 & 200 & 200 & \\
\hline Date of taking relevé (Datum popisa) & & $\begin{array}{l}\stackrel{0}{\circ} \\
\stackrel{1}{N} \\
\stackrel{0}{N}\end{array}$ & 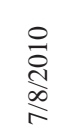 & 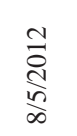 & 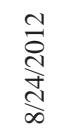 & 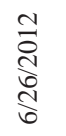 & 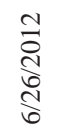 & 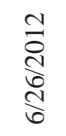 & 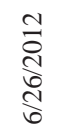 & 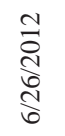 & 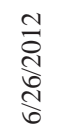 & \\
\hline Locality (Nahajališče) & & 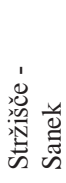 & 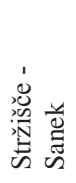 & $\begin{array}{l}\frac{0}{2} \\
\frac{0}{2} \\
\frac{1}{1} \\
\frac{\pi}{2} \\
\frac{1}{10}\end{array}$ & 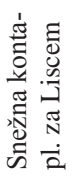 & $\frac{\Xi}{\stackrel{\Xi}{\pi}}$ & $\begin{array}{l}\text { 营 } \\
\text { 荧 }\end{array}$ & 营 & $\begin{array}{l}\text { 营 } \\
\text { 荧 }\end{array}$ & 营 & 㓂 & \\
\hline Quadrant (Kvadrant) & & $\begin{array}{l}\stackrel{+}{\sigma} \\
\text { 合 }\end{array}$ & $\frac{\stackrel{+}{\sigma}}{\stackrel{+}{\sigma}}$ & $\begin{array}{l}\frac{⿱}{S} \\
\stackrel{⿱}{\sigma}\end{array}$ & $\begin{array}{l}\stackrel{+}{\sigma} \\
\stackrel{+}{\sigma}\end{array}$ & 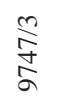 & 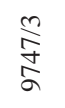 & 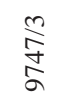 & 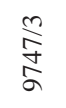 & $\frac{m}{\stackrel{N}{\sigma}}$ & $\underset{\frac{N}{N}}{\stackrel{m}{\sigma}}$ & \\
\hline Coordinate GK Y (D-48) & $\mathrm{m}$ & $\begin{array}{l}\mathscr{D} \\
0 \\
o \\
\forall \\
\forall\end{array}$ & 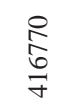 & 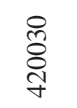 & 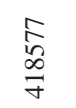 & 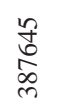 & $\begin{array}{l}\vec{\infty} \\
\underset{N}{\infty} \\
\stackrel{n}{\infty}\end{array}$ & 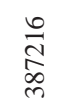 & 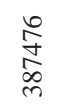 & 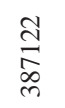 & $\begin{array}{l}\hat{\text { }} \\
\stackrel{2}{\infty} \\
\text { o }\end{array}$ & \\
\hline Coordinate GK X (D-48) & $\mathrm{m}$ & $\begin{array}{l}\infty \\
\stackrel{M}{0} \\
\stackrel{ِ}{\sim}\end{array}$ & $\begin{array}{l}\text { 금 } \\
\text { 옥 }\end{array}$ & $\begin{array}{l}\text { જે } \\
\text { త్ }\end{array}$ & 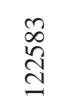 & $\begin{array}{l}\infty \\
\infty \\
\infty \\
\stackrel{0}{ } \\
=\end{array}$ & 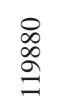 & $\begin{array}{l}\stackrel{+}{ } \\
\stackrel{0}{ } \\
\stackrel{\Xi}{=}\end{array}$ & 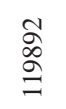 & $\begin{array}{l}\overrightarrow{7} \\
\text { o } \\
\stackrel{\Xi}{=}\end{array}$ & 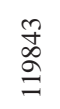 & \\
\hline Author of the relevé (Avtor popisa) & & ID & ID & ID & ID & ID & ID & ID & ID & ID & ID & $\operatorname{Pr}$ \\
\hline
\end{tabular}

Diagnostic species of the association (Diagnostične vrste asociacije)

SSC Sorbus aucuparia

SSC Sorbus aucuparia

SSC Sorbus aucuparia

SSC Sorbus aucuparia

MuA Veratrum album subsp. lobelianum

BA Alnus viridis

BA Alnus viridis

MuA Senecio cacaliaster

BA Salix appendiculata

$\begin{array}{lllllllllllrr}\text { E3 } & 5 & 4 & 5 & 4 & 4 & 4 & 4 & 4 & 4 & 4 & 10 & 100 \\ \text { E2b } & . & . & 1 & . & . & 1 & . & 1 & . & . & 3 & 30 \\ \text { E2a } & + & + & + & 1 & + & + & . & . & + & . & 7 & 70 \\ \text { E1 } & + & . & . & + & . & . & + & . & . & . & 3 & 30 \\ \text { E1 } & 2 & 2 & + & 1 & 1 & 1 & + & 1 & + & 1 & 10 & 100 \\ \text { E2b } & 1 & 1 & 1 & . & 2 & 1 & 1 & 1 & 1 & 1 & 9 & 90 \\ \text { E1 } & + & + & . & . & . & . & . & . & . & . & 2 & 20 \\ \text { E1 } & + & + & . & + & 1 & 1 & 1 & + & 1 & 1 & 9 & 90 \\ \text { E2b } & . & . & + & 3 & 2 & 3 & 3 & 1 & 2 & 3 & 8 & 80 \\ \text { E2a } & . & . & + & . & + & 1 & 1 & + & . & . & 5 & 50\end{array}$

BA Salix appendiculata

Differential species of lower units (Razlikovalne vrste nižjih enot)

VP Luzula sylvatica

BA Sorbus chamaemespilus

BA Sorbus chamaemespilus

TR Adenostyles glabra

AT Asplenium viride

AT Cystopteris fragilis

JT Soldanella alpina

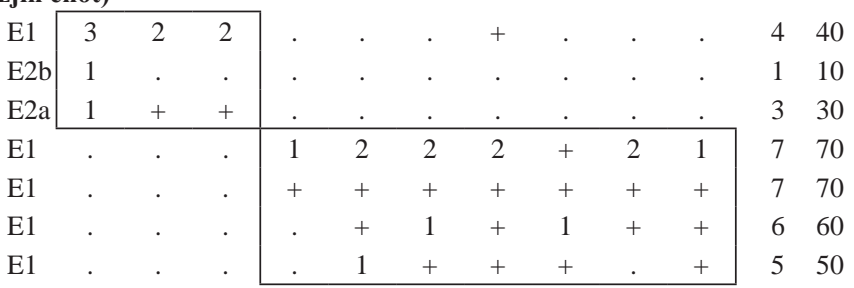


Number of relevé (Zaporedna številka popisa)

$\begin{array}{llllllllllll}1 & 2 & 3 & 4 & 5 & 6 & 7 & 8 & 9 & 10 & \text { Pr. } & \text { Fr. }\end{array}$

BA Betulo carpaticae-Alnetea viridis

Salix waldsteiniana

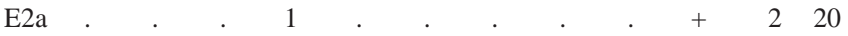

MuA Mulgedio-Aconitetea

Athyrium filix-femina

Polygonatum verticillatum

Ranunculus platanifolius

Geum rivale

Saxifraga rotundifolia

Thalictrum aquilegiifolium

Aconitum lycoctonum subsp. ranunculifolium

Geranium sylvaticum

Rumex arifolius

Alchemilla xanthochlora

Chaerophyllum hirsutum

Viola biflora

Adenostyles alliariae

Hypericum maculatum

Pleurospermum austriacum

Heracleum sphondylium subsp. montanum

Phyteuma ovatum

Myrrhis odorata

Streptopus amplexifolius

Athyrium distentifolium

Stellaria nemorum s.str.

Doronicum austriacum

Lathyrus occidentalis var. montanus

Allium victorialis

Milium effusum

Aconitum degenii subsp. paniculatum

Agropyron caninum

Crepis paludosa

Poa hybrida

Senecio ovatus

Senecio rivularis

Silene dioica

Chaerophyllum aureum

Tanacetum corymbosum subsp. clusii

\begin{tabular}{|c|c|c|c|c|c|c|c|c|c|c|c|c|}
\hline E1 & 1 & 2 & 3 & + & 2 & 3 & 3 & 3 & 3 & 4 & 10 & 100 \\
\hline E1 & + & + & 1 & + & 1 & + & + & 1 & . & + & 9 & 90 \\
\hline E1 & + & . & . & 1 & . & 1 & 1 & 1 & + & + & 7 & 70 \\
\hline E1 & . & . & . & 1 & 1 & 1 & 1 & 2 & 2 & 2 & 7 & 70 \\
\hline E1 & . & . & + & 1 & & + & . & 1 & 1 & + & 6 & 60 \\
\hline E1 & . & . & . & 1 & 1 & 1 & + & 2 & + & + & 7 & 70 \\
\hline E1 & . & . & . & 1 & 1 & 1 & 1 & + & + & . & 6 & 60 \\
\hline E1 & . & . & . & . & + & + & + & 1 & 1 & + & 6 & 60 \\
\hline E1 & . & . & 2 & + & + & + & . & 1 & . & . & 5 & 50 \\
\hline E1 & . & . & . & + & + & + & + & + & . & . & 5 & 50 \\
\hline E1 & . & . & . & + & 1 & + & + & 1 & . & . & 5 & 50 \\
\hline E1 & . & . & . & 1 & . & . & + & + & + & + & 5 & 50 \\
\hline E1 & . & . & + & 1 & . & . & . & 1 & . & 1 & 4 & 40 \\
\hline E1 & . & . & . & + & 1 & + & . & + & . & . & 4 & 40 \\
\hline E1 & . & . & . & . & . & + & + & . & 1 & + & 4 & 40 \\
\hline E1 & . & . & . & + & . & . & . & + & + & . & 3 & 30 \\
\hline E1 & . & . & . & . & 1 & . & + & . & . & + & 3 & 30 \\
\hline E1 & . & . & . & . & . & 2 & 3 & + & . & . & 3 & 30 \\
\hline E1 & . & . & 1 & + & . & . & . & . & . & . & 2 & 20 \\
\hline E1 & . & . & + & + & . & . & . & . & . & . & 2 & 20 \\
\hline E1 & . & . & 2 & . & + & . & . & . & . & . & 2 & 20 \\
\hline E1 & . & . & . & + & . & . & + & . & . & . & 2 & 20 \\
\hline E1 & . & . & . & . & . & . & + & . & + & . & 2 & 20 \\
\hline E1 & . & + & . & . & . & . & . & . & . & . & 1 & 10 \\
\hline E1 & . & . & 1 & . & . & . & . & . & . & . & 1 & 10 \\
\hline E1 & . & . & . & + & . & . & . & . & . & . & 1 & 10 \\
\hline E1 & . & . & . & + & . & . & . & . & . & . & 1 & 10 \\
\hline E1 & . & . & . & + & . & . & . & . & . & . & 1 & 10 \\
\hline E1 & . & . & . & + & . & . & . & . & . & . & 1 & 10 \\
\hline E1 & . & . & . & 1 & . & . & . & . & . & . & 1 & 10 \\
\hline E1 & . & . & . & + & . & . & . & . & . & . & 1 & 10 \\
\hline E1 & . & . & . & + & . & . & . & . & . & . & 1 & 10 \\
\hline E1 & . & . & . & . & + & . & . & . & . & . & 1 & 10 \\
\hline E1 & . & . & . & . & . & + & . & . & . & . & 1 & 10 \\
\hline E2a & + & 1 & 3 & 1 & 2 & 2 & 1 & 3 & 2 & 2 & 10 & 100 \\
\hline E1 & . & . & . & . & + & . & + & + & . & . & 3 & 30 \\
\hline E1 & . & . & . & + & . & . & . & + & . & . & 2 & 20 \\
\hline E3 & . & $r$ & . & . & . & . & . & . & . & . & 1 & 10 \\
\hline E2b & . & + & . & . & . & . & . & . & . & . & 1 & 10 \\
\hline
\end{tabular}

VP Vaccinio-Piceetea

Calamagrostis arundinacea

Gentiana asclepiadea

Oxalis acetosella

Rosa pendulina

Dryopteris dilatata

Solidago virgaurea

Valeriana tripteris

Maianthemum bifolium

Vaccinium myrtillus

Phegopteris connectilis

$\begin{array}{lllllllllllrr}\text { E1 } & 4 & 3 & + & + & 2 & 2 & 3 & 1 & 4 & 3 & 10 & 100 \\ \text { E1 } & 1 & 2 & . & 1 & 1 & 1 & + & 1 & . & + & 8 & 80 \\ \text { E1 } & + & . & 2 & 1 & + & 1 & . & 1 & 1 & 1 & 8 & 80 \\ \text { E2a } & + & + & . & + & + & . & + & + & 1 & + & 8 & 80 \\ \text { E1 } & 3 & 2 & 2 & 1 & . & . & . & + & + & + & 7 & 70 \\ \text { E1 } & . & . & + & 1 & + & + & + & + & + & . & 7 & 70 \\ \text { E1 } & . & . & . & 1 & 2 & + & 1 & 1 & + & . & 6 & 60 \\ \text { E1 } & 1 & 1 & . & + & + & . & . & . & . & . & 4 & 40 \\ \text { E1 } & 1 & 2 & . & + & . & . & . & + & . & . & 4 & 40 \\ \text { E1 } & 1 & 1 & . & + & . & + & . & . & . & . & 4 & 40\end{array}$


Number of relevé (Zaporedna številka popisa)

Polystichum lonchitis

Luzula luzuloides subsp. luzuloides

Picea abies

Picea abies

Picea abies

Picea abies

Blechnum spicant

Veronica urticifolia

Dryopteris expansa

Thelypteris limbosperma

Luzula luzuloides subsp. rubella

Luzula luzulina

Abies alba

Homogyne alpina

Lonicera caerulea

Pyrola minor

Saxifraga cuneifolia

Gymnocarpium dryopteris

Homogyne sylvestris

EP Erico-Pinetea

Rubus saxatilis

Cirsium erisithales

Rhododendron hirsutum

Carex ornithopoda

Molinia caerulea subsp. arundinacea

Pinus mugo

Aquilegia nigricans

AF Aremonio-Fagion

Cardamine trifolia

Knautia drymeia

Rhamnus fallax

Dentaria enneaphyllos

TA Tilio-Acerion

Acer pseudoplatanus

Acer pseudoplatanus

Acer pseudoplatanus

Polystichum braunii

Hesperis candida

Lunaria rediviva

Adoxa moschatellina

FS Fagetalia sylvaticae

Paris quadrifolia

Dryopteris filix-mas

Galium laevigatum

Lilium martagon

Symphytum tuberosum

Polystichum aculeatum

Fagus sylvatica

Fagus sylvatica

Fagus sylvatica

Fagus sylvatica

Laburnum alpinum

Laburnum alpinum

Laburnum alpinum

\begin{tabular}{|c|c|c|c|c|c|c|c|c|c|c|c|c|}
\hline & 1 & 2 & 3 & 4 & 5 & 6 & 7 & 8 & 9 & 10 & Pr. & Fr. \\
\hline E1 & . & . & . & . & $r$ & . & + & . & + & + & 4 & 40 \\
\hline E1 & 1 & 2 & . & + & . & . & . & . & . & . & 3 & 30 \\
\hline E3 & 1 & + & . & . & . & . & . & . & . & . & 2 & 20 \\
\hline E2b & + & + & . & + & . & . & . & . & . & . & 3 & 30 \\
\hline E2a & . & + & . & + & . & . & . & . & . & . & 2 & 20 \\
\hline E1 & . & + & . & . & . & . & . & . & . & . & 1 & 10 \\
\hline E1 & + & + & . & . & . & . & . & . & . & . & 2 & 20 \\
\hline E1 & . & + & . & + & . & . & . & . & . & . & 2 & 20 \\
\hline E1 & . & . & . & + & . & . & . & + & . & . & 2 & 20 \\
\hline E1 & + & . & . & . & . & . & . & . & . & . & 1 & 10 \\
\hline E1 & . & + & . & . & . & . & . & . & . & . & 1 & 10 \\
\hline E1 & . & . & + & . & . & . & . & . & . & . & 1 & 10 \\
\hline E1 & . & . & . & + & . & . & . & . & . & . & 1 & 10 \\
\hline E1 & . & . & . & + & . & . & . & . & . & . & 1 & 10 \\
\hline E2a & . & . & . & + & . & . & . & . & . & . & 1 & 10 \\
\hline E1 & . & . & . & + & . & . & . & . & . & . & 1 & 10 \\
\hline E1 & . & . & . & + & . & . & . & . & . & . & 1 & 10 \\
\hline E1 & . & . & . & 1 & . & . & . & . & . & . & 1 & 10 \\
\hline E1 & . & . & . & . & . & . & + & . & . & . & 1 & 10 \\
\hline
\end{tabular}

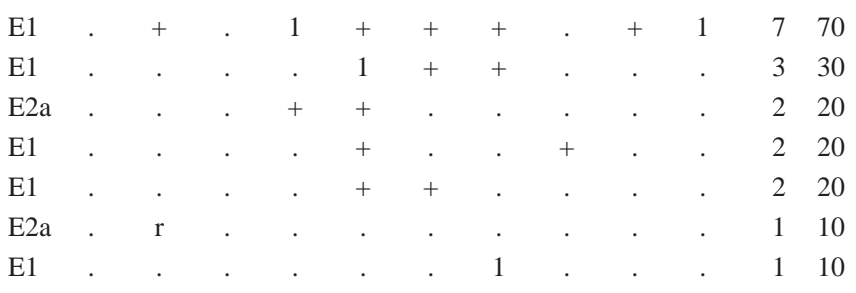

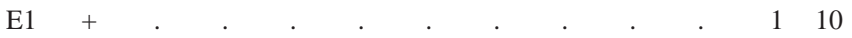

$\mathrm{E} 1 .+. .5 . \quad . \quad . \quad 10$

$\mathrm{E} 2 \mathrm{~b} . \quad . \quad . \quad . \quad . \quad . \quad 10$

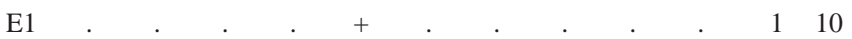

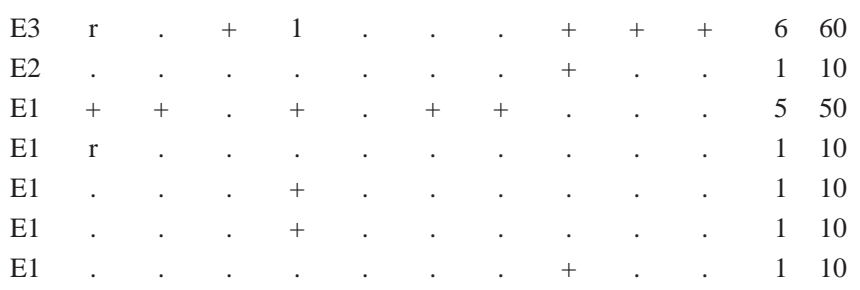

\begin{tabular}{|c|c|c|c|c|c|c|c|c|c|c|c|}
\hline E1 & $\mathrm{r}$ & + & . & 1 & 1 & 1 & + & 1 & 1 & + & 9 \\
\hline E1 & $\mathrm{r}$ & . & . & + & 2 & 2 & 2 & 2 & 2 & 2 & 8 \\
\hline E1 & + & . & . & 1 & 2 & 1 & 1 & 1 & . & . & 6 \\
\hline E1 & $\mathrm{r}$ & . & . & . & 1 & 1 & + & 1 & . & + & 6 \\
\hline E1 & . & . & . & . & 1 & + & + & + & . & . & 4 \\
\hline E1 & . & . & . & . & + & + & + & . & + & . & 4 \\
\hline E3 & + & . & . & . & . & . & . & . & . & . & 1 \\
\hline E2b & . & + & . & . & . & . & . & . & . & . & 1 \\
\hline E2a & + & + & . & . & . & . & . & . & . & . & 2 \\
\hline E1 & + & + & . & . & . & + & . & . & . & . & 3 \\
\hline E3 & $\mathrm{r}$ & . & . & + & $\mathrm{r}$ & . & . & . & . & . & 3 \\
\hline E2a & . & . & . & . & + & . & . & . & . & . & 1 \\
\hline E1 & . & . & . & + & . & . & . & . & . & . & 1 \\
\hline
\end{tabular}


Number of relevé (Zaporedna številka popisa)

Actaea spicata

Lathyrus vernus subsp. vernus

Prenanthes purpurea

Scrophularia nodosa

Daphne mezereum

Phyllitis scolopendrium

Aruncus dioicus

Epilobium montanum

Myosotis sylvatica

Mercurialis perennis

Galeobdolon flavidum

Carex sylvatica

Lonicera alpigena

Ranunculus lanuginosus

Campanula trachelium

Lathyrus vernus subsp. flaccidus

Luzula nivea

QF Querco-Fagetea

Anemone nemorosa

QP Sorbus aria

Scilla bifolia

Dactylorhiza fuchsii

EC Helleborus odorus

ES Elyno-Seslerietea

Sesleria caerulea subsp. calcaria

Festuca calva

Campanula witasekiana

Hieracium pilosum

Carex ferruginea

Heliosperma alpestre

Aster bellidiastrum

Carex sempervirens

FB Koeleria pyramidata

Betonica alopecuros

CU Calluno-Ulicetea

Carex pilulifera

PaT Poo alpinae-Trisetalia

Poa alpina

Trollius europaeus

Pimpinella major subsp. rubra

MA Molinio-Arrhenetheretea

Deschampsia cespitosa

Angelica sylvestris

Taraxacum officinale

TR Thlaspietea rotundifolii

Molopospermum peloponnesiacum subsp. bauhinii

Alchemilla alpigena

Rhodiola rosea

Festuca nitida

Dryopteris villarii

Arabis alpina

Cystopteris montana

Hieracium bifidum

AT Asplenietea trichomanis

Paederota lutea

$\begin{array}{lllllllllllrr} & 1 & 2 & 3 & 4 & 5 & 6 & 7 & 8 & 9 & 10 & \text { Pr. } & \text { Fr. } \\ \text { E1 } & . & . & . & . & + & + & + & . & . & . & 3 & 30 \\ \text { E1 } & . & . & . & . & + & + & + & . & . & . & 3 & 30 \\ \text { E1 } & 2 & 1 & . & . & . & . & . & . & . & . & 2 & 20 \\ \text { E1 } & \text { r } & . & . & + & . & . & . & . & . & . & 2 & 20 \\ \text { E2a } & . & . & . & + & + & . & . & . & . & . & 2 & 20 \\ \text { E1 } & . & . & . & + & . & + & . & . & . & . & 2 & 20 \\ \text { E1 } & . & . & . & . & . & + & . & 1 & . & . & 2 & 20 \\ \text { E1 } & . & . & . & . & . & + & . & 1 & . & . & 2 & 20 \\ \text { E1 } & . & . & + & . & . & . & . & . & . & . & 1 & 10 \\ \text { E1 } & . & . & . & + & . & . & . & . & . & . & 1 & 10 \\ \text { E1 } & . & . & . & . & 1 & . & . & . & . & . & 1 & 10 \\ \text { E1 } & . & . & . & . & + & . & . & . & . & . & 1 & 10 \\ \text { E2a } & . & . & . & . & + & . & . & . & . & . & 1 & 10 \\ \text { E1 } & . & . & . & . & . & + & . & . & . & . & 1 & 10 \\ \text { E1 } & . & . & . & . & . & + & . & . & . & . & 1 & 10 \\ \text { E1 } & . & . & . & . & . & . & + & . & . & . & 1 & 10 \\ \text { E1 } & . & . & . & . & . & . & . & + & . & . & 1 & 10\end{array}$

$\begin{array}{lllllllllllll}\text { E1 } & 1 & 1 & . & . & 1 & 1 & 1 & 1 & + & + & 8 & 80 \\ \text { E2b } & + & + & . & . & + & . & . & . & . & . & 3 & 30 \\ \text { E1 } & \cdot & . & . & . & + & + & . & + & . & . & 3 & 30 \\ \text { E1 } & \cdot & \cdot & . & . & 1 & + & + & . & . & . & 3 & 30 \\ \text { E1 } & . & . & . & . & . & . & . & \text { r } & \text { r } & . & 2 & 20\end{array}$

E1

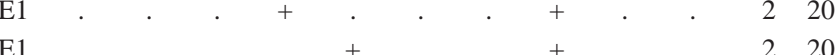

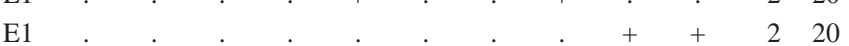

$\mathrm{E} 1 . \quad . \quad . \quad . \quad . \quad . \quad . \quad . \quad 1010$

$\mathrm{E} 1 . .5 . \quad . \quad . \quad . \quad . \quad 110$

$\mathrm{E} 1 . \quad . \quad \mathrm{r} \quad . \quad . \quad . \quad . \quad 10$

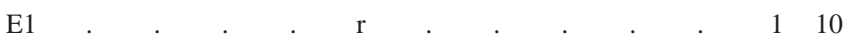

E1 . . . . . . . . . . 1010

$\mathrm{E} 1++. \quad . \quad . \quad . \quad . \quad 20$

$\mathrm{E} 1$. . $.+2+.+330$

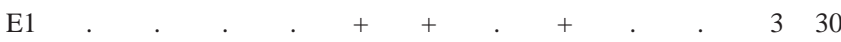

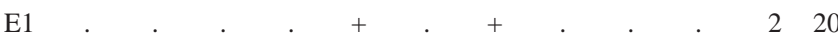

$\begin{array}{lllllllllllll}\mathrm{E} 1 & . & . & 1 & . & 1 & + & + & 1 & + & 2 & 7 & 70 \\ \mathrm{E} 1 & . & . & . & + & 2 & 2 & 1 & 1 & 1 & + & 7 & 70 \\ \mathrm{E} 1 & . & . & . & . & . & . & . & \mathrm{r} & . & . & 1 & 10\end{array}$

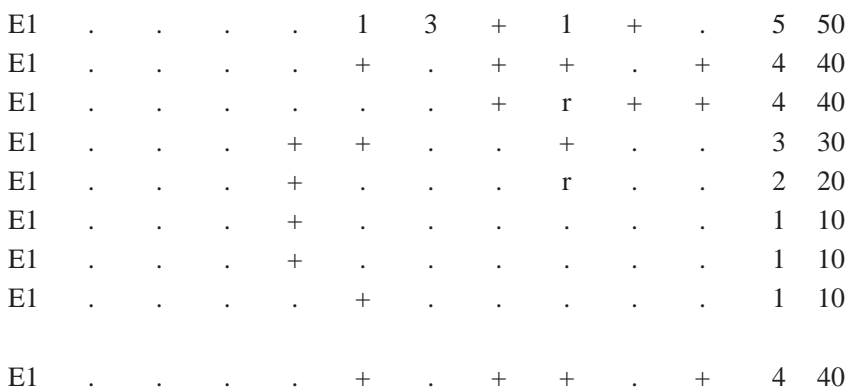


Number of relevé (Zaporedna številka popisa)

Campanula carnica

Asplenium trichomanes

Sedum maximum

Festuca stenantha

Primula auricula

Asplenium ruta-muraria

Cerastium subtriflorum

Saxifraga crustata

O Other species (Druge vrste)

Alchemilla sp.

ML Mosses and lichens (Mahovi in lišaji)

Rhytidiadelphus triquetrus

Ctenidium molluscum

Polytrichum formosum

Tortella tortuosa

Isothecium alopecuroides

Schistidium apocarpum

Mnium thomsonii

Eurhynchium striatum

Pseudoleskeella catenulata

Hylocomium splendens

Rhytidiadelphus loreus

Cladonia pyxidata

Dicranum scoparium

Marchantia polymorpha

Peltigera canina

Atrichum undulatum

\begin{tabular}{|c|c|c|c|c|c|c|c|c|c|c|c|c|}
\hline & 1 & 2 & 3 & 4 & 5 & 6 & 7 & 8 & 9 & 10 & Pr. & Fr. \\
\hline E1 & . & . & . & . & + & + & . & . & . & . & 2 & 20 \\
\hline E1 & . & . & . & . & + & + & . & . & . & . & 2 & 20 \\
\hline E1 & . & . & . & + & . & . & . & . & . & . & 1 & 10 \\
\hline E1 & . & . & . & . & + & . & . & . & . & . & 1 & 10 \\
\hline E1 & . & . & . & . & + & . & . & . & . & . & 1 & 10 \\
\hline E1 & . & . & . & . & + & . & . & . & . & . & 1 & 10 \\
\hline E1 & . & . & . & . & . & . & + & . & . & . & 1 & 10 \\
\hline E1 & . & . & . & . & . & . & . & $\mathrm{r}$ & . & . & 1 & 10 \\
\hline
\end{tabular}

A Limestone - apnenec

G Claystone - glinavec

R Chert-roženec

L Marl-laporovec

Re Rendzina - rendzina

Dy Dystric brown soil - distrična rjava tla

K Brown calcareous soil - rjava pokarbonatna tla

ID Igor Dakskobler

Maximum tree diametre: $15-30 \mathrm{~cm}$

Maximum tree height: 6-12 m 
Table 7 / Tabela 7: Alno viridis-Aceretum pseudoplatani nom. prov.

Number of relevé (Zaporedna številka popisa)

1

Database number of relevé (Delovna številka popisa)

220753

Elevation in $\mathrm{m}$ (Nadmorska višina v m)

1430

Aspect (Lega)

W

Slope in degrees (Nagib v stopinjah)

30

Parent material (Matična podlaga)

DA

Soil (Tla)

Re

Stoniness in \% (Kamnitost v \%)

Cover of tree layer in \% (Zastiranje drevesne plasti v \%)

20

Cover of shrub layer in \% (Zastiranje grmovne plasti v \%):

60

Cover of herb layer in \% (Zastiranje zeliščne plasti v \%):

60

Cover of moss layer in \% (Zastiranje mahovne plasti v \%)

E2

E1

E0

90

Number of species (Število vrst)

Relevé area (Velikost popisne ploskve)

$\mathrm{m}^{2} \quad 400$

Date of taking relevé (Datum popisa)

9/10/2008

Locality (Nahajališče)

Komar

Quadrant (Kvadrant)

9648/2

Coordinate GK Y (D-48)

408799

Coordinate GK X (D-48)

$\mathrm{m}$

137226

Author of the relevé (Avtor popisa)

TA Tilio-Acerion

Acer pseudoplatanus

E3b

Acer pseudoplatanus

E2b

Acer pseudoplatanus

E2a

Acer pseudoplatanus

E1

Thalictrum aquilegiifolium

E1

BA Betulo carpaticae-Alnetea viridis

Alnus viridis

E2b

Salix appendiculata

Salix appendiculata

MuA Mulgedio-Aconitetea

Aconitum lycoctonum subsp. ranunculifolium

Polygonatum verticillatum

Senecio cacaliaster

Athyrium filix-femina

Chaerophyllum villarsii

Aconitum degenii subsp. paniculatum

$\begin{array}{ll}\text { E1 } & 1 \\ \text { E1 } & 1 \\ \text { E1 } & 1 \\ \text { E1 } & + \\ \text { E1 } & + \\ \text { E1 } & \text { r }\end{array}$

AF Aremonio-Fagion

Cyclamen purpurascens

Anemone trifolia

Fagetalia sylvaticae

Actaea spicata

Daphne mezereum

Galium laevigatum

Lonicera alpigena

Melica nutans

Mercurialis perennis

Phyteuma spicatum

Prenanthes purpurea

Laburnum alpinum

Laburnum alpinum

Paris quadrifolia

E1

Dryopteris filix-mas

E1

Galeobdolon flavidum 
Number of relevé (Zaporedna številka popisa)

1

\begin{tabular}{|c|c|c|c|}
\hline \multirow[t]{2}{*}{$\overline{\mathrm{QP}}$} & \multicolumn{3}{|l|}{ Quercetalia pubescentis } \\
\hline & Sorbus aria & E3b & $\mathrm{r}$ \\
\hline \multirow[t]{9}{*}{ EP } & Erico-Pinetea & & \\
\hline & Calamagrostis varia & E1 & 3 \\
\hline & Erica carnea & E1 & 1 \\
\hline & Aquilegia nigricans & E1 & 1 \\
\hline & Pinus mugo & $\mathrm{E} 2 \mathrm{~b}$ & 1 \\
\hline & Rubus saxatilis & E1 & 1 \\
\hline & Buphthalmum salicifolium & E1 & $\mathrm{r}$ \\
\hline & Carex ornithopoda & E1 & + \\
\hline & Peucedanum austriacum subsp. rablense & E1 & + \\
\hline \multirow[t]{18}{*}{ VP } & Vaccinio-Piceetea & & \\
\hline & Lycopodium annotinum & E1 & 1 \\
\hline & Polystichum lonchitis & E1 & 1 \\
\hline & Rosa pendulina & E2a & 1 \\
\hline & Aposeris foetida & E1 & 1 \\
\hline & Clematis alpina & E2a & + \\
\hline & Larix decidua & $\mathrm{E} 2 \mathrm{~b}$ & + \\
\hline & Larix decidua & E2a & $\mathrm{r}$ \\
\hline & Lonicera nigra & E2a & + \\
\hline & Picea abies & E3b & + \\
\hline & Picea abies & $\mathrm{E} 2 \mathrm{~b}$ & + \\
\hline & Picea abies & E2a & $\mathrm{r}$ \\
\hline & Vaccinium myrtillus & E1 & + \\
\hline & Valeriana tripteris & E1 & + \\
\hline & Dryopteris dilatata & E1 & + \\
\hline & Gymnocarpium dryopteris & E1 & + \\
\hline & Huperzia selago & E1 & + \\
\hline & Abies alba & $\mathrm{E} 2 \mathrm{~b}$ & $\mathrm{r}$ \\
\hline \multirow[t]{3}{*}{ SSC } & Sambuco-Salicion capreae & & \\
\hline & Sorbus aucuparia & $\mathrm{E} 2 \mathrm{~b}$ & + \\
\hline & Sorbus aucuparia & E1 & + \\
\hline \multirow[t]{4}{*}{ ES } & Elyno-Seslerietea & & \\
\hline & Betonica alopecuros & E1 & + \\
\hline & Scabiosa lucida subsp. stricta & E1 & + \\
\hline & Arabis ciliata & E1 & + \\
\hline \multirow[t]{2}{*}{ FB } & Festuco-Brometea & & \\
\hline & Gentianella ciliata & E1 & $\mathrm{r}$ \\
\hline \multirow[t]{3}{*}{ AT } & Asplenietea trichomanis & & \\
\hline & Moehringia muscosa & E1 & + \\
\hline & Asplenium viride & E1 & + \\
\hline \multirow[t]{3}{*}{ TR } & Thlaspietea rotundifolii & & \\
\hline & Gymnocarpium robertianum & E1 & + \\
\hline & Valeriana montana & E1 & + \\
\hline \multirow[t]{6}{*}{ ML } & Mosses (Mahovi) & & \\
\hline & Ctenidium molluscum & E0 & 1 \\
\hline & Polytrichum formosum & E0 & + \\
\hline & Rhytidiadelphus triquetrus & E0 & + \\
\hline & Tortella tortuosa & E0 & + \\
\hline & Rhodobryum roseum ? & E0 & + \\
\hline
\end{tabular}

A Limestone - apneenc

D Dolomite - dolomit

Re Rendzina - rendzina

ID Igor Dakskobler 


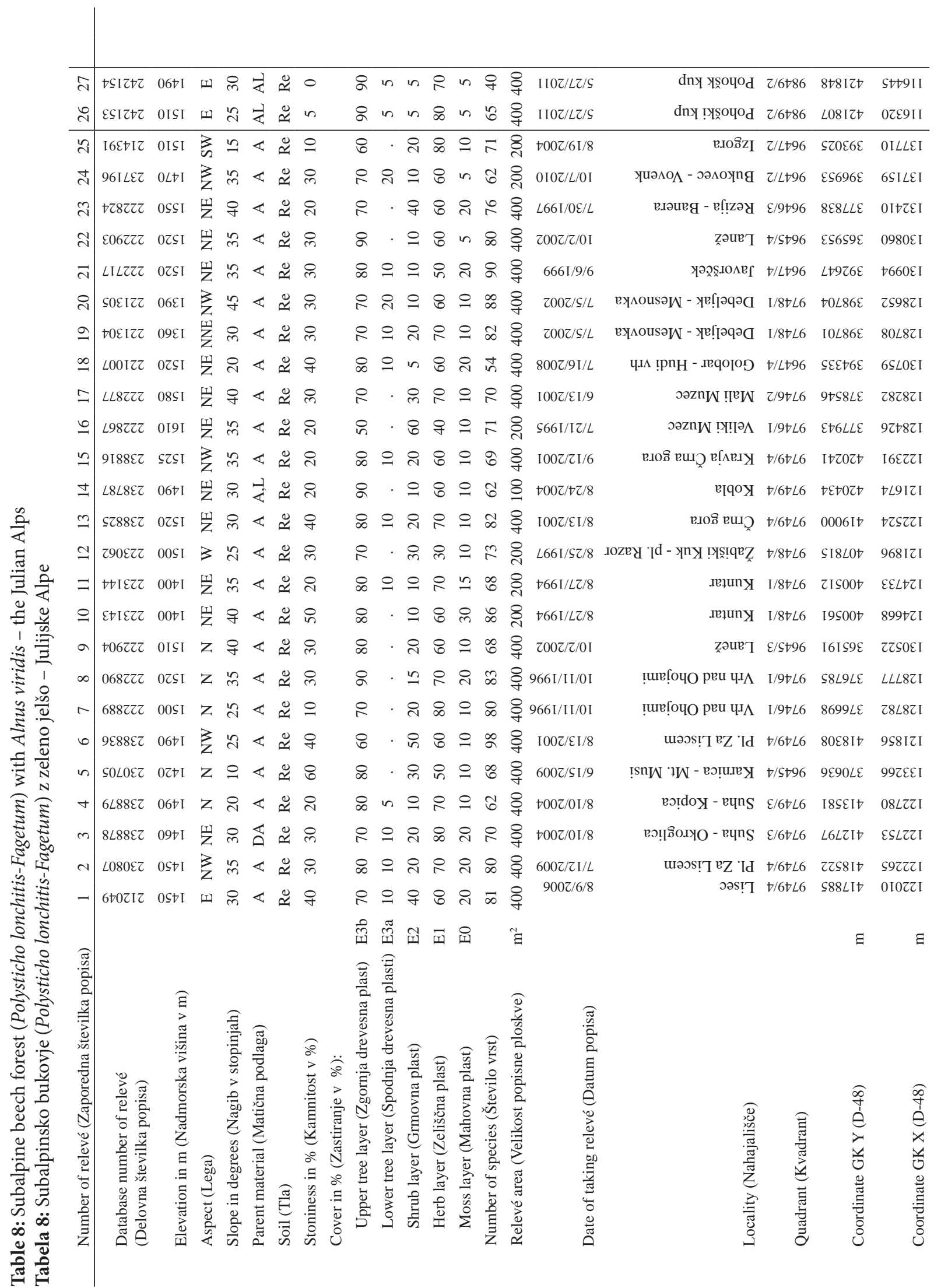


庄号品 萠

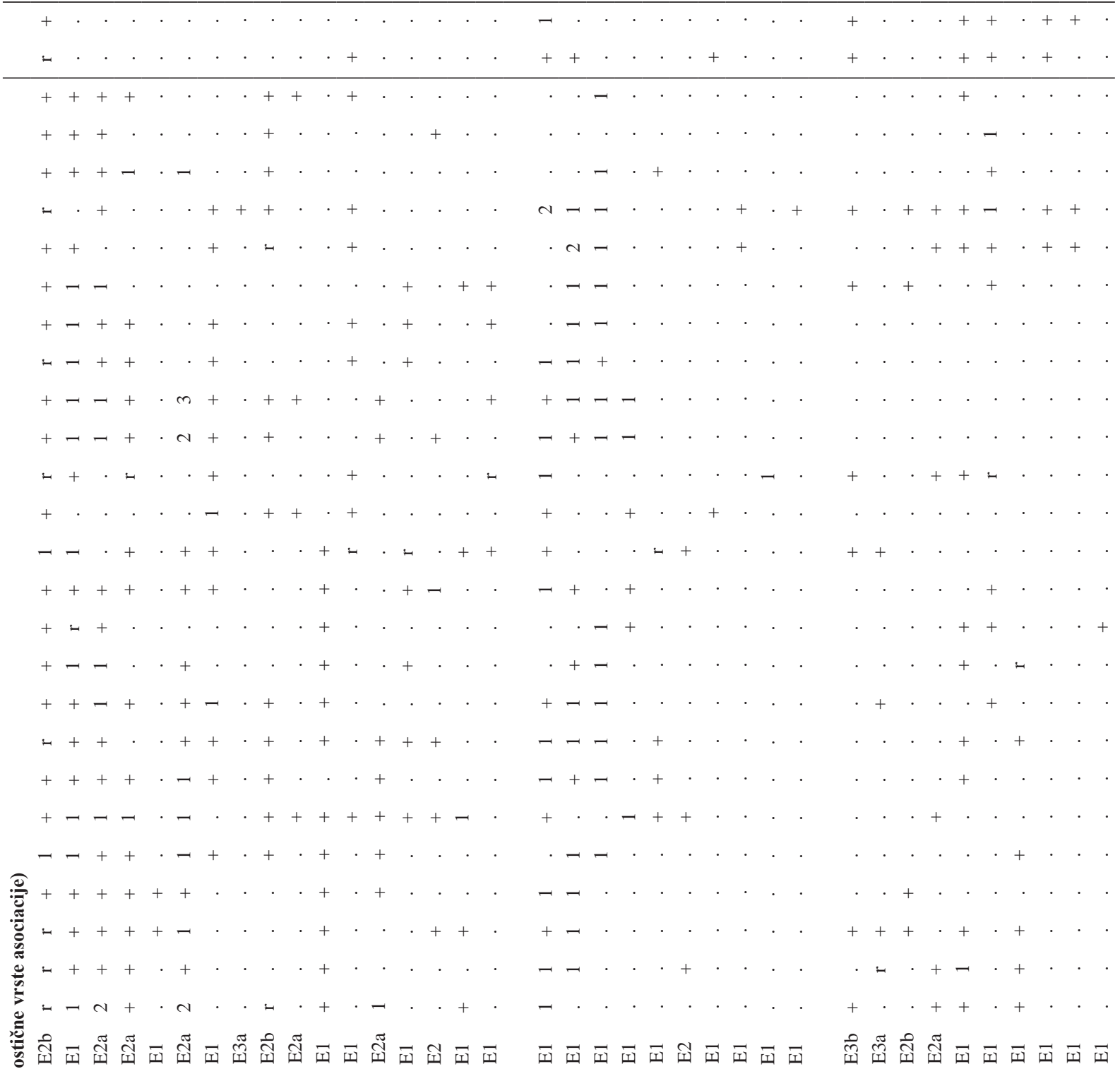




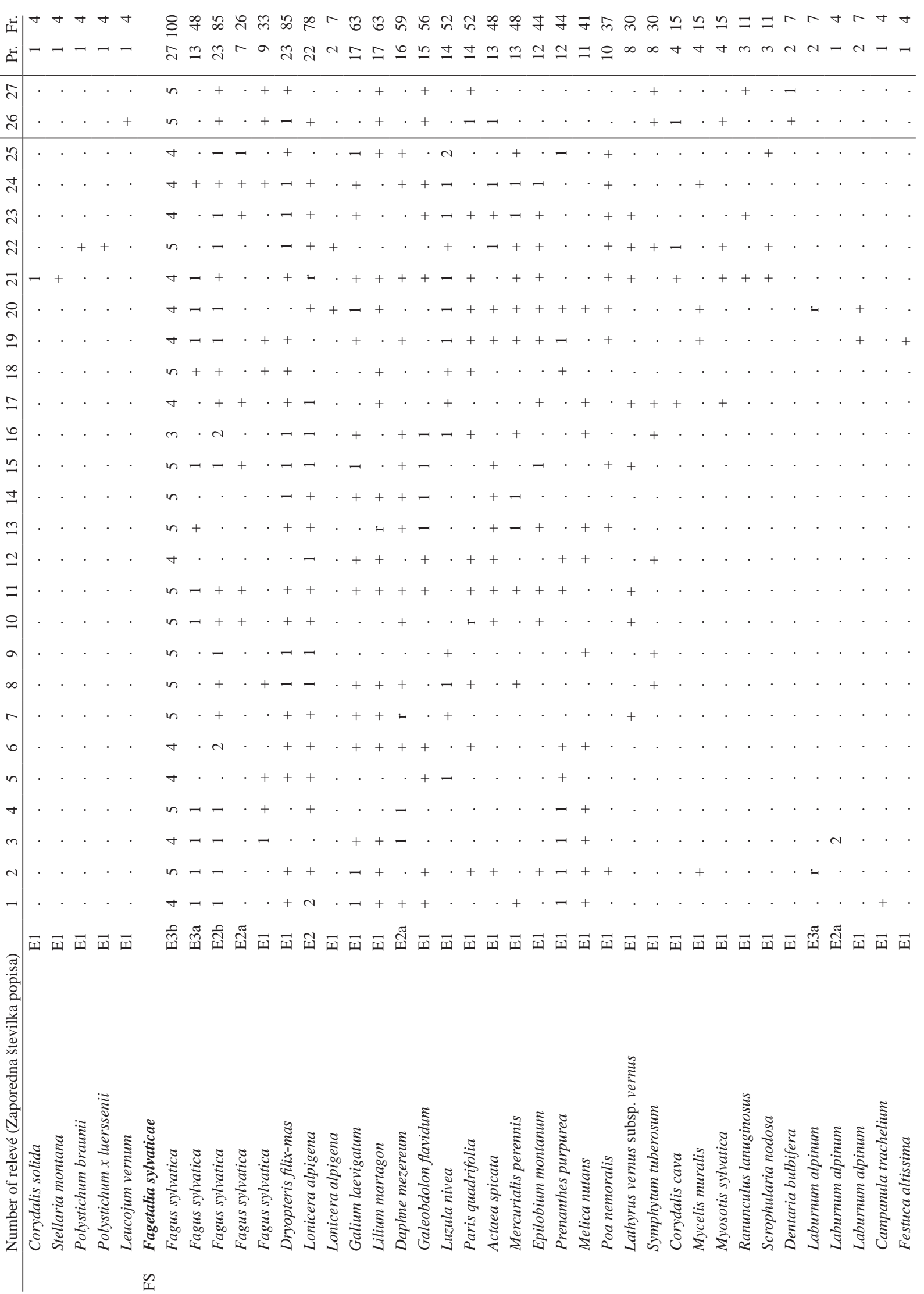




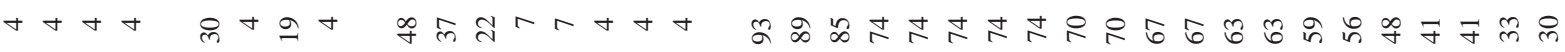

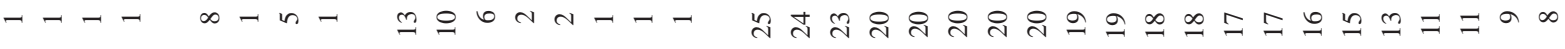

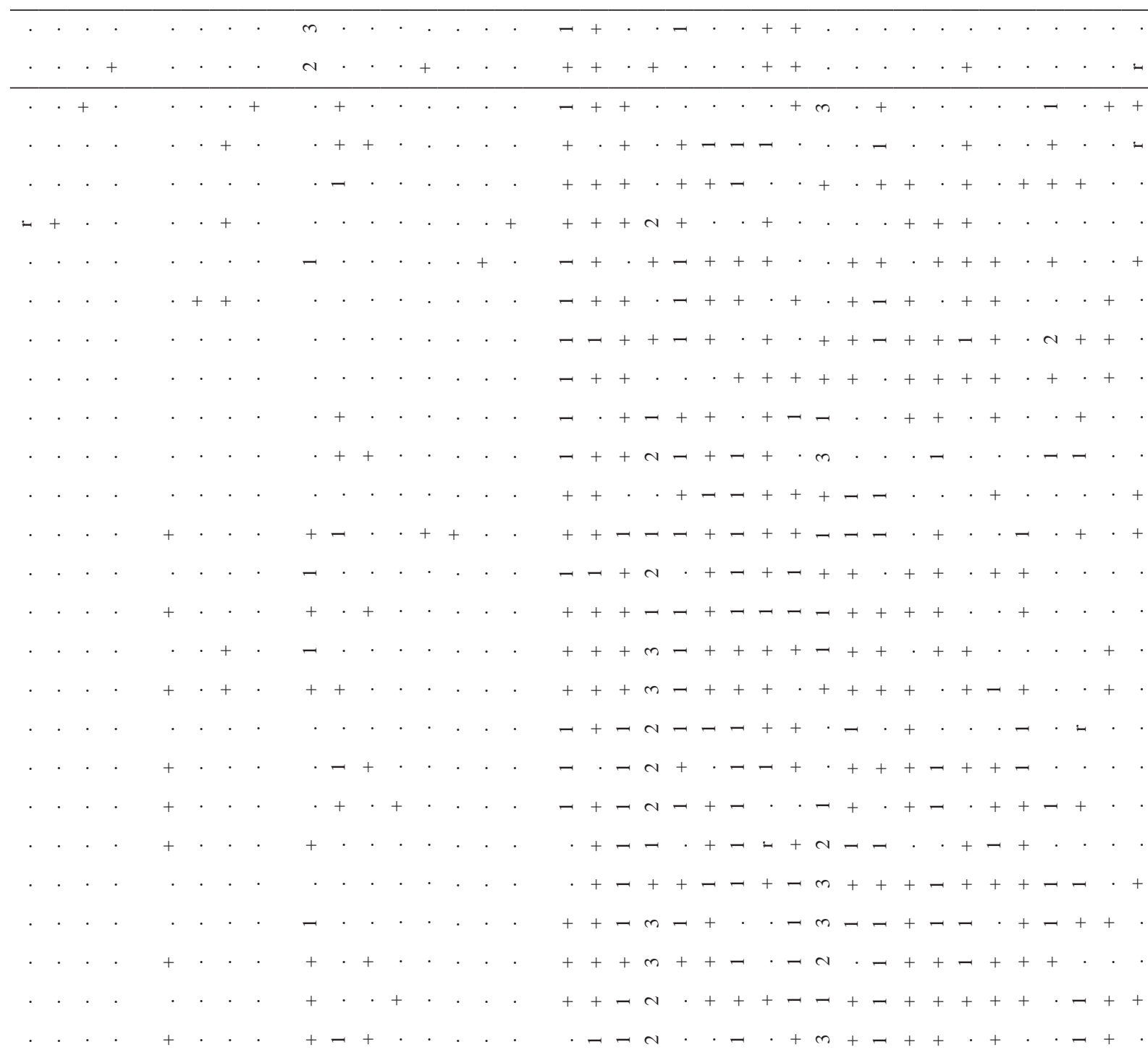

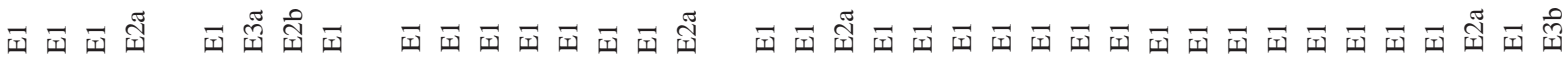

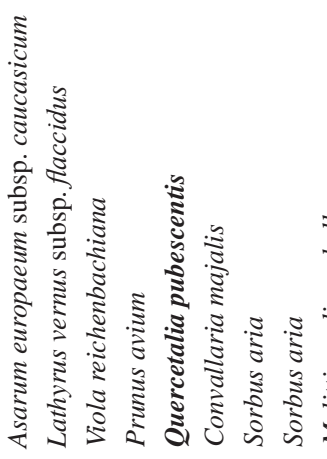

ซ
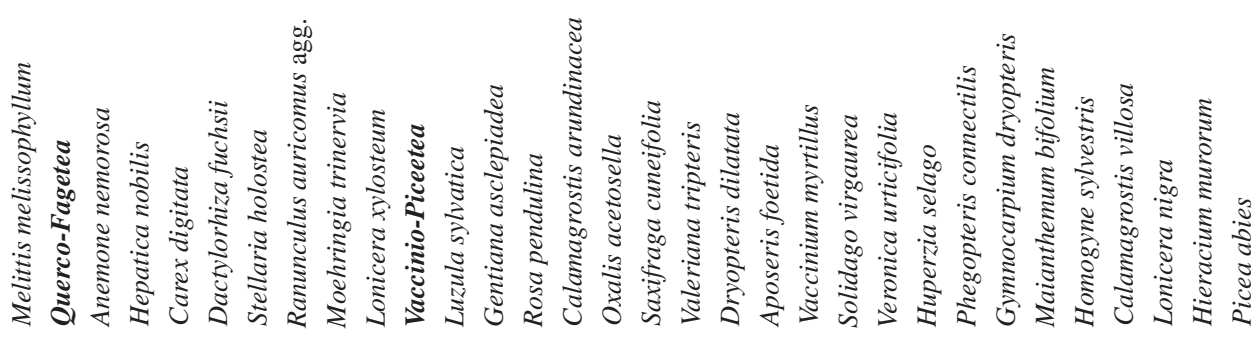

땅

s 


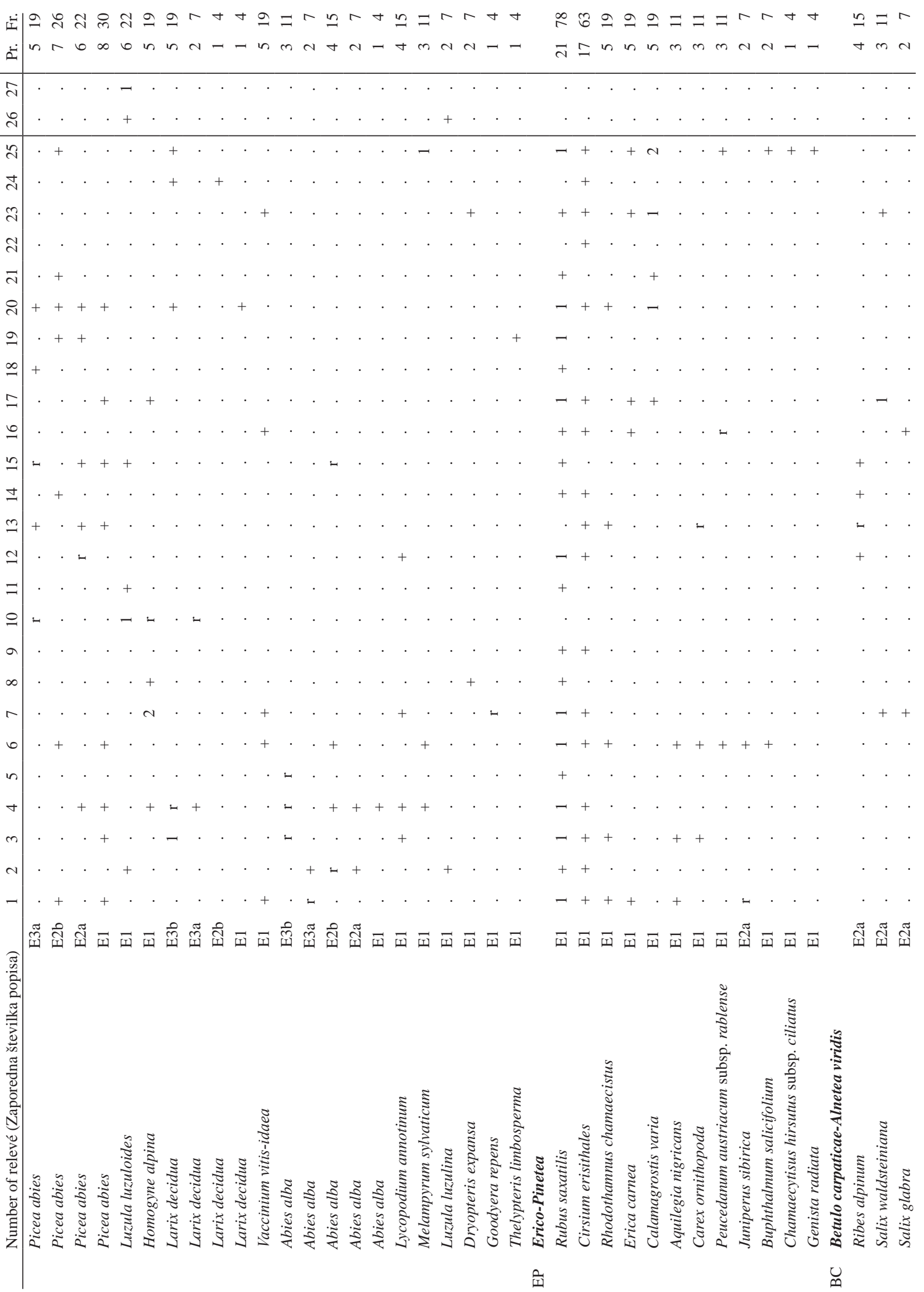




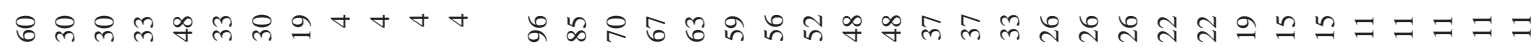

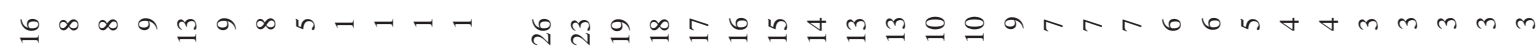

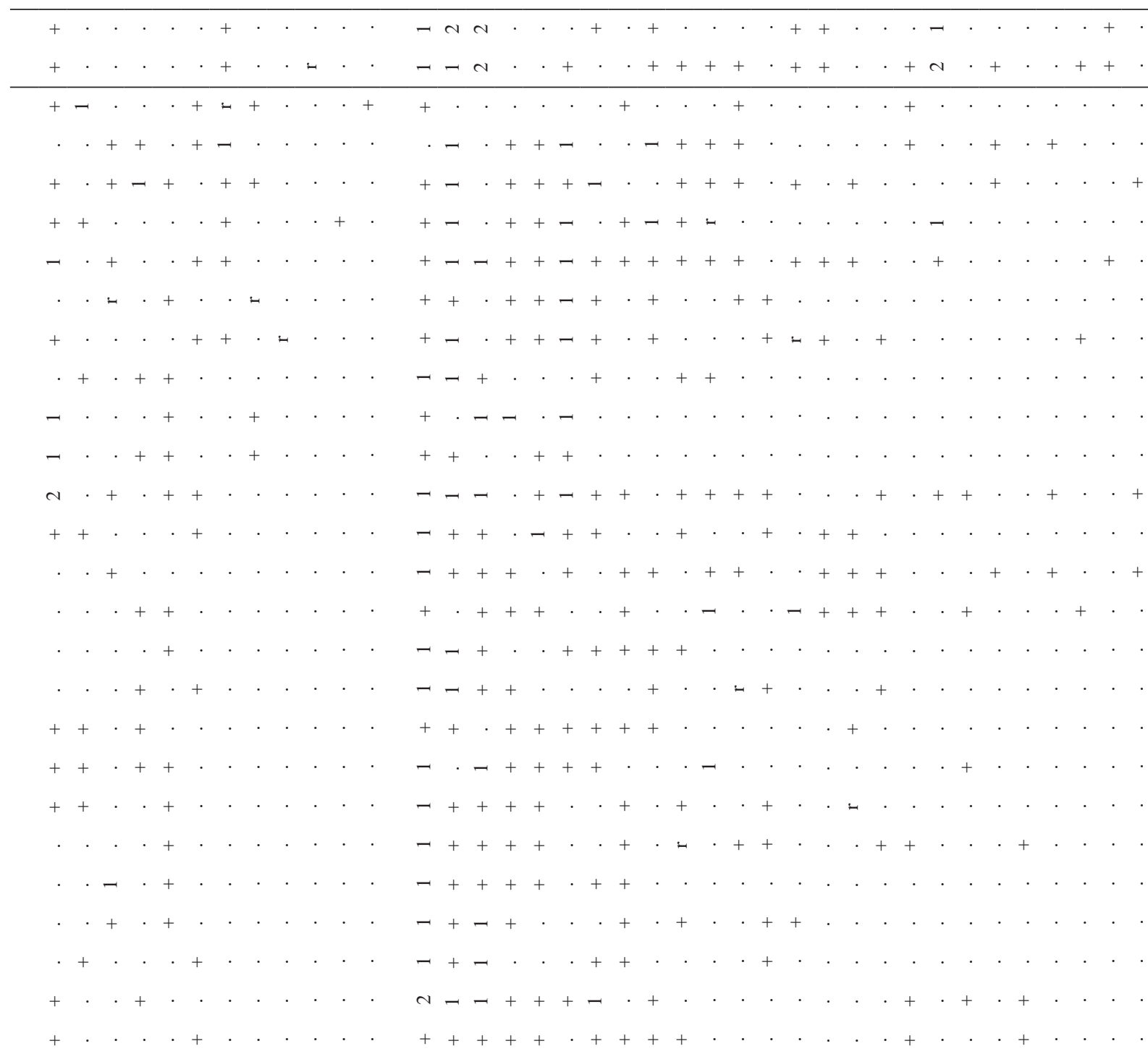

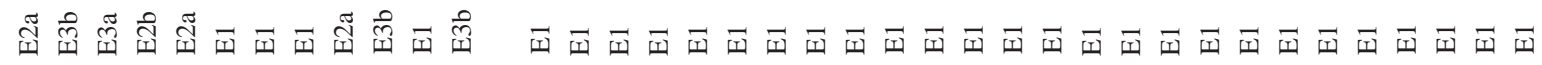

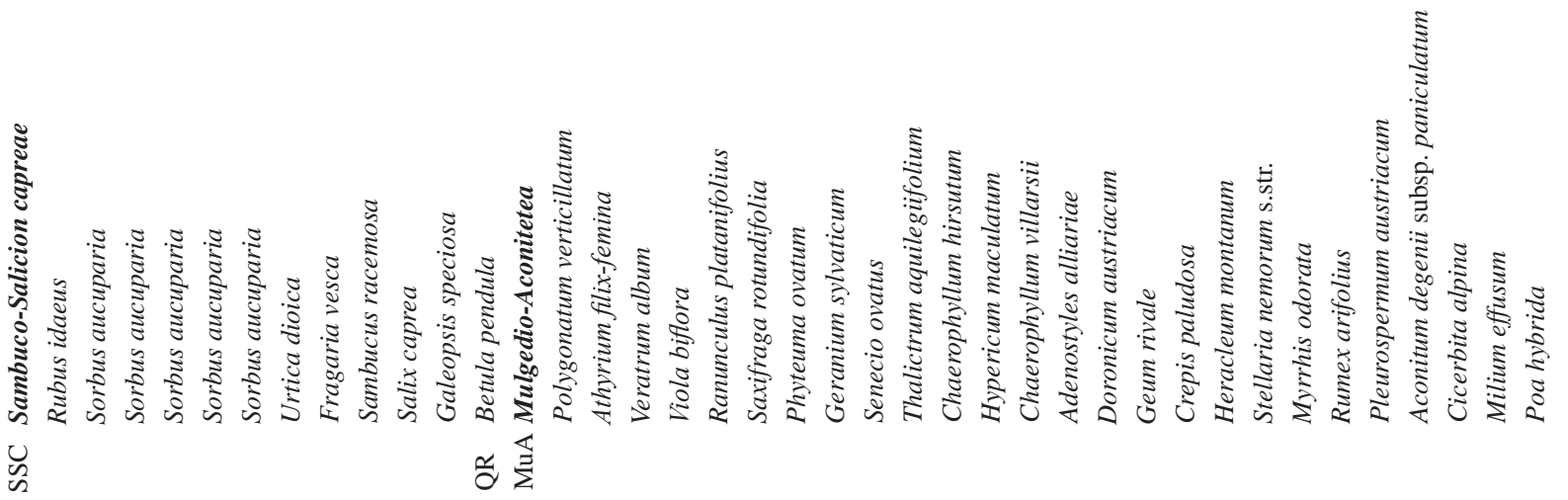




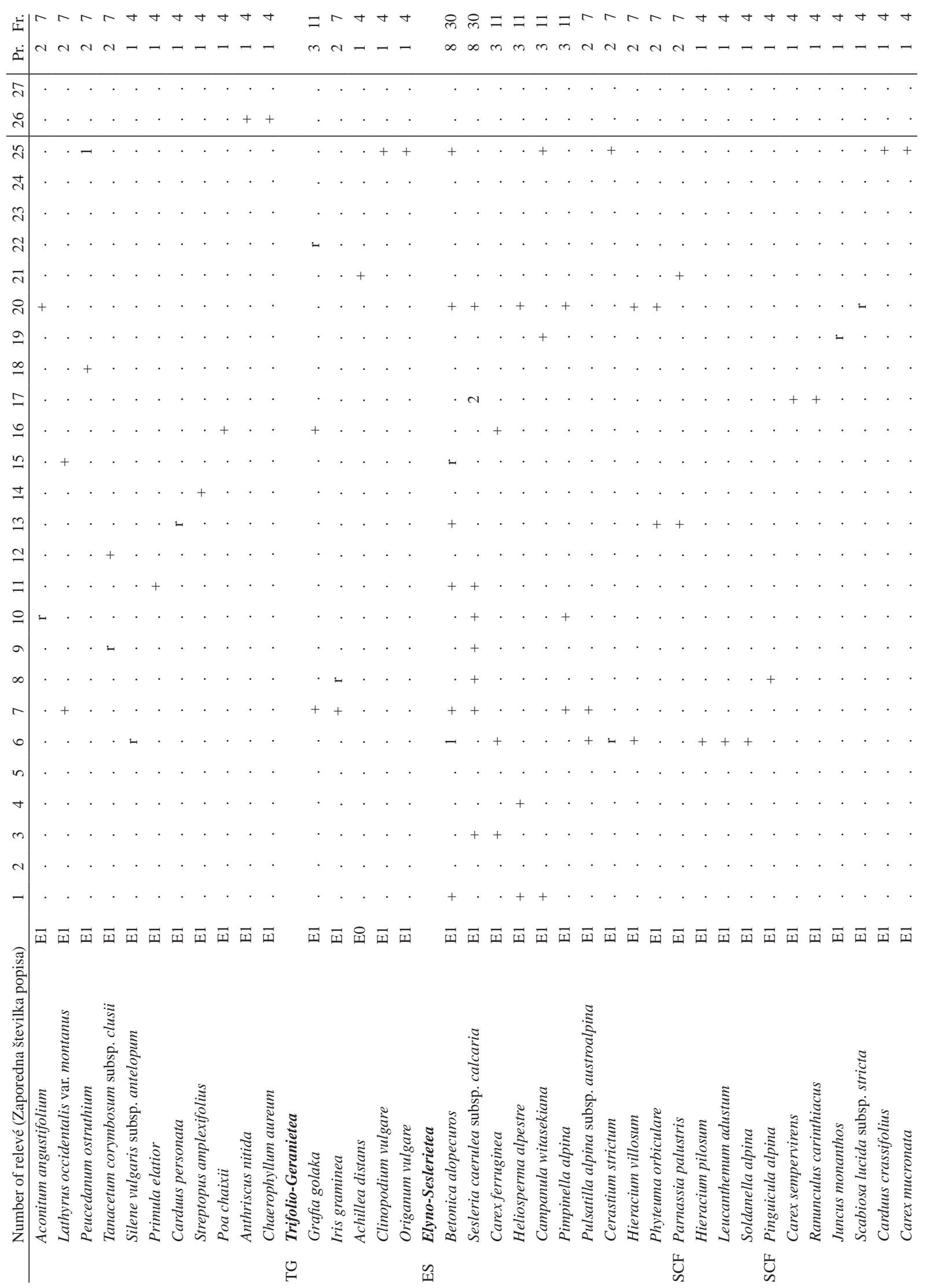




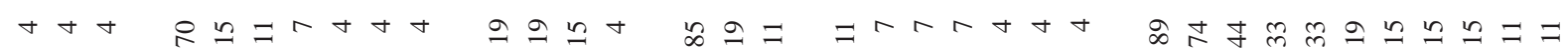

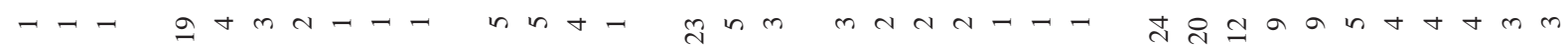

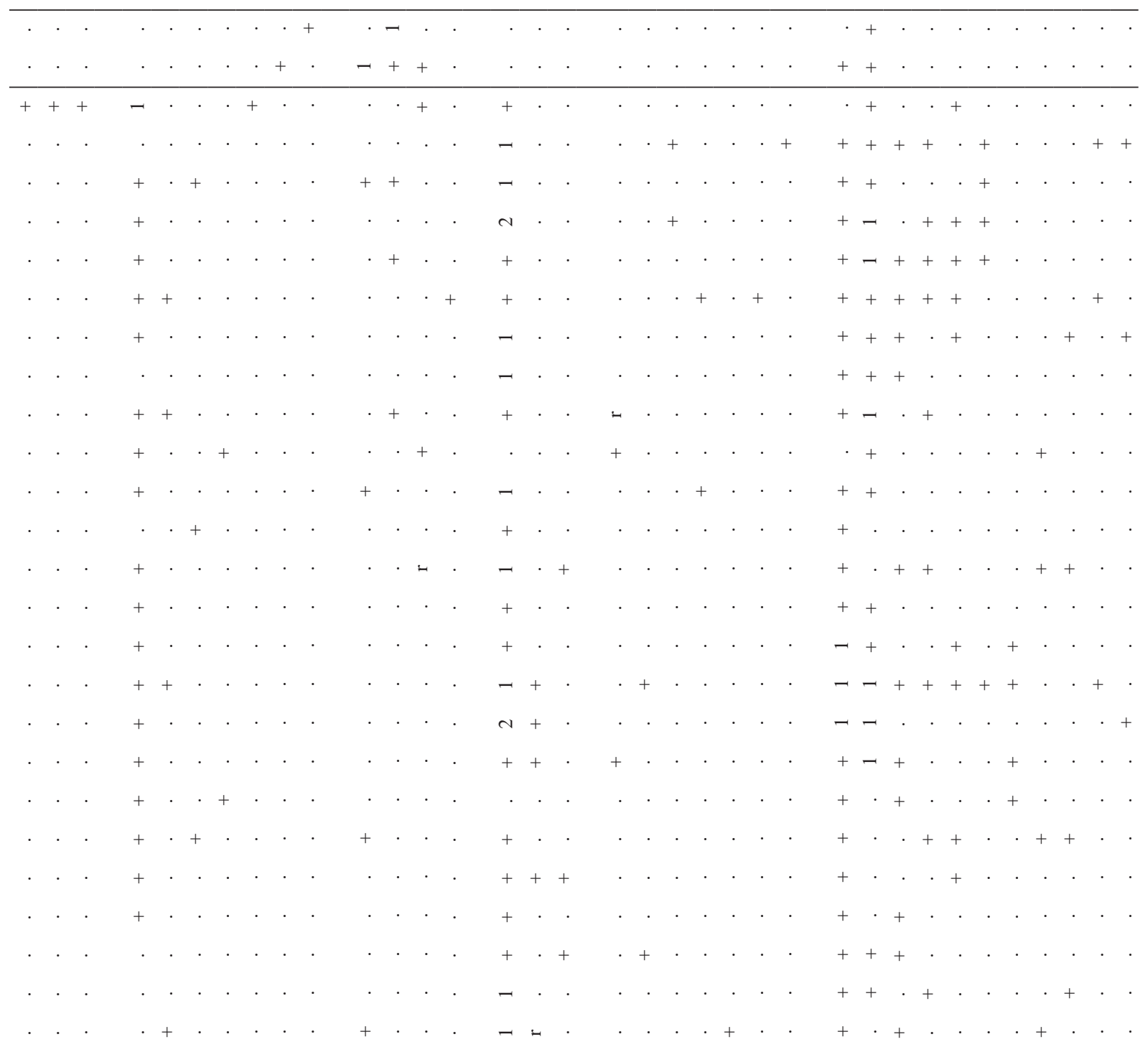

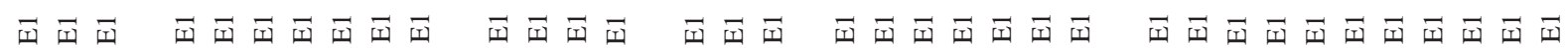

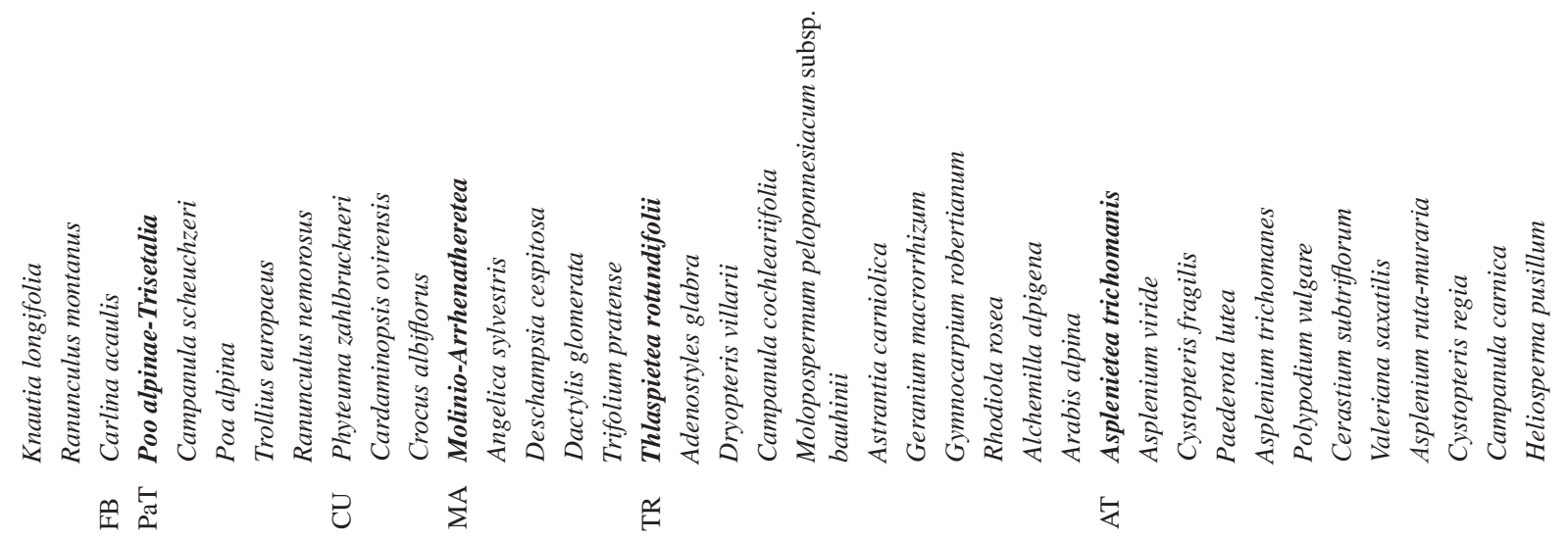




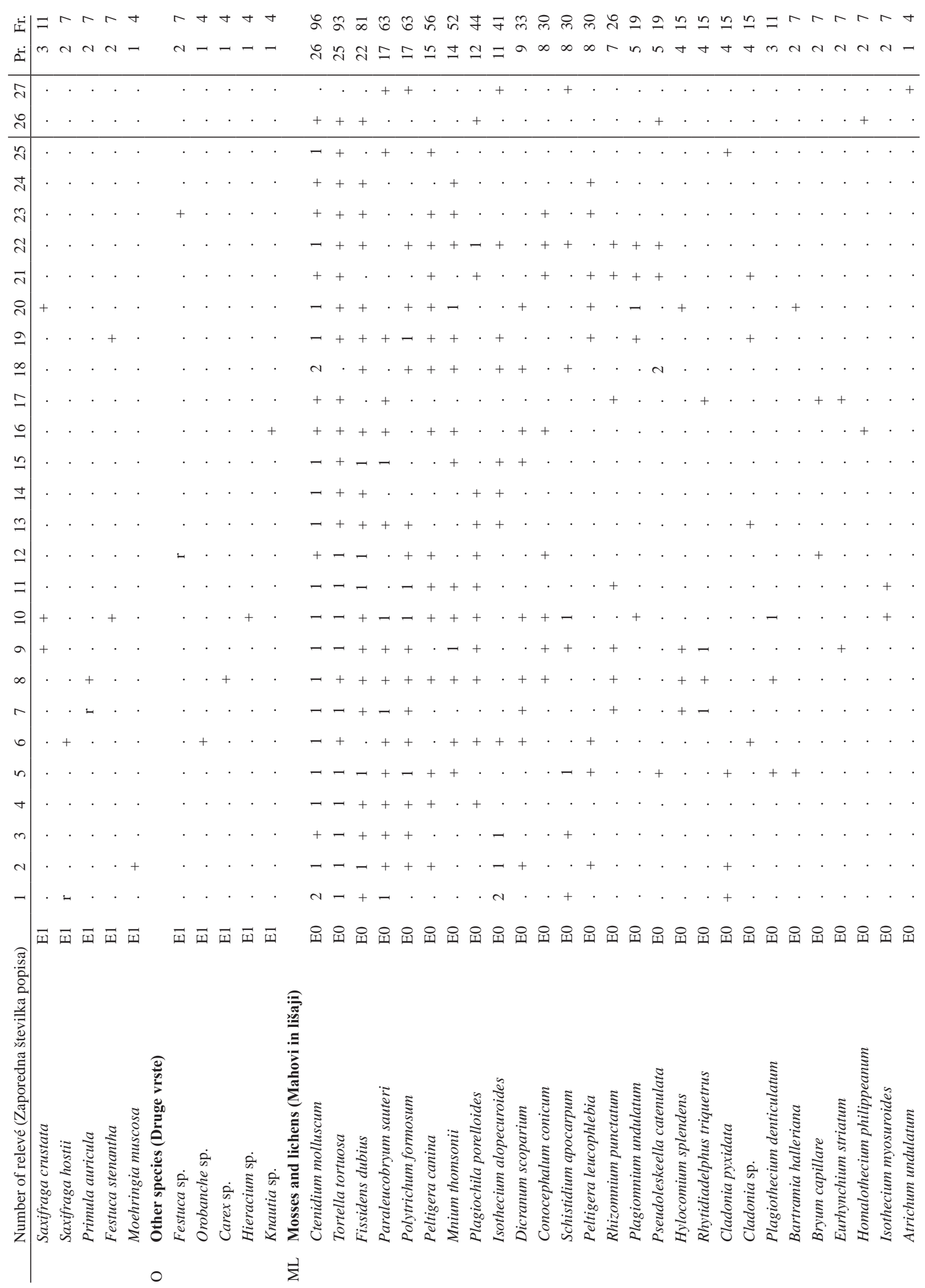




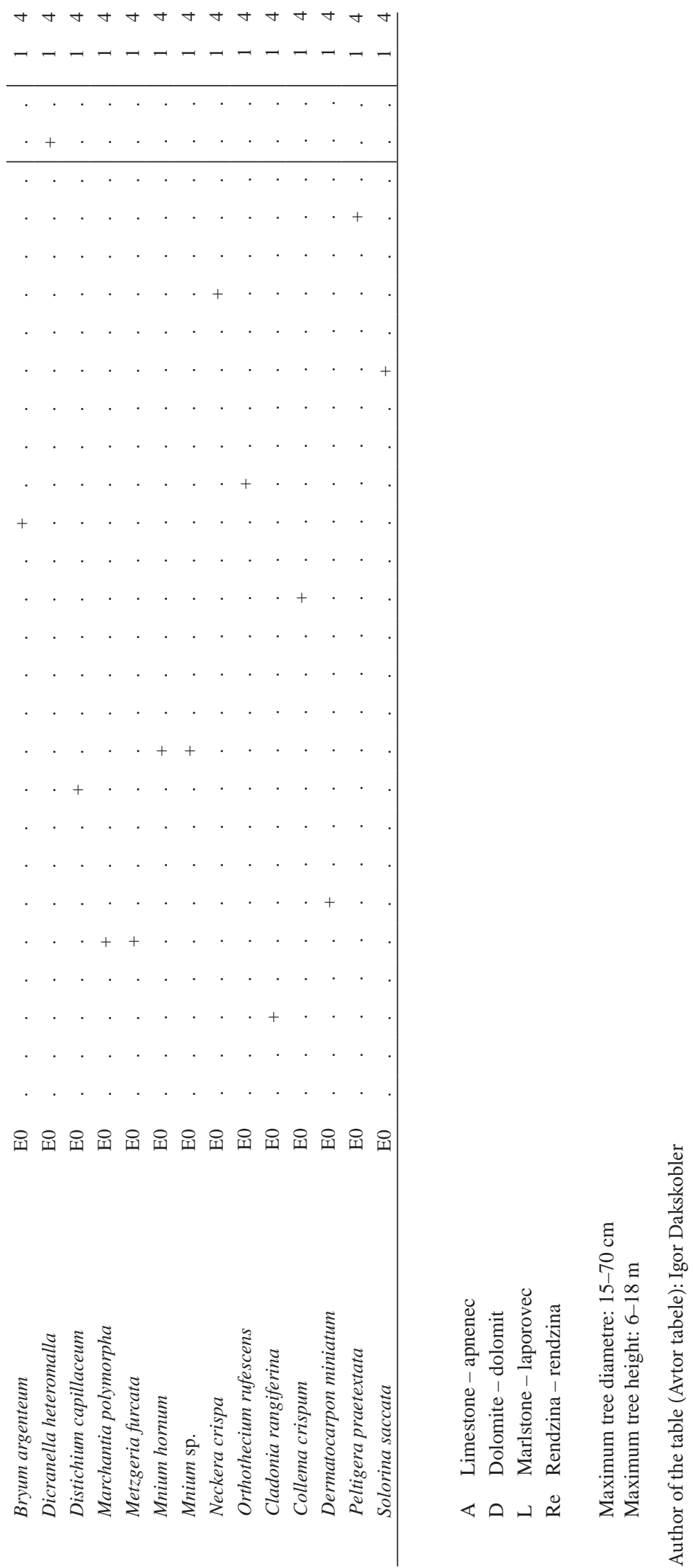


Table 9: Subalpine larch stands (Rhodothamno-Laricetum) with Alnus viridis

\begin{tabular}{|c|c|c|c|c|c|c|c|c|c|c|c|c|c|c|c|c|}
\hline Number of relevé (Zaporedna številka popisa) & & 1 & 2 & 3 & 4 & 5 & 6 & 7 & 8 & 9 & 10 & 11 & 12 & 13 & 14 & 15 \\
\hline $\begin{array}{l}\text { Database number of relevé } \\
\text { (Delovna številka popisa) }\end{array}$ & & 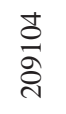 & 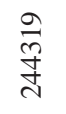 & 岕 & $\begin{array}{l}\vec{b} \\
\stackrel{y}{J} \\
\stackrel{J}{*}\end{array}$ & $\begin{array}{l}\stackrel{v}{~} \\
\text { के }\end{array}$ & 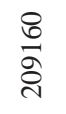 & $\begin{array}{l}\text { गे } \\
\text { Oे } \\
\text { }\end{array}$ & $\begin{array}{l}\text { Dे } \\
\text { ڤે } \\
\text { ठ }\end{array}$ & 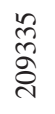 & 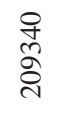 & $\begin{array}{l}\text { సे } \\
\text { के } \\
\text { }\end{array}$ & $\begin{array}{l}\vec{m} \\
\ddot{\circledast} \\
\stackrel{\sim}{2}\end{array}$ & $\begin{array}{l}\text { ₹ै } \\
\text { गे } \\
\text { ᄋे }\end{array}$ & 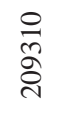 & $\begin{array}{l}\text { ָิ } \\
\text { จ }\end{array}$ \\
\hline Elevation in m (Nadmorska višina v m) & & 衤 & $\begin{array}{l}\stackrel{8}{0} \\
\stackrel{1}{-1}\end{array}$ & 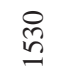 & 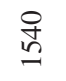 & 昂 & 官 & $\stackrel{8}{8}$ & $\stackrel{\nwarrow}{\emptyset}$ & 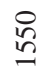 & 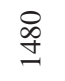 & 命 & 号 & $\begin{array}{l}\stackrel{8}{6} \\
\stackrel{1}{-1}\end{array}$ & 옹 & 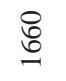 \\
\hline Aspect (Lega) & & $\mathrm{N}$ & $\mathrm{N}$ & $\mathrm{N}$ & $\mathrm{NE}$ & $\mathrm{NE}$ & $\mathrm{N}$ & $\mathrm{N}$ & NW & $\mathrm{N}$ & $\mathrm{NE}$ & $\mathrm{NE}$ & NE & $\mathrm{N}$ & $\mathrm{N}$ & NE \\
\hline Slope in degrees (Nagib v stopinjah) & & 45 & 40 & 50 & 50 & 35 & 40 & 45 & 35 & 50 & 40 & 45 & 35 & 45 & 40 & 35 \\
\hline Parent material (Matična podlaga) & & A & A & A & DA & DA & A & A & A & A & A & A & A & A & A & $\mathrm{D}$ \\
\hline Soil (Tla) & & $\operatorname{Re}$ & $\operatorname{Re}$ & Re & $\mathrm{Re}$ & $\operatorname{Re}$ & $\mathrm{Re}$ & $\operatorname{Re}$ & $\mathrm{Re}$ & $\operatorname{Re}$ & $\operatorname{Re}$ & $\operatorname{Re}$ & Re & $\mathrm{Re}$ & $\operatorname{Re}$ & $\operatorname{Re}$ \\
\hline Stoniness in \% (Kamnitost v \%) & & 40 & 20 & 30 & 40 & 10 & 40 & 20 & 30 & 60 & 50 & 50 & 80 & 40 & 20 & 10 \\
\hline \multicolumn{17}{|l|}{ Cover in \% (Zastiranje v \%): } \\
\hline Upper tree layer (Zgornja drevesna plast) & E3b & 60 & 60 & 50 & 50 & 60 & 60 & 60 & 70 & 60 & 60 & 60 & 60 & 50 & 20 & 30 \\
\hline Lower tree layer (Spodnja drevesna plasti) & E3a & 10 & 20 & & 10 & & 20 & 5 & 10 & 10 & 20 & 10 & 10 & & & \\
\hline Shrub layer (Grmovna plast) & E2 & 30 & 30 & 20 & 20 & 60 & 20 & 40 & 30 & 40 & 50 & 30 & 50 & 50 & 80 & 90 \\
\hline Herb layer (Zeliščna plast) & E1 & 70 & 80 & 70 & 70 & 60 & 60 & 80 & 60 & 60 & 60 & 60 & 60 & 30 & 40 & 70 \\
\hline Moss layer (Mahovna plast) & E0 & 10 & 20 & 10 & 10 & 10 & 10 & 20 & 10 & 20 & 30 & 20 & 20 & 60 & 20 & 20 \\
\hline Number of species (Število vrst) & & 92 & 70 & 65 & 74 & 94 & 73 & 94 & 89 & 94 & 80 & 122 & 91 & 74 & 58 & 50 \\
\hline Relevé area (Velikost popisne ploskve) & $\mathrm{m}^{2}$ & 200 & 400 & 400 & 400 & 400 & 200 & 400 & 400 & 400 & 200 & 400 & 200 & 200 & 200 & 200 \\
\hline Date of taking relevé (Datum popisa) & & 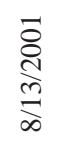 & $\begin{array}{l}\stackrel{N}{\sigma} \\
\stackrel{N}{N} \\
\stackrel{\infty}{\infty}\end{array}$ & 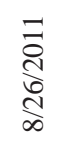 & 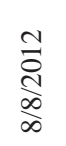 & 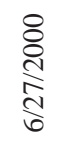 & 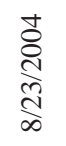 & 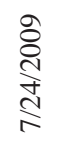 & 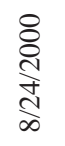 & 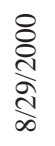 & 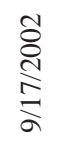 & $\frac{\stackrel{9}{\sigma}}{\stackrel{\rho}{\infty}}$ & $\begin{array}{l}\stackrel{-}{\circ} \\
\stackrel{1}{\omega} \\
\stackrel{1}{\sigma}\end{array}$ & 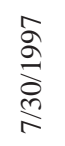 & $\frac{8}{\stackrel{ }{ }}$ & $\underset{\stackrel{N}{\stackrel{N}{N}}}{\sigma}$ \\
\hline Locality (Nahajališče) & & 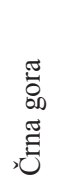 & 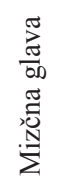 & 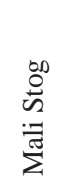 & 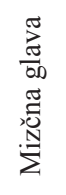 & 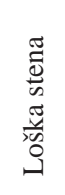 & 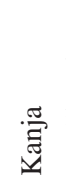 & 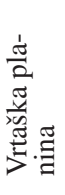 & 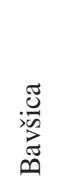 & 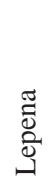 & 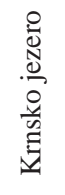 & 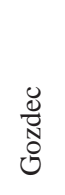 & 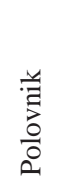 & : & 范 & 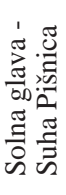 \\
\hline Quadrant (Kvadrant) & & 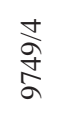 & $\begin{array}{l}\stackrel{+}{\infty} \\
\stackrel{+}{+}\end{array}$ & $\begin{array}{l}\frac{m}{a} \\
\text { ఫै }\end{array}$ & 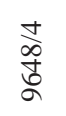 & 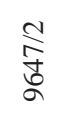 & 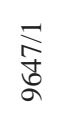 & 守 & 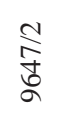 & 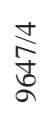 & 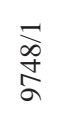 & 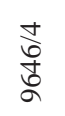 & 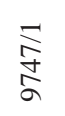 & 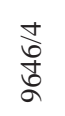 & $\frac{\stackrel{N}{~}}{\mathscr{\sigma}}$ & $\begin{array}{l}\stackrel{+}{\infty} \\
\text { ঢ̛ } \\
\text { ঢ̆ }\end{array}$ \\
\hline Coordinate GK Y (D-48) & $\mathrm{m}$ & $\begin{array}{l}\text { N } \\
\text { مे } \\
\infty \\
\text { 寸 }\end{array}$ & $\begin{array}{l}\text { 尺े } \\
\text { के } \\
\text { Oे }\end{array}$ & $\begin{array}{l}\stackrel{D}{J} \\
\stackrel{\sim}{\forall}\end{array}$ & 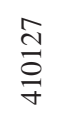 & $\begin{array}{l}\stackrel{ }{F} \\
\text { ন্ন }\end{array}$ & 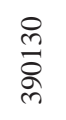 & $\begin{array}{l}\Re \\
\qquad \\
6 \\
\forall\end{array}$ & $\begin{array}{l}\text { ले } \\
\text { ్ֶ } \\
\text { ळి }\end{array}$ & 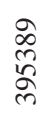 & $\begin{array}{l}\text { నె } \\
\text { ది } \\
\text { ळి }\end{array}$ & 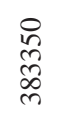 & $\begin{array}{l}\text { Jै } \\
-1 \\
\infty \\
\infty\end{array}$ & $\begin{array}{l}\text { Dิ } \\
\text { o. } \\
\text { ले }\end{array}$ & $\begin{array}{l}\text { ஜ } \\
\text { ஸू } \\
\text { ని }\end{array}$ & 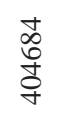 \\
\hline Coordinate GK X (D-48) & $\mathrm{m}$ & 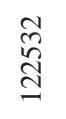 & 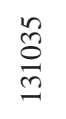 & $\begin{array}{l}\stackrel{m}{m} \\
\stackrel{m}{-}\end{array}$ & $\begin{array}{l}\text { ঠ } \\
\stackrel{-}{-1} \\
\stackrel{-}{-}\end{array}$ & $\begin{array}{l}\text { నू } \\
\text { న్ } \\
\stackrel{-}{-}\end{array}$ & $\begin{array}{l}\stackrel{\infty}{N} \\
\underset{N}{=} \\
\stackrel{n}{=}\end{array}$ & $\begin{array}{l}m \\
\text { DD } \\
0 \\
\stackrel{D}{+}\end{array}$ & $\begin{array}{l}\stackrel{+}{0} \\
\text { Jै } \\
\text { లె }\end{array}$ & 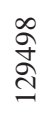 & 은 & $\begin{array}{l}\hat{N} \\
\infty \\
\stackrel{N}{=}\end{array}$ & 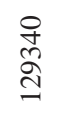 & 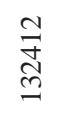 & 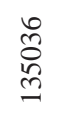 & 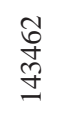 \\
\hline Author of the relevé (Avtor popisa) & & $\theta$ & $\theta$ & $\theta$ & $\theta$ & $\theta$ & ق & $\stackrel{4}{\Theta}$ & $\theta$ & $\theta$ & $\theta$ & 至 & 田 & $\theta$ & $\theta$ & ق \\
\hline
\end{tabular}

Character and differential species of the association (Značilnice in razlikovalnice asociacije)

VP Larix decidua

VP Larix decidua

VP Larix decidua

VP Larix decidua

VP Larix decidua

EP Rhododendron hirsutum

EP Rhodothamnus chamaecistus

AT Valeriana saxatilis

AT Primula auricula

AT Carex brachystachys

$\begin{array}{llllllllllllllll}\text { E3b } & 4 & 4 & 4 & 3 & 3 & 4 & 4 & 4 & 3 & 3 & 4 & 4 & 3 & 2 & 3 \\ \text { E3a } & 1 & 2 & + & 1 & . & + & + & 1 & 1 & 1 & 1 & 1 & . & . & . \\ \text { E2b } & 1 & 1 & 1 & 1 & 1 & + & 1 & 1 & + & . & . & 1 & . & . & + \\ \text { E2a } & . & + & 1 & + & 1 & . & + & . & . & . & + & + & . & + & . \\ \text { E1 } & + & . & + & . & . & + & + & . & . & . & + & . & . & . & . \\ \text { E2a } & 3 & 2 & 2 & 1 & 3 & 1 & + & + & 1 & . & 1 & 2 & 2 & 3 & 4 \\ \text { E1 } & 1 & 1 & 3 & 2 & 1 & 1 & + & + & 1 & + & + & 2 & 1 & + & 1 \\ \text { E1 } & + & + & + & 1 & + & + & . & . & + & . & + & + & 1 & 1 & . \\ \text { E1 } & . & . & + & + & . & + & . & . & . & . & + & + & + & . & . \\ \text { E1 } & . & . & . & \text { r } & . & . & . & . & . & . & + & . & + & . & .\end{array}$


Tabela 9: Subalpinski macesnov gozd (Rhodothamno-Laricetum) z zeleno jelšo

\begin{tabular}{|c|c|c|c|c|c|c|c|c|c|c|c|c|c|c|c|c|c|c|c|c|c|c|c|c|c|}
\hline 16 & 17 & 18 & 19 & 20 & 21 & 22 & 23 & 24 & 25 & 26 & 27 & 28 & 29 & 30 & 31 & 32 & 33 & 34 & 35 & 36 & 37 & 38 & 39 & 40 & \\
\hline $\begin{array}{l}\text { Oे } \\
\text { o } \\
\text { ㅎ․ }\end{array}$ & $\begin{array}{l}\stackrel{+}{ } \\
\stackrel{2}{+} \\
\stackrel{+}{\sim}\end{array}$ & 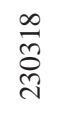 & 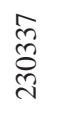 & $\begin{array}{l}\text { Oे } \\
\text { dे } \\
\text { ָे }\end{array}$ & $\begin{array}{l}\stackrel{\triangleright}{ } \\
\hat{0} \\
\end{array}$ & $\begin{array}{l}\text { Oे } \\
\text { o̊ } \\
\stackrel{+}{\sim}\end{array}$ & $\begin{array}{l}\stackrel{\infty}{0} \\
\stackrel{\infty}{O} \\
\stackrel{+}{\sim}\end{array}$ & 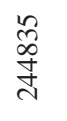 & 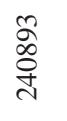 & 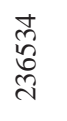 & $\begin{array}{l}\stackrel{\text { I }}{\Im} \\
\underset{\sim}{\sim}\end{array}$ & 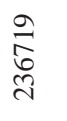 & 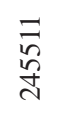 & 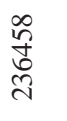 & $\begin{array}{l}\overrightarrow{0} \\
\mathbb{0} \\
\text { N }\end{array}$ & 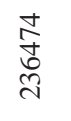 & 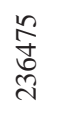 & \begin{tabular}{l}
$\mathscr{Q}$ \\
\multirow{0}{0}{} \\
సె
\end{tabular} & 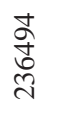 & 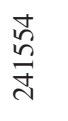 & 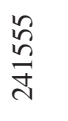 & 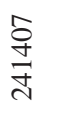 & 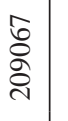 & 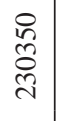 & \\
\hline 吕 & $\stackrel{̊}{\stackrel{2}{\beth}}$ & 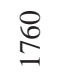 & $\stackrel{P}{\stackrel{R}{A}}$ & $\begin{array}{l}\stackrel{0}{ } \\
\stackrel{\leftrightarrow}{\sim}\end{array}$ & ભి & શ & 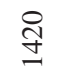 & 吕 & $\stackrel{0}{0}$ & $\stackrel{尺}{\stackrel{2}{I}}$ & $\begin{array}{l}\stackrel{\overbrace{}}{\beth} \\
ન\end{array}$ & $\begin{array}{l}\stackrel{D}{\mathbb{I}} \\
\underset{\sim}{*}\end{array}$ & $\stackrel{尺}{\stackrel{P}{I}}$ & 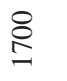 & ஜ્ఠ్ర్త్ & $\stackrel{尺}{\stackrel{P}{I}}$ & 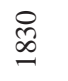 & 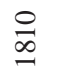 & શ્? & ્ָరి & 苞 & $\stackrel{\circ}{\underset{\exists}{\Xi}}$ & $\stackrel{ }{\underset{\exists}{\exists}}$ & $\stackrel{8}{\stackrel{1}{-1}}$ & \\
\hline $\mathrm{N}$ & $\mathrm{N}$ & NW & NE & NE & SW I & $\mathrm{NE}$ & $\mathrm{N}$ & $\mathrm{N}$ & $\mathrm{N}$ & NE & SW & $\mathrm{N}$ & NW & NE & NE & $\mathrm{N}$ & $\mathrm{N}$ & $\mathrm{N}$ & $\mathrm{N}$ & $\mathrm{N}$ & $\mathrm{N}$ & NW I & $\mathrm{NE}$ & NE & \\
\hline 40 & 45 & 30 & 45 & 50 & 35 & 40 & 35 & 40 & 45 & 35 & 30 & 20 & 35 & 35 & 40 & 45 & 45 & 45 & 40 & 45 & 40 & 40 & 50 & 35 & \\
\hline DA & $\mathrm{D}$ & $\mathrm{DA}$ & DA & DA & DA & D & DA & D & $\mathrm{D}$ & DA & A & DA & DA & D & $\mathrm{D}$ & $\mathrm{DA}$ & DA & DA & $\mathrm{AL}$ & D & $\mathrm{D}$ & A & $\mathrm{G}$ & G & \\
\hline $\mathrm{Re}$ & $\mathrm{Re}$ & $\mathrm{Re}$ & $\mathrm{Re}$ & $\mathrm{Re}$ & $\mathrm{Re}$ & $\mathrm{Re}$ & $\mathrm{Re}$ & $\mathrm{Re}$ & $\mathrm{Re}$ & $\mathrm{Re}$ & $\mathrm{Re}$ & $\mathrm{Re}$ & $\mathrm{Re}$ & $\mathrm{Re}$ & $\mathrm{Re}$ & $\mathrm{Re}$ & $\mathrm{Re}$ & $\mathrm{Re}$ & $\mathrm{Re}$ & $\mathrm{Re}$ & $\mathrm{Re}$ & $\mathrm{Re}$ & K & Ev & \\
\hline 5 & 10 & 10 & 10 & 5 & 20 & 10 & 30 & 10 & 20 & 20 & 20 & 50 & 20 & 5 & 5 & 10 & 15 & 20 & 5 & 20 & 20 & 30 & 20 & 0 & \\
\hline 50 & 60 & 40 & 50 & 50 & 50 & 50 & 50 & 70 & 60 & 40 & 60 & 20 & 40 & 60 & 60 & 40 & 40 & 50 & 60 & 40 & 40 & 70 & 50 & 50 & \\
\hline 10 & 10 & 10 & & 2 & 5 & 5 & 10 & 10 & 10 & 10 & 10 & & 10 & 5 & 5 & 5 & 5 & 5 & 5 & 5 & 5 & 20 & 10 & & \\
\hline 60 & 70 & 60 & 60 & 70 & 60 & 90 & 60 & 50 & 60 & 40 & 60 & 60 & 70 & 30 & 30 & 50 & 50 & 20 & 20 & 60 & 50 & 40 & 80 & 50 & \\
\hline 80 & 80 & 90 & 80 & 80 & 70 & 80 & 70 & 70 & 80 & 80 & 80 & 50 & 80 & 90 & 90 & 80 & 80 & 70 & 90 & 80 & 80 & 70 & 80 & 90 & \\
\hline 40 & 20 & 15 & 10 & 1 & 10 & 10 & 30 & 40 & 10 & 5 & 10 & 20 & 10 & 5 & 5 & 5 & 5 & 10 & 5 & 40 & 20 & 20 & 20 & 5 & \\
\hline 44 & 62 & 70 & 75 & 78 & 74 & 56 & 75 & 54 & 53 & 87 & 78 & 79 & 48 & 951 & 115 & 90 & 88 & 75 & 66 & 67 & 82 & 78 & 83 & 57 & \\
\hline 200 & 400 & 400 & 400 & 400 & 4004 & 400 & 400 & 400 & 400 & 400 & 400 & 200 & 200 & 400 & 400 & 400 & 400 & 400 & 400 & 400 & 4004 & 4002 & $200 \mid 4$ & 400 & \\
\hline 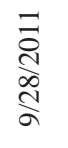 & 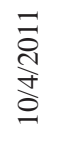 & $\begin{array}{l}\stackrel{8}{\circ} \\
\stackrel{1}{N} \\
\underset{N}{N}\end{array}$ & 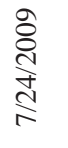 & 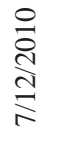 & $\begin{array}{l}\stackrel{\circ}{\stackrel{H}{N}} \\
\frac{N}{\sigma}\end{array}$ & 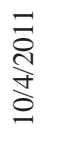 & 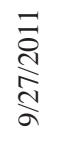 & 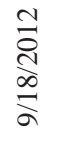 & 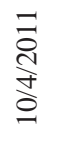 & $\begin{array}{l}0 \\
\stackrel{1}{1} \\
\stackrel{\Lambda}{\Delta} \\
\infty\end{array}$ & 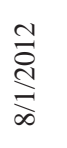 & 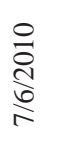 & 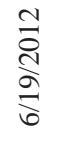 & 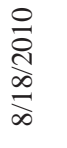 & $\begin{array}{l}\stackrel{0}{\circ} \\
\stackrel{\circ}{N} \\
\underset{\infty}{\infty} \\
\stackrel{\infty}{1}\end{array}$ & $\begin{array}{l}\stackrel{0}{1} \\
\stackrel{N}{1} \\
\stackrel{1}{1} \\
\infty\end{array}$ & 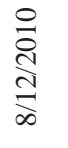 & 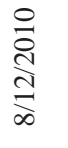 & 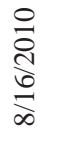 & $\frac{\vec{\sim}}{\stackrel{\vec{N}}{\sigma}}$ & 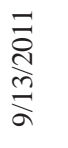 & 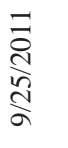 & 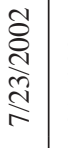 & 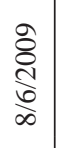 & \\
\hline 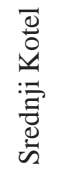 & 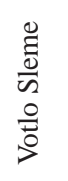 & 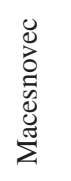 & $\begin{array}{l}\frac{5}{5} \\
\cdot \frac{7}{5} \\
\frac{\pi}{5} \\
5\end{array}$ & 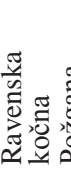 & 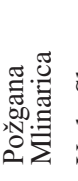 & 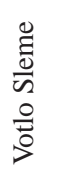 & 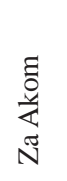 & $\begin{array}{l}\stackrel{0}{u} \\
\text {, } \\
0\end{array}$ & 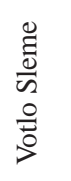 & $\frac{\tilde{E}}{\omega}$ & 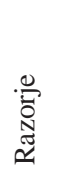 & 尝 & 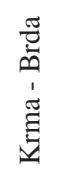 & 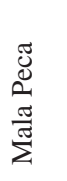 & 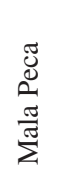 & 营 & 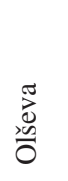 & 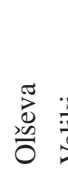 & 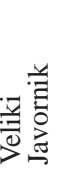 & 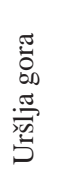 & 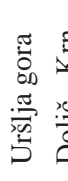 & 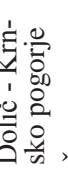 & 苟 & 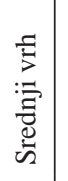 & \\
\hline 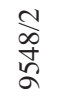 & 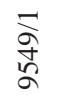 & $\begin{array}{l}\frac{m}{o r} \\
\text { 岁 }\end{array}$ & $\begin{array}{l}\overrightarrow{1} \\
\text { 守 }\end{array}$ & 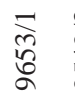 & $\begin{array}{l}m \\
\stackrel{m}{g} \\
\stackrel{9}{o}\end{array}$ & $\begin{array}{l}\overrightarrow{7} \\
\text { ఫे } \\
\text { 吕 }\end{array}$ & $\begin{array}{l}\stackrel{-1}{\sigma} \\
\text { 宫 }\end{array}$ & $\begin{array}{l}\stackrel{+}{f} \\
\text { 吕 }\end{array}$ & 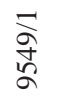 & $\begin{array}{l}m \\
\infty \\
\infty \\
ٌ \\
ٌ\end{array}$ & $\begin{array}{l}\frac{m}{\sigma} \\
\text { dै } \\
\mathscr{\sigma}\end{array}$ & $\begin{array}{l}\frac{1}{S} \\
\stackrel{T}{\sigma} \\
\sigma\end{array}$ & 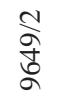 & 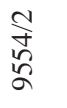 & $\begin{array}{l}\mathcal{N} \\
\text { 岕 } \\
\text { 呙 }\end{array}$ & $\begin{array}{l}\vec{f} \\
\text { 员 } \\
\text { 员 }\end{array}$ & 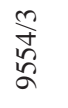 & $\begin{array}{l}\text { 案 } \\
\text { 员 }\end{array}$ & 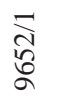 & $\begin{array}{l}\text { N } \\
\text { 㟧 } \\
\text { 物 }\end{array}$ & 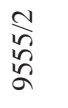 & 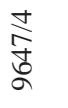 & 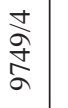 & $\begin{array}{l}m \\
\stackrel{n}{\prime} \\
\text { 吕 }\end{array}$ & \\
\hline $\begin{array}{l}\stackrel{8}{0} \\
\text { Oे } \\
\forall\end{array}$ & $\begin{array}{l}\vec{D} \\
\stackrel{0}{ } \\
m \\
\vec{\gamma}\end{array}$ & $\begin{array}{l}\vec{F} \\
\text { 吕 } \\
F\end{array}$ & $\begin{array}{l}\vec{p} \\
\text { 员 } \\
\text { F }\end{array}$ & $\begin{array}{l}\text { o } \\
\text { f } \\
\text { to }\end{array}$ & $\begin{array}{l}\text { 守 } \\
\stackrel{+}{\forall} \\
\forall\end{array}$ & $\begin{array}{l}\text { ळે } \\
\text { ले } \\
\forall\end{array}$ & 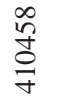 & 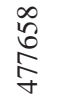 & $\begin{array}{l}\mathscr{m} \\
\infty \\
\infty \\
\dddot{m} \\
\vec{\gamma}\end{array}$ & $\begin{array}{l}\text { 尺̊ } \\
\text { 人ิ } \\
\text { Oे }\end{array}$ & 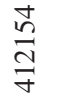 & 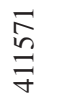 & 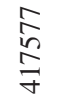 & 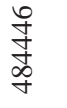 & 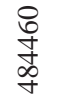 & 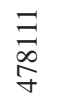 & 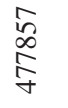 & 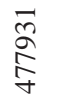 & 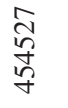 & 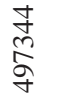 & 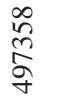 & $\begin{array}{l}\text { 吕 } \\
\text { 岗 } \\
\text { ले }\end{array}$ & $\begin{array}{l}\text { 今ू } \\
\text { ने } \\
\vec{\forall}\end{array}$ & 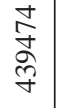 & \\
\hline 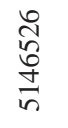 & 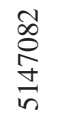 & 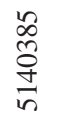 & $\begin{array}{l}\text { 昌 } \\
\text { 号 } \\
\text { ڤn }\end{array}$ & 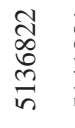 & $\begin{array}{l}\vec{\nabla} \\
\vec{\nabla} \\
\vec{b}\end{array}$ & 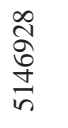 & 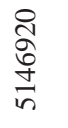 & 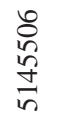 & 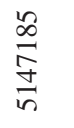 & 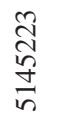 & 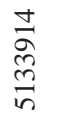 & 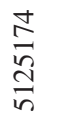 & 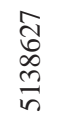 & 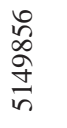 & 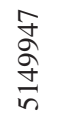 & 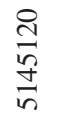 & 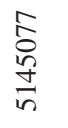 & $\begin{array}{l}\text { Nิ } \\
\text { 㝵 } \\
\text { ڤn }\end{array}$ & 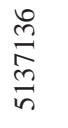 & 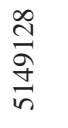 & 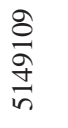 & 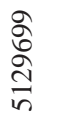 & $\begin{array}{l}\stackrel{\bullet}{\Xi} \\
\vec{\Xi} \\
\vec{\Xi}\end{array}$ & $\begin{array}{l}\stackrel{\mathcal{Y}}{\mathcal{H}} \\
\stackrel{\widetilde{J}}{\vec{H}}\end{array}$ & \\
\hline$\Theta$ & $\ominus$ & $\begin{array}{l}\text { 离 } \\
\hat{\Omega}\end{array}$ & 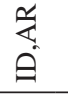 & $\begin{array}{l}\sum_{4}^{2} \\
\qquad\end{array}$ & $\begin{array}{l}\sum_{k}^{n} \\
\overbrace{1}^{2}\end{array}$ & $\ominus$ & 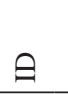 & $\begin{array}{l}e_{k} \\
\hat{=}\end{array}$ & $\theta$ & Ө & Я & Ө & & $\begin{array}{l}\sum_{k}^{\infty} \\
\overbrace{1}\end{array}$ & 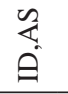 & 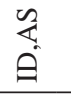 & $\begin{array}{l}\sum_{k}^{n} \\
\underbrace{2}_{1}\end{array}$ & $\begin{array}{l}\sum_{k}^{n} \\
\hat{=}\end{array}$ & $\begin{array}{l}\sum_{k}^{n} \\
\hat{=}\end{array}$ & 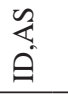 & $\begin{array}{l}\sum_{k} \\
\underbrace{}_{1}\end{array}$ & @ & a & 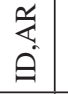 & Pr. Fr. \\
\hline
\end{tabular}

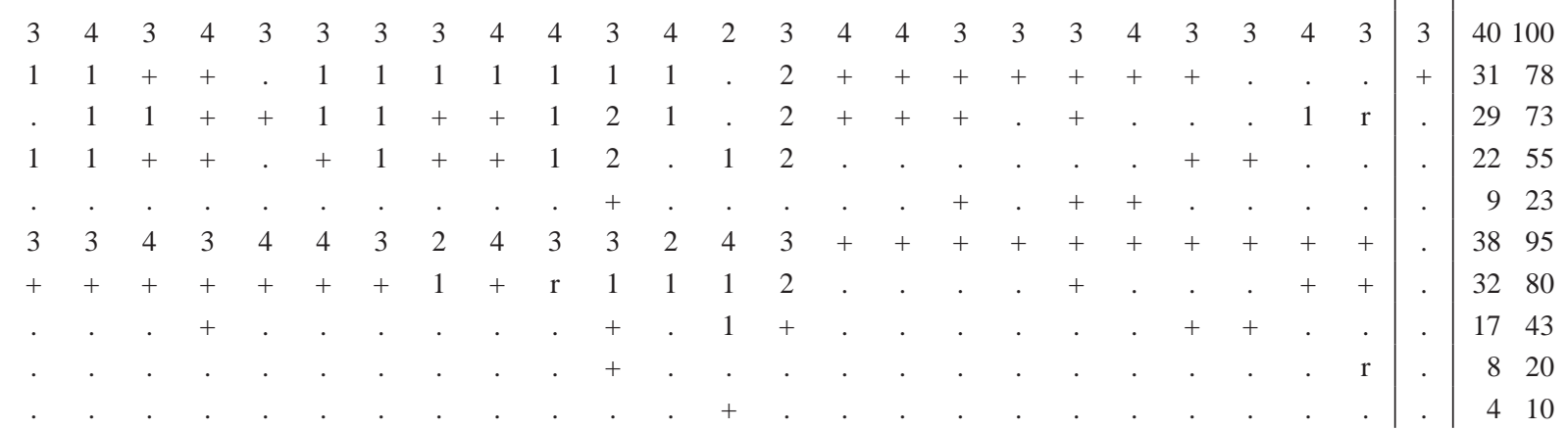


Number of relevé (Zaporedna številka popisa)

$\begin{array}{lllllllllllllll}1 & 2 & 3 & 4 & 5 & 6 & 7 & 8 & 9 & 10 & 11 & 12 & 13 & 14 & 15\end{array}$

\section{Geographical differential species (Geografske razlikovalne vrste)}

VP Homogyne sylvestris

AT Paederota lutea

AF Anemone trifolia

ES Laserpitium peucedanoides

TR Astrantia carniolica

AT Campanula carnica

Differential species of lower units (Razlikovalne vrste nižjih enot)

BA Alnus viridis
BA Alnus viridis
FS Luzula nivea

MuA Stellaria nemorum

VP Vaccinio-Piceetea

Vaccinium myrtillus

Luzula sylvatica

Vaccinium vitis-idaea

Valeriana tripteris

Polystichum lonchitis

Oxalis acetosella

Calamagrostis villosa

Clematis alpina

Dryopteris dilatata

Rosa pendulina

Homogyne alpina

Picea abies

Picea abies

Picea abies

Picea abies

Picea abies

Lycopodium annotinum

Phegopteris connectilis

Solidago virgaurea

Gymnocarpium dryopteris

Saxifraga cuneifolia

Huperzia selago

Melampyrum sylvaticum

Aposeris foetida

Hieracium murorum

Lonicera caerulea

Luzula luzuloides subsp. luzuloides

Veronica urticifolia

Maianthemum bifolium

Gentiana asclepiadea

Lonicera nigra

Dryopteris expansa

Calamagrostis arundinacea

Abies alba

Abies alba

Abies alba

Abies alba

Pyrola rotundifolia

E2a

E1

E1

E3b

E3a

E2b

E1

$\mathrm{E} 1++$

E1 +

E1

E2a

E1

E1

E2a

E1

E1 23

E3 $r$

E2b

E2a

E1

E1

$\begin{array}{lllllllllllllllll}\mathrm{E} 1 & 1 & . & 1 & + & + & + & + & + & . & . & \mathrm{r} & . & + & . & . \\ \mathrm{E} 1 & + & + & . & 1 & . & 1 & 1 & 1 & 1 & + & 1 & + & 1 & + & + \\ \mathrm{E} 1 & . & . & . & . & . & + & + & + & + & . & + & 1 & + & + & + \\ \mathrm{E} 1 & 1 & . & + & 1 & . & . & . & . & + & + & + & . & + & + & . \\ \mathrm{E} 1 & . & + & 1 & + & + & . & . & . & . & . & . & . & . & . & . \\ \mathrm{E} 1 & . & . & . & . & . & . & . & . & . & . & . & . & & & . & \\ \end{array}$

$\begin{array}{llllllllllllllll}\text { E2b } & 1 & 1 & 1 & 1 & 1 & 1 & 1 & 1 & 2 & 1 & 1 & 1 & 2 & 1 & 3\end{array}$

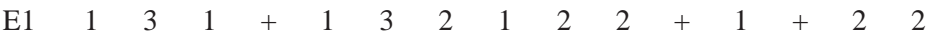

$\mathrm{E} 11111+\begin{array}{llllllll}1 & 1 & 1 & 1 & 1 & 1 & + & +\end{array}$

$\mathrm{E} 1+21+++1++++++13$

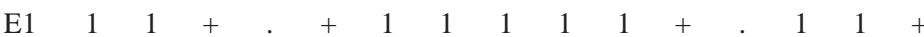

$\mathrm{E} 1+1+1+++++1++.+$.

$\mathrm{E} 1+++1++1 .+1+1 .+$

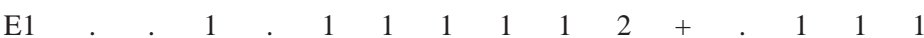

$\mathrm{E} 1+1+++\begin{array}{lllllllll} & 2 & 1 & 1 & 1 & 1 & 1 & 1 & 1\end{array}$

$\mathrm{E} 1+++++++\mathrm{r} \cdot+$.

$\mathrm{E} 2 \mathrm{a}+++1+++++++{ }_{+}+1+$

$\mathrm{E} 1++$. $+11+++$. $+1+$

$\mathrm{E} 2 \mathrm{a}++++$. 1 . . ${ }^{+}+$. $\mathrm{r}+$

$\mathrm{E} 1+11+++1+\ldots+1+24$

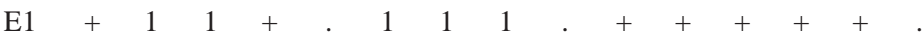

$\mathrm{E} 111+1+++1+1++++$.

$\mathrm{E} 111++1$. $1+++$. ++

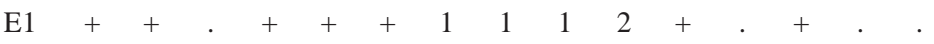

$\mathrm{E} 1++$

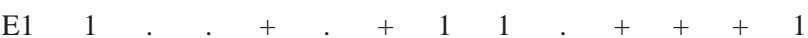




\begin{tabular}{|c|c|c|c|c|c|c|c|c|c|c|c|c|c|c|c|c|c|c|c|c|c|c|c|c|c|c|}
\hline 16 & 17 & 18 & 19 & 20 & 21 & 22 & 23 & 24 & 25 & 26 & 27 & 28 & 29 & 30 & 31 & 32 & 33 & 34 & 35 & 36 & 37 & 38 & 39 & 40 & Pr. & Fr. \\
\hline 1 & . & + & . & 1 & + & . & . & 1 & 1 & . & . & . & . & + & 1 & 1 & 1 & + & . & 1 & 1 & . & + & . & 23 & 58 \\
\hline . & + & + & 1 & + & + & . & + & 1 & + & + & 1 & + & . & + & + & + & + & 1 & 1 & + & . & + & 1 & . & 33 & 83 \\
\hline 1 & 1 & . & + & . & 1 & 1 & 1 & . & 1 & . & . & 1 & . & . & . & . & . & . & . & . & . & . & . & . & 17 & 43 \\
\hline+ & . & + & . & + & + & . & + & . & . & + & . & . & . & . & . & . & . & . & . & . & . & + & + & . & 16 & 40 \\
\hline . & . & . & . & + & . & . & . & . & . & . & . & . & . & . & . & . & . & . & . & . & . & . & . & . & 5 & 13 \\
\hline . & . & . & . & . & . & . & . & . & . & . & . & . & . & . & . & . & . & . & . & . & . & . & + & . & 2 & 5 \\
\hline 3 & 3 & 2 & 2 & 2 & 1 & 1 & 2 & 1 & 2 & 1 & 1 & 1 & 1 & 1 & 1 & 3 & 3 & 1 & 2 & 4 & 3 & 2 & 4 & 3 & & 100 \\
\hline . & . & + & . & . & . & . & . & + & . & . & . & + & . & . & . & . & . & . & + & . & . & . & . & . & 8 & 20 \\
\hline . & . & . & . & . & . & . & . & . & . & . & . & . & . & . & . & . & . & . & . & . & . & + & . & . & 8 & 20 \\
\hline . & . & . & . & . & . & . & . & . & . & . & . & . & . & 1 & + & 1 & 1 & 1 & 1 & 1 & + & . & . & 2 & 9 & 23 \\
\hline 3 & 3 & 3 & 1 & 1 & 2 & 2 & 3 & 3 & . & 3 & 3 & 3 & 1 & 1 & 1 & 3 & 2 & 1 & 1 & + & 1 & 2 & . & + & 38 & 95 \\
\hline 1 & 1 & 1 & + & 1 & + & 1 & 1 & + & 2 & 1 & 1 & . & 1 & 2 & 2 & 1 & 1 & 1 & 3 & 2 & 2 & 2 & . & 1 & 37 & 93 \\
\hline+ & 2 & 1 & 2 & 2 & 2 & 2 & 2 & 3 & 2 & + & 1 & 2 & + & + & + & + & + & + & . & + & + & + & . & . & 37 & 93 \\
\hline 1 & 1 & 1 & 1 & + & + & 1 & 1 & + & 1 & 1 & 1 & + & + & + & + & + & 1 & 1 & + & + & 1 & 1 & + & . & 37 & 93 \\
\hline+ & 1 & + & + & 1 & + & 1 & + & + & + & 1 & 1 & + & . & + & + & + & + & + & + & 1 & + & 1 & + & + & 37 & 93 \\
\hline 1 & 1 & 1 & 1 & 1 & + & 1 & 1 & 1 & . & + & . & 1 & . & 1 & 1 & 1 & 1 & 1 & 1 & 1 & 1 & 1 & + & . & 34 & 85 \\
\hline 3 & 3 & 1 & 1 & 1 & 1 & 2 & 3 & 1 & 3 & 1 & 1 & 1 & . & 2 & 1 & 1 & 1 & 2 & 1 & . & 1 & 1 & . & 1 & 33 & 83 \\
\hline . & + & 1 & + & 1 & 1 & 1 & 1 & 1 & 1 & 1 & + & 1 & . & . & + & . & + & + & . & . & + & + & + & . & 33 & 83 \\
\hline+ & + & + & 1 & + & + & + & 1 & . & + & + & + & + & . & 1 & + & 1 & 2 & 1 & 1 & + & + & 1 & + & 1 & 32 & 80 \\
\hline . & + & + & . & + & + & + & 1 & . & . & 1 & 2 & . & . & + & + & . & . & . & . & + & + & + & + & . & 29 & 73 \\
\hline 1 & 1 & 1 & + & + & . & + & + & 1 & . & + & + & 1 & + & . & . & + & . & . & + & . & + & + & . & . & 28 & 70 \\
\hline . & . & . & . & . & $\mathrm{r}$ & . & + & . & . & . & . & . & . & + & + & . & + & . & + & + & . & . & $\mathrm{r}$ & + & 16 & 40 \\
\hline+ & . & . & . & . & . & + & . & 1 & + & . & $r$ & . & . & 1 & + & + & + & . & . & + & + & + & $\mathrm{r}$ & . & 19 & 48 \\
\hline . & . & . & . & + & + & + & + & 1 & + & + & . & 1 & . & + & + & . & . & . & 1 & + & + & + & + & . & 24 & 60 \\
\hline . & + & + & + & + & + & . & + & 1 & + & . & . & . & + & . & + & + & + & + & 1 & + & + & + & + & + & 28 & 70 \\
\hline . & . & . & . & . & . & . & . & . & . & $\cdot$ & . & + & . & . & . & . & . & . & . & . & . & . & . & . & 7 & 18 \\
\hline 1 & 2 & 1 & 1 & 1 & + & + & 1 & 1 & 2 & . & 1 & 2 & 1 & . & . & . & . & . & . & + & + & . & . & . & 28 & 70 \\
\hline 1 & + & . & + & + & + & + & + & + & + & + & . & 1 & . & . & . & . & . & . & 1 & . & 1 & . & + & + & 27 & 68 \\
\hline . & . & + & + & . & + & . & 1 & . & . & + & 1 & + & . & . & . & + & . & . & . & . & . & + & + & + & 25 & 63 \\
\hline+ & . & . & + & . & + & . & + & 1 & . & . & + & + & . & + & . & + & + & . & + & . & + & . & . & . & 24 & 60 \\
\hline . & . & . & . & + & . & . & . & + & + & + & . & 1 & . & + & 1 & + & + & + & 1 & . & . & 1 & . & . & 23 & 58 \\
\hline . & + & . & + & + & . & . & + & . & + & . & + & + & + & . & . & . & . & . & + & . & + & . & . & . & 22 & 55 \\
\hline . & . & + & + & + & . & . & . & + & . & + & + & + & . & + & + & + & + & + & . & . & + & . & . & . & 21 & 53 \\
\hline 1 & 1 & 1 & + & 1 & + & 1 & . & + & . & . & . & . & + & + & . & + & + & . & 1 & . & . & . & . & 1 & 19 & 48 \\
\hline . & . & + & + & + & + & + & . & . & . & . & + & + & . & . & + & . & . & + & . & . & . & 1 & . & . & 19 & 48 \\
\hline . & + & + & + & . & + & + & + & . & . & + & 2 & 1 & . & . & . & . & . & . & . & . & . & + & . & . & 19 & 48 \\
\hline . & . & . & . & . & . & . & . & + & . & . & + & . & . & + & + & 1 & 1 & 1 & + & 1 & 1 & 1 & + & + & 16 & 40 \\
\hline . & . & . & . & + & . & + & . & . & + & . & + & . & . & . & . & . & . & . & . & . & . & 1 & + & . & 15 & 38 \\
\hline . & . & . & . & . & + & . & + & . & . & . & 1 & + & . & . & . & . & . & . & . & . & . & + & . & . & 14 & 35 \\
\hline+ & . & . & . & . & . & . & . & . & . & . & . & + & . & + & 1 & . & . & . & . & . & . & . & . & . & 13 & 33 \\
\hline . & . & . & + & + & . & . & + & . & . & . & . & . & . & + & . & . & + & . & . & . & + & + & . & + & 13 & 33 \\
\hline . & . & + & . & . & . & . & . & . & . & . & . & + & . & 1 & + & + & + & . & . & . & . & + & . & + & 13 & 33 \\
\hline . & . & . & . & . & . & + & + & . & . & . & . & + & + & . & . & . & . & . & + & . & . & 1 & 3 & . & 12 & 30 \\
\hline . & . & . & . & . & . & . & $\mathrm{r}$ & . & $r$ & . & . & . & . & . & . & . & . & . & . & . & . & . & . & . & 3 & 8 \\
\hline . & $r$ & . & . & + & . & . & + & . & . & . & . & 1 & . & . & . & . & . & . & . & . & . & . & . & . & 6 & 15 \\
\hline . & . & . & . & + & . & . & . & + & . & . & . & + & . & . & . & . & . & . & . & . & . & . & . & . & 5 & 13 \\
\hline . & . & . & . & . & . & . & . & . & . & . & . & + & . & . & . & . & . & . & . & . & . & . & . & . & 2 & 5 \\
\hline . & . & + & + & . & . & . & . & . & . & . & . & + & . & . & . & . & . & . & . & . & . & . & . & . & 4 & 10 \\
\hline
\end{tabular}




\begin{tabular}{|c|c|c|c|c|c|c|c|c|c|c|c|c|c|c|c|c|}
\hline Number of relevé (Zaporedna številka popisa) & & 1 & 2 & 3 & 4 & 5 & 6 & 7 & 8 & 9 & 10 & 11 & 12 & 13 & 14 & 15 \\
\hline Luzula luzulina & E1 & . & . & . & . & . & . & + & . & . & . & . & . & . & . & . \\
\hline Luzula pilosa & E1 & . & . & . & . & . & . & . & . & . & . & . & . & . & . & . \\
\hline Moneses uniflora & E1 & . & . & . & . & . & . & . & . & + & . & . & . & . & . & . \\
\hline Orthilia secunda & E1 & . & . & . & . & . & . & + & . & . & . & . & . & . & . & . \\
\hline Pyrola minor & E1 & . & . & . & . & . & . & . & . & . & . & . & . & . & . & + \\
\hline Ajuga pyramidalis & E1 & . & . & . & . & . & . & . & . & . & . & . & . & . & . & . \\
\hline Listera cordata & E1 & . & . & . & . & . & . & . & . & . & . & . & . & . & . & . \\
\hline Luzula luzuloides subsp. rubella & E1 & . & . & . & . & . & . & . & . & . & . & . & . & . & . & . \\
\hline Pyrola chlorantha & E1 & . & . & . & . & . & . & . & . & . & . & . & . & . & . & $\mathrm{r}$ \\
\hline Erico-Pinetea & & & & & & & & & & & & & & & & \\
\hline Rubus saxatilis & E1 & 1 & 1 & 1 & 1 & 1 & + & + & 1 & 1 & 2 & + & + & + & . & + \\
\hline Pinus mugo & E2b & . & . & + & . & + & + & + & 1 & + & . & + & 2 & + & 4 & 4 \\
\hline Calamagrostis varia & E1 & . & 2 & 1 & 2 & . & 1 & . & . & + & . & 1 & + & + & 1 & . \\
\hline Erica carnea & E1 & . & . & 2 & . & . & . & . & . & . & . & + & 1 & . & + & + \\
\hline Carex ornithopoda & E1 & . & . & . & . & . & . & . & . & . & . & . & + & . & . & + \\
\hline Cirsium erisithales & E1 & + & . & . & + & . & . & . & . & . & + & + & + & . & . & . \\
\hline Aquilegia nigricans & E1 & . & + & . & + & . & . & . & . & + & . & . & . & . & . & . \\
\hline Juniperus alpina & E2a & . & . & . & . & . & . & . & . & . & . & . & . & $\mathrm{r}$ & . & . \\
\hline Arctostaphylos uva-ursi & E1 & . & . & . & . & . & . & . & . & . & . & . & . & . & + & . \\
\hline Galium austriacum & E1 & . & . & . & . & . & . & . & . & . & . & . & . & . & . & . \\
\hline Molinia caerulea subsp. arundinacea & E1 & . & . & . & . & . & . & . & . & . & . & . & . & . & . & . \\
\hline Aremonio-Fagion & & & & & & & & & & & & & & & & \\
\hline Dentaria enneaphyllos & E1 & + & . & . & . & + & . & 1 & . & . & . & . & + & . & . & . \\
\hline Knautia drymeia & E1 & $\mathrm{r}$ & . & . & . & . & . & . & . & . & . & . & . & . & . & . \\
\hline Helleborus niger & E1 & . & . & . & . & . & . & . & . & . & . & . & . & . & . & . \\
\hline Cardamine trifolia & E1 & . & . & . & + & . & . & . & . & . & . & . & . & . & . & . \\
\hline Cyclamen purpurascens & E1 & + & . & . & . & . & . & . & . & . & . & . & . & . & . & . \\
\hline Rhamnus fallax & E2 & . & . & . & . & . & . & . & . & + & . & . & . & . & . & . \\
\hline Fagetalia sylvaticae & & & & & & & & & & & & & & & & \\
\hline Daphne mezereum & E2a & $\mathrm{r}$ & . & + & . & + & . & . & . & . & + & + & . & . & . & $\mathrm{r}$ \\
\hline Melica nutans & E1 & + & . & + & + & + & 1 & + & . & . & . & + & . & + & . & . \\
\hline Galeobdolon flavidum & E1 & . & . & . & . & . & . & + & . & . & . & + & + & + & . & . \\
\hline Lonicera alpigena & E2a & . & 1 & + & + & . & . & + & + & . & . & 1 & . & . & . & . \\
\hline Poa nemoralis & E1 & . & . & . & . & . & . & + & . & . & . & + & . & . & . & . \\
\hline Mercurialis perennis & E1 & 1 & . & . & . & . & . & + & + & . & . & 1 & . & 1 & . & . \\
\hline Paris quadrifolia & E1 & + & . & . & . & + & . & + & . & . & + & + & + & . & . & . \\
\hline Dryopteris filix-mas & E1 & + & . & . & . & . & . & + & + & + & + & + & . & . & . & . \\
\hline Prenanthes purpurea & E1 & + & + & + & . & . & + & + & . & . & . & . & . & . & + & + \\
\hline Myosotis sylvatica & E1 & . & . & . & . & . & . & . & . & . & . & . & . & . & . & . \\
\hline Chrysosplenium alternifolium & E1 & . & . & . & . & . & . & . & . & . & + & . & . & . & . & . \\
\hline Fagus sylvatica & E3b & . & . & . & . & . & . & . & . & . & . & + & . & $\mathrm{r}$ & . & . \\
\hline Fagus sylvatica & E3a & + & $\mathrm{r}$ & + & . & . & + & . & . & . & . & + & . & . & . & . \\
\hline Fagus sylvatica & E2b & + & . & . & . & $\mathrm{r}$ & . & . & . & . & . & . & . & $\mathrm{r}$ & . & . \\
\hline Fagus sylvatica & E2a & . & + & + & . & . & . & . & . & . & . & . & . & . & . & . \\
\hline Fagus sylvatica & E1 & . & . & . & . & . & . & + & . & . & . & . & . & . & . & . \\
\hline Lilium martagon & E1 & . & . & . & + & . & . & . & . & . & . & $\mathrm{r}$ & + & . & . & . \\
\hline Epilobium montanum & E1 & . & . & . & . & . & . & . & . & + & . & . & . & . & . & . \\
\hline Acer pseudoplatanus & E3a & . & + & . & . & . & . & . & . & . & . & + & . & . & . & . \\
\hline Acer pseudoplatanus & $\mathrm{E} 2 \mathrm{~b}$ & . & . & . & . & . & . & . & . & . & . & + & . & . & . & . \\
\hline Acer pseudoplatanus & E2a & . & . & . & . & . & . & . & $\mathrm{r}$ & . & . & . & . & . & . & . \\
\hline Acer pseudoplatanus & E1 & . & . & + & + & . & . & . & + & . & . & . & . & . & . & \\
\hline
\end{tabular}




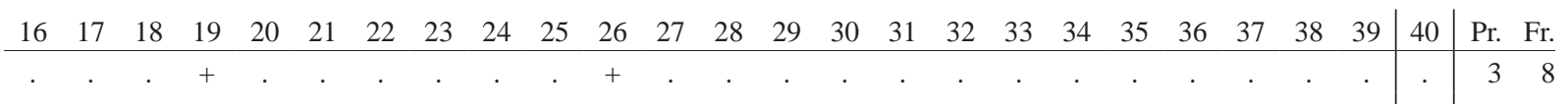

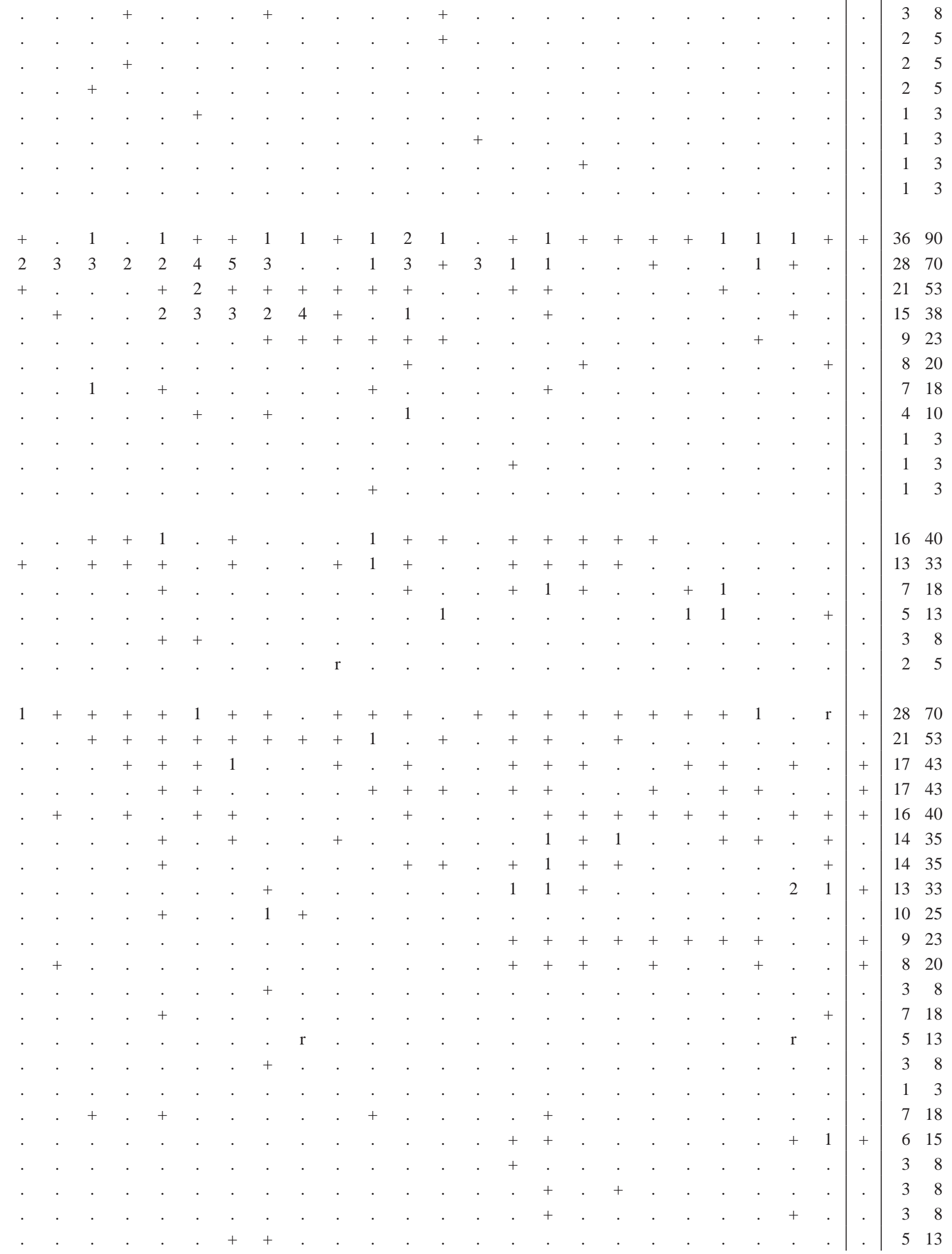




\begin{tabular}{|c|c|c|c|c|c|c|c|c|c|c|c|c|c|c|c|c|}
\hline Number of relevé (Zaporedna številka popisa) & & 1 & 2 & 3 & 4 & 5 & 6 & 7 & 8 & 9 & 10 & 11 & 12 & 13 & 14 & 15 \\
\hline Symphytum tuberosum & E1 & . & . & . & . & . & . & + & . & . & . & . & . & . & . & . \\
\hline Polystichum aculeatum & E1 & . & . & . & . & . & . & . & . & . & . & . & . & . & . & . \\
\hline Actaea spicata & E1 & . & . & . & . & . & + & . & . & . & + & . & . & . & . & . \\
\hline Galium laevigatum & E1 & . & . & . & + & . & + & . & + & . & . & . & . & . & . & . \\
\hline Adoxa moschatellina & E1 & . & . & . & . & . & . & . & . & . & . & . & . & . & . & . \\
\hline Festuca heterophylla & E1 & . & . & . & . & . & . & . & . & . & . & . & . & . & . & . \\
\hline Aruncus dioicus & E1 & . & . & . & r & . & . & . & . & . & . & . & . & . & . & . \\
\hline Campanula trachelium & E1 & . & . & . & . & . & . & . & . & . & . & . & . & . & . & . \\
\hline Euphorbia amygdaloides & E1 & . & . & . & . & . & . & . & . & . & . & . & . & . & . & . \\
\hline Laburnum alpinum & E3a & . & . & . & . & . & . & . & . & . & . & . & . & . & . & . \\
\hline Laburnum alpinum & E1 & . & . & . & . & . & . & . & . & . & . & . & . & . & . & . \\
\hline Mycelis muralis & E1 & . & . & . & . & . & . & . & . & . & . & . & . & . & . & . \\
\hline Phyteuma spicatum & E1 & . & . & . & . & . & . & . & . & . & . & . & . & . & . & . \\
\hline Ranunculus lanuginosus & E1 & . & . & . & . & . & . & . & . & . & . & . & . & . & . & . \\
\hline \multicolumn{17}{|l|}{ Quercetalia pubescentis } \\
\hline Sorbus aria & E2a & . & . & . & . & . & . & . & . & + & . & . & . & . & . & . \\
\hline Arabis turrita & E1 & . & . & . & . & . & . & . & . & . & . & . & . & . & . & . \\
\hline Convallaria majalis & E1 & . & . & . & . & . & . & . & . & . & . & + & . & . & . & . \\
\hline Primula veris subsp. columnae & E1 & . & . & . & . & . & . & . & . & . & . & $\mathrm{r}$ & . & . & . & . \\
\hline \multicolumn{17}{|l|}{ Querco-Fagetea } \\
\hline Hepatica nobilis & E1 & . & . & . & 1 & . & . & + & . & . & . & + & . & . & . & . \\
\hline Anemone nemorosa & E1 & 1 & + & . & 1 & . & . & + & . & . & . & . & . & . & . & . \\
\hline Carex digitata & E1 & . & . & . & . & + & + & . & . & . & . & + & . & . & . & . \\
\hline Dactylorhiza fuchsii & E1 & . & . & . & . & . & . & . & $\mathrm{r}$ & . & . & . & . & . & . & . \\
\hline Galium sylvaticum & E1 & . & . & . & . & . & . & . & . & . & . & . & . & . & . & . \\
\hline Ranunculus auricomus agg. & E1 & . & . & . & . & . & . & . & . & . & . & . & + & . & . & . \\
\hline Viola riviniana & E1 & . & . & . & . & . & . & . & . & . & . & . & . & . & . & . \\
\hline Betula pendula & EЗа & . & . & . & . & . & . & . & . & . & . & . & . & . & . & . \\
\hline \multicolumn{17}{|l|}{ Betulo carpaticae-Alnetea viridis } \\
\hline Salix appendiculata & E3a & . & . & . & . & . & . & . & . & + & . & . & . & . & . & . \\
\hline Salix appendiculata & E2b & 1 & 1 & + & + & + & . & . & . & + & 2 & 1 & + & 1 & + & . \\
\hline Salix appendiculata & E2a & . & . & + & . & + & . & . & . & . & . & + & . & 1 & + & . \\
\hline Salix appendiculata & E1 & . & & . & . & + & & . & . & . & . & . & . & . & + & . \\
\hline Sorbus chamaemespilus & E2 & + & 1 & 1 & . & 1 & . & + & + & 1 & + & . & + & + & + & 1 \\
\hline Salix glabra & E2 & . & + & . & . & & + & + & . & . & . & . & . & . & . & + \\
\hline Salix waldsteiniana & E2a & . & . & + & . & . & . & . & . & . & . & . & . & . & . & + \\
\hline Ribes alpinum & E2a & . & . & . & + & . & . & . & . & . & . & . & . & . & . & . \\
\hline \multicolumn{17}{|l|}{ Sambuco-Salicion capreae } \\
\hline Sorbus aucuparia & E3b & . & . & . & . & . & . & . & . & . & 1 & . & . & . & . & . \\
\hline Sorbus aucuparia & E3a & + & + & + & + & . & + & . & + & . & 1 & + & . & . & . & . \\
\hline Sorbus aucuparia & E2b & + & + & + & + & + & . & . & 1 & 1 & 1 & + & . & 1 & + & 1 \\
\hline Sorbus aucuparia & E2a & . & . & . & . & . & . & + & . & + & . & . & + & + & + & + \\
\hline Sorbus aucuparia & E1 & . & + & . & + & . & . & + & + & . & + & . & . & . & . & + \\
\hline Rubus idaeus & E2a & . & . & . & . & . & . & . & . & . & . & . & + & . & . & . \\
\hline Fragaria vesca & E1 & . & . & . & . & . & . & . & . & . & . & . & + & . & . & . \\
\hline Urtica dioica & E1 & . & . & . & . & . & . & . & + & + & + & . & . & . & . & . \\
\hline Galeopsis speciosa & E1 & $\mathrm{r}$ & . & . & . & . & . & . & + & . & . & . & . & . & . & . \\
\hline \multicolumn{17}{|l|}{ Mulgedio-Aconitetea } \\
\hline Viola biflora & E1 & + & + & 1 & 1 & 1 & 1 & 1 & 1 & + & 1 & 1 & 1 & 1 & + & + \\
\hline Geranium sylvaticum & E1 & + & + & + & . & + & . & + & + & + & 1 & . & + & + & + & + \\
\hline Polygonatum verticillatum & E1 & + & . & + & + & . & + & + & + & 1 & 1 & + & . & + & . & . \\
\hline
\end{tabular}




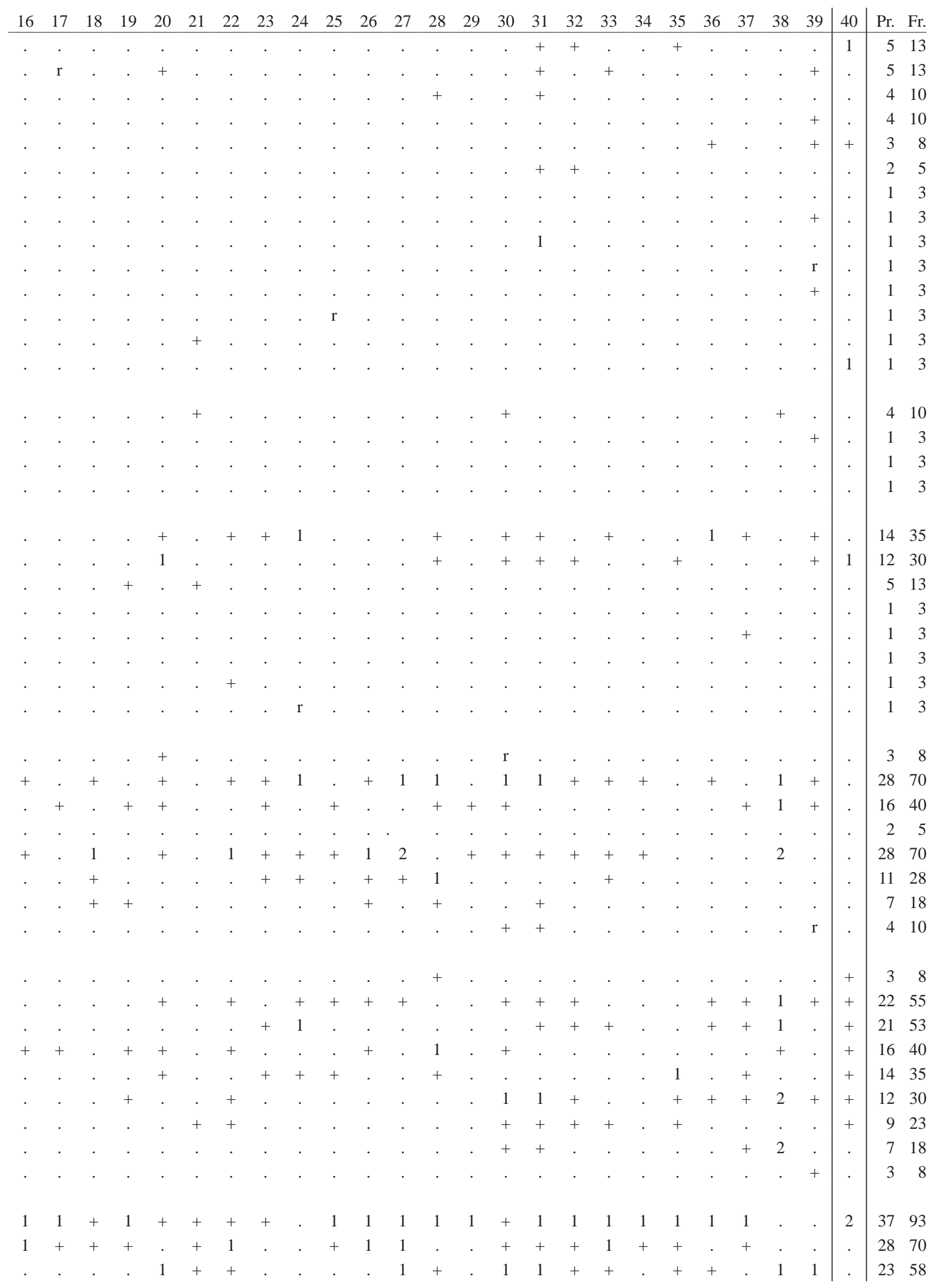




\begin{tabular}{|c|c|c|c|c|c|c|c|c|c|c|c|c|c|c|c|c|c|}
\hline & Number of relevé (Zaporedna številka popisa) & & 1 & 2 & 3 & 4 & 5 & 6 & 7 & 8 & 9 & 10 & 11 & 12 & 13 & 14 & 15 \\
\hline & Saxifraga rotundifolia & E1 & . & + & . & . & + & . & + & + & + & 2 & + & . & . & . & . \\
\hline & Athyrium filix-femina & E1 & + & . & . & . & + & + & + & + & + & 1 & + & + & . & . & . \\
\hline & Veratrum album s.lat. & E1 & + & 1 & . & + & . & . & . & + & + & . & 1 & 1 & . & . & + \\
\hline & Chaerophyllum hirsutum & E1 & . & . & . & . & . & . & + & + & + & + & . & . & . & + & . \\
\hline & Adenostyles alliariae & E1 & . & . & . & . & . & . & + & . & . & + & . & . & . & . & . \\
\hline & Ranunculus platanifolius & E1 & . & + & . & + & + & . & . & + & + & . & + & $\mathrm{r}$ & 1 & . & . \\
\hline & Aconitum lycoctonum subsp. ranunculifolium & E1 & . & . & . & + & + & . & . & + & 1 & + & + & + & . & . & . \\
\hline & Geum rivale & E1 & . & + & . & . & . & . & . & + & + & + & . & . & . & . & . \\
\hline & Hypericum maculatum & E1 & . & . & . & . & + & . & . & + & + & . & + & . & . & . & . \\
\hline & Phyteuma ovatum & E1 & + & + & . & . & + & + & + & . & . & . & $\mathrm{r}$ & . & . & . & . \\
\hline & Poa hybrida & E1 & . & . & . & . & + & . & . & . & . & . & . & . & . & . & . \\
\hline & Senecio ovatus & E1 & + & . & . & . & . & . & . & . & . & . & + & . & . & . & . \\
\hline & Senecio cacaliaster & E1 & + & . & . & . & . & . & . & + & . & . & . & + & . & . & . \\
\hline & Thalictrum aquilegiifolium & E1 & . & . & . & . & . & . & . & + & . & . & + & . & + & . & . \\
\hline & Doronicum austriacum & E1 & . & . & . & . & . & . & . & . & . & + & . & . & . & . & . \\
\hline & Primula elatior & E1 & . & . & . & . & . & . & . & . & . & + & . & . & . & . & . \\
\hline & Rumex arifolius & E1 & . & . & . & . & . & . & . & . & + & . & . & . & . & . & . \\
\hline & Aconitum angustifolium & E1 & . & . & . & . & . & . & . & . & + & . & . & + & . & . & . \\
\hline & Alchemilla xanthochlora & E1 & . & . & . & . & + & . & . & + & . & . & + & . & . & . & . \\
\hline & Crepis paludosa & E1 & + & . & . & . & . & . & + & . & . & . & . & . & . & . & . \\
\hline & Aconitum tauricum & E1 & . & . & . & . & . & . & . & . & . & . & . & . & . & . & . \\
\hline & Chaerophyllum villarsii & E1 & . & . & . & . & + & . & . & . & . & . & . & . & . & . & . \\
\hline & Cortusa matthioli & E1 & . & . & . & . & . & . & . & . & . & . & . & . & . & . & . \\
\hline & Epilobium alpestre & E1 & . & . & . & . & . & . & . & . & . & . & . & . & . & . & . \\
\hline & Peucedanum ostruthium & E1 & . & . & . & . & . & . & . & 1 & . & . & . & . & . & . & . \\
\hline & Alchemilla monticola & E1 & . & . & . & . & . & . & . & . & . & . & . & . & . & . & . \\
\hline & Cicerbita alpina & E1 & . & . & . & . & . & . & . & . & . & . & . & . & . & . & . \\
\hline & Heracleum montanum & E1 & . & . & . & . & . & . & . & . & . & . & . & . & . & . & . \\
\hline & Knautia longifolia & E1 & . & . & . & . & . & . & . & . & + & . & . & . & . & . & . \\
\hline & Milium effusum & E1 & . & . & . & . & . & . & . & . & . & . & . & . & . & . & . \\
\hline & Pedicularis recutita & E1 & . & . & . & . & . & . & . & . & . & . & . & . & . & . & . \\
\hline & Rumex alpinus & E1 & . & . & . & . & . & . & . & . & . & . & . & . & . & . & . \\
\hline & Scrophularia scopolii & E1 & . & . & . & . & . & . & . & . & . & . & . & . & . & . & . \\
\hline & Athyrium distentifolium & E1 & . & . & . & . & . & . & . & . & . & . & . & . & . & . & . \\
\hline ES & Elyno-Seslerietea & & & & & & & & & & & & & & & & \\
\hline & Sesleria caerulea subsp. calcaria & E1 & 2 & + & 2 & 3 & 1 & 2 & 2 & 1 & 1 & 1 & 2 & 1 & . & 1 & + \\
\hline & Aster bellidiastrum & E1 & 1 & . & + & 1 & + & 1 & + & . & + & + & + & + & + & . & . \\
\hline & Heliosperma alpestre & E1 & + & + & + & . & + & + & + & . & + & . & . & . & . & + & . \\
\hline & Astrantia bavarica & E1 & + & + & . & + & + & . & . & + & + & + & . & + & . & + & . \\
\hline & Polygonum viviparum & E1 & . & . & + & 1 & + & . & + & . & . & 1 & + & + & + & . & . \\
\hline & Carex ferruginea & E1 & . & . & + & . & 1 & . & . & . & . & . & . & . & . & . & . \\
\hline & Phyteuma orbiculare & E1 & + & . & . & . & . & . & . & . & . & . & . & . & . & . & . \\
\hline & Campanula witasekiana & E1 & . & . & . & . & . & . & . & . & . & . & . & . & . & . & . \\
\hline & Festuca calva & E1 & . & . & . & . & . & + & . & + & + & . & + & + & + & . & . \\
\hline & Galium anisophyllum & E1 & . & . & . & . & + & . & . & . & . & . & . & + & + & . & . \\
\hline & Juncus monanthos & E1 & . & . & . & . & + & + & + & . & . & . & + & . & + & + & . \\
\hline & Carex firma & E1 & . & . & + & . & . & + & . & . & . & . & . & . & . & + & + \\
\hline & Bartsia alpina & E1 & . & . & . & . & . & . & + & . & . & . & + & + & . & . & . \\
\hline & Betonica alopecuros & E1 & + & . & . & . & . & . & . & . & . & . & + & + & . & . & . \\
\hline & Carex sempervirens & E1 & . & . & . & . & + & . & . & . & . & . & + & + & . & + & . \\
\hline & Arctostaphylos alpinus & E1 & . & . & . & . & . & . & . & . & . & . & . & . & . & . & + \\
\hline
\end{tabular}




\begin{tabular}{|c|c|c|c|c|c|c|c|c|c|c|c|c|c|c|c|c|c|c|c|c|c|c|c|c|c|c|}
\hline 16 & 17 & 18 & 19 & 20 & 21 & 22 & 23 & 24 & 25 & 26 & 27 & 28 & 29 & 30 & 31 & 32 & 33 & 34 & 35 & 36 & 37 & 38 & 39 & 40 & Pr. & Fr. \\
\hline . & + & + & . & + & . & . & . & . & 1 & . & + & + & . & 1 & 1 & 1 & 1 & 1 & 1 & 1 & 1 & 1 & . & 1 & 23 & 58 \\
\hline+ & . & . & . & . & + & . & + & . & + & . & + & . & . & 1 & + & 1 & . & . & 1 & + & + & + & 1 & + & 23 & 58 \\
\hline . & . & 1 & . & 1 & + & . & . & . & . & + & 1 & + & 1 & 1 & + & 1 & 1 & + & . & . & . & + & . & 1 & 22 & 55 \\
\hline . & 1 & . & . & . & . & . & . & . & + & + & . & . & . & 2 & 1 & 1 & + & + & 1 & 1 & + & + & . & 1 & 18 & 45 \\
\hline+ & . & + & . & + & . & . & . & . & . & + & 1 & . & . & 1 & + & + & + & . & . & + & + & . & . & 4 & 14 & 35 \\
\hline . & . & . & . & + & . & + & . & . & . & . & + & . & . & . & . & + & . & . & . & . & . & + & . & + & 14 & 35 \\
\hline . & . & + & . & . & . & . & . & . & . & 1 & . & . & . & . & . & + & + & + & . & . & . & 1 & . & . & 13 & 33 \\
\hline . & . & . & . & . & . & . & . & . & . & . & . & . & . & 1 & 1 & 1 & 1 & + & 1 & + & + & . & . & + & 13 & 33 \\
\hline . & . & . & . & + & . & . & . & . & . & . & + & . & . & 1 & 1 & 1 & 1 & . & 1 & . & . & + & . & + & 13 & 33 \\
\hline . & . & + & + & + & . & . & . & . & . & . & + & . & . & . & + & . & . & . & . & . & . & . & . & . & 11 & 28 \\
\hline . & + & . & . & . & . & . & . & . & . & . & . & . & . & + & 1 & 2 & 1 & + & . & + & + & + & . & 1 & 11 & 28 \\
\hline . & . & . & . & . & . & . & . & . & . & . & . & . & . & + & + & + & . & . & + & + & + & 1 & 2 & + & 11 & 28 \\
\hline . & . & . & . & . & . & . & . & . & . & . & + & . & . & 2 & 2 & 1 & 1 & + & . & . & . & . & . & . & 9 & 23 \\
\hline . & . & . & . & . & . & . & . & + & . & . & . & . & . & + & + & + & + & + & . & . & . & . & . & . & 9 & 23 \\
\hline . & . & . & . & . & . & . & . & . & . & . & . & . & . & 1 & + & + & + & + & . & . & . & . & . & + & 7 & 18 \\
\hline . & . & . & . & . & . & . & . & . & . & . & . & . & . & 1 & + & . & + & . & + & . & . & + & . & 1 & 7 & 18 \\
\hline . & . & . & . & . & . & . & . & . & . & . & . & . & . & 1 & 1 & 1 & 1 & . & . & . & . & + & . & 1 & 7 & 18 \\
\hline . & . & . & . & . & . & . & . & . & . & . & . & . & . & . & . & . & . & . & . & . & . & + & + & . & 4 & 10 \\
\hline . & . & . & . & . & . & . & . & . & . & + & . & . & . & . & . & . & . & . & . & . & . & . & . & . & 4 & 10 \\
\hline . & . & . & . & + & . & . & . & . & . & . & . & . & . & . & . & + & . & . & . & . & . & . & . & . & 4 & 10 \\
\hline . & . & . & . & . & . & . & . & . & . & . & . & . & . & . & . & 1 & 1 & + & . & . & . & . & . & . & 3 & 8 \\
\hline . & . & $\cdot$ & . & $\cdot$ & $\mathrm{r}$ & . & . & . & . & . & + & . & . & . & . & . & . & . & . & . & . & . & . & . & 3 & 8 \\
\hline . & . & $\cdot$ & . & . & . & . & . & . & . & . & . & . & . & + & . & . & . & . & . & 1 & 1 & . & . & . & 3 & 8 \\
\hline . & . & . & . & . & . & . & . & . & . & . & . & . & . & + & + & . & + & . & . & . & . & . & . & . & 3 & 8 \\
\hline . & . & . & . & . & . & . & . & . & . & . & . & . & . & 2 & 2 & . & . & . & . & . & . & . & . & . & 3 & 8 \\
\hline . & . & . & . & . & . & . & . & . & . & . & . & . & . & . & . & . & . & . & . & . & . & . & . & + & 1 & 3 \\
\hline . & . & . & . & . & . & . & . & . & . & . & . & . & . & . & . & . & . & . & . & . & . & . & . & + & 1 & 3 \\
\hline . & . & . & . & . & . & . & . & . & . & . & . & . & . & . & . & . & . & . & . & . & . & + & . & . & 1 & 3 \\
\hline . & . & . & . & . & . & . & . & . & . & . & . & . & . & . & . & . & . & . & . & . & . & . & . & . & 1 & 3 \\
\hline . & . & . & . & . & . & . & . & . & . & . & . & . & . & . & + & . & . & . & . & . & . & . & . & . & 1 & 3 \\
\hline . & . & . & . & . & . & . & . & . & . & . & . & . & . & . & . & + & . & . & . & . & . & . & . & . & 1 & 3 \\
\hline . & . & . & . & . & . & . & . & . & . & . & . & . & . & . & . & . & . & . & . & + & . & . & . & . & 1 & 3 \\
\hline . & . & . & . & . & . & . & . & . & . & . & . & . & . & . & . & . & + & . & . & . & . & . & . & . & 1 & 3 \\
\hline . & . & . & . & . & . & . & . & . & . & . & . & . & . & . & . & . & . & . & . & . & . & . & . & + & 1 & 3 \\
\hline 1 & 1 & + & 1 & + & . & + & 1 & + & + & 1 & . & . & 3 & . & + & . & + & + & . & 1 & 1 & . & 1 & . & 31 & 78 \\
\hline+ & 1 & + & . & . & + & . & + & + & . & + & . & . & 1 & . & + & . & . & . & . & . & + & + & $\mathrm{r}$ & . & 23 & 58 \\
\hline . & 1 & + & + & + & + & . & + & . & . & + & + & . & . & . & + & + & + & + & . & . & + & . & . & . & 21 & 53 \\
\hline . & + & 1 & . & + & + & . & . & . & + & . & . & . & . & + & + & 1 & + & 1 & 1 & . & . & . & . & . & 20 & 50 \\
\hline . & . & + & + & + & . & . & . & . & . & + & . & + & + & + & . & + & + & . & . & . & . & . & . & . & 17 & 43 \\
\hline . & 1 & . & . & + & . & . & . & . & . & . & . & . & 1 & . & + & + & + & + & + & 1 & . & . & . & . & 11 & 28 \\
\hline . & . & . & + & + & + & . & + & . & . & . & . & + & . & . & + & + & + & + & . & . & . & . & 1 & . & 11 & 28 \\
\hline . & . & . & . & + & + & . & . & . & . & + & . & . & . & + & + & + & + & + & . & . & . & . & . & . & 8 & 20 \\
\hline . & . & . & . & . & . & . & . & . & . & . & . & . & . & . & . & . & . & . & . & . & + & + & . & . & 8 & 20 \\
\hline . & . & + & . & . & . & . & . & . & . & + & . & . & . & . & . & . & + & + & . & . & . & + & . & . & 8 & 20 \\
\hline . & + & . & . & . & . & . & . & . & . & + & . & . & . & . & . & . & . & . & . & . & . & . & . & . & 8 & 20 \\
\hline . & . & . & + & . & . & . & . & . & . & . & . & . & . & . & . & . & . & . & . & . & + & . & . & . & 6 & 15 \\
\hline . & . & . & $\cdot$ & $\cdot$ & . & . & . & . & . & + & . & . & + & . & . & . & . & . & . & . & . & . & . & . & 5 & 13 \\
\hline . & . & . & . & . & . & . & . & . & . & + & . & . & . & . & . & . & . & . & . & . & . & . & + & . & 5 & 13 \\
\hline . & . & . & . & . & . & . & . & . & . & + & . & . & . & . & . & . & . & . & . & . & . & . & . & . & 5 & 13 \\
\hline . & . & . & + & . & . & . & . & . & . & + & . & . & . & . & . & . & . & . & . & . & + & . & . & . & 4 & 10 \\
\hline
\end{tabular}


Number of relevé (Zaporedna številka popisa)

Helianthemum alpestre

Pedicularis rostrato-capitata

Thymus praecox subsp. polytrichus

Arabis vochinensis

Dryas octopetala

Hieracium villosum

Koeleria eriostachya

Phleum hirsutum

Phleum rhaeticum

Pulsatilla alpina subsp. austroalpina

Scabiosa lucida subsp. lucida

Hieracium pilosum

Pedicularis verticillata

Polygala alpestris

Primula wulfeniana

Ranunculus carinthiacus

Ranunculus montanus agg.

Senecio abrotanifolius

Sesleria sphaerocephala

Achillea clavenae

Carduus crassifolius

Carex mucronata

Myosotis alpestris

Oxytropis neglecta

Scabiosa lucida subsp. stricta

FB Festuco-Brometea

Bromopsis transilvanica

Carlina acaulis

Linum catharticum

Prunella grandiflora

JT Juncetea trifidi

Soldanella alpina

Anthoxanthum odoratum agg.

Gentiana pannonica

Potentilla aurea

Agrostis rupestris

LV Vaccinium gaultherioides

CU Calluno-Ulicetea

Coeloglossum viride

Hieracium lachenalii

Galium pumilum

EA Epilobietea angustifolii

Chamaenerion angustifolium

Epilobium collinum

TG Trifolio-Geranietea

Digitalis grandiflora

Vicia sylvatica

PaT Poo alpinae-Trisetalia

Campanula scheuchzeri

Poa alpina

Trollius europaeus

E1
E1
E1
E1
E1
E1
E1
E1
E1
E1
E1
E1
E1
E1
E1
E1
E1
E1
E1
E1
E1
E1
E1
E1
E1

$\begin{array}{lllllllllllllll}1 & 2 & 3 & 4 & 5 & 6 & 7 & 8 & 9 & 10 & 11 & 12 & 13 & 14 & 15\end{array}$

E1

E1

E1

E1

E1

E1

E1

E1

E1

E1

E1

E1

E1

E1

E1 r

E1

E1

E1

E1

E1

E1

$\mathrm{E} 1+++.+1++++1+++$

$\mathrm{E} 1$. . . . ++ ..+++ . +

E1 


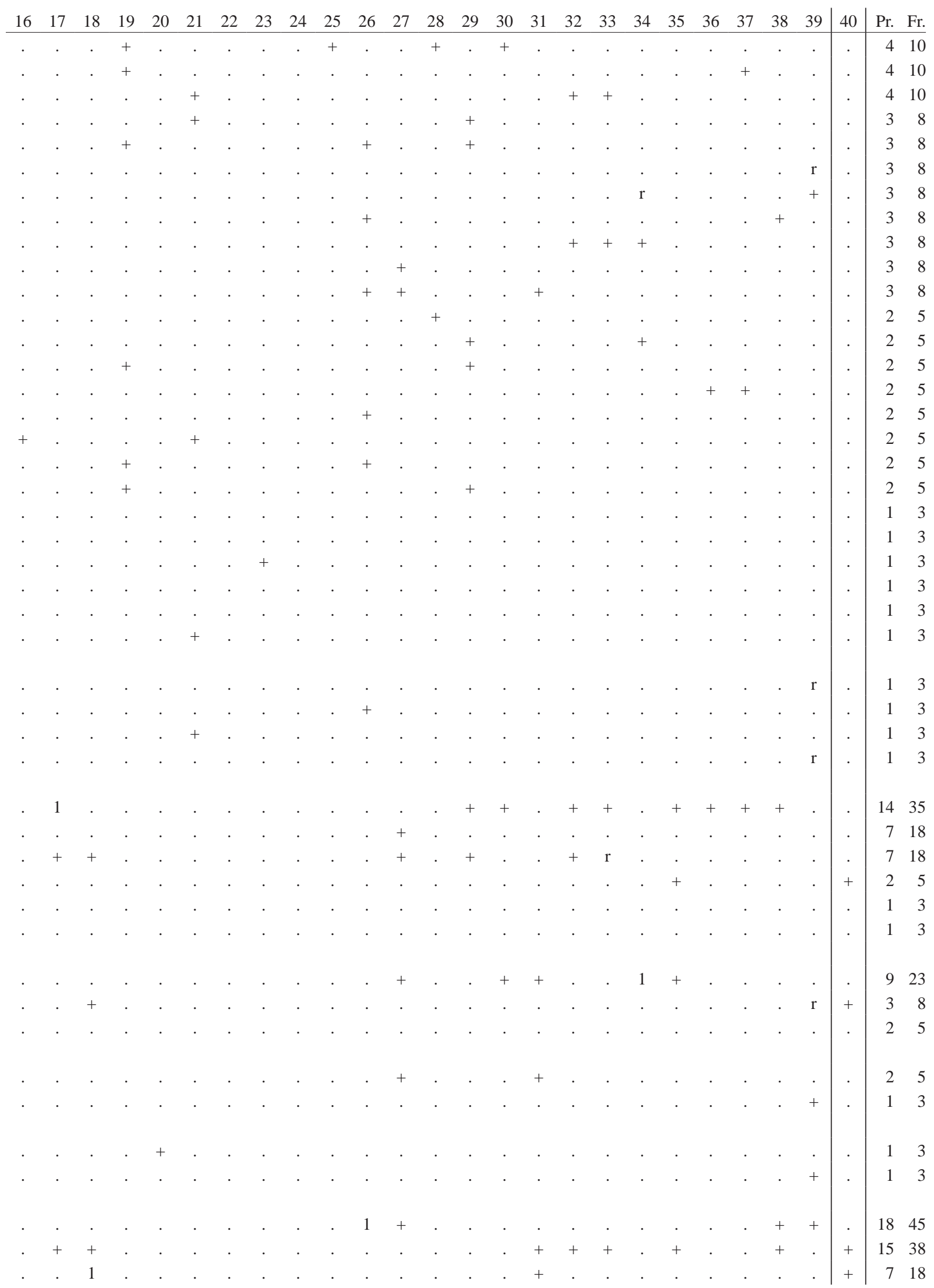




\begin{tabular}{|c|c|c|c|c|c|c|c|c|c|c|c|c|c|c|c|}
\hline Number of relevé (Zaporedna številka popisa) & & 1 & 2 & 3 & 4 & 5 & 6 & 7 & 8 & 9 & 10 & 11 & 12 & 13 & 14 \\
\hline Ranunculus nemorosus & E1 & . & - & . & . & . & . & . & + & . & . & + & $\cdot$ & . & \\
\hline Festuca nigrescens & E1 & + & . & . & . & . & . & 1 & . & . & . & . & . & . & . \\
\hline Cerastium fontanum & E1 & . & . & . & . & . & . & . & . & . & . & . & . & . & . \\
\hline Crepis aurea & E1 & . & . & . & . & . & . & . & . & . & . & . & . & . & . \\
\hline
\end{tabular}

MA Molinio-Arrhenetheretea

Deschampsia cespitosa

E1

Caltha palustris

Dactylis glomerata s.str.

Lotus corniculatus s. lat. (L. alpinus)

E1

Veronica chamaedrys

E1

Angelica sylvestris

E1

Astrantia major

Cirsium eriophorum

Galium mollugo

Leontodon hispidus

Prunella vulgaris

Taraxacum officinale

Trifolium pratense

E1

E1

E1

E1

E1

E1

E1

E1

E1

SCf Scheuchzerio-Caricetea fuscae

Selaginella selaginoides

Parnassia palustris

Pinguicula alpina

Tofieldia calyculata

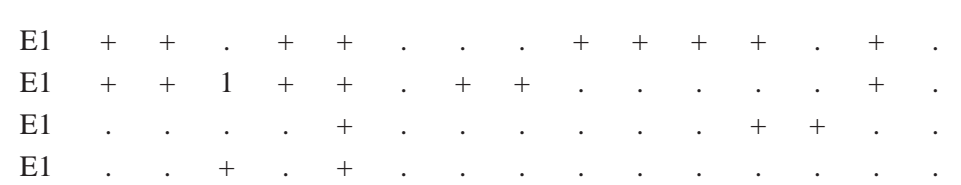

AC Arabidetalia caeruleae

Alchemilla fissa

E1

Carex capillaris

E1

Salix retusa

Carex atrata

Homogyne discolor

Saxifraga sedoides

Veronica aphylla

E1

E1

E1

E1

E1

TR Thlaspietea rotundifolii

Campanula cochleariifolia

Adenostyles glabra

Festuca nitida

Gymnocarpium robertianum

Dryopteris villarii

Cystopteris montana

E1

Trisetum argenteum

Arabis alpina

Biscutella laevigata

$\begin{array}{lllllllll}\mathrm{E} 1 & 1 & 1 & 1 & 2 & + & \cdot & + & 1 \\ \mathrm{E} 1 & + & + & + & + & + & + & + & \end{array}$

Alchemilla alpigena

E1

E1

E1

E1

E1

Minuartia austriaca

E1

Rhodiola rosea

E1

E1

Saxifraga aizoides

E1

E1

Molopospermum peloponnesiacum subsp. bauhinii E1

Campanula cespitosa

E1

Cerastium carinthiacum

E1

Festuca laxa

Geranium macrorrhizum

E1

E1

Ligusticum seguieri

E1 


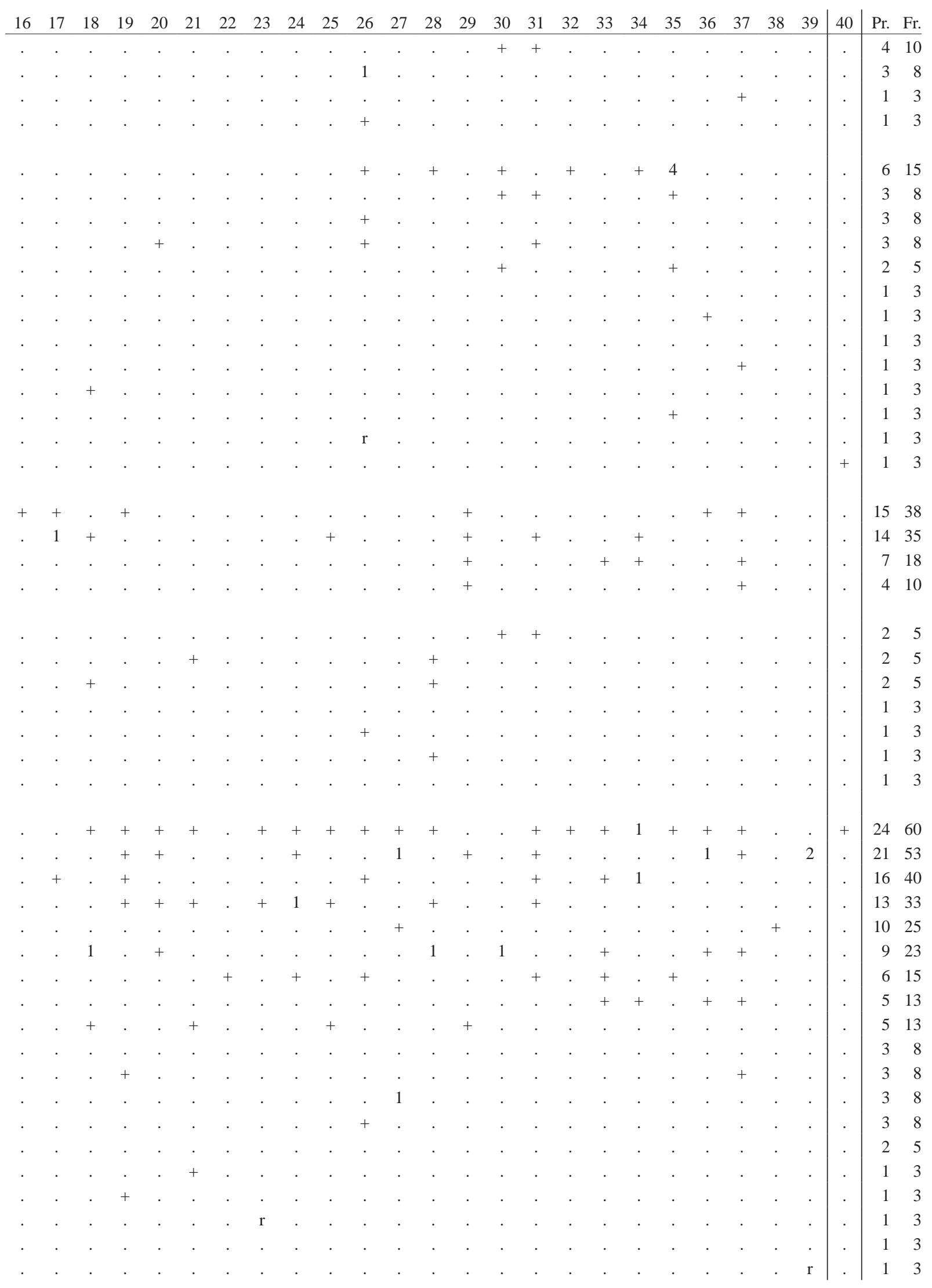




\begin{tabular}{|c|c|c|c|c|c|c|c|c|c|c|c|c|c|c|c|c|c|}
\hline & Number of relevé (Zaporedna številka popisa) & & 1 & 2 & 3 & 4 & 5 & 6 & 7 & 8 & 9 & 10 & 11 & 12 & 13 & 14 & 15 \\
\hline & Poa minor & E1 & . & . & . & . & . & . & . & . & . & . & . & . & . & . & . \\
\hline & Saxifraga caesia & E1 & . & . & . & . & . & . & . & . & . & . & . & . & . & . & . \\
\hline & Scrophularia juratensis & E1 & . & . & . & . & . & . & . & . & . & . & . & . & . & . & . \\
\hline \multirow[t]{25}{*}{ AT } & Asplenietea trichomanis & & & & & & & & & & & & & & & & \\
\hline & Asplenium viride & E1 & 1 & + & + & + & + & 1 & 1 & 1 & 1 & 1 & + & + & + & + & . \\
\hline & Cystopteris fragilis & E1 & . & + & . & + & . & + & + & 1 & + & + & + & + & + & . & . \\
\hline & Heliosperma pusillum & E1 & . & . & . & . & + & . & + & . & + & + & + & . & + & . & . \\
\hline & Moehringia muscosa & E1 & + & . & . & . & . & . & + & . & . & . & . & + & . & . & . \\
\hline & Saxifraga crustata & E1 & . & . & . & . & . & . & . & . & . & + & + & + & + & . & . \\
\hline & Polypodium vulgare & E1 & . & . & . & . & . & . & . & + & + & . & + & . & . & . & . \\
\hline & Cerastium subtriflorum & E1 & . & . & . & . & + & . & . & + & + & + & + & . & . & . & . \\
\hline & Asplenium ruta-muraria & E1 & + & . & . & . & . & + & . & . & . & . & + & + & . & . & . \\
\hline & Asplenium trichomanes & E1 & . & . & . & . & . & . & . & . & + & . & + & . & . & . & . \\
\hline & Festuca stenantha & E1 & . & . & . & . & . & . & . & + & . & . & + & . & . & . & . \\
\hline & Hieracium bifidum & E1 & . & . & . & . & . & . & . & . & . & . & . & . & . & . & . \\
\hline & Ranunculus alpestris & E1 & . & . & . & . & . & . & . & . & . & . & . & . & . & . & . \\
\hline & Soldanella minima & E1 & . & . & . & . & . & . & . & . & . & . & . & . & . & . & . \\
\hline & Cystopteris regia & E1 & . & . & . & . & + & . & . & . & . & . & . & $\mathrm{r}$ & . & . & . \\
\hline & Androsace lactea & E1 & . & . & . & . & . & . & . & . & . & . & . & . & . & . & . \\
\hline & Ranunculus traunfellneri & E1 & . & . & . & . & . & . & . & . & . & . & . & . & + & . & . \\
\hline & Bupleurum petraeum & E1 & . & . & . & + & . & . & . & . & . & . & . & . & . & . & . \\
\hline & Campanula zoysii & E1 & . & . & . & . & . & . & . & . & . & . & . & . & . & . & . \\
\hline & Festuca alpina & E1 & . & . & . & . & . & . & + & . & . & . & . & . & . & . & . \\
\hline & Hedysarum hedysaroides subsp. exaltatum & E1 & . & . & . & . & . & . & . & . & . & . & + & . & . & . & . \\
\hline & Hieracium caesium & E1 & . & . & . & . & . & . & . & . & . & . & . & . & + & . & . \\
\hline & Phyteuma sieberi & E1 & . & . & . & . & . & . & . & . & . & . & . & . & . & . & . \\
\hline & Potentilla clusiana & E1 & . & . & . & . & . & . & . & . & . & . & . & . & . & . & . \\
\hline & Valeriana elongata & E1 & . & . & . & . & . & . & . & . & . & . & . & . & . & . & . \\
\hline \multirow[t]{4}{*}{$\mathrm{O}$} & Other species (Druge vrste) & & & & & & & & & & & & & & & & \\
\hline & Alchemilla sp. & E1 & . & . & . & . & . & . & . & . & . & . & . & . & . & . & . \\
\hline & Hieracium sp. & E1 & . & . & . & . & . & . & . & + & . & . & . & . & . & . & . \\
\hline & Selaginella helvetica & E1 & . & . & . & . & . & . & . & . & . & . & . & . & . & . & . \\
\hline \multirow[t]{19}{*}{ ML } & Mosses and lichens (Mahovi in lišaji) & & & & & & & & & & & & & & & & \\
\hline & Rhytidiadelphus triquetrus & E0 & . & 1 & + & 1 & + & + & 1 & + & + & 2 & 1 & + & 2 & 1 & 3 \\
\hline & Tortella tortuosa & E0 & 1 & + & + & + & + & + & + & + & 1 & + & + & . & 1 & + & + \\
\hline & Dicranum scoparium & E0 & + & + & . & + & + & + & 1 & + & + & + & 1 & 1 & 1 & + & 1 \\
\hline & Ctenidium molluscum & E0 & + & 1 & . & + & . & + & 1 & + & 1 & . & + & 1 & . & 1 & + \\
\hline & Hylocomium splendens & E0 & . & + & + & 1 & 1 & + & + & + & 1 & 2 & 1 & 1 & 1 & + & 1 \\
\hline & Polytrichum formosum & E0 & + & + & . & . & + & 2 & + & 1 & + & + & + & + & + & + & . \\
\hline & Fissidens dubius & E0 & + & . & . & . & + & + & . & + & + & . & . & + & . & + & . \\
\hline & Peltigera leucophlebia & E0 & . & + & . & + & . & + & + & . & + & + & + & + & 1 & . & . \\
\hline & Orthothecium rufescens & E0 & + & . & . & + & + & . & . & . & . & . & + & . & + & . & . \\
\hline & Schistidium apocarpum & E0 & . & . & . & . & . & + & + & . & . & . & + & . & . & . & . \\
\hline & Plagiochila porelloides & E0 & + & . & . & . & + & + & . & . & . & + & . & . & . & . & . \\
\hline & Sphagnum sp. (inc. *S. quinquefarium) & E0 & . & 1 & + & . & . & . & . & . & . & . & . & . & . & 1 & . \\
\hline & Peltigera canina & E0 & . & . & . & . & . & . & . & + & + & + & + & . & . & . & . \\
\hline & Eurhynchium striatum & E0 & . & . & . & . & . & . & + & . & . & 1 & . & . & . & . & . \\
\hline & Plagiothecium undulatum & E0 & . & . & + & . & . & . & . & . & . & . & . & . & . & + & . \\
\hline & Bartramia halleriana & E0 & . & . & . & . & + & . & . & + & + & + & + & . & . & . & . \\
\hline & Rhytidiadelphus loreus & E0 & . & . & . & . & + & . & . & . & . & . & . & . & . & . & . \\
\hline & Cladonia pyxidata & E0 & . & . & . & . & . & . & . & . & . & . & . & . & . & . & . \\
\hline
\end{tabular}




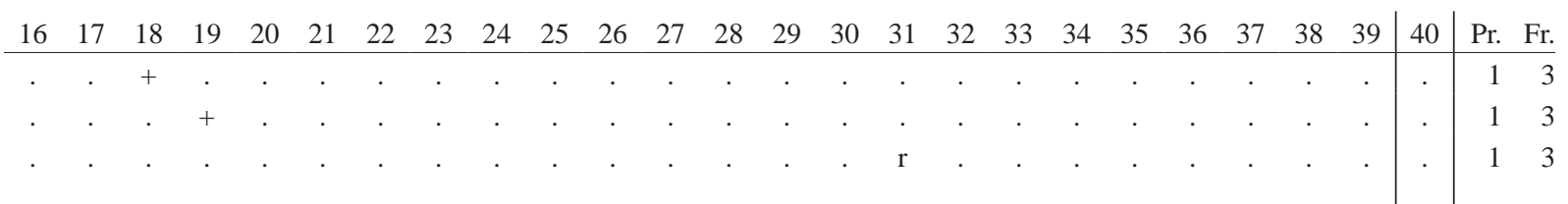

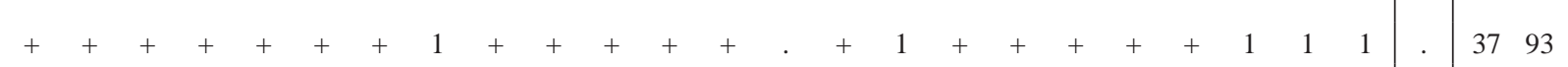

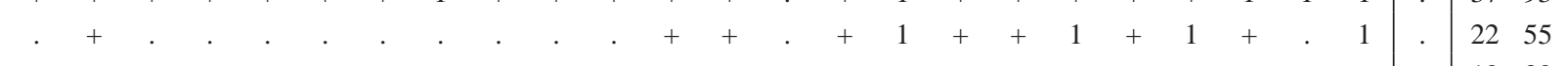

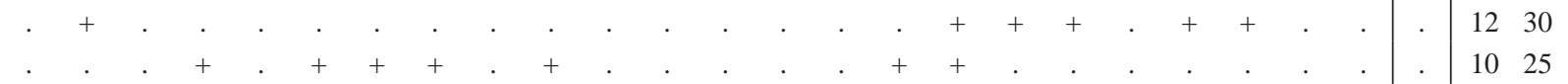

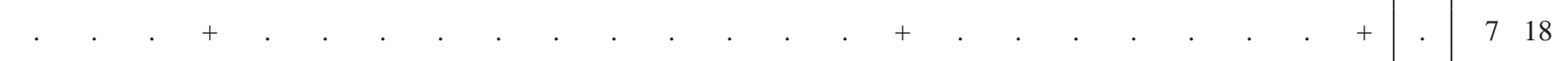

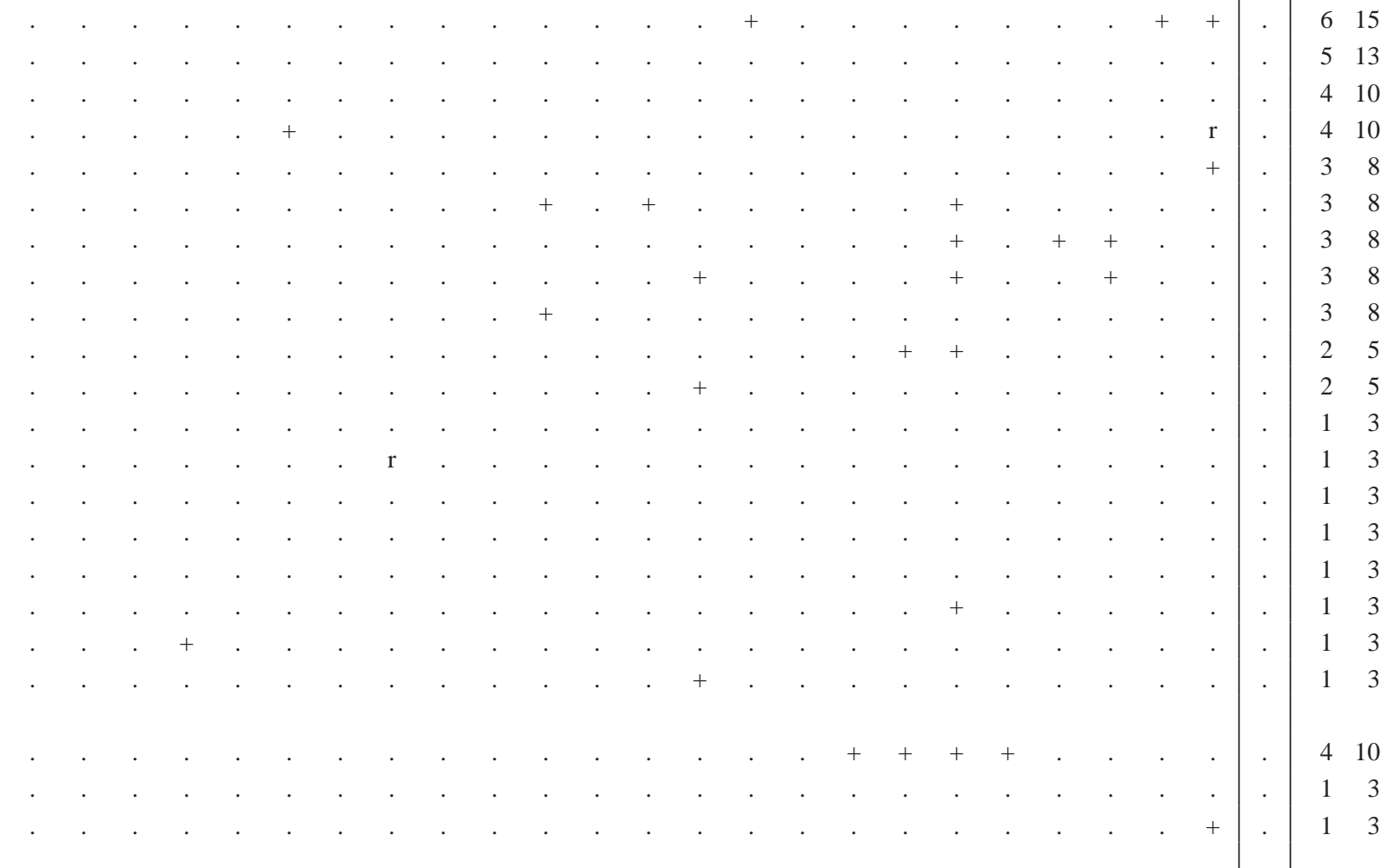

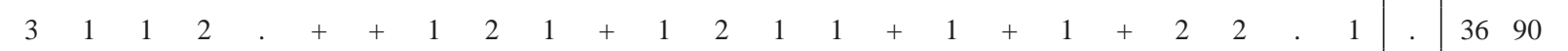

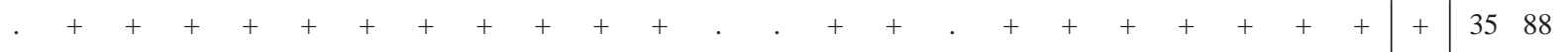

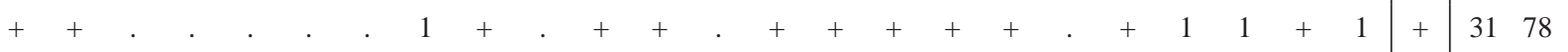

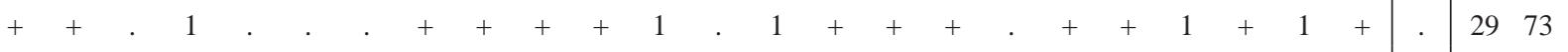

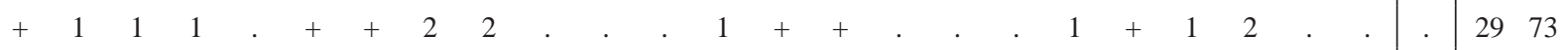

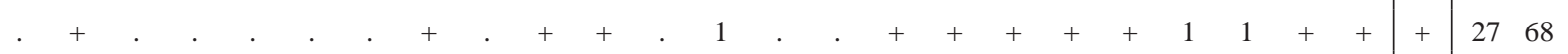

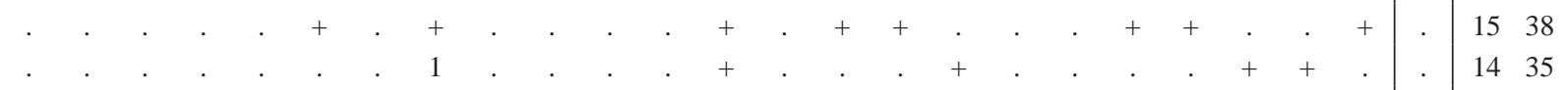

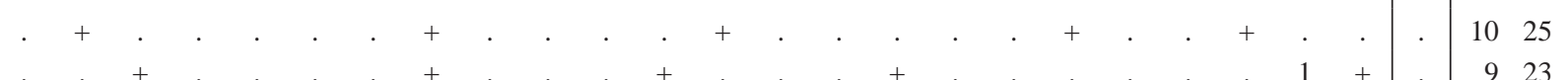

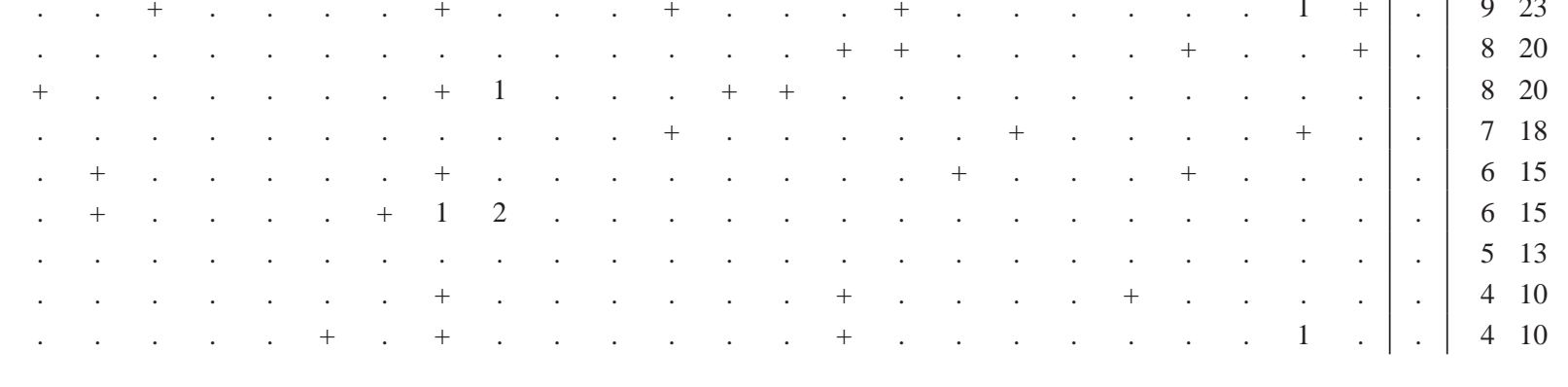




\begin{tabular}{|c|c|c|c|c|c|c|c|c|c|c|c|c|c|c|c|c|}
\hline Number of relevé (Zaporedna številka popisa) & & 1 & 2 & 3 & 4 & 5 & 6 & 7 & 8 & 9 & 10 & 11 & 12 & 13 & 14 & 15 \\
\hline Cladonia sp. & E0 & . & & . & . & . & . & . & . & + & . & + & . & . & + & . \\
\hline Conocephalum conicum & E0 & . & & . & . & . & . & . & . & . & + & + & . & + & . & . \\
\hline Mnium thomsonii & E0 & . & . & . & . & . & . & . & . & . & + & . & + & . & . & . \\
\hline Rhizomnium punctatum & E0 & . & . & . & . & . & . & + & . & . & + & + & . & . & . & . \\
\hline Cetraria islandica & E0 & . & . & . & . & . & . & . & . & . & . & . & . & . & . & . \\
\hline Letharia vulpina & E3a & . & . & . & . & . & . & . & . & . & . & . & . & . & . & . \\
\hline Isothecium alopecuroides & E0 & + & . & . & . & . & . & . & . & . & . & . & . & . & . & . \\
\hline Marchantia polymorpha & E0 & . & . & . & . & . & . & + & . & . & . & . & + & . & . & . \\
\hline Metzgeria furcata & E0 & + & . & . & . & . & . & . & . & . & . & . & . & . & . & . \\
\hline Metzgeria sp. & E0 & . & . & . & . & . & . & . & + & . & 1 & . & . & . & . & . \\
\hline Neckera crispa & E0 & . & . & . & + & . & . & . & . & . & . & + & . & . & . & . \\
\hline Plagiomnium undulatum & E0 & . & . & . & . & . & . & . & . & . & . & + & . & . & . & . \\
\hline Plagiothecium sylvaticum & E0 & . & + & . & . & . & . & + & . & . & . & . & . & . & . & . \\
\hline Polytrichum commune & E0 & . & . & . & . & . & . & . & . & . & . & . & . & . & . & . \\
\hline Musci sp. & E0 & . & . & . & + & . & . & . & . & . & . & . & . & . & . & . \\
\hline Atrichum undulatum & E0 & . & . & . & . & . & . & . & . & . & . & . & . & . & . & . \\
\hline Bryum capillare & E0 & . & . & . & . & . & . & . & . & . & . & . & + & . & . & . \\
\hline Eurhynchium sp. & E0 & . & . & . & . & . & . & . & . & . & . & + & . & . & . & . \\
\hline Homalothecium lutescens & E0 & . & . & . & . & . & . & . & . & . & . & 1 & . & . & . & . \\
\hline Hypnum cupressiforme & E0 & . & . & . & . & . & . & . & . & . & . & . & . & . & . & . \\
\hline Paraleucobryum sauteri & E0 & + & . & . & . & . & . & . & . & . & . & . & . & . & . & . \\
\hline Plagiochila asplenioides & E0 & . & . & . & . & . & . & . & . & . & . & . & + & . & . & . \\
\hline Plagiopus oederi & E0 & + & . & . & . & . & . & . & . & . & . & . & . & . & . & . \\
\hline Plagiothecium sp. & E0 & . & . & . & . & . & . & . & . & . & . & . & . & . & . & . \\
\hline Polytrichastrum formosum & E0 & . & . & . & . & . & . & . & . & . & . & . & . & . & . & 1 \\
\hline Pseudoleskeella catenulata & E0 & . & . & . & . & . & . & . & . & . & . & . & . & . & . & . \\
\hline Sanionia uncinata & E0 & . & . & . & . & . & . & . & . & . & . & . & . & . & . & . \\
\hline Solorina saccata & E0 & . & & . & + & . & . & . & . & . & . & . & . & . & . & . \\
\hline *Scapania aequiloba & E0 & . & & . & . & & . & . & . & . & . & . & . & . & . & . \\
\hline *Eurhynchium angustirete & E0 & & & $\dot{v}$ & $\cdot$ & & . &. & . & & . & . & & & . & \\
\hline
\end{tabular}

*det. Andrej Martinčič
A Limestone - apnenec
D Dolomite - dolomit
G Claystone-glinavec
L Marlstone - laporovec
Re Rendzina - rendzina
K Brown calcareous soil - rjava pokarbonatna tla
Ev Eutric brown soil - evtrična rjava tla
ID Igor Dakskobler
AR Andrej Rozman
AS Andrej Seliškar

Maximum tree diametre: 15-110 cm

Maximum tree height: $12-20 \mathrm{~m}$ 


\begin{tabular}{|c|c|c|c|c|c|c|c|c|c|c|c|c|c|c|c|c|c|c|c|c|c|c|c|c|c|c|}
\hline 16 & 17 & 18 & 19 & 20 & 21 & 22 & 23 & 24 & 25 & 26 & 27 & 28 & 29 & 30 & 31 & 32 & 33 & 34 & 35 & 36 & 37 & 38 & 39 & 40 & & Fr. \\
\hline . & + & . &. & . & . & . & . & . & . & . & . &. & . & . & & . & . &. & . & . & . & . & . &. & 4 & 10 \\
\hline · & . & . & . & . & . & . & . & . & . & . & . & . & . & . & & . & . & . & . & . & . & . & . & . & 3 & 8 \\
\hline r & . & . & . & . & . & . & . & . & . & . & . & . & . & . & & . & . & . & . & . & . & & 1 & & 3 & 8 \\
\hline · & . & . & . & . & . & . & . & . & . & . & . & . & . & . & . & . & . & . & . & . & . & . & . & . & 3 & 8 \\
\hline · & . & . & . & . & $\mathrm{r}$ & . & . & . & . & . & . & + & . & . & . & . & . & . & . & . & + & . & . & . & 3 & 8 \\
\hline . & . & . & . & . & + & + & . & . & . & . & + & . & . & . & & . & . & . & . & . & . & . & . & . & 3 & 8 \\
\hline . & . & . & . & . & . & . & . & . & . & . & . & . & . & . & . & . & . & . & . & . & . & 1 & . & . & 2 & 5 \\
\hline . & . & . & . & . & . & . & . & . & . & . & . & . & . & . & . & . & . & . & . & . & . & . & . & . & 2 & 5 \\
\hline . & . & . & . & . & . & . & . & . & . & . & . & . & . & . & . & . & . & . & . & . & . & & + & . & 2 & 5 \\
\hline · & . & . & . & . & . & . & . & . & . & . & . & . & . & . & . & . & . & . & . & . & . & . & . & . & 2 & 5 \\
\hline . & . & $\cdot$ & $\cdot$ & . & $\cdot$ & . & . & . & . & . & . & . & . & . & . & . & . & . & . & . & . & . & . & . & 2 & 5 \\
\hline . & . & . & . & . & . & . & . & . & . & . & . & . & . & . & . & . & . & . & . & . & . & . & + & . & 2 & 5 \\
\hline . & . & . & . & . & . & . & . & . & . & . & . & . & . & . & . & . & . & . & . & . & . & . & . & . & 2 & 5 \\
\hline . & . & + & . & . & . & . & . & . & . & . & . & + & . & . & . & . & . & . & . & . & . & . & . & . & 2 & 5 \\
\hline . & . & . & . & . & . & . & . & . & . & . & . & . & . & . & . & . & . & . & . & . & . & . & . & . & 1 & 3 \\
\hline . & . & . & . & . & . & . & . & . & . & . & . & . & . & . & . & . & . & . & . & . & . & . & + & . & 1 & 3 \\
\hline . & . & . & . & . & . & . & . & . & . & . & . & . & . & . & . & . & . & . & . & . & . & . & . & . & 1 & 3 \\
\hline . & . & . & . & . & . & . & . & . & . & . & . & . & . & . & . & . & . & . & . & . & . & . & . & . & 1 & 3 \\
\hline . & . & . & . & . & . & . & . & . & . & . & . & . & . & . & . & . & . & . & . & . & . & . & . & . & 1 & 3 \\
\hline . & . & . & . & . & . & . & . & . & . & . & . & . & . & . & & . & . & . & . & . & . & & + & . & 1 & 3 \\
\hline . & . & . & . & . & . & . & . & . & . & . & . & . & . & . & & . & . & . & . & . & . & . & . & . & 1 & 3 \\
\hline . & . & . & . & . & . & . & . & . & . & . & . & . & . & . & . & . & . & . & . & . & . & . & . & . & 1 & 3 \\
\hline . & . & . & . & . & . & . & . & . & . & . & . & . & . & . & . & . & . & . & . & . & . & . & . & . & 1 & 3 \\
\hline . & . & . & . & . & . & . & . & . & . & . & . & . & . & . & & . & . & . & . & . & . & . & 1 & . & 1 & 3 \\
\hline . & . & . & . & . & . & . & . & . & . & . & . & . & . & . & & . & . & . & . & . & . & . & . & . & 1 & 3 \\
\hline$\cdot$ & . & . & . & & . & . & . & . & . & . & . & . & . & . & & . & . & . & & . & & + & $\cdot$ & . & 1 & 3 \\
\hline . & . & . & . & & . & . & . & . & . & . & . & + & . & . & & . & . & . & & . & . & & . & . & 1 & 3 \\
\hline$\cdot$ & . & . & . & . & . & . & . & . & . & . & . & . & . & . & & . & . & . & . & . & . & . & . & . & 1 & 3 \\
\hline & . & . & . & & . & . & . & + & . & . & . & . & . & . & & . & . & . & $\cdot$ & . & . & & $\cdot$ & . & 1 & 3 \\
\hline & & & & & & & & + & & & & & & & & & & & & & & & & & 1 & \\
\hline
\end{tabular}


Table 10 / Tabela 10: Rhododendro hirsuti-Pinetum mugo typicum var. Alnus viridis = Rhodothamno-Pinetum mugo typicum var. Alnus viridis

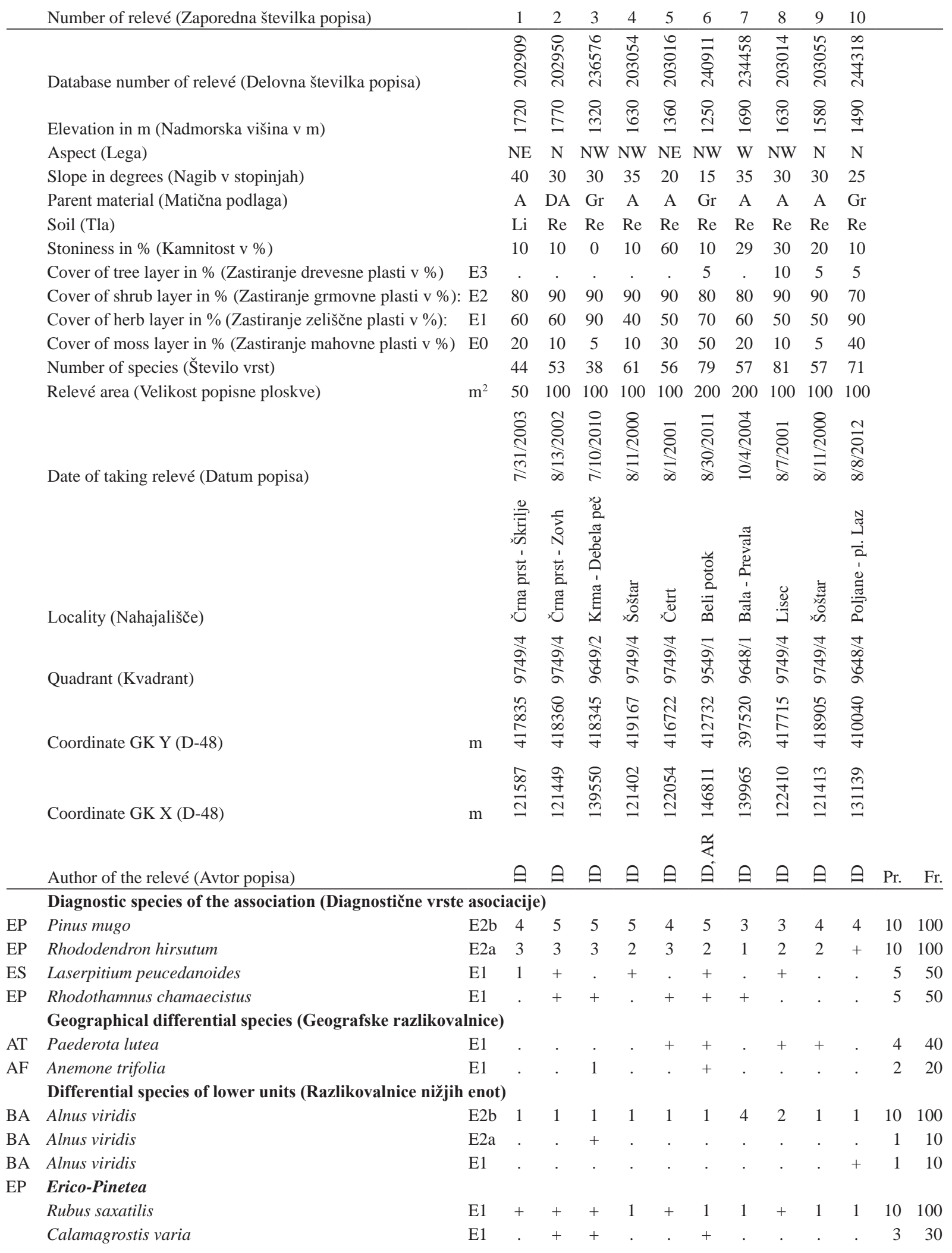


Number of relevé (Zaporedna številka popisa)

Erica carnea

Juniperus sibirica

Carex ornithopoda

Buphthalmum salicifolium

Cirsium erisithales

VP Vaccinio-Piceetea

Vaccinium myrtillus

Rosa pendulina

Lonicera caerulea

Vaccinium vitis-idaea

Clematis alpina

Valeriana tripteris

Lycopodium annotinum

Calamagrostis villosa

Luzula sylvatica

Oxalis acetosella

Solidago virgaurea

Dryopteris dilatata

Homogyne alpina

Gymnocarpium dryopteris

Huperzia selago

Maianthemum bifolium

Polystichum lonchitis

Lonicera nigra

Gentiana asclepiadea

Phegopteris connectilis

Homogyne sylvestris

Larix decidua

Larix decidua

Larix decidua

Larix decidua

Picea abies

Picea abies

Picea abies

Picea abies

Dryopteris expansa

Saxifraga cuneifolia

Luzula luzulina

Luzula luzuloides

Abies alba

Orthilia secunda

Pyrola rotundifolia

Calamagrostis arundinacea

Veronica urticifolia

Hieracium murorum

Melampyrum sylvaticum

Pyrola minor

AF Aremonio-Fagion

Cardamine enneaphyllos

Knautia drymeia

Cyclamen purpurascens

Cardamine trifolia

Helleborus niger

Rhamnus fallax

$\begin{array}{lllllllllllll} & 1 & 2 & 3 & 4 & 5 & 6 & 7 & 8 & 9 & 10 & \text { Pr. } & \text { Fr. } \\ \text { E1 } & . & . & + & . & . & 1 & + & . & . & . & 3 & 30 \\ \text { E2a } & . & . & . & + & + & \text { r } & . & . & . & . & 3 & 30 \\ \text { E1 } & . & . & . & . & . & . & + & \text { r } & . & + & 3 & 30 \\ \text { E1 } & . & . & . & + & . & . & . & . & . & . & 1 & 10 \\ \text { E1 } & . & . & . & . & . & . & + & . & . & . & 1 & 10\end{array}$

$\begin{array}{llllllllllllll}\mathrm{E} 1 & 2 & 3 & 3 & 1 & 2 & 2 & 1 & + & + & 9 & 90\end{array}$

$\mathrm{E} 2 \mathrm{a} 1+11+++11.90$

$\mathrm{E} 2 \mathrm{a}+++++++1.90$

$\begin{array}{llllllllllll}\mathrm{E} 1 & 2 & 2 & 2 & 2 & & 2 & 8\end{array}$

$\mathrm{E} 2 \mathrm{a} 1+++11++.+88$

$\mathrm{E} 1++.++++1$. $1+80$

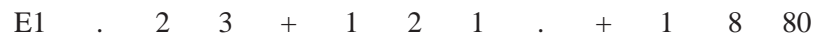

$\mathrm{E} 1+1++1.2 .+70$

$\mathrm{E} 1 \cdot+++1+\ldots+470$

$\mathrm{E} 1 .+1 .+1+++470$

$\mathrm{E} 1 .+++.+1++470$

$\mathrm{E} 1 .++++\ldots+1+70$

$\mathrm{E} 1+1+1.1 .+2 .+60$

$\mathrm{E} 1++.2 .1+1+.660$

$\mathrm{E} 1+++.+.+. \quad . \quad 1 \quad 6 \quad 60$

$\mathrm{E} 1+.++.+2.550$

$\mathrm{E} 1 .+2 .+\mathrm{t}+\mathrm{r} .50$

$\mathrm{E} 2 \mathrm{a} .+2 .+1+4.440$

$\mathrm{E} 1$. . . . $1.4 .+4.40$

$\mathrm{E} 1+. \quad . \quad 1 \quad 2 . \quad . \quad 530$

$\mathrm{E} 1+$. . . 1 . 1.930

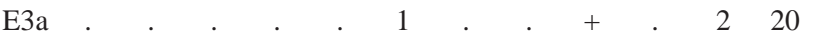

$\mathrm{E} 2 \mathrm{~b} .+\mathrm{r} .+.+2.30$

$\mathrm{E} 2 \mathrm{a} . .+. \quad . \quad . \quad . \quad . \quad . \quad 1010$

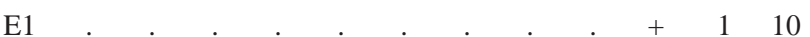

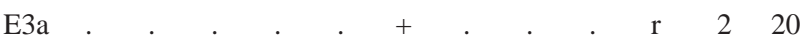

$\mathrm{E} 2 \mathrm{~b} .2 . \mathrm{r}+\mathrm{r} . \quad . \quad 330$

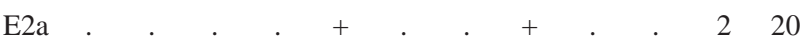

$\mathrm{E} 1$. . . . . . . +20

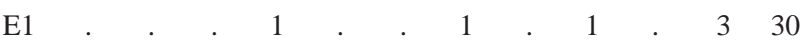

$\mathrm{E} 1$. . . . . . . . 2020

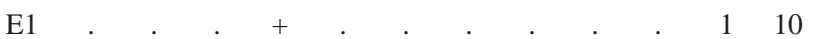

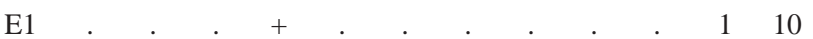

$\mathrm{E} 2$. . . . . . . . . . . 110

$\mathrm{E} 1$. . . . . + . . . . 110

$\mathrm{E} 1$. . . . . $\quad+\quad . \quad . \quad . \quad . \quad 110$

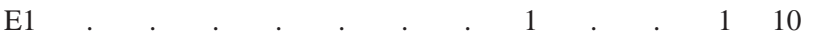

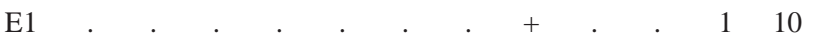

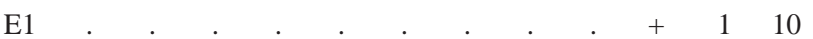

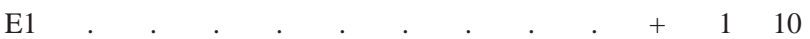

$\mathrm{E} 1 . .5 . \quad . \quad . \quad . \quad 10$

$\mathrm{E} 1 .+1 .+1+\ldots+60$

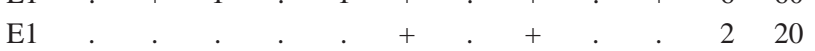

$\mathrm{E} 1$. . . . . . . . . 110

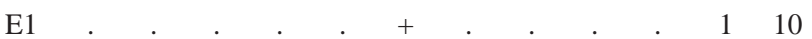

$\mathrm{E} 1$. . . . . $\quad+\quad$. . . . 110

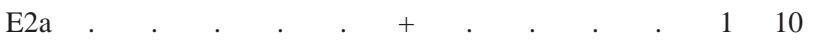


Number of relevé (Zaporedna številka popisa)

$\begin{array}{llllllllllll}1 & 2 & 3 & 4 & 5 & 6 & 7 & 8 & 9 & 10 & \text { Pr. } & \text { Fr. }\end{array}$

FS Fagetalia sylvaticae

Prenanthes purpurea

Galeobdolon flavidum

Melica nutans

Paris quadrifolia

Lilium martagon

Epilobium montanum

Daphne mezereum

Mercurialis perennis

Chrysosplenium alternifolium

Acer pseudoplatanus

Dryopteris filix-mas

Galium laevigatum

Symphytum tuberosum

Fagus sylvatica

Lonicera alpigena

Cypripedium calceolus

Adoxa moschatellina

Actaea spicata

Scrophularia nodosa

Polystichum aculeatum

QF Querco-Fagetea

Anemone nemorosa

Hepatica nobilis

Convallaria majalis

Sorbus aria

Sorbus aria

BA Betulo carpaticae-Alnetea viridis

Salix appendiculata

Salix appendiculata

Salix glabra

Salix waldsteiniana

Sorbus chamaemespilus

SSC Sambuco-Salicion capreae

Sorbus aucuparia

Sorbus aucuparia

Sorbus aucuparia

Rubus idaeus

Hypericum hirsutum

MuA Mulgedio-Aconitetea

Viola biflora

Veratrum album

Geranium sylvaticum

Polygonatum verticillatum

Ranunculus platanifolius

Athyrium filix-femina

Adenostyles alliariae

Hypericum maculatum

Saxifraga rotundifolia

Senecio cacaliaster

Carduus personata

Chaerophyllum villarsii

Cicerbita alpina

Doronicum austriacum

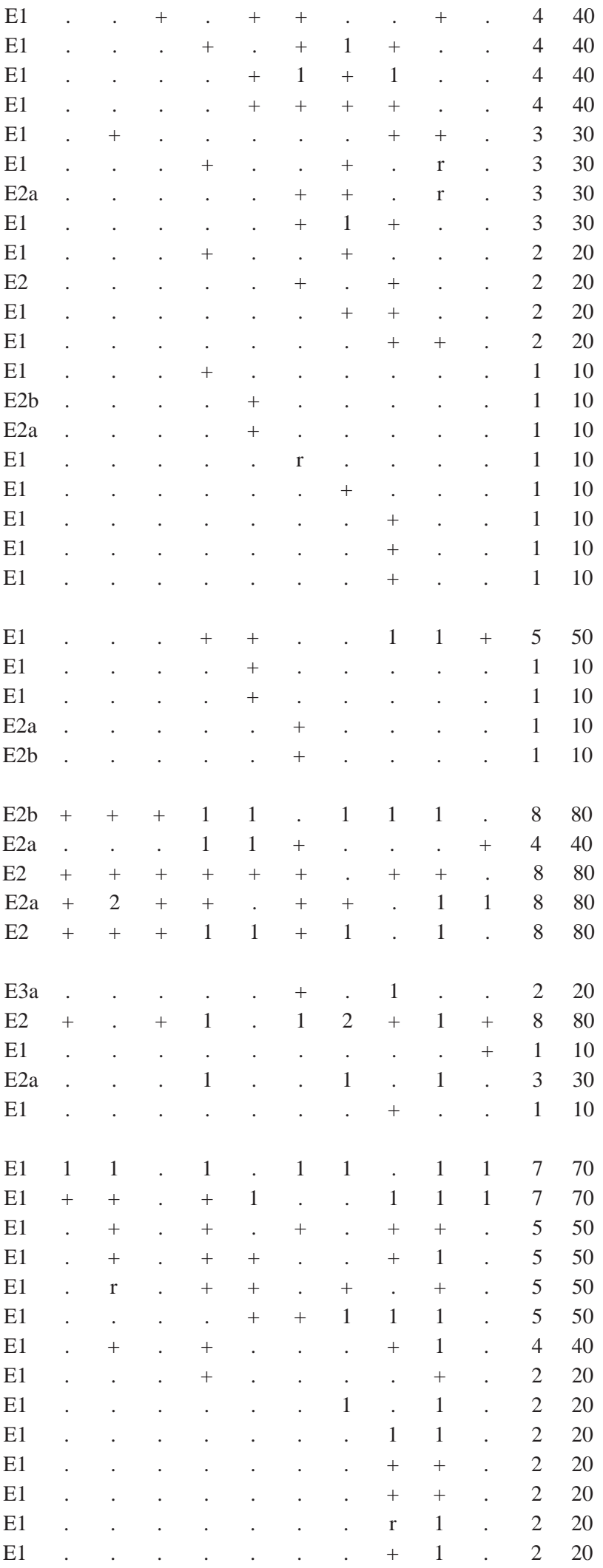


Number of relevé (Zaporedna številka popisa)

Heracleum sphondylium subsp. montanum

Aconitum lycoctonum subsp. ranunculifolium

Allium victorialis

Phyteuma ovatum

Crepis pyrenaica

Eryngium alpinum

Lathyrus occidentalis var. montanus

Myrrhis odorata

Poa hybrida

Thalictrum aquilegiifolium

Stellaria nemorum

Crepis paludosa

Geum rivale

Epilobium alpestre

ES Elyno-Seslerietea

Sesleria caerulea subsp. calcaria

Astrantia bavarica

Carex ferruginea

Campanula witasekiana

Heliosperma alpestre

Aster bellidiastrum

Juncus monanthos

Carduus crassifolius

Koeleria eriostachya

Anemone narcissiflora

Hieracium villosum

Linum julicum

Helianthemum alpestre

Festuca calva

Helianthemum nummularium subsp. grandiflorum

Betonica alopecuros

Pulsatilla alpina

Serratula tinctoria subsp. macrocephala

Agrostis alpina

Bartsia alpina

Carex firma

Carex sempervirens

Dryas octopetala

Polygonum viviparum

Salix alpina

JT Juncetea trifidi

Soldanella alpina

Anthoxanthum odoratum agg.

Agrostis rupestris

Gentiana pannonica

Potentilla aurea

LV Loiseleurio-Vaccinietea

Empetrum hermaphroditum

Arctostaphylos alpinus

Vaccinium gaultherioides

SCf Scheuchzerio-Caricetea fuscae

Parnassia palustris

Pinguicula alpina

Selaginella selaginoides

$\begin{array}{lll} & 1 & 2 \\ \text { E1 } & \cdot & \cdot \\ \text { E1 } & \cdot & \cdot \\ \text { E1 } & \cdot & + \\ \text { E1 } & \cdot & + \\ \text { E1 } & \cdot & \cdot \\ \text { E1 } & \cdot & \cdot \\ \text { E1 } & \cdot & \cdot \\ \text { E1 } & \cdot & \cdot \\ \text { E1 } & \cdot & \cdot \\ \text { E1 } & \cdot & \cdot \\ \text { E1 } & \cdot & \cdot \\ \text { E1 } & \cdot & \cdot \\ \text { E1 } & \cdot & \cdot \\ \text { E1 } & \cdot & \cdot\end{array}$

$\begin{array}{lllllllll}4 & 5 & 6 & 7 & 8 & 9 & 10 & \text { Pr. } & \text { Fr. }\end{array}$

$\mathrm{E} 11+. \quad 1.5 . \quad+\quad . \quad \begin{array}{llll}1 & 5 & 50\end{array}$

$\mathrm{E} 1++.+$. $+\mathrm{r} .+550$

$\mathrm{E} 1 .+.+2 .+2 .+30$

$\mathrm{E} 1$...$++2 .+330$

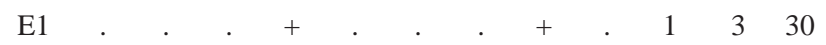

$\mathrm{E} 1 .+.+2+.+30$

$\mathrm{E} 1 .+. .5 .+2 .+20$

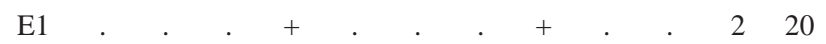

$\mathrm{E} 1 .+. .+. \quad . \quad+20$

$\mathrm{E} 1+$. . . . . . . . . 110

$\mathrm{E} 1+$. . . . . . . . . . 110

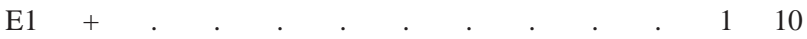

$\mathrm{E} 1$. . + . . . . . . . . 1010

$\mathrm{E} 1$. $\quad . \quad . \quad . \quad . \quad . \quad+\quad . \quad . \quad . \quad 110$

$\mathrm{E} 1$. . . . . . . $\quad+\quad$.

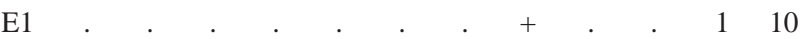

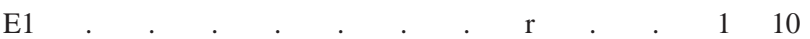

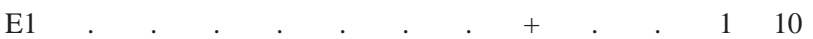

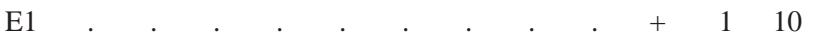

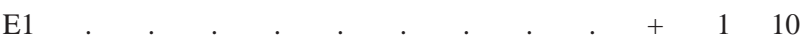

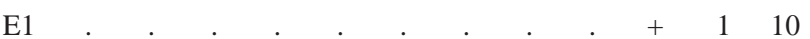

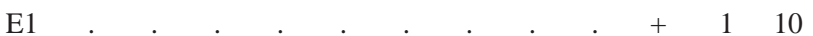

$\mathrm{E} 1.5 .5 .5 .+10$

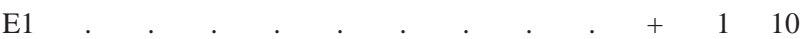

$\mathrm{E} 1$. $. \quad . \quad . \quad . \quad . \quad . \quad . \quad . \quad+\quad 110$

$\mathrm{E} 1 .+.+. \quad . \quad+\quad+20$

$\mathrm{E} 1$. . . . . . . . . 220

$\mathrm{E} 1$. $. \quad . \quad . \quad . \quad . \quad . \quad . \quad . \quad+\quad 110$

$\mathrm{E} 1.5 .5 . \quad . \quad . \quad . \quad . \quad 110$

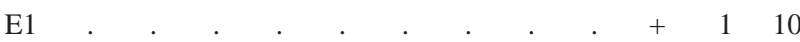

\begin{tabular}{llllllllll|l|ll}
$\mathrm{E} 1$ & + &. & + &. &. &. &. &. & $\cdot$ & 2 & 3 & 30 \\
$\mathrm{E} 1$ & $\cdot$ & + & $\cdot$ & $\cdot$ & $\cdot$ & $\cdot$ & $\cdot$ & $\cdot$ & $\cdot$ & 2 & 2 & 20 \\
$\mathrm{E} 1$ &. &. &. &. &. &. &. &. &. & 3 & 1 & 10
\end{tabular}

$\mathrm{E} 1++.+2 . \mathrm{r}++660$

$\mathrm{E} 1+.+.+.+2 .+30$

$\mathrm{E} 1+.+. \quad+\quad . \quad . \quad . \quad \begin{array}{lllll} & 1 & 3 & 30\end{array}$ 
Number of relevé (Zaporedna številka popisa)

Tofieldia calyculata

CU Calluno-Ulicetea

Potentilla erecta

Hieracium lachenalii

PaT Poo alpinae-Trisetalia

Campanula scheuchzeri

Poa alpina

Trollius europaeus

Festuca nigrescens

Cerastium fontanum

MA Molinio-Arrhenatheretea

Galium album

Deschampsia cespitosa

Angelica sylvestris

Dactylis glomerata s.str.

AC Arabidetalia caeruleae

Salix retusa

Carex atrata

Carex capillaris

Homogyne discolor

TR Thlaspietea rotundifolii

Festuca nitida

Adenostyles glabra

Gymnocarpium robertianum

Dryopteris villarii

Astrantia carniolica

Cystopteris montana

Rhodiola rosea

AT Asplenietea trichomanis

Asplenium viride

Valeriana saxatilis

Cystopteris fragilis

Saxifraga crustata

Campanula zoysii

Heliosperma pusillum

Moehringia muscosa

O Other species (Druge vrste)

Cirsium sp.

Carduus sp.

Alchemilla sp.

ML Mosses and lichens (Mahovi in lišaji)

Rhytidiadelphus triquetrus

Tortella tortuosa

Hylocomium splendens

Dicranum scoparium

Orthothecium rufescens

Polytrichum formosum

Rhytidiadelphus loreus

Sphagnum sp.

Ctenidium molluscum

Rhizomnium punctatum

Peltigera leucophlebia

Schistidium apocarpum

Dicranum sp.

$\begin{array}{rrrrrrrrrrrrr} & 1 & 2 & 3 & 4 & 5 & 6 & 7 & 8 & 9 & 10 & \text { Pr. } & \text { Fr. } \\ \mathrm{E} 1 & + & . & . & . & . & . & . & . & . & . & 1 & 10\end{array}$

E1

E1

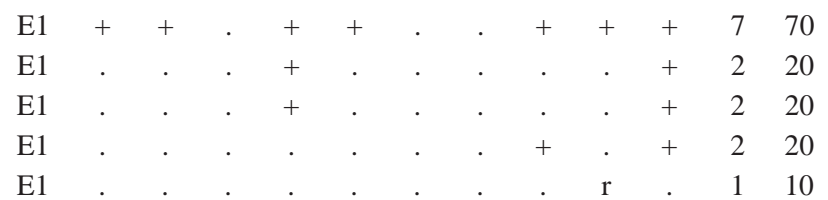

$\mathrm{E} 1 .+. \quad+\quad . \quad . \quad . \quad . \quad . \quad 110$

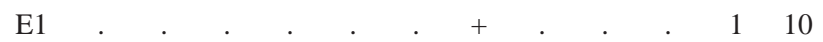

$\mathrm{E} 1 . .5 . \quad . \quad . \quad . \quad . \quad 110$

$\mathrm{E} 1 . \quad . \quad . \quad . \quad . \quad+\quad . \quad . \quad 110$

E1 . . . . . . . . . . .

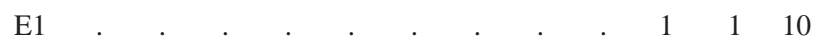

$\mathrm{E} 1$. . . . . . . . . . . $10 \begin{array}{llll}1 & 1 & 1\end{array}$

$\mathrm{E} 1 . .5 . \quad . \quad . \quad . \quad+\quad 110$

$\mathrm{E} 1$. . . $+.+4+50$

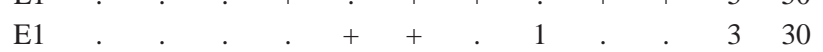

$\mathrm{E} 1$. . . . . . . . 2 . 20

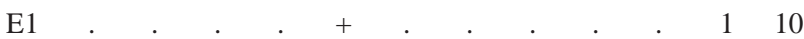

$\mathrm{E} 1$. . . . . $\quad+\quad . \quad . \quad . \quad . \quad 110$

$\mathrm{E} 1$. . . . . $\quad+\quad . \quad . \quad . \quad . \quad 1010$

$\mathrm{E} 1$. $\quad . \quad . \quad . \quad . \quad . \quad . \quad . \quad+\quad . \quad 110$

$\mathrm{E} 1 .+.+1+1+.+660$

$\mathrm{E} 1++.+2 .+. \quad$. $\quad$. 30

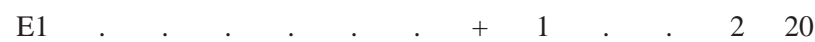

$\mathrm{E} 1+$. . . . . . . . . 110

$\mathrm{E} 1 \ldots \mathrm{r} .-10$

$\mathrm{E} 1$. . . . . + . . . . 110

$\mathrm{E} 1 . . \quad . \quad . \quad+. \quad . \quad . \quad 1010$

$\mathrm{E} 1$. . . . . $\quad+\quad$. . . . . 1010

$\mathrm{E} 1 . .5 . \quad . \quad . \quad . \quad . \quad . \quad 110$

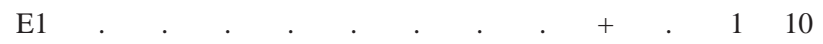

$\mathrm{E} 0$. $1+\begin{array}{lllllllll} & 1 & 1 & 2 & + & 1 & 8 & 80\end{array}$

$\mathrm{E} 0+++.+1 .+1+70$

$\mathrm{E} 0+.1+2+. \quad 2.460$

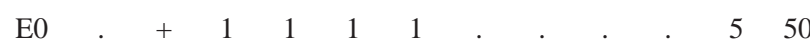

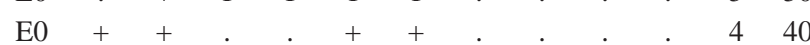

$\mathrm{E} 0+$. . $1+1$. . . 440

$\mathrm{E} 0 .+.+.+.+.4440$

$\mathrm{E} 0+. \quad 1.5 . \quad 2 . \quad . \quad 3 \quad 30$

$\mathrm{E} 0$. . . . +1.30

$\mathrm{E} 0$. + . . . . . . . 2020

$\mathrm{E} 0$. ...$++. \quad . \quad 2 \quad 20$

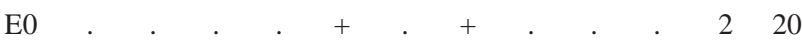

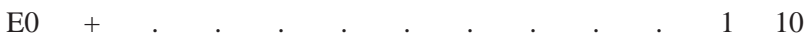


Number of relevé (Zaporedna številka popisa)

Mnium thomsonii

Bazzania trilobata

Fissidens dubius

Plagiochila asplenioides

Plagiothecium undulatum

Cladonia sp.

Polytrichum commune

Sanionia uncinata

Cetraria islandica

Musci sp.

\begin{tabular}{|c|c|c|c|c|c|c|c|c|c|c|c|}
\hline 1 & 2 & 3 & 4 & 5 & 6 & 7 & 8 & 9 & 10 & Pr. & Fr. \\
\hline E0 & + & . & . & . & . & & . & . & . & 1 & 10 \\
\hline E0 & . & . & . & + & . & $\cdot$ & . & . & . & 1 & 10 \\
\hline E0 & . & . & . & + & . & $\cdot$ & . & . & . & 1 & 10 \\
\hline E0 & . & . & . & . & + & · & . & . & . & 1 & 0 \\
\hline E0 & . & . & . & . & + & • & . & . & . & 1 & 10 \\
\hline E0 & . & . & . & . & - & . & + & . & . & 1 & 10 \\
\hline E0 & . & . & . & - & - & • & . & . & 1 & 1 & 10 \\
\hline E0 & . & . & . & - & - & - & . & • & 1 & 1 & \\
\hline E0 & . & . & . & . & . & - & . & . & 1 & 1 & 10 \\
\hline E0 & . & . & . & . & . & . & . & . & + & 1 & \\
\hline
\end{tabular}
A Limestone - apnenec
D Dolomite-dolomit
G Claystone - glinavec
Gr Gravel - pobočni grušč
Re Rendzina - rendzina
Li Lithosol-kamnišče
ID Igor Dakskobler
AR Andrej Rozman 